Imperfektionsempfindlichkeit und

Grenzlasten von Schalentragwerken

Jens Pontow

Institut für Statik

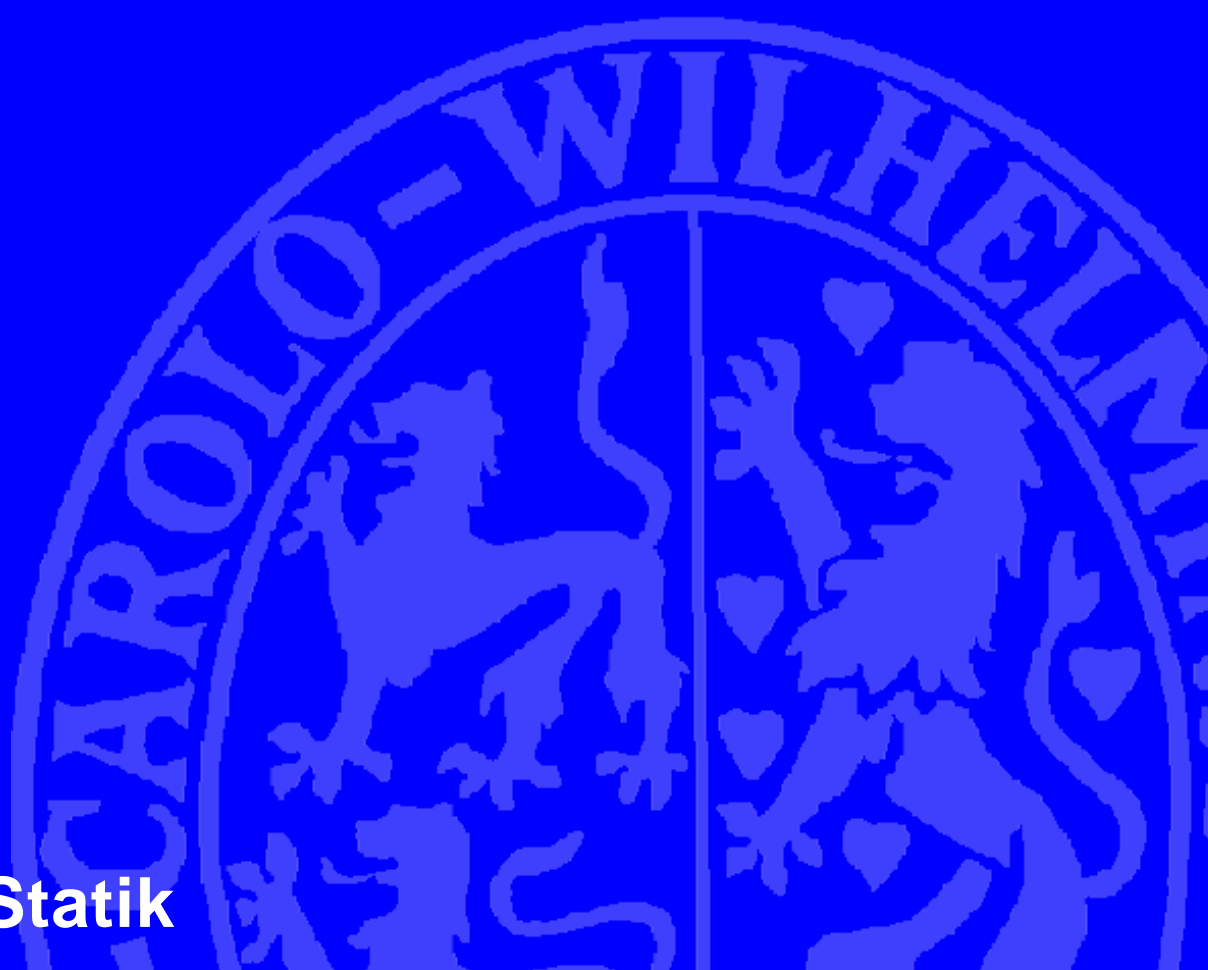

Technische Universität Braunschweig

Bericht Nr. 2009-107 


\title{
Imperfektionsempfindlichkeit und Grenzlasten von Schalentragwerken
}

\author{
Von der Fakultät \\ Architektur, Bauingenieurwesen und Umweltwissenschaften \\ der Technischen Universität Carolo-Wilhelmina zu Braunschweig \\ zur Erlangung des Grades eines Doktor-Ingenieurs (Dr.-Ing.) \\ genehmigte Dissertation
}

von

Dipl.-Ing. Jens Pontow aus Crivitz

Eingereicht am

Mündliche Prüfung am

Berichter

Braunschweig 2009
21. Dezember 2007

22. September 2008

Prof. Dr.-Ing. Dieter Dinkler

Prof. Dr.-Ing. Peter Horst

Prof. Dr.-Ing. Werner Schneider 
ISBN 978-3-926031-04-4

Herausgeber: Prof. Dr.-Ing. Dieter Dinkler

(C) Institut für Statik, Technische Universität Braunschweig, 2009 


\title{
Kurzfassung
}

Die Imperfektionsempfindlichkeit und die Grenzlasten von Schalentragwerken sind intensiv diskutierte Phänomene. Mit dem Störenergie-Konzept ist die Imperfektionsempfindlichkeit mittels einer energetischen Größe, der Störenergie, zu bewerten. Die Störenergie ermöglicht auch die Bewertung der statischen Grenzlasten verschiedener Beulfälle und einen Vergleich der Regelwerke zum Beulsicherheitsnachweis. Kinetische Grenzlasten sind mit dem Stabilitätsgrad ermittelbar. Ferner ist das StörenergieKonzept Grundlage der lastniveauspezifischen Modifikation der Imperfektionsempfindlichkeit. Die Untersuchungen zeigen die Bedeutung des Störenergie-Konzeptes sowohl für die Ermittlung ungünstiger Störlasten als auch für die Beschreibung der Widerstandsfähigkeit von Schalentragwerken gegenüber Störungen auf.

\begin{abstract}
The imperfection sensitivity and the limit loads of shell structures are widely discussed phenomena. The perturbation energy concept enables the assessment of the imperfection sensitivity by one energy value, namely the perturbation energy. The perturbation energy also allows to evaluate the static limit loads of different buckling cases and to compare the design rules relevant for the verification of stability. Kinetic limit loads may be determined by the degree of stability. Furthermore, the perturbation energy concept provides the basis for the load-level-specific modification of the imperfection sensitivity. The investigations highlight the importance of the perturbation energy concept not only for the determination of unfavourable perturbation loads but also for the evaluation of the buckling resistance of shell structures.
\end{abstract}





\section{Inhalt}

1. Einleitung 1

1.1 Stand des Wissens . . . . . . . . . . . . . . . . . . . . 1

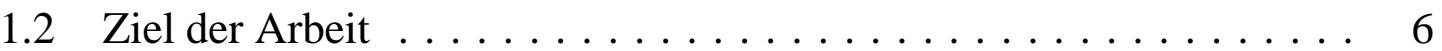

2. Berechnungsmodell $\quad 7$

2.1 Kontinuumsmechanische Grundlagen . . . . . . . . . . . . . 7

2.2 Schalentheorien . . . . . . . . . . . . . . . . 10

2.3 Integrales Plastizitätsmodell . . . . . . . . . . . . . . . . . . . . . 14

2.4 Faserverbundwerkstoffe . . . . . . . . . . . . . . . . . . . . . 19

2.5 Lösung diskretisierter Anfangs-Randwertaufgaben . . . . . . . . . . 22

2.5.1 Inkrementelle Formulierung . . . . . . . . . . . . . . . 22

2.5.2 Inhomogene Gleichungssysteme . . . . . . . . . . . 23

2.5.3 Eigenwertprobleme .................. 25

2.5.4 Integration der Bewegungsgleichung . . . . . . . . . . 26

2.5.5 Funktionalanalytische Betrachtungen . . . . . . . . . . 27

2.5.6 Versteifungseffekte .................... 30

3. Stabilität von Systemen 33

3.1 Bewegungen und Attraktoren . . . . . . . . . . . . . 33

3.2 Stabilitätsdefinitionen . . . . . . . . . . . . . . . . 34

3.3 Lösungsmethoden . . . . . . . . . . . . . . . . 36

3.4 Stabilitätskriterien . . . . . . . . . . . . . . . . . 36

3.5 Singuläre Punkte . . . . . . . . . . . . . . . . . . 41

3.6 Imperfektionsempfindlichkeit $\ldots \ldots \ldots \ldots \ldots \ldots$

4. Störenergie-Konzept 45

4.1 Einführung . . . . . . . . . . . . . . . . . . 45

4.2 Identifikation kritischer Zustände . . . . . . . . . . . . . . 46

4.2.1 Modell bei elastischem Materialverhalten . . . . . . . . . 46

4.2.2 Modell bei elastisch-plastischem Materialverhalten . . . . . . 50

4.3 Grundenergie, Störenergie und Grenzlasten . . . . . . . . . . . . . 52

4.3.1 Grundenergie und Störenergie . . . . . . . . . . . . . . 52

4.3.2 Normierung der Störenergie . . . . . . . . . . . . . . . . . . 54

4.3.3 Statische und kinetische Grenzlasten . . . . . . . . . . . . . 54

4.3 .4 Einflussfaktoren . . . . . . . . . . . . . . . 55

4.4 Berücksichtigung weiterer nicht anfänglicher Störungen . . . . . . . 59 
4.5 Bewertung des Einflusses anfänglicher Störungen $\ldots \ldots \ldots \ldots$. . . 60

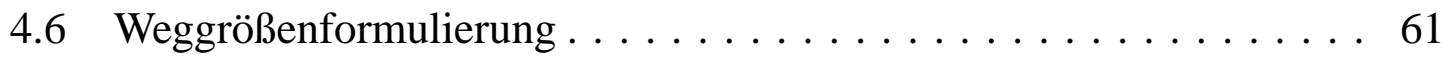

4.6.1 Identifikation kritischer Zustände und Störenergie . . . . . . . 61

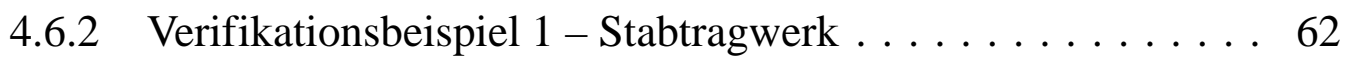

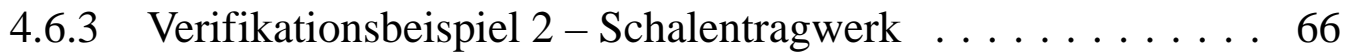

4.7 Stabilitätstheoretische Klassifizierung $\ldots \ldots \ldots \ldots \ldots \ldots 67$

5. Statische Grenzlasten von Kreiszylinderschalen 69

5.1 Konstante Axiallast . . . . . . . . . . . . . . . . . . . . . . . . . 69

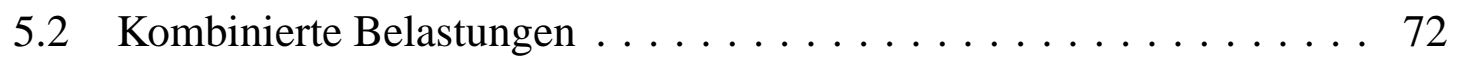

5.3 Einfluss von Störungen, Temperatur und Aussteifungen . . . . . . 74

5.4 Kreiszylinderschalen aus Faserverbundwerkstoff . . . . . . . . . . . 79

6. Statische Grenzlasten von Kugelschalen 85

6.1 Konstante Radialdruckbelastung . . . . . . . . . . . . . . . 85

6.1 .1 Eingespannte Kugelschalen $\ldots \ldots \ldots \ldots \ldots \ldots$. . . . 86

6.1.2 Unverschieblich gelenkig gelagerte Kugelschalen . . . . . . 89

6.1.3 Radial verschieblich gelagerte Kugelschalen . . . . . . . . 91

6.1.4 Kugelschalensegmente . . . . . . . . . . . . . . 92

6.1.5 Vollkugelschalen ....................... 94

6.2 Veränderliche Radialdruckbelastung . . . . . . . . . . . . . . 95

6.3 Bewertung des Tragverhaltens von Kugel- und Kreiszylinderschalen . 96

7. Statische Grenzlasten ausgewählter Schalenbeulfälle 99

7.1 Kegelschalen unter Meridianlast . . . . . . . . . . . . . . . . 99

7.2 Rotationsparaboloide unter verschiedenen Belastungen . . . . . . . . 102

7.3 Zusammengesetzte Schalen unter Radialdruck . . . . . . . . . . . . 103

8. Kinetische Grenzlasten und Optimierung von Schalentragwerken 105

8.1 Kugelschale unter Radialdruck und Impulsbelastung . . . . . . . . . . 105

8.1.1 Genauigkeit des Stabilitätsgrades . . . . . . . . . . . . . . 106

8.1.2 Räumliche und zeitliche Verteilung der Störlast . . . . . . . . 108

8.1.3 Analyse eines Beulvorganges . . . . . . . . . . . . . 113

8.2 Kreiszylinderschale unter Axiallast und Impulsbelastung . . . . . . . . 115

8.3 Zur Modifikation der Imperfektionsempfindlichkeit . . . . . . . . . 118

9. Zusammenfassung und Ausblick 121

$\begin{array}{ll}\text { Summary and Outlook } & 123\end{array}$

$\begin{array}{ll}\text { Literatur } & 125\end{array}$ 


\section{Nomenklatur}

Indizes bzgl. der Koordinaten repräsentieren Laufvariablen. Lateinische Indizes laufen dabei von 1 bis 3 , griechische über 1 und 2 . Nachfolgend nicht angegebene Formelzeichen und Operatoren werden beim erstmaligen Auftreten in der Arbeit erläutert.
Allgemein
$x^{i}$
: raumfeste kartesische Koordinaten
$\mathbf{e}_{i}$
: globale orthonormierte Basisvektoren
$\theta^{\alpha}, \theta^{3}$
: konvektive krummlinige Koordinaten
$\partial()$
: Rand eines Gebietes
()$_{L},()_{N L}$
: linear, nichtlinear
()$_{F}$
: bezogen auf den Grundzustand $F$
()$_{M}$
: bezogen auf den kritischen Zustand $M$
()$_{N}$
: bezogen auf den kritischen Zustand $N$
()$_{, \alpha}$
: partielle Ableitung
( ) $\left.\right|_{\alpha}$
: kovariante Ableitung
()
: Ableitung nach der Zeit
$\delta()$
: Variation von ( )
$d()$
: differentieller Zuwachs von ()
$\Delta()$
: Inkrement von ( )
: vorgeschriebene Größe
: dyadisches Produkt
$\delta_{j}^{i}$
: Kronecker-Delta

\section{Geometrie- und Materialkennwerte}

$\begin{array}{ll}r & : \text { Radius von Rotationsschalen (allg.) } \\ R & : \text { Radius von Kugelschalen (gemäß den Regelwerken) } \\ \phi & : \text { meridionaler Öffnungswinkel von Kugelschalen } \\ \varphi & : \text { Öffnungswinkel in Breitenkreis- bzw. Umfangsrichtung } \\ l & : \text { Länge in Axial- bzw. Meridianrichtung } \\ t & : \text { Wanddicke } \\ E & : \text { Elastizitätsmodul } \\ G & : \text { Schubmodul } \\ v & : \text { Querdehnzahl }\end{array}$




$\begin{array}{ll}\rho & : \text { Dichte } \\ \mathrm{f}_{\mathrm{y}, \mathrm{k}} & : \text { Streckgrenze } \\ \sigma_{F} & : \text { Fließspannung } \\ \kappa & : \text { Schubkorrekturfaktor } \\ r / t & : \text { Schlankheit } \\ \bar{\lambda}_{s} & : \text { bezogener Schlankheitsgrad nach DIN } 18800\end{array}$

\section{Matrizen, Vektoren}
A
: Element-, Systemmatrix
K
: Steifigkeitsmatrix
M
: Massenmatrix
$\mathbf{v}, \mathbf{z}$
: Vektor der Zustandsgrößen
p
: Vektor der Belastung

\section{Energie- und stabilitätstheoretische Größen}

$\begin{array}{ll}L & : \text { Lagrange-Funktion } \\ T & : \text { kinetische Energie } \\ \Pi & : \text { elastisches Potential, potentielle Energie } \\ \Pi_{e x t} & : \text { Potential der äußeren Kräfte } \\ \Pi_{s t r} & : \text { Formänderungsenergie } \\ \Pi_{F} & : \text { Grundenergie } \\ \Pi_{c r} & : \text { Störenergie } \\ W_{e x t} & : \text { eingeprägte Energie } \\ B^{*} & : \text { effektive Bezugsgröße } \\ \pi_{c r} & : \text { bezogene Störenergie } \\ \pi_{c r}=2,7 \% & : \text { Referenzwert der bezogenen Störenergie } \\ \eta & : \text { Stabiliätsgrad } \\ p_{c l} & : \text { ideale bzw. klassische Beullast }\end{array}$

\section{Definition}

Als Grenzlast wird allgemein die Last bezeichnet, bei deren Einwirkung ein Schalentragwerk unter bestimmten Berechnungsannahmen oder tatsächlich die Tragfähigkeit verliert. Eine Grenzlast kann somit die ideale oder reale Beullast, aber auch den mit dem Referenzwert der bezogenen Störenergie definierten Beulwiderstand repräsentieren. Letztere Bedeutung dominiert im Rahmen dieser Arbeit. 


\section{Einleitung}

Schalentragwerke werden im Maschinen- und konstruktiven Ingenieurbau eingesetzt. Ihr Tragverhalten ist von den Systemparametern abhängig. Für ausgewählte Konstellationen der Systemparameter sind die Grenzlasten von Schalentragwerken sehr empfindlich gegenüber Imperfektionen und Störungen. Sicherheitsbetrachtungen und die wirtschaftliche Bemessung erfordern eine adäquate Bewertung der Imperfektionsempfindlichkeit und der Grenzlasten von Schalentragwerken.

\subsection{Stand des Wissens}

Anfang des 20. Jahrhunderts beginnen erste Forschungsarbeiten zur Stabilität von Schalentragwerken. Die Untersuchungen zielen vorrangig auf die Ermittlung der statischen Grenzlast ab. Die sich aufzeigende Diskrepanz zwischen analytischer und experimenteller Grenzlast erfordert eine intensive Analyse der Imperfektionsempfindlichkeit und somit des Tragverhaltens von Schalentragwerken. Hierzu eröffnet die in der Mitte des letzten Jahrhunderts einsetzende Entwicklung der Methode der finiten Elemente (FEM) neue Möglichkeiten. Trotz bedeutender Fortschritte in den letzten Jahrzehnten ist die Entwicklung auf dem Gebiet der Imperfektionsempfindlichkeit sowie der statischen und kinetischen Grenzlasten von Schalentragwerken noch nicht beendet. Einige der zahlreichen Forschungsarbeiten werden nachfolgend vorgestellt.

Im Jahre 1910 unterbreitet Timoshenko [143] einen Vorschlag zur Berechnung der idealen Beullast von Kreiszylinderschalen unter Axiallast. Der Vorschlag fundiert auf der Annahme eines axialsymmetrischen Beulmusters. Flügge [50] entwickelt eine vollständige lineare Beultheorie für Kreiszylinderschalen, die eine Erfassung unsymmetrischer Beulmuster und somit eine genauere Beschreibung der idealen Beullast ermöglicht. Eine Alternative hierzu stellt Donnell [35] vor. Diese ist u. a. dadurch gekennzeichnet, dass die Verschiebung in Radialrichtung als klein gegenüber deren Ableitung nach den Koordinaten angesehen wird, was aber bei kleinen Umfangswellenzahlen zur Überschätzung der idealen Beullast führen kann [116]. Eine Bewertung des Verzweigungs- und Anfangs-Nachbeul-Verhaltens von Schalentragwerken wird mit der wegweisenden Arbeit von Koiter [88] möglich. Ein Modell zur Approximation des Nachbeulminimums perfekter, theoretisch unendlich langer Kreiszylinder- 
schalen präsentieren v. Kármán und Tsien [146]. Eßlinger und Geier [45] untersuchen den Einfluss realer Randbedingungen auf das Nachbeulminimum. Eßlinger [44] stellt die signifikante Bedeutung der Membrankräfte für die Imperfektionsempfindlichkeit heraus. Im Einklang mit dieser Erkenntnis entwickeln Fritz und Wittek [51] das Konzept der quasi-verzerrungsfreien Verbiegungen, mit dem eine sehr konservative Abschätzung des Nachbeulminimums gelingt. Als Weiterentwicklungen dieses Konzeptes sind die Lower-Bound-Theory von Croll und Batista [26] sowie das Konzept der richtungsorientiert reduzierten Membranbeulung von Wittek und Grote [161] zu interpretieren. Die drei Konzepte basieren auf der Vernachlässigung bestimmter Membrananteile im elastischen Potential und sind Grundlage umfangreicher Untersuchungen. Kröplin et al. [96] entwickeln das Störenergie-Konzept. Wagenhuber [151], Tranel [144], Schäfer [124] und Spohr [139] analysieren mit dem Störenergie-Konzept die Grenzlasten verschiedenartig belasteter Kreiszylinderschalen. Herber [72], Steinhardt und Schulz [140] sowie Krysik [97] geben Beullasten von Kreiszylinderschalen unter Axiallast und Radialinnendruck an. Düsing [37] erörtert das Stabilitätsverhalten längsversteifter stählerner Kreiszylinderschalen unter Axiallast. Guggenberger [62] bewertet die Beullast diskret gelagerter Kreiszylinderschalen unter Axiallast. Geier et al. [57] und Hühne et al. [80] untersuchen die Grenztragfähigkeit axialgedrückter Kreiszylinderschalen aus Faserverbundwerkstoff. Für den gleichen Beulfall diskutieren Obrecht et al. [113] den Einfluss auxetischen Materialverhaltens auf die Beullast.

Im Jahre 1915 ermittelt Zoelly [169] die ideale Beullast von Kugelschalen unter Radialdruck. Eine nichtlineare Schalentheorie zur Analyse der Stabilität dieses Beulfalles verwenden v. Kármán und Tsien [145]. Die Untersuchungen basieren auf der Annahme, dass symmetrisches Durchschlagen den Beulvorgang charakterisiert und die Beullast eines Kugelschalensegmentes der einer Vollkugel entspricht. Experimente von Klöppel und Jungbluth [85] zeigen, dass das Beulen von Kugelschalen unter Radialdruck ausgehend von einer lokalen Imperfektion unsymmetrisch erfolgen kann. Für den Fall unsymmetrischen Beulens ermittelt Huang [76] die Beullast eingespannter Kugelschalen. Weinitschke [155] untersucht ferner das Stabilitätsverhalten unverschieblich gelenkig gelagerter Kugelschalen. Aus beiden Arbeiten folgt, dass rotationssymmetrisches Beulen von Kugelschalen unter Radialdruck nur für ausgewählte Werte des in Gl. (6.2) definierten Parameters $\lambda$ zu erwarten ist. Fitch und Budiansky [48] analysieren Kugelschalen unter rotationssymmetrischer Teilflächenbelastung. Den Einfluss von Imperfektionen auf die Durchschlaglast eingespannter Kugelschalen bewertet Wessels [158]. Kollár [90] gibt einen Überblick über Experimente und Vorschläge zur Abschätzung des Nachbeulminimums. Funktionen zur Beschreibung experimentell ermittelter Beullasten werden von Samuelson und Eggwertz [122] definiert. Wunderlich et al. [162] analysieren den Einfluss des meridionalen Öffnungswinkels und verschiedener Randbedingungen auf die Beullast von Kugelschalen. 
Blachut und Galletly [14] erörtern die Bemessung von Kugelschalen mit kleinen Abflachungen und veränderlicher Wanddicke. Der Einfluss von Öffnungen im Scheitelbereich auf die Beullast von Kugelschalen wird von Kubor [98] untersucht.

Im Jahre 1937 veröffentlicht Pflüger [115] eine Arbeit, in welcher in Anlehnung an die Beultheorie nach Flügge [50] die Stabilität von Kegelstumpfschalen, kurz Kegelschalen, unter Axiallast beschrieben wird. Aufgrund der Komplexität des zugrunde liegenden Differentialgleichungssystems gelingt dessen Lösung nur für den Fall einer linear veränderlichen Wanddicke. Dahingegen ermittelt Seide [132] in Anlehnung an die Beultheorie nach Donnell [35] und unter Annahme symmetrischen Beulens eine Näherung für die ideale Beullast axialgedrückter Kegelschalen mit konstanter Wanddicke. Für den Fall unsymmetrischen Beulens kann die ideale Beullast anhand der Arbeiten von Schnell [129] sowie Mushtari und Sachenkov [108] abgeschätzt werden. Schiffner [125] analysiert auf Grundlage einer nichtlinearen Schalentheorie den Einfluss ausgewählter Randbedingungen und des meridionalen Öffnungswinkels auf die Stabilität von Kegelschalen unter Axiallast. Ender [43] zeigt die Ähnlichkeit des Nachbeulverhaltens axialgedrückter Kegel- und Kreiszylinderschalen auf. Mit dem Störenergie-Konzept bewerten Wagenhuber [151] und Knoke [87] die Imperfektionsempfindlichkeit und die Grenzlasten von Kegelschalen unter Axiallast. Zahlreiche Experimente zur Beullast axialgedrückter Kegelschalen gehen, wie in [97] detailliert dokumentiert, auf Seide, Weingarten und Morgan zurück. Krysik [97] führt experimentelle und numerische Untersuchungen zur Stabilität von Kegelschalen unter Axiallast und Innendruck durch. Die Stabilität flüssigkeitsgefüllter Kegelschalen ist Gegenstand der Experimente von Vandepitte et al. [148]. Goldfeld et al. [61] widmen sich der Stabilität von Kegelschalen aus Faserverbundwerkstoff.

Im Jahre 1968 präsentiert Giesen [59] Beiträge verschiedener Autoren zum Tragverhalten von Naturzug-Kühltürmen. Zerna et al. [164] bewerten das Beulverhalten hyperbolischer Rotationsschalen unter Axiallast und Manteldruck und verdeutlichen den Einfluss von Meridianform, Randbedingungen und Wanddicke. Ähnliche Untersuchungen führt Weise [156] für Rotationsschalen unter tangentialer Randlast durch. Eckstein et al. [39] geben für schwach gekrümmte Rotationsschalen unter Axiallast und Manteldruck wirklichkeitsnahe Beullasten an. Zerna und Mungan [165] untersuchen das Tragverhalten von Schalentragwerken mit negativer Gauß'scher Krümmung.

Im Jahre 1959 zeigt Galletly [53] auf, dass Instabilität bei torisphärischen Schalen nicht nur unter Außendruck, sondern auch bei Innendruck eintreten kann. Für diese Beulfälle werden numerische Untersuchungen zur Stabilität u.a. von Thurston und Holston [142] sowie von Rensch [119] durchgeführt. Die Stabilität torisphärischer und torikonischer Schalen unter Außen- bzw. Innendruck wird von Wunderlich et al. [163] und Albertin [2] analysiert. Für torisphärische Schalen unter Außendruck ge- 
ben Galletly et al. [54] experimentell ermittelte Beullasten an. Beullasten zusammengesetzter Kreiszylinder-Kegelschalen unter Axiallast und Außendruck werden von Swadlo [141] sowohl experimentell als auch numerisch ermittelt. Knoke [87] untersucht die Imperfektionsempfindlichkeit und die Grenzlasten axialgedrückter zusammengesetzter Kreiszylinder-Kegelschalen mit Hilfe des Störenergie-Konzeptes.

Im Jahre 1954 diskutieren Hoff und Bruce [74] anhand der potentiellen Energie die Stabilität flacher Bögen unter kinetischer Belastung. Budiansky und Roth [24] ermitteln die kinetische Beullast für den Fall rotationssymmetrischen Beulens flacher Kugelschalen. Dahingegen analysieren Fulton und Barton [52] den Fall unsymmetrischen Stabilitätsversagens. Beide Arbeiten basieren auf der Bewertung der zeitlichen Entwicklung charakteristischer Verformungen und bilden die Grundlage zahlreicher Untersuchungen zur Stabilität von Kugelschalen unter zeitveränderlicher Belastung. Akkas [1] veröffentlicht Ergebnisse zum unsymmetrischen Beulen eingespannter flacher Kugelschalen. Kao und Perrone [83] widmen sich in ihrer Arbeit dem rotationssymmetrischen Beulen unterschiedlich belasteter imperfekter Kugelschalen. Der Einfluss der Belastungsgeschwindigkeit auf das Durchschlagverhalten von Kugelschalen unter Radialdruck wird von Wessels [158] aufgezeigt. Burmeister [25] untersucht für verschiedene Belastungen den Einfluss des Materialverhaltens und der Aussteifung von Öffnungen im Scheitelbereich auf die kinetische Stabilität von Kugelschalen. Dinkler und Kröplin [33] bewerten die Stabilität von Grundzuständen gegenüber kinetischen Störungen mittels des Stabilitätsgrades. Eine Bewertung der kinetischen Stabilität von Systemen, für die kein Potential existiert, ist mit Hilfe von Ljapunow-Exponenten möglich, vgl. Malkin [104], Bolotin [17], Leipholz [102] und Kreuzer [94]. Ljapunow-Exponenten werden u. a. in Arbeiten des Sonderforschungsbereichs 151 [133] verwendet, siehe auch Krätzig et al. [92]. Ewert et al. [47] analysieren mittels Ljapunow-Exponenten und der Sensitivität [149] die Stabilität von Grundzuständen gegenüber kinetischen Störungen. Dahingegen erörtern Heinen et al. [69] die kinetische Stabilität von Kreiszylinderschalen unter stoßartiger Axiallast anhand von Stabilitätsgleichungen, die mit Hilfe von Reihenansätzen für die Störverschiebungen in gewöhnliche Differentialgleichungen überführt werden und eine Abschätzung der kinetischen Grenzlast ermöglichen.

Die Analyse der Imperfektionsempfindlichkeit umfasst die Identifikation ihrer Ursache sowie Methoden zu ihrer Bewertung und Verringerung. Zur Bewertung der Imperfektionsempfindlichkeit sind verschiedene Methoden geeignet. Bei Interpretation von Imperfektionsempfindlichkeit als grundzustandspezifische Systemeigenschaft ist das Störenergie-Konzept anwendbar. Andernfalls sind anfängliche Imperfektionen bei der Analyse des Stabilitätsverhaltens zu berücksichtigen. Dieses Vorgehen ist zwar unabhängig von der Imperfektionsempfindlichkeit eines Schalentragwerkes möglich, erlaubt aber keinen direkten quantitativen Vergleich der Imperfekti- 
onsempfindlichkeit verschiedener Beulfälle. Schwierig ist die Wahl der anzusetzenden Imperfektionen, da eine Imperfektion und die zugehörige Grenzlast unmittelbar miteinander verknüpft sind. Zum Einfluss einzelner Imperfektionen auf die statische Grenzlast und zur Wahl ungünstiger Imperfektionen gibt es zahlreiche Untersuchungen. Der häufig verwendete Ansatz der skalierten ersten Beuleigenform des perfekten Systems als ungünstige Imperfektion wird u. a. von Wagenhuber [151] analysiert. Albertin [2] bewertet den Einfluss lokaler Einzelbeulen. Jürke et al. [82] untersuchen den Einfluss von axialsymmetrischen wulstartigen Imperfektionen. Die Stabilität einer axialgedrückten Kreiszylinderschale, die eine Rundschweißnaht aufweist, wird von Bornscheuer et al. [18] bewertet. Ein Konzept zur Ermittlung einer ungünstigen Imperfektion und der zugehörigen Grenzlast wird von Deml [30] vorgestellt. Marcinowski [106] leitet aus dem Verzweigungsverhalten eingespannter Kugelschalen unter Radialdruck ungünstige Imperfektionen her. Reale Imperfektionen setzen sich aus einzelnen Imperfektionen zusammen. Bolotin erörtert die Anwendung probabilistischer Methoden zur Beschreibung des Zusammenhanges zwischen Imperfektionen und Grenzlast, vgl. [41]. Dieses Vorgehen erfordert genügend Informationen hinsichtlich realistischer Imperfektionsspektren. Die Informationen können mit der von Arbocz und Abramovich [7] vorgestellten Imperfektionsdatenbank bereitgestellt werden. Elishakoff [41] diskutiert die Einbeziehung des ImperfektionssensitivitätsKonzeptes in die Bemessung von Tragwerken. Eine weitere Möglichkeit zur Erfassung des Einflusses verschiedener Imperfektionen auf die Grenzlasten stellen Ersatzimperfektionen dar, vgl. Winterstetter und Schmidt [160]. Für Kreiszylinderschalen unter Axiallast und Radialdruck werden von Schneider und Gettel [127, 128] Empfehlungen zur Wahl geometrischer Ersatzimperfektionen gegeben.

Von praktischer Relevanz ist neben der Bewertung auch die Verringerung der Imperfektionsempfindlichkeit von Schalentragwerken. Diese bedingt eine Modifikation von Systemparametern und somit u.U. eine signifikante Änderung des Systemverhaltens. Reitinger [118] stellt eine Optimierungsmethode vor, die auf der direkten Berechnung singulärer Punkte mit erweiterten Systemen und einer effizienten Sensitivitätsanalyse basiert. Die Optimierung von Tragwerken hinsichtlich der Imperfektionsempfindlichkeit bzw. statischen Grenzlast ist Gegenstand der Arbeiten von Gerdes [58] und Goldfeld et al. [61]. Mang et al. [105] erörtern die Verringerung der Imperfektionsempfindlichkeit von Systemen mit symmetrischem Verzweigungsverhalten.

Die Entwicklung von Regelwerken zum Nachweis der Stabilität von Schalentragwerken folgt dem Stand des Wissens in der Regel etwas verzögert. So sind erst im Jahre 1968 mit der NASA SP-8007 [109] und der NASA SP-8019 [110] entsprechende Regelwerke für die Luft- und Raumfahrttechnik verfügbar. Für den konstruktiven Ingenieurbau wurden neben speziellen Fachnormen die DASt-Richtlinie 013 [28], die ECCS-Recommendations [38], die DASt-Richtlinie 017 [29], die DIN 18800 [31] so- 
wie der Eurocode 3 [46] erarbeitet. Diese Regelwerke beziehen sich primär auf stählerne Schalentragwerke unter statischen Belastungen. Für Schalentragwerke aus modernen Faserverbundwerkstoffen und für Schalentragwerke unter kinetischen Belastungen sind dem Autor keine Regelwerke zum Nachweis der Stabilität bekannt.

\subsection{Ziel der Arbeit}

Ziel der Arbeit ist die Analyse der Imperfektionsempfindlichkeit und der Grenzlasten von Schalentragwerken mit Hilfe des Störenergie-Konzeptes. Dabei sind insbesondere Schalentragwerke aus Stahl und mit unterschiedlichen Gauß'schen Krümmungen, verschiedenen Randbedingungen und Belastungen zu untersuchen. Mit den Ergebnissen werden die Grenzlasten verschiedener Regelwerke bewertet und Anregungen für deren zukünftige Entwicklung erarbeitet. Ferner sind die Imperfektionsempfindlichkeit von Schalentragwerken aus Faserverbundwerkstoff sowie die Verallgemeinerung des Störenergie-Konzeptes zu erörtern. Des Weiteren wird eine Möglichkeit zur Modifikation der Imperfektionsempfindlichkeit von Schalentragwerken vorgestellt. 


\section{Berechnungsmodell}

Die Bewertung des Tragverhaltens von Schalentragwerken erfordert die Beschreibung des Spannungs- und Verformungszustandes des Schalenkontinuums. Hierzu sind verschiedene Variationsprinzipien und Schalentheorien geeignet, wobei deren Wahl die Effizienz von Verfahren zur Lösung der Anfangs-Randwertaufgabe beeinflussen kann.

\subsection{Kontinuumsmechanische Grundlagen}

Bei Interpretation des Schalenkontinuums als nicht polares Kontinuum besitzt jeder materielle Punkt nur Verschiebungsfreiheitsgrade. Ferner treten keine flächen- und volumenbezogenen Momentenbelastungen auf. Der Spannungs- und der Verformungszustand sind mittels Kinematik, Bilanz- und konstitutiver Gleichungen beschreibbar.

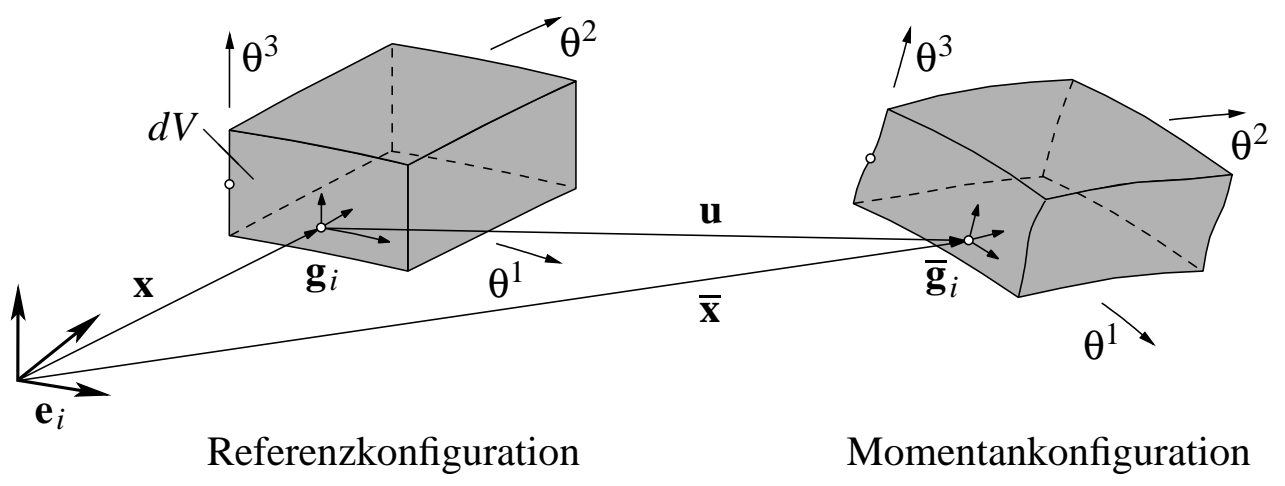

Bild 2.1: Geometrie und Kinematik des Schalenkontinuums

Das undeformierte spannungsfreie Schalenkontinuum repräsentiert die Referenzkonfiguration. In dieser Konfiguration ist ein materieller Punkt mit dem Ortsvektor $\mathbf{x}\left(\theta^{i}\right)$ zu spezifizieren. In der Momentankonfiguration charakterisiert der Ortsvektor $\overline{\mathbf{x}}\left(\theta^{i}\right)$ die Position des Punktes. Die kovarianten Basisvektoren des konvektiven Koordinatensystems $\theta^{i}$ sind in der Referenz- bzw. Momentankonfiguration definiert zu

$$
\mathbf{g}_{i}=\frac{\partial \mathbf{x}}{\partial \theta^{i}} \quad \text { bzw. } \quad \overline{\mathbf{g}}_{i}=\frac{\partial \overline{\mathbf{x}}}{\partial \theta^{i}} .
$$

Mit den kovarianten Basisvektoren und den Dualitäten

$$
\mathbf{g}^{i} \cdot \mathbf{g}_{j}=\delta_{j}^{i} \quad \text { und } \quad \overline{\mathbf{g}}^{i} \cdot \overline{\mathbf{g}}_{j}=\delta_{j}^{i}
$$


sind in beiden Konfigurationen die kontravarianten Basisvektoren und der Metriktensor

$$
\mathbf{g}=g_{i j} \mathbf{g}^{i} \otimes \mathbf{g}^{j}=g^{i j} \mathbf{g}_{i} \otimes \mathbf{g}_{j} \quad \text { bzw. } \quad \quad \overline{\mathbf{g}}=\bar{g}_{i j} \overline{\mathbf{g}}^{i} \otimes \overline{\mathbf{g}}^{j}
$$

zu ermitteln. Der Übergang von der Referenzkonfiguration in die Momentankonfiguration ist mit dem Verschiebungsvektor bzw. dem Deformationsgradienten,

$$
\mathbf{u}=\overline{\mathbf{x}}-\mathbf{x}=u_{i} \mathbf{g}^{i} \quad \text { bzw. } \quad \mathbf{F}=\overline{\mathbf{g}}_{i} \otimes \mathbf{g}^{i},
$$

beschreibbar. Mit Hilfe des Deformationsgradienten ist als objektives Verzerrungsmaß der auf die Referenzkonfiguration bezogene Green-Lagrange-Verzerrungstensor

$$
\mathbf{E}=E_{i j} \mathbf{g}^{i} \otimes \mathbf{g}^{j}=\frac{1}{2}\left(\mathbf{F}^{T} \cdot \mathbf{F}-\mathbf{g}\right)=\frac{1}{2}\left(\bar{g}_{i j}-g_{i j}\right) \mathbf{g}^{i} \otimes \mathbf{g}^{j}
$$

definiert. Dem Verzerrungstensor ist der zweite Piola-Kirchhoff-Spannungstensor

$$
\mathbf{S}=S^{i j} \mathbf{g}_{i} \otimes \mathbf{g}_{j}=\operatorname{det} \mathbf{F} \sigma^{i j} \mathbf{g}_{i} \otimes \mathbf{g}_{j}
$$

energetisch konjugiert. Die Komponenten des Piola-Kirchhoff-Spannungstensors sind mit der Determinante des Deformationsgradienten in die Komponenten des in der Momentankonfiguration definierten Cauchy-Spannungstensors $\boldsymbol{\sigma}$ transformierbar.

Bilanzgleichungen besitzen axiomatischen Charakter, fordern für jede Konfiguration die Erhaltung der Masse, des Impulses und Drehimpulses und ermöglichen Aussagen zur Energie eines Systems [4]. Die Massebilanz ist bei Schalentragwerken a priori erfüllt. Impuls- und Drehimpulsbilanz beschreiben das Gleichgewicht des Schalenkontinuums. Aus der Drehimpulsbilanz resultiert die Symmetrie des Cauchy-Spannungstensors. Die Energiebilanz folgt aus dem ersten Hauptsatz der Thermodynamik und ist in der Referenzkonfiguration für isotherme reversible Prozesse mit

$$
\frac{d}{d t} \frac{1}{2} \int_{V} \rho \dot{u}_{i} \dot{u}_{j} g^{i j} d V+\int_{V} S^{i j} \dot{E}_{i j} d V=\int_{V} \dot{u}_{i} b^{i} d V+\int_{\partial V_{S}} \dot{u}_{i} \widehat{t}^{i} d(\partial V)
$$

gegeben. Die Energiebilanz fordert Identität zwischen der zeitlichen Änderung der Gesamtenergie eines Systems und der Leistung der äußeren Kräfte. Die Gesamtenergie besteht aus kinetischer Energie und Formänderungsenergie. Die äußeren Kräfte umfassen volumenbezogene Kräfte $b^{i}$ und Oberflächenspannungen $\widehat{t}^{i}$.

Die konstitutiven Gleichungen formulieren den Zusammenhang zwischen Spannungen und Verzerrungen und sind für linear elastisches, isotropes Materialverhalten mit

$$
S^{i j}=C^{i j k l} E_{k l}, \text { mit } C^{i j k l}=\lambda g^{i j} g^{k l}+\mu\left(g^{i k} g^{j l}+g^{i l} g^{j k}\right),
$$

definiert. Die den symmetrischen Elastizitätstensor $\mathbf{C}$ charakterisierenden Lamé-Konstanten $\lambda$ und $\mu$ sind mit den Materialparametern $E$ und $v$ festgelegt [9]. Kinematik, Bilanz- und konstitutive Gleichungen zählen zu den Modellgleichungen. 
Die Modellgleichungen bilden mit den Anfangs- und Randbedingungen der Bewegung des Schalenkontinuums eine Anfangs-Randwertaufgabe, die zur Lösung mit der FEM als Variationsaufgabe formulierbar ist. Für dynamische Systeme ist die Variationsaufgabe mit dem Hamilton-Prinzip beschrieben,

$$
\delta \int_{t_{a}}^{t_{b}} L d t=0 \text {, mit } L=T-\left(\Pi_{s t r}+\Pi_{e x t}\right) .
$$

Das Hamilton-Prinzip fordert die Stationarität des Integrals der Lagrange-Funktion $L$ über ein beliebiges Zeitintervall und gilt für verschiedene Formulierungen der potentiellen Energie sowie Systeme mit monogenetischen Kräften und holonomen Randund Nebenbedingungen [100, 159]. Für Systeme mit polygenetischen Kräften, z.B. Dämpfungskräften, ist die Variation der Lagrange-Funktion um die entsprechenden Arbeiten zu ergänzen [111]. Bei konservativen statischen Systemen ist die gegebene Randwertaufgabe mit dem Prinzip vom Stationärwert des Gesamtpotentials,

$$
\Pi\left(u_{i}\right)=\frac{1}{2} \int_{V} E_{i j} C^{i j k l} E_{k l} d V-\int_{V} u_{i} b^{i} d V-\int_{\partial V_{S}} u_{i} \widehat{t}^{i} d(\partial V) \rightarrow \text { stat. , }
$$

beschreibbar. Für dieses Prinzip sind die Euler-Gleichungen mit den Gleichgewichtsbedingungen und die natürlichen Randbedingungen mit den statischen Randbedingungen gegeben. Das Prinzip setzt als Nebenbedingungen die Erfüllung der konstitutiven Gleichungen und die Erfüllung der Kinematik im Schalenkontinuum und auf dessen Rand voraus. Das Prinzip ist Ausgangspunkt zur Herleitung finiter Elemente in Weggrößenformulierung. Die Nebenbedingungen limitieren aber den Raum zulässiger Ansatzfunktionen. Daher ist die Definition erweiterter Prinzipien sinnvoll. So folgt aus dem Prinzip (2.10) mit einer Legendre-Transformation bezüglich der Formänderungsenergiedichte und unter Berücksichtigung der kinematischen Randbedingungen im Funktional $\Pi$ das Prinzip von Hellinger/Reissner [70, 117],

$$
\begin{aligned}
\Pi\left(S^{i j}, u_{i}\right) & =\int_{V} S^{i j} E_{i j} d V-\frac{1}{2} \int_{V} S^{i j}\left(C^{i j k l}\right)^{-1} S^{k l} d V \\
& -\int_{V} u_{i} b^{i} d V-\int_{\partial V_{S}} u_{i} \widehat{t}^{i} d(\partial V)-\int_{\partial V_{u}}\left(u_{i}-\widehat{u}_{i}\right) t^{i} d(\partial V) \rightarrow \text { stat. }
\end{aligned}
$$

Primäre Variablen dieses erweiterten Prinzips sind die Spannungen und die Verschiebungen. Die Euler-Gleichungen des Prinzips umfassen die Gleichgewichtsbedingungen und die Kinematik. Natürliche Randbedingungen sind die statischen und kinematischen Randbedingungen. Zweifeldprinzip (2.11) ist Ausgangspunkt zur Herleitung finiter Elemente in gemischter Formulierung und kann auch aus dem Prinzip vom Stationärwert des komplementären Gesamtpotentials und dem Prinzip von $\mathrm{Hu}$ Washizu [75, 154] hergeleitet werden. Das Prinzip von Hu-Washizu ist ein sehr allge- 
meingültiges Variationsprinzip, da dessen Euler-Gleichungen sämtliche Modellgleichungen beinhalten und dessen natürliche Randbedingungen die statischen und die kinematischen Randbedingungen umfassen. Die unabhängigen Variablen sind dabei die Verschiebungen, Verzerrungen, Spannungen und Oberflächenspannungen. Das Prinzip von Hu-Washizu ist aufgrund der oft schwierigen Wahl geeigneter Ansatzfunktionen nur bedingt für eine numerische Umsetzung geeignet und dient häufig der variationellen Begründung von Methoden zur Verringerung von Versteifungseffekten.

\subsection{Schalentheorien}

Zur Berechnung von Schalentragwerken mit klassischen Schalenelementen ist die Lagrange-Funktion anhand von Zustandsgrößen, die bezüglich einer Referenzfläche definiert sind, zu beschreiben. Hierzu sind verschiedene Schalentheorien geeignet [22]. Im Rahmen dieser Arbeit werden eine 5- und eine 3-Parameter-Schalentheorie verwendet. Die Gültigkeit der Dünne-Hypothese [8] wird vorausgesetzt; Referenzfläche ist die Schalenmittelfläche. In der Referenzkonfiguration ist auf der Schalenmittelfläche, und wie in Bild 2.2 aufgezeigt, die kovariante Basis mit

$$
\mathbf{a}_{\alpha}=\mathbf{r}_{, \alpha} \quad \text { und } \quad \mathbf{a}_{3}=\frac{\mathbf{a}_{1} \times \mathbf{a}_{2}}{\left|\mathbf{a}_{1} \times \mathbf{a}_{2}\right|}
$$

definiert. Kontravariante Basisvektoren $\mathbf{a}^{i}$ sind entsprechend Dualität (2.2) zu ermitteln. Mit den Basisvektoren und deren Ableitungen sind die Komponenten des Metrik- und Krümmungstensors sowie die Christoffelsymbole zweiter Art festgelegt,

$$
\begin{aligned}
& a_{\alpha \beta}=\mathbf{a}_{\alpha} \cdot \mathbf{a}_{\beta}, a_{\alpha \rho} a^{\rho \beta}=\delta_{\alpha}^{\beta}, \\
& b_{\alpha \beta}=\mathbf{a}_{\alpha, \beta} \cdot \mathbf{a}_{3}, b_{\beta}^{\alpha}=b_{\beta \rho} a^{\rho \alpha}, \Gamma_{\alpha \beta}^{\lambda}=\mathbf{a}^{\lambda} \cdot \mathbf{a}_{\alpha, \beta} .
\end{aligned}
$$

Entsprechende differentialgeometrische Größen sind für die Momentankonfiguration definiert. Der Verschiebungsvektor $\mathbf{u}=u_{i} \mathbf{a}^{i}$ beschreibt die Änderung der Position eines Punktes der Schalenmittelfläche beim Übergang von der Referenzkonfiguration in die Momentankonfiguration. Dabei auftretende Rotationen des inextensiblen Direktors $\mathbf{d}$ werden mit dem Differenzvektor $\mathbf{w}=\overline{\mathbf{d}}-\mathbf{a}_{3}=w_{\alpha} \mathbf{a}^{\alpha}$ erfasst.

Mit den differentialgeometrischen Größen (2.13) folgen bei Vernachlässigung der von $\left(\theta^{3}\right)^{2}$ abhängigen Verzerrungsanteile sowie der Normalverzerrungen in Richtung der Schalennormalen $\mathbf{a}_{3}$ die Komponenten des Green-Lagrange-Verzerrungstensors zu

$$
E_{i j}=\left[\begin{array}{ll}
E_{\alpha \beta} & E_{\alpha 3} \\
E_{3 \alpha} & E_{33}
\end{array}\right]=\left[\begin{array}{cc}
\alpha_{\alpha \beta}+\theta^{3} \beta_{\alpha \beta} & 0,5 \gamma_{\alpha} \\
0,5 \gamma_{\alpha} & 0
\end{array}\right],
$$

mit den Membranverzerrungen

$$
\alpha_{\alpha \beta}=\frac{1}{2}\left(\left.u_{\alpha}\right|_{\beta}+\left.u_{\beta}\right|_{\alpha}-2 b_{\alpha \beta} u_{3}\right)+\frac{1}{2}\left(u_{3, \alpha}+b_{\alpha}^{\lambda} u_{\lambda}\right)\left(u_{3, \beta}+b_{\beta}^{\rho} u_{\rho}\right),
$$




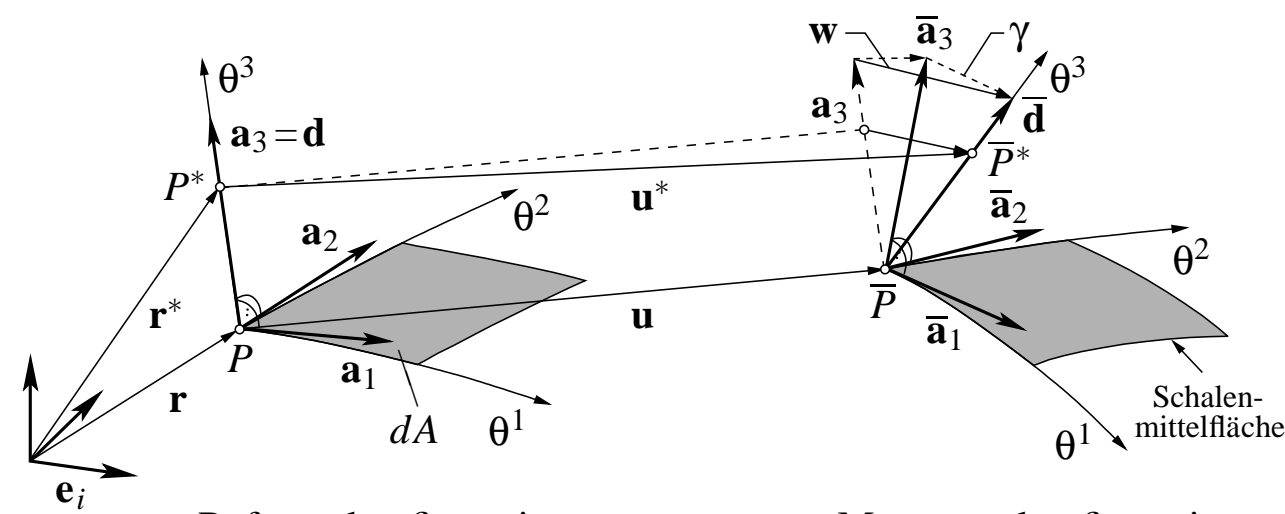

Referenzkonfiguration

Momentankonfiguration

Bild 2.2: Kinematik des Schalenkontinuums [8]

den Verkrümmungen

$$
\beta_{\alpha \beta}=\frac{1}{2}\left(\left.w_{\alpha}\right|_{\beta}+\left.w_{\beta}\right|_{\alpha}-\left.b_{\alpha}^{\lambda} u_{\lambda}\right|_{\beta}-\left.b_{\beta}^{\lambda} u_{\lambda}\right|_{\alpha}+2 b_{\alpha}^{\lambda} b_{\lambda \beta} u_{3}\right)
$$

und den Querschubverzerrungen

$$
\gamma_{\alpha}=w_{\alpha}+u_{3, \alpha}+b_{\alpha}^{\lambda} u_{\lambda} .
$$

Die Kinematik des Schalenkontinuums wird somit im Sinne einer Theorie mäßiger Rotationen beschrieben. Diese Approximationsstufe ist für baupraktisch relevante Aufgabenstellungen als ausreichend anzusehen. Ferner sind sowohl die Formänderungsenergiedichte als auch die Spannungen und deren statisches Moment bezüglich der Schalenmittelfläche über die Wanddicke zu integrieren. Hierbei wird die Normalspannung senkrecht zur Schalenmittelfläche identisch null gesetzt. Des Weiteren werden entsprechend der ersten Approximation der Schalentheorie die Determinante des Schalenshifters zu eins und die Komponenten des Metriktensors, die einem Punkt $P^{*}$ des Schalenkontinuums zugehörig sind, mit $a_{\alpha \beta}^{*}=a_{\alpha \beta}$ angesetzt [8]. Aus der Integration resultieren die Komponenten des Elastizitätstensors,

$$
H^{\alpha \beta \rho \lambda}=\frac{1-v}{2}\left(a^{\alpha \rho} a^{\beta \lambda}+a^{\alpha \lambda} a^{\beta \rho}+\frac{2 v}{1-v} a^{\alpha \beta} a^{\rho \lambda}\right),
$$

die Dehn-, Biege- und Schubsteifigkeit,

$$
D=\frac{E t}{1-v^{2}}, B=\frac{E t^{3}}{12\left(1-v^{2}\right)}, S=\kappa \frac{E t}{2(1+v)}, \text { mit } \kappa=5 / 6,
$$

sowie die auf die kovariante Basis bezogenen Querkräfte $q^{\alpha}$, Momente $m^{\alpha \beta}$ und unsymmetrischen Membrankräfte $n_{u}^{\alpha \beta}$. Die Membrankräfte sind aufgrund des geforderten Momentengleichgewichtes um die Schalennormale $\mathbf{a}_{3}$ gemäß

$$
n^{\alpha \beta}=n_{u}^{\alpha \beta}+m^{\alpha \rho} b_{\rho}^{\beta}
$$


symmetrisierbar. Die symmetrischen Membrankräfte sind den Membranverzerrungen (2.15) energetisch konjugiert. Entsprechendes gilt für die Momente und die Verkrümmungen sowie für die Querkräfte und die Querschubverzerrungen.

Die 5-Parameter-Schalentheorie, bei der die Kinematik des Schalenkontinuums mit fünf geometrischen Parameter beschrieben wird, basiert auf der Reissner-MindlinTheorie. Hier folgt das Prinzip vom Stationärwert des Gesamtpotentials zu

$$
\begin{aligned}
\Pi\left(u_{i}, w_{\beta}\right) & =\frac{1}{2} \int_{A}\left(\alpha_{\alpha \beta} D H^{\alpha \beta \rho \lambda} \alpha_{\rho \lambda}+\beta_{\alpha \beta} B H^{\alpha \beta \rho \lambda} \beta_{\rho \lambda}+\gamma_{\alpha} S a^{\alpha \beta} \gamma_{\beta}\right) d A \\
& -\int_{A} u_{i} \widehat{p}^{i} d A-\int_{\partial A_{S}}\left(u_{i} \widehat{n}^{i}+w_{\beta} \widehat{m}^{\beta}\right) d(\partial A) \rightarrow \text { stat. }
\end{aligned}
$$

Die wesentlichen Randbedingungen sind

$$
u_{i}-\widehat{u}_{i}=0 \text { und } w_{\beta}-\widehat{w}_{\beta}=0 \text { auf dem Rand } \partial A_{u} .
$$

Die eingeprägten Kraftgrößen $\widehat{n}^{i}$ und $\widehat{m}^{\beta}$ wirken auf dem Rand $\partial A_{S}$ und sind für den Fall, dass die Schalenränder mit den Hauptkrümmungslinien und den Koordinatenlinien $\theta^{\alpha}$ übereinstimmen, folgenden inneren Kraftgrößen zugeordnet,

$$
n^{\beta}=v_{\alpha} n_{u}^{\alpha \beta}, n^{3}=v_{\alpha} q^{\alpha}, m^{\beta}=v_{\alpha} m^{\alpha \beta} .
$$

Dabei repräsentiert $v_{\alpha}$ die Komponenten des Randnormalenvektors. Eine allgemeinere Darstellung des Potentials der eingeprägten Randkraftgrößen ist in [8] aufgezeigt. Das Prinzip von Hellinger/Reissner [66] folgt für die 5-Parameter-Schalentheorie unter Berücksichtigung der kinematischen Beziehung (2.17) und der Bedingungsgleichungen für das Momentengleichgewicht um die kontravarianten Basisvektoren $\mathbf{a}^{\beta}$ sowie mit den eingeprägten Membranverzerrungen $\widehat{\alpha}_{\alpha \beta}$ und Verkrümmungen $\widehat{\beta}_{\alpha \beta} \mathrm{zu}$

$$
\begin{aligned}
\Pi & =\Pi\left(n^{\alpha \beta}, m^{\alpha \beta}, u_{i}\right)=\int_{A}\left\{\left(n^{\alpha \beta}-m^{\rho \beta} b_{\rho}^{\alpha}\right) \alpha_{\alpha \beta}+\left.m^{\alpha \beta}\right|_{\alpha}\left(b_{\beta}^{\lambda} u_{\lambda}+\left.u_{3}\right|_{\beta}\right)\right\} d A \\
& -\frac{1}{2} \int_{A}\left(\frac{1}{D} n^{\alpha \beta} F_{\alpha \beta \rho \lambda} n^{\rho \lambda}+\frac{1}{B} m^{\alpha \beta} F_{\alpha \beta \rho \lambda} m^{\rho \lambda}+\left.\left.\frac{1}{S} m^{\rho \alpha}\right|_{\rho} a_{\alpha \beta} m^{\lambda \beta}\right|_{\lambda}\right) d A \\
& -\int_{A}\left(u_{i} \widehat{p}^{i}+n^{\alpha \beta} \widehat{\alpha}_{\alpha \beta}+m^{\alpha \beta} \widehat{\beta}_{\alpha \beta}\right) d A+\int_{\partial A}\left(\widehat{w}_{\beta} m^{\beta}-u_{i} \widehat{n}^{i}\right) d(\partial A) \\
& +\int_{\partial A_{S}} w_{\beta}\left(m^{\beta}-\widehat{m}^{\beta}\right) d(\partial A) \rightarrow \text { stat. },
\end{aligned}
$$

wobei $F_{\alpha \beta \rho \lambda}$ die Komponenten des inversen Elastizitätstensors darstellt. Die wesentlichen Randbedingungen sind gegeben mit

$$
u_{i}-\widehat{u}_{i}=0 \text { auf } \partial A_{u} \text { und } m^{\beta}-\widehat{m}^{\beta}=0 \text { auf } \partial A_{S} .
$$


Somit entfällt bei schubsteifen Schalentragwerken im Potential (2.24) das Integral über den Rand $\partial A_{S}$. Wird dagegen die Gültigkeit der Kirchhoff-Love-Hypothese vorausgesetzt, was mit der Vernachlässigung der Querschubverformungen und der Beschreibung der Kinematik des Schalenkontinuums im Sinne einer 3-Parameter-Schalentheorie einhergeht, so ist das Torsionsmoment auf dem Rand nicht unabhängig vorschreibbar. Folglich gilt die Momentenrandbedingung (2.25) nur für die Biegemomente und kann das o.g. Randintegral zum Potential beitragen. Im Weiteren wird das Potential (2.24) nur zur Berechnung schubstarrer Schalentragwerke verwendet. Allgemein differiert die Struktur des elastischen Potentials eines Systems in Abhängigkeit von dem Variationsprinzip und der Schalentheorie, während die kinetische Energie jeweils eine quadratische Form der Geschwindigkeiten ist, s. Gl. (2.7).

Zur Berechnung von Schalentragwerken mit der FEM ist die Lagrange-Funktion zu diskretisieren. Die räumliche Diskretisierung der relevanten Feldgrößen erfolgt mit bilinearen bzw. biquadratischen Ansätzen. Die Freiwerte der Ansätze und die arbeitsäquivalenten Knoteneinwirkungen sind für die Weggrößenformulierung mit

$$
\mathbf{v}_{k}^{T}=\left[\begin{array}{ll}
u_{i} & w_{\alpha}
\end{array}\right], \quad \mathbf{p}_{k}^{T}=\left[\begin{array}{lll}
\widehat{p}^{i} & 0 & 0
\end{array}\right],
$$

wobei $k$ den Knotenindex darstellt, und für die gemischte Formulierung mit

$$
\mathbf{z}_{k}^{T}=\left[\begin{array}{lll}
n^{\alpha \beta} & m^{\alpha \beta} & u_{i}
\end{array}\right], \quad \mathbf{p}_{k}^{T}=\left[\begin{array}{lll}
\widehat{\alpha}_{\alpha \beta} & \widehat{\beta}_{\alpha \beta} & \widehat{p}^{i}
\end{array}\right]
$$

gegeben. Imperfektionen sind mittels Variablensubstitution in den Modellgleichungen und eingeprägte Verzerrungen über den Belastungsvektor zu erfassen. Differentialgeometrische Größen werden analytisch bzw. mit den Ableitungen des diskretisierten Ortvektors $\mathbf{r}$ nach den konvektiven Koordinaten ermittelt. Auf die Transformation zwischen tensoriellen und physikalischen Feldgrößen sei hingewiesen. Nach der Diskretisierung und der Integration über die Elementgebiete folgt in der WeggröBenformulierung das elastische Potential zu

$$
\Pi(\mathbf{v})=\frac{1}{2} \mathbf{v}^{T} \mathbf{K}_{0} \mathbf{v}+\frac{1}{6} \mathbf{v}^{T} \mathbf{K}_{1}(\mathbf{v}) \mathbf{v}+\frac{1}{24} \mathbf{v}^{T} \mathbf{K}_{2}(\mathbf{v}, \mathbf{v}) \mathbf{v}-\mathbf{v}^{T} \mathbf{p} .
$$

Die Matrizen $\mathbf{K}_{0}, \mathbf{K}_{1}$ und $\mathbf{K}_{2}$ unterscheiden sich primär in den von ihnen erfassten geometrischen Nichtlinearitäten. Für die gemischte Formulierung wird das Potential zu

$$
\Pi(\mathbf{z})=\frac{1}{2} \mathbf{z}^{T} \mathbf{A}_{L} \mathbf{z}+\frac{1}{6} \mathbf{z}^{T} \mathbf{A}_{N L}(\mathbf{z}) \mathbf{z}-\mathbf{z}^{T} \mathbf{p}
$$

bestimmt. Die Matrix $\mathbf{A}_{L}$ repräsentiert die von den Zustandsgrößen quadratisch abhängigen Anteile des Potentials, die Matrix $\mathbf{A}_{N L}$ die kubisch abhängigen Anteile. Die erste Variation des Potentials (2.28) bzw. (2.29) charakterisiert Gleichgewichtszustände. Aus dem Hamilton-Prinzip (2.9) resultiert in der gemischten Formulierung nach Diskretisierung, Integration und Variation die semidiskrete Bewegungsgleichung

$$
\mathbf{M} \ddot{\mathbf{z}}+\mathbf{A}_{S}(\mathbf{z}) \mathbf{z}=\mathbf{p}, \text { mit } \mathbf{A}_{S}(\mathbf{z})=\mathbf{A}_{L}+\frac{1}{2} \mathbf{A}_{N L}(\mathbf{z}),
$$


deren Matrizen und Vektoren folgende Struktur aufweisen,

$$
\left[\begin{array}{ccc}
\mathbf{0} & \mathbf{0} & \mathbf{0} \\
\mathbf{0} & \mathbf{0} & \mathbf{0} \\
\mathbf{0} & \mathbf{0} & \mathbf{M}_{\ddot{i}}
\end{array}\right]\left[\begin{array}{l}
\mathbf{0} \\
\mathbf{0} \\
\ddot{\mathbf{u}}
\end{array}\right]+\left\{\left[\begin{array}{ccc}
-\mathbf{F}_{D} & \mathbf{0} & \mathbf{D} \\
\mathbf{0} & -\mathbf{F}_{B} & \mathbf{B} \\
\mathbf{D}^{T} & \mathbf{B}^{T} & \mathbf{K}_{B}
\end{array}\right]+\frac{1}{2}\left[\begin{array}{ccc}
\mathbf{0} & \mathbf{0} & \mathbf{G}_{v} \\
\mathbf{0} & \mathbf{0} & \mathbf{0} \\
\mathbf{G}_{v}^{T} & \mathbf{0} & \mathbf{G}_{s}
\end{array}\right]\right\}\left[\begin{array}{l}
\mathbf{n} \\
\mathbf{m} \\
\mathbf{u}
\end{array}\right]=\left[\begin{array}{c}
\widehat{\boldsymbol{\alpha}} \\
\widehat{\boldsymbol{\beta}} \\
\widehat{\mathbf{p}}
\end{array}\right] .
$$

Die ersten beiden Zeilen dieser Gleichung stellen die Verformungsbedingungen, die letzte Zeile stellt die Gleichgewichtsbedingungen dar. Mit der Matrix $\mathbf{K}_{B}$ kann eine linear elastische Bettung der Schalenmittelfläche berücksichtigt werden. Die Nachgiebigkeiten $\mathbf{F}_{D}$ und $\mathbf{F}_{B}$ sind unabhängig vom Systemzustand und positiv definit. Hinsichtlich der Elemente der in Gl. (2.31) angegebenen Untermatrizen sei auf [64] verwiesen. In den Vektoren $\mathbf{n}, \mathbf{m}$ und $\mathbf{u}$ sind die Freiwerte (2.27) und in den Vektoren $\widehat{\boldsymbol{\alpha}}, \widehat{\boldsymbol{\beta}}$ und $\widehat{\mathbf{p}}$ die arbeitsäquivalenten Knoteneinwirkungen (2.27) zusammengefasst. Die semidiskrete Bewegungsgleichung (2.30) wird mit dem Newmark-Verfahren gelöst. Auf die Kennzeichnung der Einwirkungen mit $\widehat{()}$ wird im Weiteren verzichtet.

\subsection{Integrales Plastizitätsmodell}

Die Bewertung des Tragverhaltens gedrungener Schalentragwerke aus Stahl erfordert die Berücksichtigung elastisch-plastischen Materialverhaltens. Hierzu sind Schichtenmodelle und integrale Plastizitätsmodelle geeignet. Der Berechnungsaufwand ist bei Schichtenmodellen signifikant von der Diskretisierung des Schalenkontinuums in Richtung der Schalennormalen abhängig und größer als bei integralen Plastizitätsmodellen. Daher wird im Rahmen dieser Arbeit das integrale Plastizitätsmodell nach Eggers/Kröplin [40] verwendet. Dieses Modell wird nachfolgend vorgestellt.

Für jeden Punkt des isotropen Schalenkontinuums ist die Fließbedingung

$$
Y^{*}=3 J_{2}-\sigma_{F}^{2}\left\{\begin{array}{l}
<0: \text { elastisches Materialverhalten } \\
=0: \text { plastisches Materialverhalten }
\end{array}\right.
$$

einzuhalten, wobei $J_{2}$ die zweite Invariante des Spannungsdeviators repräsentiert. Fließbedingung (2.32) folgt für zweidimensionale Spannungszustände zu

$$
Y^{*}=J_{\alpha \beta \rho \lambda} \sigma^{\alpha \beta} \sigma^{\rho \lambda}-\sigma_{F}^{2} \leq 0 \text {. }
$$

Die Komponenten des symmetrischen Transformationstensors $\mathbf{J}$ sind mit

$$
\begin{aligned}
& J_{1111}=J_{2222}=1,0, \quad J_{1122}=J_{2211}=-0,5, \\
& J_{1212}=J_{1221}=J_{2121}=J_{2112}=0,75
\end{aligned}
$$

gegeben. Die Spannungen im Schalenkontinuum sind mit Hilfe entsprechender Ansätze durch die Schnittgrößen beschreibbar. Für die Spannungen infolge der symmet- 
rischen und unsymmetrischen Membrankräfte wird ein über die Querschnittshöhe konstanter Verlauf angenommen. Für die Spannungen aus den Momenten ist bei nicht plastifiziertem Querschnitt ein linearer Verlauf und bei vollständig plastifiziertem Querschnitt ein bikonstanter Verlauf über die Querschnittshöhe vorhanden. Die entsprechend gewählten Ansätze erfüllen bestimmte Orthogonalitätsbedingungen, um die eindeutige Zuordnung von Spannungen und Schnittgrößen zu gewährleisten.

Mit den Ansätzen für den Verlauf der Spannungen ist aus Fließbedingung (2.33) die Bedingung für den elastischen Grenzzustand,

$$
Y_{0}=Y_{0}\left(n^{\alpha \beta}, m^{\alpha \beta}\right)=\widetilde{A}_{11}+3\left|\widetilde{A}_{12}\right|+2,25 A_{22}-S_{0}^{2}=0,
$$

ermittelbar. Entsprechend folgt die Bedingung für den plastischen Grenzzustand zu

$$
Y_{1}=Y_{1}\left(n^{\alpha \beta}, m^{\alpha \beta}\right)=\widetilde{A}_{11}+\frac{1}{2} A_{22}+\sqrt{\left(\widetilde{A}_{12}\right)^{2}+\left(\frac{1}{2} A_{22}\right)^{2}}-S_{1}^{2}=0,
$$

mit $S_{1}=S_{0}=\sigma_{F} t$ und den Termen

$$
\begin{aligned}
\widetilde{A}_{11} & =A_{11}-A_{11}^{b}+A_{11}^{b b}, & \widetilde{A}_{12} & =A_{12}-A_{12}^{b}, \\
A_{11} & =J_{\alpha \beta \gamma \lambda} n^{\alpha \beta} n^{\gamma \lambda}, & A_{11}^{b} & =2 J_{\alpha \beta \gamma \lambda} n^{\alpha \beta} b_{\mu}^{\lambda} m^{\gamma \mu}, \\
A_{11}^{b b} & =J_{\alpha \beta \gamma \lambda} b_{\rho}^{\beta} b_{\mu}^{\lambda} m^{\alpha \rho} m^{\gamma \mu}, & A_{12} & =\frac{4}{t} J_{\alpha \beta \gamma \lambda} n^{\alpha \beta} m^{\gamma \lambda}, \\
A_{12}^{b} & =\frac{4}{t} J_{\alpha \beta \gamma \lambda} b_{\rho}^{\beta} m^{\alpha \rho} m^{\gamma \lambda}, & A_{22} & =\frac{16}{t^{2}} J_{\alpha \beta \gamma \lambda} m^{\alpha \beta} m^{\gamma \lambda} .
\end{aligned}
$$

Für $Y_{0}<0$ ist der Querschnitt nicht plastifiziert, für $Y_{1}=0$ vollständig plastifiziert. Für $Y_{0} \geq 0, Y_{1}<0$ ist der Querschnitt teilweise plastifiziert. Mit der Fließbedingung

$$
Y=Y\left(n^{\alpha \beta}, m^{\alpha \beta}, \varepsilon\right)=\frac{b Y_{0}+\varepsilon^{a} Y_{1}}{b+\varepsilon^{a}}=0
$$

ist die Plastifizierung des Schalenkontinuums in Richtung der Schalennormalen beschreibbar. Die Parameter $a$ und $b$ folgen aus einer Vergleichsberechnung mit Hilfe eines Schichtenmodells zu $a=0,90$ und $b=1 / 15$. Der Parameter

$$
\varepsilon=\varepsilon_{V}^{p} / \varepsilon_{V}^{e}=\varepsilon_{V}^{p} E t / S_{V}
$$

ermöglicht die Beschreibung der Plastifizierung des Schalenkontinuums mit Größen eines fiktiven eindimensionalen Vergleichszustandes. Die Vergleichskraft $S_{V}$, die plastische Vergleichsverzerrung $\varepsilon_{V}^{p}$ und der Parameter $\varepsilon$ sind von der Belastungsgeschichte abhängig. Bild 2.3 verdeutlicht die Entwicklung der Schnittgrößen, der Vergleichskraft und des Parameters $\varepsilon$ während des Plastifizierens eines Querschnittes.

Für den nicht plastifizierten Querschnitt ist der Parameter $\varepsilon$ gleich null. Für den plastifizierten Querschnitt ist die Entwicklung des Parameters von der Art der Beanspruchung abhängig. Bei Membrankraftbeanspruchung beschreibt die Fließbedingung einen $C_{0}$-stetigen Übergang zwischen elastischem und plastischem Grenzzustand. 

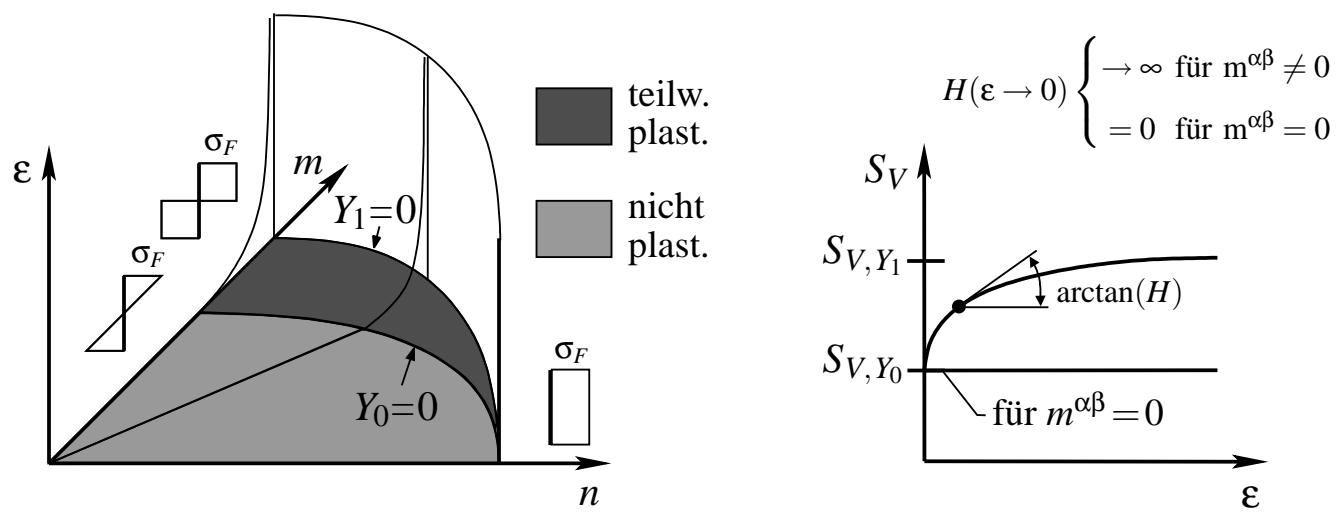

Bild 2.3: Plastifizieren eines Querschnittes vs. Vergleichszustand

Bei Momentenbeanspruchung weist der Übergang $C_{1}$-Stetigkeit auf. Mit Annäherung an den plastischen Grenzzustand strebt der Parameter $\varepsilon$ gegen unendlich. Ähnliche Zusammenhänge bestehen zwischen der Vergleichskraft und dem Parameter $\varepsilon$.

Bei der Berechnung der Plastifizierung des Schalenkontinuums ist zu unterscheiden, ob im aktuellen Inkrement ein Durchstoßen der Fließfläche $Y_{0}$ erfolgt oder der zuletzt ermittelte Spannungszustand bereits die Fließbedingung (2.36) erfüllt. Beim Durchstoßen der Fließfläche ist von den inkrementellen Zustandsgrößen $\Delta \mathbf{z}$ der elastisches Materialverhalten repräsentierende Anteil $\eta \Delta \mathbf{z}$ zu ermitteln. Der Parameter $\eta$ folgt aus der Bedingung (2.34) und ist bei Systemen mit großer Anzahl von Freiwerten zur Verringerung des Berechnungsaufwandes um das Toleranzmaß $\varepsilon_{\eta}$ zu erweitern [87]. Mit dem Anteil $\eta \Delta \mathbf{z}$ sind die Zustandsgrößen an den entsprechenden Knoten bei Erreichen der Fließfläche beschreibbar. Der verbleibende Anteil des Inkrementes wird unter Berücksichtigung des nunmehr zumindest für einige Knoten vorhandenen plastischen Materialverhaltens iterativ und wie nachfolgend aufgezeigt berechnet.

Nach Erreichen des elastischen Grenzzustandes ist für die entsprechenden Knoten bei Laststeigerung die Fließbedingung (2.36) einzuhalten. Diese Forderung ist mit Hilfe der Konsistenzbedingung zu erfüllen. Für eine inkrementelle Änderung der Zustandsgrößen folgt die mit $1 /\left(2 S_{V}\right)$ skalierte Konsistenzbedingung zu

$$
\frac{\Delta Y}{2 S_{V}}=\int_{\mathbf{z}}^{\mathbf{z}+\Delta \mathbf{z}} \frac{d Y}{2 S_{V}} \approx v_{\alpha \beta}^{*} \Delta n^{\alpha \beta}+\mu_{\alpha \beta}^{*} \Delta m^{\alpha \beta}-\bar{H}^{*} \Delta \varepsilon_{V}^{p}=0,
$$

mit den Termen

$$
v_{\alpha \beta}^{*}=\frac{1}{2}\left(\left.\frac{\partial Y}{2 S_{V} \partial n^{\alpha \beta}}\right|_{\mathbf{z}}+\left.\frac{\partial Y}{2 S_{V} \partial n^{\alpha \beta}}\right|_{\mathbf{z}+\Delta \mathbf{z}}\right),
$$

entsprechend definierten Termen $\mu_{\alpha \beta}^{*}$ sowie

$$
\bar{H}^{*}=-\frac{1}{2}\left(\left.\frac{\partial Y}{2 S_{V} \partial \varepsilon_{V}^{p}}\right|_{\mathbf{z}}+\left.\frac{\partial Y}{2 S_{V} \partial \varepsilon_{V}^{p}}\right|_{\mathbf{z}+\Delta \mathbf{z}}\right) .
$$


Zur Beschreibung elastisch-plastischen Materialverhaltens ist neben der Konsistenzbedingung auch eine Fließregel erforderlich. Die Fließregel definiert die Entwicklung der plastischen Verzerrungen. Bei Anwendung der assoziierten Fließregel

$$
d \alpha_{\alpha \beta}^{p}=\frac{\partial Y}{\partial n^{\alpha \beta}} d \lambda \quad \text { bzw. } \quad d \beta_{\alpha \beta}^{p}=\frac{\partial Y}{\partial m^{\alpha \beta}} d \lambda
$$

ist die Richtung des differentiellen Zuwachses der plastischen Verzerrungen mit den Ableitungen der Fließbedingung nach den Schnittgrößen festgelegt. Der differentielle Zuwachs des Parameters $\lambda$ wird anhand des Vergleiches der differentiellen Dissipationsarbeit im Schalenkontinuum mit der des Vergleichszustandes zu $d \varepsilon_{V}^{p} /\left(2 S_{V}\right)$ bestimmt. Die inkrementellen plastischen Verzerrungen

$$
\Delta \alpha_{\alpha \beta}^{p} \approx v_{\alpha \beta}^{*} \Delta \varepsilon_{V}^{p} \text { bzw. } \Delta \beta_{\alpha \beta}^{p} \approx \mu_{\alpha \beta}^{*} \Delta \varepsilon_{V}^{p}
$$

sind unter Annahme kleiner Verzerrungen und einer additiven Zerlegung der Gesamtverzerrungen in den Verformungsbedingungen zu berücksichtigen. Zur Bewertung des Tragverhaltens von Schalentragwerken bei elastisch-plastischem Materialverhalten wird das inkrementelle elastische Potential (2.54) formal erweitert,

$$
\Delta \Pi=\Delta \Pi_{(2.54)}-\int_{A} \Delta \varepsilon_{V}^{p}\left\{\boldsymbol{v}^{T} \Delta \mathbf{n}+\boldsymbol{\mu}^{T} \Delta \mathbf{m}-\frac{1}{2} \bar{H}^{*} \Delta \varepsilon_{V}^{p}\right\} d A,
$$

wobei die Vektoren $\boldsymbol{v}$ und $\boldsymbol{\mu}$ die Terme $v_{\alpha \beta}^{*}$ und $\mu_{\alpha \beta}^{*}$ beinhalten. Die Diskretisierung der plastischen Vergleichsverzerrung erfolgt mit bilinearen bzw. biquadratischen Ansätzen; entsprechend wird der Vektor $\mathbf{z}$ um die Freiwerte der Ansätze für die plastische Vergleichsverzerrung ergänzt. Auf Knotenpunktebene führt die erste Variation des zweiten Anteiles des erweiterten Potentials (2.43) auf die Sekantenmatrix $\tilde{\mathbf{A}}_{S, P}$ und die Linearisierung dieser Variation auf die Tangentenmatrix $\tilde{\mathbf{A}}_{T, P}$,

$$
\tilde{\mathbf{A}}_{S, P}=-\left[\begin{array}{cccc}
\mathbf{0} & \mathbf{0} & \mathbf{0} & \mathbf{v} \\
\mathbf{0} & \mathbf{0} & \mathbf{0} & \boldsymbol{\mu} \\
\mathbf{0} & \mathbf{0} & \mathbf{0} & \mathbf{0} \\
\mathbf{v}^{T} & \boldsymbol{\mu}^{T} & \mathbf{0} & -\bar{H}^{*}
\end{array}\right], \quad \tilde{\mathbf{A}}_{T, P}=-\left[\begin{array}{cccc}
\mathbf{T}_{11} & \mathbf{T}_{12} & \mathbf{0} & \mathbf{T}_{14} \\
\mathbf{T}_{21} & \mathbf{T}_{22} & \mathbf{0} & \mathbf{T}_{24} \\
\mathbf{0} & \mathbf{0} & \mathbf{0} & \mathbf{0} \\
\mathbf{T}_{41} & \mathbf{T}_{42} & \mathbf{0} & \mathbf{T}_{44}
\end{array}\right] .
$$

Die ersten beiden Zeilen der Matrizen repräsentieren die in die Verformungsbedingungen eingehenden plastischen Verzerrungen bzw. deren linearisierte Form, die letzte Zeile die Konsistenzbedingung bzw. deren linearisierte Form. Hinsichtlich der Elemente der unsymmetrischen Tangentenmatrix, die im Rahmen dieser Arbeit symmetrisiert wird, sei auf $[139,87]$ verwiesen. Die Sekanten- und die Tangentenmatrix (2.44) sind für die entsprechenden Knoten zu berechnen. Zur Integration der Konsistenzbedingung und der Fließregel über das Elementgebiet sind die Elemente der Sekanten- und der Tangentenmatrix auf die Gaußpunkte abzubilden. Aus der Integration resultieren auf Systemebene die Matrizen $\mathbf{A}_{S, P}(\mathbf{z}, \Delta \mathbf{z})$ und $\mathbf{A}_{T, P}(\mathbf{z}, \Delta \mathbf{z})$. 
Während der iterativen Berechnung eines neuen Systemzustandes mit dem NewtonRaphson-Verfahren sind fortlaufend für die entsprechenden Knoten das Inkrement der mit $S_{V}=\sigma_{F} t$ initialisierten Vergleichskraft mit

$$
\Delta S_{V}=\bar{H}^{*} \Delta \varepsilon_{V}^{p}=H^{*} \Delta \varepsilon
$$

sowie die Tangentenmatrix $\mathbf{A}_{T, P}(\mathbf{z}, \Delta \mathbf{z})$ und zur Berechnung des Residuums die Sekantenmatrix $\mathbf{A}_{S, P}(\mathbf{z}, \Delta \mathbf{z})$ zu aktualisieren. Nach der Ermittlung eines neuen Systemzustandes ist die von den inkrementellen Zustandsgrößen geleistete Dissipationsarbeit

$$
\Delta D=\int_{A} \Delta \varepsilon_{V}^{p}\left\{v_{\alpha \beta}^{*} \Delta n^{\alpha \beta}+\mu_{\alpha \beta}^{*} \Delta m^{\alpha \beta}\right\} d A=-\Delta \mathbf{z}^{T} \mathbf{A}_{S, P}(\mathbf{z}, \Delta \mathbf{z}) \Delta \mathbf{z}
$$

berechenbar. Den Hauptsätzen der Thermodynamik zufolge ist die Dissipationsarbeit stets positiv. Alternativ zur integralen Formulierung können die Konsistenzbedingung und die Fließregel diskret für die entsprechenden Knoten ausgewertet werden.

Eigenschaften des integralen Plastizitätsmodells werden unter Annahme geometrisch linearen Tragverhaltens für das in Bild 2.4 dargestellte Stabtragwerk diskutiert. Zur
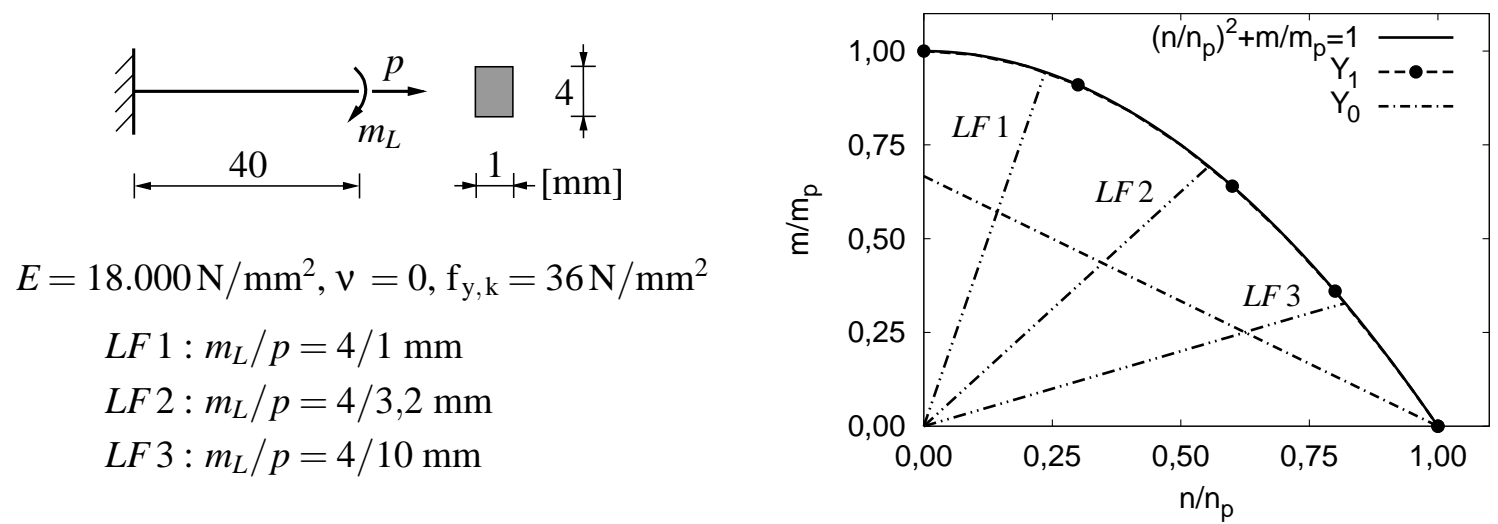

Bild 2.4: Stabtragwerk - Vergleich von numerischer und analytischer Lösung

Diskretisierung des erweiterten Potentials (2.43) werden vier gemischte Elemente mit biquadratischen Ansätzen und gleicher Größe verwendet. Für drei Lastfälle und einen Knoten an der Einspannung verdeutlicht das Diagramm die Entwicklung der Fließbedingung (2.36) in Abhängigkeit vom Lastniveau. Bei Erreichen des elastischen Grenzzustandes beschreibt die Fließbedingung einen linearen Zusammenhang zwischen Biegemoment $m$ und Normalkraft $n$. Dieser Zusammenhang wird bei weiterer Laststeigerung aufgrund des unterschiedlichen Einflusses der Schnittgrößen auf die Plastifizierung des Stabtragwerkes zunehmend nichtlinear. Für den plastischen Grenzzustand stimmen die numerische und die analytische Lösung gut überein.

Wird die Belastung so verändert, dass sich zusätzlich zur Normalkraft ein linearer Verlauf des Biegemomentes einstellt, treten Oszillationen im Verlauf der Normalkraft auf. Die Oszillationen sind durch die gleichzeitige Diskretisierung der Normalkraft, des 
Biegemomentes und der plastischen Vergleichsverzerrung mit biquadratischen bzw. bilinearen Ansätzen bedingt. Die Oszillationen verschwinden jedoch mit feinerer Diskretisierung des plastifizierten Bereiches des Stabtragwerkes. Ein weiterer Grund für eine feine Diskretisierung wird bei Vernachlässigung der Horizontallast $p$ deutlich. Mit zunehmender Plastifizierung des Tragwerkes in Stablängsrichtung erreichen weitere Knoten den elastischen Grenzzustand. Für diese Knoten sind Elemente der Sekanten- und der Tangentenmatrix zu modifizieren. Insbesondere der Term $H$, in inkrementeller Formulierung $H^{*}$, kann, wie Bild 2.3 rechts erkennen lässt, bei zu grober Diskretisierung des erweiterten Potentials eine signifikante Änderung der Systemantwort bewirken. Oszillationen während der Lösung der Randwertaufgabe treten bei kombinierter Normalkraft- und Momentenbeanspruchung auf, wenn eine der Beanspruchungen gegen null tendiert, da in diesem Fall die Fließbedingung (2.36) nicht $C_{1}$-stetig ist. Mittels Deaktivierung des Kopplungsterms $A_{12}$ an den entsprechenden Knoten ist $C_{1}$-Stetigkeit zu erreichen, sodass keine Oszillationen auftreten. Die damit einhergehende lokale Änderung der Konsistenzbedingung und der Fließregel beeinflusst die Güte der berechneten Lösung auf Systemebene nur geringfügig [124].

Die Anwendung des integralen Plastizitätsmodells ist auf Fälle mit kleinen Verzerrungen und geringem Einfluss der Querkräfte auf die Plastifizierung des Schalenkontinuums beschränkt. Ferner ist bei großen Flächenkrümmungen die Güte des berechneten elastischen Grenzzustandes davon abhängig, wie genau die gewählten Ansätze den tatsächlichen Verlauf der Spannungen im Schalenkontinuum approximieren.

\subsection{Faserverbundwerkstoffe}

Schalentragwerke können z. B. aus Faserverbundwerkstoff gefertigt werden, wenn eine Gewichtsoptimierung erforderlich ist. Faserverbundwerkstoffe sind spezielle Verbundwerkstoffe und besitzen geringes Gewicht, große Festigkeit und hohe Widerstandsfähigkeit gegenüber Korrosion. Ferner sind Schalentragwerke aus Faserverbundwerkstoff relativ einfach den konstruktiven Anforderungen anzupassen.

Faserverbundwerkstoffe sind in der Regel anisotrop und bestehen aus Einzelschichten mit oft unterschiedlichen mechanischen Eigenschaften, vgl. Bild 2.5. Der Verbund zwischen den Einzelschichten ist von der konstruktiven Durchbildung abhängig. Eine Einzelschicht besteht aus Fasern und Matrix. Fasermaterialien sind Kohlenstoff und Glas. Die Matrix kann aus Epoxid- oder Polyesterharz hergestellt werden. Die Steifigkeit einer Einzelschicht ist auch von der Anordnung der Fasern abhängig. Bei unidirektionaler Anordnung der Fasern spricht man von einer UD-Schicht.

Die Modellierung eines Faserverbundwerkstoffes erfordert die Beschreibung des Tragverhaltens der Einzelschichten. Für UD-Schichten ist im Rahmen dieser Arbeit linear 

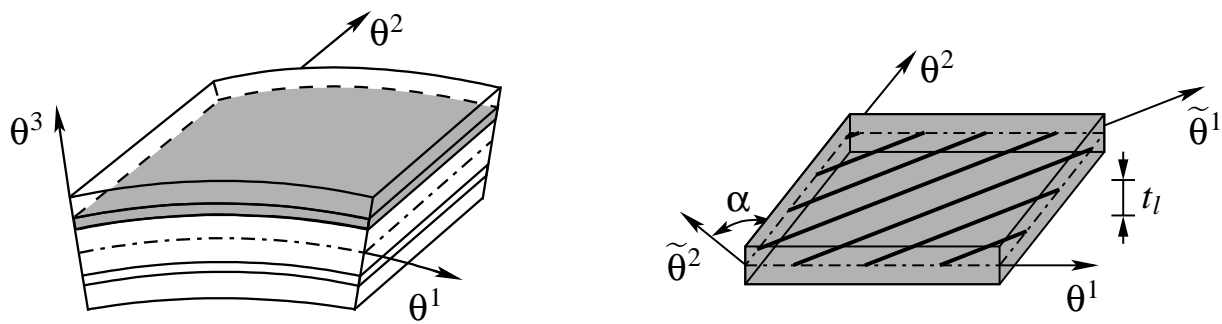

Bild 2.5: Aufbau eines Faserverbundwerkstoffes und einer UD-Schicht

elastisches, orthotropes Materialverhalten anzunehmen. Ferner sind thermische und hygroskopische Einflüsse vernachlässigbar. Bei Annahme eines zweidimensionalen Spannungszustandes folgen im orthonormierten Materialhauptachsensystem $\widetilde{\theta}^{\alpha}$, vgl. Bild 2.5 rechts, die konstitutiven Gleichungen für eine UD-Schicht zu

$$
\widetilde{S}^{\alpha \beta}=\widetilde{C}^{\alpha \beta \gamma \rho} \widetilde{E}_{\gamma \rho},
$$

mit den Parametern

$$
\widetilde{C}^{1111}=\frac{\widetilde{E}_{1}}{1-\widetilde{v}_{12}^{2} \widetilde{E}_{2} / \widetilde{E}_{1}}, \widetilde{C}^{2222}=\frac{\widetilde{E}_{2}}{1-\widetilde{v}_{12}^{2} \widetilde{E}_{2} / \widetilde{E}_{1}}, \widetilde{C}^{1122}=\widetilde{C}^{2211}=\widetilde{v}_{12} \widetilde{C}^{2222}
$$

und $\widetilde{C}^{1212}=\widetilde{C}^{1221}=\widetilde{C}^{2112}=\widetilde{C}^{2121}=\widetilde{G}_{12}$. Die Parameter $\widetilde{C}^{\alpha \beta \gamma \rho}$ sind mit vier unabhängigen effektiven Materialparametern beschreibbar [3]. Allgemein ist die Steifigkeit einer UD-Schicht in Richtung der Materialhauptachse $\widetilde{\theta}^{1}$ deutlich größer als in Richtung der zweiten Materialhauptachse. Zur Berechnung der konstitutiven Gleichungen für einen Faserverbundwerkstoff ist Gl. (2.47) mit

$$
S^{\alpha \beta}=\frac{\partial \theta^{\alpha}}{\partial \widetilde{\theta}^{\lambda}} \frac{\partial \theta^{\beta}}{\partial \widetilde{\theta}^{\mu}} \widetilde{S}^{\lambda \mu} \quad \text { und } \quad E_{\gamma \rho}=\frac{\partial \widetilde{\theta}^{\lambda}}{\partial \theta^{\gamma}} \frac{\partial \widetilde{\theta}^{\mu}}{\partial \theta^{\rho}} \widetilde{E}_{\lambda \mu}
$$

in das Koordinatensystem $\theta^{\alpha} \mathrm{zu}$ transformieren,

$$
S^{\alpha \beta}=C^{\alpha \beta \gamma \rho} E_{\gamma \rho} .
$$

Die Transformationen (2.48) verdeutlichen die für anisotropes Materialverhalten oft charakteristische Kopplung sämtlicher Spannungen und Verzerrungen.

Zur Beschreibung des Zusammenwirkens der Einzelschichten im Faserverbundwerkstoff sind Annahmen zur Kinematik erforderlich. Die klassische Laminattheorie setzt idealen Verbund zwischen den Einzelschichten und die Gültigkeit der KirchhoffLove-Hypothese voraus. Werden die Spannungen und deren statisches Moment bezüglich der Schalenmittelfläche über die $n$ Schichten des Faserverbundwerkstoffes summiert, so folgt unter Berücksichtigung der Verzerrungen (2.14)

$$
\left[\begin{array}{c}
n^{\alpha \beta} \\
m^{\alpha \beta}
\end{array}\right]=\left[\begin{array}{ll}
C_{D}^{\alpha \beta \gamma \rho} & C_{C}^{\alpha \beta \gamma \rho} \\
C_{C}^{\alpha \beta \gamma \rho} & C_{B}^{\alpha \beta \gamma \rho}
\end{array}\right]\left[\begin{array}{l}
\alpha_{\gamma \rho} \\
\beta_{\gamma \rho}
\end{array}\right], \text { mit } C_{D}^{\alpha \beta \gamma \rho}=\sum_{k=1}^{n} t_{l}^{k} C_{k}^{\alpha \beta \gamma \rho}
$$


sowie

$$
C_{C}^{\alpha \beta \gamma \rho}=\frac{1}{2} \sum_{k=1}^{n}\left(\left(\theta_{k}^{3}\right)^{2}-\left(\theta_{k-1}^{3}\right)^{2}\right) C_{k}^{\alpha \beta \gamma \rho}, C_{B}^{\alpha \beta \gamma \rho}=\frac{1}{3} \sum_{k=1}^{n}\left(\left(\theta_{k}^{3}\right)^{3}-\left(\theta_{k-1}^{3}\right)^{3}\right) C_{k}^{\alpha \beta \gamma \rho} .
$$

Die Parameter $C_{D}^{\alpha \beta \gamma \rho}, C_{C}^{\alpha \beta \gamma \rho}$ und $C_{B}^{\alpha \beta \gamma \rho}$ sind vom Aufbau des Faserverbundwerkstoffes abhängig. Bei zur Schalenmittelfläche symmetrischen Faserverbundwerkstoffen sind die Parameter $C_{C}^{\alpha \beta \rho}$ identisch null. Folglich sind Membran- und Biegetragwirkung entkoppelt. Für die gemischte Formulierung sind die konstitutiven Gleichungen (2.50) in den Verformungsbedingungen zu berücksichtigen. Nach der Diskretisierung der Feldgrößen und der Integration über die Elementgebiete folgt die Matrix $\mathbf{A}_{L} \mathbf{z u}$

$$
\mathbf{A}_{L}=\left[\begin{array}{ccc}
-\mathbf{F}_{D}^{*} & -\mathbf{F}_{C}^{*} & \mathbf{D} \\
-\mathbf{F}_{C}^{* T} & -\mathbf{F}_{B}^{*} & \mathbf{B} \\
\mathbf{D}^{T} & \mathbf{B}^{T} & \mathbf{0}
\end{array}\right]
$$

vgl. Gl. (2.31). Der Aufwand zur Berechnung der Matrix ist im Vergleich zum Fall isotropen Materialverhaltens nur geringfügig größer. Randbedingungen sind weiterhin mit den Zustandsgrößen der Schalenmittelfläche zu berücksichtigen.

Zur Bewertung der Beanspruchung eines Faserverbundwerkstoffes sind die Konzepte vom Versagen der ersten bzw. der letzten Schicht geeignet [3]. Die Konzepte basieren auf unterschiedlichen Sicherheitsphilosophien und erfordern Kriterien zur Bewertung der Beanspruchung der Einzelschichten. Mit den Kriterien sind unterschiedliche Versagensformen einer Einzelschicht zu erfassen. Derartige Versagensformen sind der Faser- und der Zwischenfaserbruch. Im Rahmen dieser Arbeit wird das Konzept vom Versagen der ersten Schicht unter Berücksichtigung des Maximumspannungskriteriums verwendet. Die hierbei anzusetzenden Grenzspannungen sind vom Vorzeichen der Spannungen in der jeweiligen UD-Schicht abhängig.

Die Verifikation der Implementierung erfolgt am Beispiel zweier Zugproben. Beide Zugproben besitzen die in Bild 2.6 dargestellte Geometrie und werden mit $p=$ $100 \mathrm{~N} / \mathrm{mm}$ belastet. Der Faserverbundwerkstoff der Zugproben besteht aus zwei UD-Schichten mit gleicher Dicke und den Materialparametern $\widetilde{E}_{1}=31.100 \mathrm{~N} / \mathrm{mm}^{2}$, $\widetilde{E}_{2}=7.600 \mathrm{~N} / \mathrm{mm}^{2}, \widetilde{v}_{12}=0,303$ und $\widetilde{G}_{12}=2.900 \mathrm{~N} / \mathrm{mm}^{2}$. Für Zugprobe Z1 ist die Orientierung der Fasern mit ${ }_{u}[90,0]$ und für Zugprobe Z2 mit $[-45,45]$ festgelegt.

Zur Diskretisierung des elastischen Potentials werden gemischte Elemente mit bilinearen Ansätzen und gleicher Geometrie verwendet. Die Abbildung der realen Randbedingungen, d.h. der Klemmbacken der Prüfmaschine, erfordert zusätzlich zur angegebenen Einspannung die Nullsetzung der Längsneigung am belasteten Rand sowie die Annahme sehr steifen isotropen Materialverhaltens mit $E=1,0 \cdot 10^{10} \mathrm{~N} / \mathrm{mm}^{2}$ 

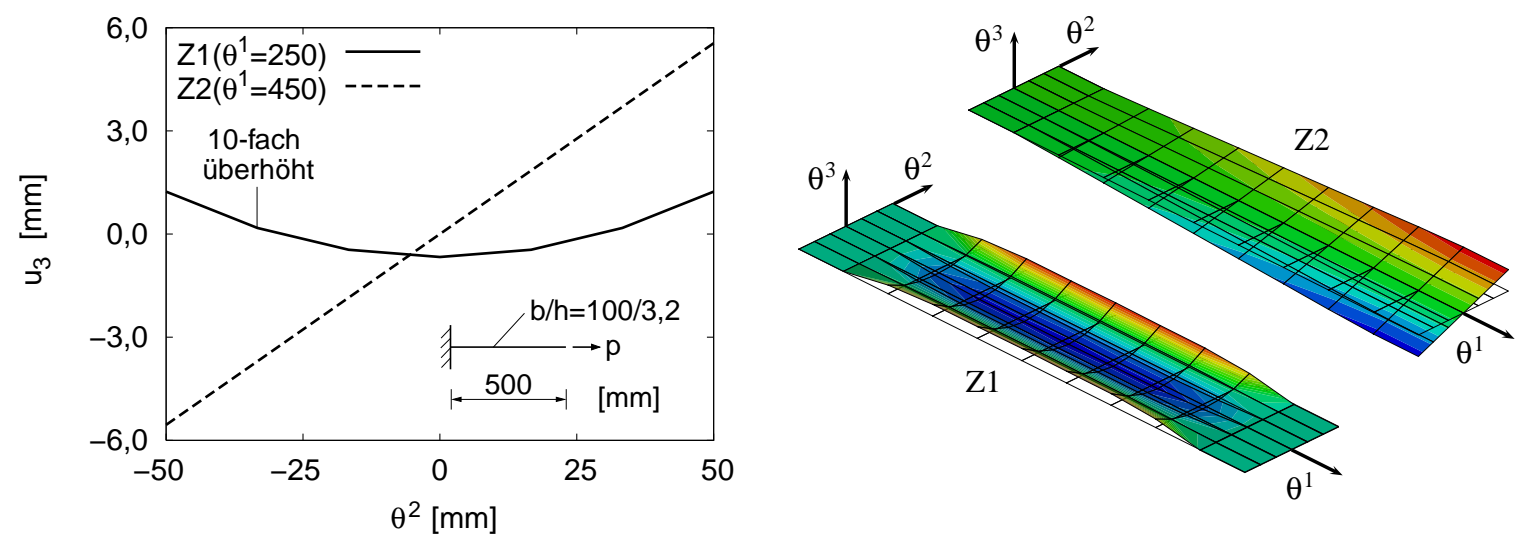

Bild 2.6: Verformungen der zweischichtigen Zugproben Z1 und Z2

und $v=0$ für die erste und letzte Elementreihe in Längsrichtung. Die Berechnung berücksichtigt das geometrisch nichtlineare Tragverhalten der Zugproben. Die Ergebnisse stimmen gut mit der Referenzlösung $[55,126]$ überein und zeigen die Abhängigkeit des Verformungsverhaltens vom Aufbau des Faserverbundwerkstoffes auf. Während Zugprobe Z1 durch eine Zug-Biege-Kopplung charakterisiert ist, tritt für Zugprobe Z2 eine Zug-Torsions-Kopplung auf. Ferner wird deutlich, dass bei Schalentragwerken aus Faserverbundwerkstoff Störungen des Membranspannungszustandes außerhalb der Randbereiche bereits bei linearem Tragverhalten möglich sind.

\subsection{Lösung diskretisierter Anfangs-Randwertaufgaben}

Die Beschreibung des Tragverhaltens von Schalentragwerken erfolgt im Rahmen dieser Arbeit primär auf Grundlage der Lagrange-Funktion und des inkrementellen elastischen Potentials. Die hierbei auftretenden Gleichungssysteme können ihren mathematischen Eigenschaften entsprechend mit verschiedenen Verfahren gelöst werden.

\subsubsection{Inkrementelle Formulierung}

Zur Lösung der bei statischen Systemen gegebenen Randwertaufgabe ist in ausgewählten Fällen die inkrementelle Formulierung des elastischen Potentials erforderlich. Diese Formulierung ist mit

$$
\Delta \Pi=\Pi_{1}-\Pi_{0}=\delta \Pi_{0}+\frac{1}{2} \delta^{2} \Pi_{0}+\frac{1}{6} \delta^{3} \Pi_{0}+\frac{1}{24} \delta^{4} \Pi_{0}+\ldots
$$

zu berechnen, wobei im Ausgangszustand 0 die erste Variation des Potentials identisch null ist. Für die Weggrößenformulierung folgt das inkrementelle Potential zu

$$
\begin{aligned}
\Delta \Pi\left(\mathbf{v}_{0}, \Delta \mathbf{v}\right) & =\frac{1}{2} \Delta \mathbf{v}^{T} \mathbf{K}_{0}\left(\mathbf{v}_{0}, \mathbf{v}_{0}\right) \Delta \mathbf{v}+\frac{1}{6} \Delta \mathbf{v}^{T} \mathbf{K}_{1}\left(\mathbf{v}_{0}, \Delta \mathbf{v}\right) \Delta \mathbf{v} \\
& +\frac{1}{24} \Delta \mathbf{v}^{T} \mathbf{K}_{2}(\Delta \mathbf{v}, \Delta \mathbf{v}) \Delta \mathbf{v}-\Delta \mathbf{v}^{T} \Delta \mathbf{p}
\end{aligned}
$$


Die Matrizen $\mathbf{K}_{0}, \mathbf{K}_{1}$ und $\mathbf{K}_{2}$ differieren primär in den Zustandsgrößen, die in die Matrizen eingehen, und berücksichtigen unterschiedliche geometrische Nichtlinearitäten. In der gemischten Formulierung wird das inkrementelle Potential zu

$$
\Delta \Pi\left(\mathbf{z}_{0}, \Delta \mathbf{z}\right)=\frac{1}{2} \Delta \mathbf{z}^{T} \mathbf{A}_{T}\left(\mathbf{z}_{0}\right) \Delta \mathbf{z}+\frac{1}{6} \Delta \mathbf{z}^{T} \mathbf{A}_{N L}(\Delta \mathbf{z}) \Delta \mathbf{z}-\Delta \mathbf{z}^{T} \Delta \mathbf{p}
$$

bestimmt. Dabei ist im Gegensatz zur Weggrößenformulierung die vierte Variation des elastischen Potentials identisch null. Die Matrix $\mathbf{A}_{T}\left(\mathbf{z}_{0}\right)=\mathbf{A}_{L}+\mathbf{A}_{N L}\left(\mathbf{z}_{0}\right)$ repräsentiert die Tangentenmatrix im Ausgangszustand, die Matrix $\mathbf{A}_{N L}(\Delta \mathbf{z})$ weitere nichtlineare Anteile im Inkrement. Die erste Variation des inkrementellen Potentials (2.53) nach den inkrementellen Zustandsgrößen führt auf

$$
\begin{aligned}
\delta \Delta \Pi\left(\mathbf{v}_{0}, \Delta \mathbf{v}\right) & =\delta \Delta \mathbf{v}^{T}\left[\mathbf{K}_{0}\left(\mathbf{v}_{0}, \mathbf{v}_{0}\right)+\frac{1}{2} \mathbf{K}_{1}\left(\mathbf{v}_{0}, \Delta \mathbf{v}\right)+\frac{1}{6} \mathbf{K}_{2}(\Delta \mathbf{v}, \Delta \mathbf{v})\right] \Delta \mathbf{v} \\
& -\delta \Delta \mathbf{v}^{T} \Delta \mathbf{p}=0
\end{aligned}
$$

und die entsprechende Variation des inkrementellen Potentials (2.54) auf

$$
\delta \Delta \Pi\left(\mathbf{z}_{0}, \Delta \mathbf{z}\right)=\delta \Delta \mathbf{z}^{T}\left\{\left[\mathbf{A}_{T}\left(\mathbf{z}_{0}\right)+\frac{1}{2} \mathbf{A}_{N L}(\Delta \mathbf{z})\right] \Delta \mathbf{z}-\Delta \mathbf{p}\right\}=0 .
$$

Beide Variationen charakterisieren Gleichgewichtszustände. Dabei stellen die Terme in den eckigen Klammern die Sekantenmatrix $\mathbf{K}_{s}\left(\mathbf{v}_{0}, \Delta \mathbf{v}\right)$ bzw. $\mathbf{A}_{S}\left(\mathbf{z}_{0}, \Delta \mathbf{z}\right)$ dar, wobei letztere Matrix bei elastisch-plastischem Materialverhalten um die in Abschnitt 2.3 hergeleitete Sekantenmatrix $\mathbf{A}_{S, P}\left(\mathbf{z}_{0}, \Delta \mathbf{z}\right)$ ergänzt wird.

\subsubsection{Inhomogene Gleichungssysteme}

Zur Lösung der in der ersten Variation der Potentiale (2.28-2.29) und (2.53-2.54) enthaltenen inhomogenen Gleichungssysteme wird im Rahmen dieser Arbeit primär das Newton-Raphson-Verfahren verwendet. Das Verfahren erfordert die Linearisierung der Gleichungssysteme. Aus der Linearisierung resultieren in Abhängigkeit von der FE-Formulierung die Tangentenmatrizen $\mathbf{K}_{T}(\mathbf{v}, \mathbf{v})$ und $\mathbf{A}_{T}(\mathbf{z})$. Das Newton-RaphsonVerfahren ermöglicht lokal quadratisches Konvergenzverhalten, kann aber im Bereich von Extrema des Last-Verformungs-Pfades versagen. Diesem Nachteil ist mit der quasi-viskosen Dämpfung und dem Bogenlängenverfahren zu begegnen.

Die quasi-viskose Dämpfung [95] basiert auf der Berücksichtigung von Dämpfungstermen in den Gleichgewichtsbedingungen. Die Terme sind von der Größe des Residuums abhängig und infolge der Zeitinvarianz statischer Prozesse als zusätzliche Steifigkeiten in der Tangentenmatrix interpretierbar. Die quasi-viskose Dämpfung ermöglicht, ausgehend von einem stabilen Gleichgewichtszustand im Bereich eines Extremums und über instabile Bereiche des Last-Verformungs-Pfades hinweg, die 
Ermittlung stabiler Gleichgewichtszustände. Eine etwaige Verringerung der Konvergenzrate des Newton-Raphson-Verfahrens ist mit einer von der Stabilität des Gleichgewichtszustandes abhängigen Aktivierung der Dämpfung zu vermindern.

Mit dem Bogenlängenverfahren [157, 120] kann der gesamte Last-Verformungs-Pfad berechnet werden. Bei diesem Verfahren wird dem jeweiligen Gleichungssystem eine Nebenbedingung auferlegt, um der Korrektoriteration unter Berücksichtigung einer Änderung des Lastparameters $\lambda$ eine spezielle Richtung vorzuschreiben. Die Nebenbedingung kann die Iteration auf einer Normalenebene, Tangentenebene oder Kugelfläche fordern. Im Rahmen dieser Arbeit wird die Iteration auf einer Tangentenebene realisiert. Hierzu ist in der gemischten Formulierung das um die Nebenbedingung

$$
f(\mathbf{z}, \lambda)=\Delta \mathbf{z}_{P_{i}}^{T}\left(\mathbf{z}-\mathbf{z}_{i}\right)+\left(\lambda-\lambda_{i}\right)=0, \text { mit } i=1,2, \ldots
$$

erweiterte Gleichungssystem

$$
\widehat{\mathbf{G}}(\mathbf{z}, \lambda)=\left\{\begin{array}{c}
\mathbf{A}_{S}(\mathbf{z}) \mathbf{z}=\lambda \mathbf{p} \\
f(\mathbf{z}, \lambda)=0
\end{array}\right\}
$$

iterativ zu lösen, wozu die in [167] erläuterte Ränderungsmethode verwendet wird. Bild 2.7 links verdeutlicht das Prinzip der Iteration auf der Tangentenebene. Ausge-
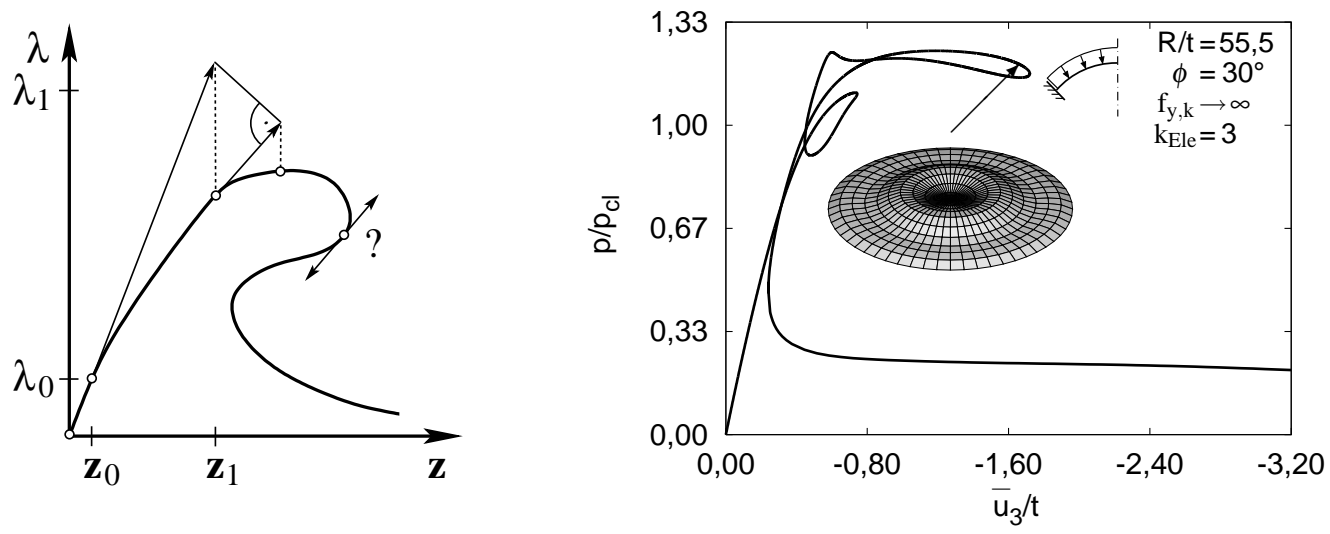

Bild 2.7: Bogenlängenverfahren - Prinzip und Anwendungsbeispiel

hend vom Gleichgewichtszustand 0 ist der Prädiktor $\Delta \mathbf{z}_{P_{0}}=\mathbf{z}_{1}-\mathbf{z}_{0}$ festzulegen. Anhand der Linearisierung des Gleichungssystems (2.58) und $\Delta \mathbf{z}_{P_{1}}=\mathbf{A}_{T}^{-1}\left(\mathbf{z}_{1}\right) \mathbf{p}$ ist eine erste Approximation des neuen Gleichgewichtszustandes ermittelbar. Die Iteration konvergiert in der Regel nach wenigen Schritten. Ein zu großer Prädiktor kann jedoch zur Divergenz führen und einen Neustart des Verfahrens mit verbessertem Prädiktor erfordern. Die Richtung der Pfadverfolgung wird mit der inkrementellen äußeren Arbeit festgelegt. Zur Verifikation der Implementierung des Verfahrens wird der LastVerformungs-Pfad einer Kugelschale unter Radialdruck berechnet, deren Materialparameter mit $E=2,06 \cdot 10^{5} \mathrm{~N} / \mathrm{mm}^{2}$ und $\nu=0,3$ gegeben sind. Die Diskretisierung des elastischen Potentials erfolgt anhand gemischter Elemente mit biquadratischen 
Ansätzen. Die Anzahl der Elemente ist unter Berücksichtigung des in Gl. (4.24) angegebenen Elementierungsgrades $k_{\text {Ele }}$ gewählt. Der Last-Verformungs-Pfad ist in Bild 2.7 rechts dargestellt und zeigt qualitativ gute Übereinstimmung mit der Referenzlösung [158], verdeutlicht aber auch die Komplexität der Randwertaufgabe.

\subsubsection{Eigenwertprobleme}

Die Bewertung finiter Elemente sowie der dynamischen Eigenschaften und der Stabilität von Systemen kann mit der Lösung von Eigenwertproblemen der Form

$$
[\mathbf{A}+\lambda \mathbf{B}] \boldsymbol{\phi}=\mathbf{0} \text { bzw. } \quad\left[\mathbf{A}+\lambda \mathbf{B}+\lambda^{2} \mathbf{C}\right] \boldsymbol{\phi}=\mathbf{0}, \text { mit } \mathbf{A}, \mathbf{B}, \mathbf{C} \in \mathbb{R}^{n \times n},
$$

erfolgen. Das hinsichtlich des Eigenwertes $\lambda$ lineare Eigenwertproblem impliziert für $\mathbf{B}=\mathbf{I}$ das spezielle Eigenwertproblem und ist mit verschiedenen Verfahren lösbar. Sind nur die betragsmäßig kleinsten Eigenwerte und die zugehörigen Eigenvektoren von Interesse, so ist zur deren Ermittlung insbesondere die inverse Vektoriteration geeignet. Dieses Verfahren verwendet für die Eigenvektoren die Normierung

$$
\boldsymbol{\phi}_{i}^{T} \mathbf{B} \boldsymbol{\phi}_{i}=1,0, \text { mit } i=1,2, \ldots, n_{\text {Eig }},
$$

und ist in mathematischer Hinsicht für positiv definite Matrizen abgesichert [130]. Jedoch können Konvergenzprobleme bei dicht benachbarten Eigenwerten auftreten. Für nicht positiv definite oder unsymmetrische Matrizen können neben reellen auch komplexe Eigenpaare existieren. In diesem Fall kann bei Systemen geringer Dimension ein Shiften des Eigenwertproblems die Identifikation eines reellen Eigenpaares ermöglichen. Der Betrag des erforderlichen Shiftes ist aber oft nicht direkt anzugeben.

Die Konvergenzrate der inversen Vektoriteration wird verringert, wenn die Matrizen des Eigenwertproblems vom Eigenvektor abhängig sind. Die Aktualisierung der Matrizen kann dabei in jedem Iterationsschritt oder nach Lösung des Eigenwertproblems, bei dem die Matrizen als konstant angesetzt werden, erfolgen. Die physikalische Bedeutung der Eigenwerte resultiert aus dem jeweiligen Eigenwertproblem. Komplexe Eigenwerte gehören zwar häufig zur Lösung des mathematischen Modells, repräsentieren aber keine physikalisch sinnvollen Lösungen der im Rahmen dieser Arbeit zu lösenden Eigenwertprobleme und werden daher nicht weiter berücksichtigt.

Für die Wahl eines Verfahrens zur Lösung eines hinsichtlich des Eigenwertes $\lambda$ quadratischen Eigenwertproblems ist wesentlich, ob dieses direkt gelöst oder mittels einer Identität in ein äquivalentes lineares Eigenwertproblem doppelter Dimension transformiert werden soll. Die Lösung des äquivalenten linearen Eigenwertproblems gelingt in der Regel mit der inversen Vektoriteration, wenn nur wenige der $2 n$ Eigenpaare komplex bzw. der Startvektor und der Shift adäquat gewählt sind. Ferner 
sind Unterraum-Verfahren wie das Arnoldi-Verfahren und Verfahren anwendbar, die alle Eigenpaare berechnen $[6,138]$. Letztere sind bei Systemen großer Dimension zwar ineffizient, gewährleisten aber die Identifikation des kleinsten bzw. größten Eigenwertes. Zur direkten Lösung quadratischer Eigenwertprobleme sind das JacobiDavidson-Verfahren und ein modifiziertes Arnoldi-Verfahren [150] geeignet.

Bei der Bewertung der Verfahren zur Lösung von Eigenwertproblemen ist in ausgewählten Fällen die Normierung der Eigenvektoren zu beachten. So z. B. bei dem in Kapitel 4 vorgestellten Störenergie-Konzept, wenn dieses im Rahmen einer gemischten Formulierung umgesetzt wird. In diesem Fall kann es bei Verwendung von Verfahren mit einer zur Normierung (2.60) unterschiedlichen Skalierung der Eigenvektoren notwendig werden, in jedem Iterationsschritt zuerst alle reellen Eigenpaare zu berechnen und dann das Eigenpaar zu ermitteln, das zum Minimum an Störenergie führt. Dieses Vorgehen ist erforderlich, wenn aufgrund der Normierung die Störenergie nicht allein mit dem Eigenwert beschreibbar ist und somit der kleinste positive Eigenwert nicht unbedingt mit dem Minimum an Störenergie einhergeht. Die Art der Normierung des Eigenvektors ist auch zu beachten, da bei der Umsetzung des Störenergie-Konzeptes im Rahmen einer Weggrößenformulierung die Matrix C quadratisch vom Eigenvektor abhängig ist. So sind bei Verwendung der Maximumnorm die Elemente des Eigenvektors u. U. sehr klein, womit die Elemente der Matrix gegen null tendieren und das Eigenwertproblem seine physikalische Bedeutung verliert. Mit einer geeigneten Normierung der Eigenpaare wäre dies zu vermeiden.

\subsubsection{Integration der Bewegungsgleichung}

Die Integration der semidiskreten Bewegungsgleichung (2.30) erfolgt mit dem als Sonderfall des verallgemeinerten $\alpha$-Verfahrens zu interpretierenden Newmark-Verfahren. Bei diesem impliziten direkten Einschrittverfahren werden die Verschiebungen und die Geschwindigkeiten zum Zeitpunkt $t+\Delta t$ approximiert mit

$$
\begin{aligned}
& \mathbf{u}_{t+\Delta t}=\mathbf{u}_{t}+\Delta t \dot{\mathbf{u}}_{t}+\left(\frac{1}{2}-\beta\right) \Delta t^{2} \ddot{\mathbf{u}}_{t}+\beta \Delta t^{2} \ddot{\mathbf{u}}_{t+\Delta t}, \\
& \dot{\mathbf{u}}_{t+\Delta t}=\dot{\mathbf{u}}_{t}+(1-\gamma) \Delta t \ddot{\mathbf{u}}_{t}+\gamma \Delta t \ddot{\mathbf{u}}_{t+\Delta t}
\end{aligned}
$$

Mit den Approximationen folgen aus der semidiskreten Bewegungsgleichung zum Zeitpunkt $t+\Delta t$ der effektive Lastvektor

$$
\begin{aligned}
\mathbf{p}_{\mathrm{eff}} & =\mathbf{p}_{t+\Delta t}+\mathbf{D}\left(\frac{\gamma}{\beta \Delta t} \mathbf{z}_{t}+\left(\frac{\gamma}{\beta}-1\right) \dot{\mathbf{z}}_{t}+\left(\frac{\gamma}{2 \beta}-1\right) \Delta t \ddot{\mathbf{z}}_{t}\right) \\
& +\mathbf{M}\left(\frac{1}{\beta \Delta t^{2}} \mathbf{z}_{t}+\frac{1}{\beta \Delta t} \dot{\mathbf{z}}_{t}+\left(\frac{1}{2 \beta}-1\right) \ddot{\mathbf{z}}_{t}\right)
\end{aligned}
$$


die effektive Systemmatrix

$$
\mathbf{A}_{S, \text { eff }}=\mathbf{A}_{S}+\frac{\gamma}{\beta \Delta t} \mathbf{D}+\frac{1}{\beta \Delta t^{2}} \mathbf{M}
$$

und die effektive Strukturgleichung

$$
\mathbf{A}_{S, \text { eff }} \mathbf{z}=\mathbf{p}_{\text {eff }} .
$$

Bei Verwendung der Rayleigh-Dämpfung ist zur Berechnung steifigkeitsproportionaler Elemente der Dämpfungsmatrix $\mathbf{D}$ die Matrix $\mathbf{A}_{T}$ in eine Steifigkeitsmatrix zu transformieren. Die Beschleunigung $\ddot{\mathbf{u}}_{0}$ wird durch Auswertung der Bewegungsgleichung zum Zeitpunkt $t_{0}$ bestimmt. Die Lösung der effektiven Strukturgleichung wird mit dem Newton-Raphson-Verfahren ermittelt und ermöglicht mit den Gln. (2.61) die Berechnung der Geschwindigkeit und Beschleunigung zum Zeitpunkt $t+\Delta t$. Die Parameter $\beta, \gamma$ beeinflussen die Stabilität und Genauigkeitsordnung des Newmark-Verfahrens. Für die gewählten Parameter $\beta=0,25, \gamma=0,5$ wird die Beschleunigung im Zeitschritt mit einem konstanten Verlauf approximiert. Bei linearem Tragverhalten ist hiermit unbedingte Stabilität sowie eine Genauigkeit zweiter Ordnung erzielbar. Ferner wird numerische Dissipation, aber nicht numerische Dispersion vermieden [12].

Bei geometrisch nichtlinearem Tragverhalten ist das Newmark-Verfahren energetisch nicht stabil. Somit kann algorithmisch bedingt die Energie eines Systems auch bei autonomen Bewegungen zunehmen und das Tragverhalten nicht hinreichend genau beschrieben werden. Folglich ist eine Unterscheidung von numerischer und physikalischer Instabilität u.U. nicht möglich und das Newmark-Verfahren zur Bewertung des Langzeitverhaltens von Systemen nur bedingt geeignet. Numerische Stabilität ist sowohl mit kontrollierter numerischer Dissipation als auch mit erzwungener Energie-, Impuls- und Drehimpulserhaltung im Zeitschritt zu erreichen [137, 99], wobei der Diskretisierungsfehler allerdings in anderer Form auftritt. Der im Newmark-Verfahren für die gewählten Parameter $\beta, \gamma$ auftretende Fehler wird mit abnehmender, eventuell sogar adaptiv gesteuerter Zeitschrittweite kleiner [12].

\subsubsection{Funktionalanalytische Betrachtungen}

Bei linearem Systemverhalten setzt die Lösbarkeit einer Variationsaufgabe die Existenz und die Eindeutigkeit der Lösung voraus und ist das in Gl. (2.10) angegebene Prinzip vom Stationärwert des Gesamtpotentials dem Minimierungsproblem

$$
\inf _{u \in V_{u}} J(u), \text { mit } J(u)=\frac{1}{2} a(u, u)-g(u),
$$

äquivalent. Dabei repräsentiert die Variable $u$ Verschiebungsfelder bzw. Elemente eines geeigneten Hilbert-Raumes $V_{u}$. Im Gegensatz zur Linearform $g(u)$ sind die Ei- 
genschaften der Bilinearform $a$ entscheidend für die Lösbarkeit des Minimierungsproblems. Dem Satz von Lax-Milgram [19] zufolge existiert die eindeutige Lösung, wenn die Bilinearform stetig und symmetrisch ist sowie die Elliptizitätsbedingung

$$
a(u, u) \geq \alpha\|u\|^{2} \quad \forall u \in V_{u}, \alpha>0,
$$

erfüllt. Die Elliptizitätsbedingung gewährleistet die Konvexität des Funktionals $J(u)$ und stellt sicher, dass für jedes Verschiebungsfeld eines gelagerten Systems die Formänderungsenergie nach unten beschränkt ist. Aus der Diskretisierung der Bilinearform resultiert die Systemmatrix, deren positive Definitheit nur eine Bedingung für die Stabilität der Lösung ist. Eine weitere Bedingung ist die Existenz eines oberen und unteren Grenzwertes für die Eigenwerte der Systemmatrix [68]. Fehlende Elliptizität, z. B. infolge reduzierter Integration, ist Ursache von Nullenergiemoden. Für unsymmetrische Bilinearformen liegt indes kein Minimierungsproblem vor [21].

Das Prinzip von Hellinger/Reissner (2.11) beschreibt ein Sattelpunktproblem und ist für nicht gebettete lineare Systeme gleichwertig zu

$$
\inf _{S \in V_{S}} \sup _{u \in V_{u}} \widetilde{L}(S, u) \text {, mit } \widetilde{L}(S, u)=-\frac{1}{2} a(S, S)+b(S, u)-f(S)-g(u),
$$

wobei $V_{S}$ den Raum der zulässigen Spannungsfelder $S$ darstellt. Die Lösbarkeit des Sattelpunktproblems und die Stabilität der Lösung sind primär von den Eigenschaften der Bilinearformen $a$ und $b$ abhängig. Hierfür wesentliche Bedingungen werden nachfolgend anhand der ersten Variation des diskretisierten Funktionals $\widetilde{L}$ aufgezeigt. Dazu ist es zweckmäßig, in Anlehnung an Gl. (2.31) die Matrizen $\mathbf{F}_{D}, \mathbf{F}_{B}$ und $\mathbf{0}$ zur Matrix $\check{\mathbf{A}}$ und die Matrizen $\mathbf{D}$ und $\mathbf{B}$ zur Matrix $\check{\mathbf{B}}$ zusammenzufassen. Ferner werden die Freiwerte der räumlichen Diskretisierung der Spannungsfelder mit dem Vektor $\mathbf{s}$ und die aus eingeprägten Verzerrungen resultierenden arbeitsäquivalenten Knoteneinwirkungen mit dem Vektor $\gamma$ erfasst. Die Zerlegung des Raumes $V_{\mathbf{s}}$ in die Orthogonalräume $V_{\mathbf{s} 0}$ und $V_{\mathbf{s} \perp}$ gemäß

$$
V_{\mathbf{s}}=V_{\mathbf{s} 0} \oplus V_{\mathbf{s} \perp}, \text { mit } V_{\mathbf{s} \perp}:=\left(V_{\mathbf{s} 0}\right)^{\perp}, V_{\mathbf{s} 0}:=\left\{\mathbf{s} \in V_{\mathbf{s}} \mid \check{\mathbf{B}}^{T} \mathbf{s}=\mathbf{0}\right\},
$$

ermöglicht eine entsprechende Zerlegung der Matrizen $\check{\mathbf{A}}$ und $\check{\mathbf{B}}$ sowie der Vektoren $\mathbf{s}$ und $\gamma$. Da der Unterraum $V_{\mathbf{s} 0}$ dem Kern der Matrix $\check{\mathbf{B}}^{T}$ entspricht, beeinflusst die Matrix $\check{\mathbf{B}}_{0}^{T}$ die Lösung des Sattelpunktproblems nicht. Somit ist das aus der ersten Variation des diskretisierten Funktionals $\widetilde{L}$ folgende Gleichungssystem unter Beachtung der Dimension der Räume in die Form gemäß Gl. (2.69) rechts transformierbar.

$$
\left[\begin{array}{ccc}
-\mathbf{F}_{D} & \mathbf{0} & \mathbf{D} \\
\mathbf{0} & -\mathbf{F}_{B} & \mathbf{B} \\
\mathbf{D}^{T} & \mathbf{B}^{T} & \mathbf{0}
\end{array}\right]\left[\begin{array}{c}
\mathbf{n} \\
\mathbf{m} \\
\mathbf{u}
\end{array}\right]=\left[\begin{array}{c}
\widehat{\alpha} \\
\widehat{\beta} \\
\widehat{\mathbf{p}}
\end{array}\right] \Longleftrightarrow\left[\begin{array}{ccc}
\check{\mathbf{A}}_{00} & \check{\mathbf{A}}_{0 \perp} & \mathbf{0} \\
\check{\mathbf{A}}_{\perp 0} & \check{\mathbf{A}}_{\perp \perp} & \check{\mathbf{B}}_{\perp} \\
\mathbf{0} & \check{\mathbf{B}}_{\perp}^{T} & \mathbf{0}
\end{array}\right]\left[\begin{array}{c}
\mathbf{s}_{0} \\
\mathbf{s}_{\perp} \\
\mathbf{u}
\end{array}\right]=\left[\begin{array}{c}
\gamma_{0} \\
\gamma_{\perp} \\
\widehat{\mathbf{p}}
\end{array}\right]
$$


Notwendig und hinreichend für die eindeutige Lösung des Sattelpunktproblems sind die Existenz der Inversen der Matrix $\check{\mathbf{A}}_{00}$ und der Matrix $\check{\mathbf{B}}_{\perp}$ [63]. Die Matrix $\check{\mathbf{A}}$ muss folglich nur in dem Bereich den notwendigen Rang der Koeffizientenmatrix gewährleisten, der von der Matrix $\check{\mathbf{B}}_{\perp}$ nicht erfasst wird. Die Inverse der Koeffizientenmatrix muss indes nicht existieren. Damit die ermittelte Lösung auch stabil ist und somit die Zustandsgrößen bei einer kleinen Änderung der Belastung auch nur eine kleine Änderung erfahren, muss die Norm beider inversen Matrizen nach oben beschränkt sein. Diese Bedingung ist äquivalent den inf-sup-Bedingungen

$$
\inf _{\mathbf{s}_{1} \in V_{\mathbf{s} 0}} \sup _{\mathbf{s}_{2} \in V_{\mathbf{s} 0}} \frac{\left|\mathbf{s}_{1}^{T} \check{\mathbf{A}} \mathbf{s}_{2}\right|}{\left\|\mathbf{s}_{1}\right\|\left\|\mathbf{s}_{2}\right\|} \geq \gamma_{\alpha}>0, \quad \inf _{\mathbf{u} \in V_{\mathbf{u}}} \sup _{\mathbf{s} \in V_{\mathbf{s}}} \frac{\left|\mathbf{u}^{T} \check{\mathbf{B}}^{T} \mathbf{s}\right|}{\|\mathbf{u}\|\|\mathbf{s}\|} \geq \gamma_{\beta}>0 .
$$

Mit den Bedingungen wird die Elliptizität des Funktionals $\widetilde{L}$ bezüglich der Variablen $\mathbf{s}$ und $\mathbf{u}$ gefordert. Darüber hinaus sind zwei Bedingungen zu erfüllen, um die Kontinuität des Funktionals sicherzustellen [16]. Die Konstanten $\gamma_{\alpha}$ und $\gamma_{\beta}$ sind, sofern die Lösung stabil ist, unabhängig von der Diskretisierung. Im Gegensatz zur ersten Bedingung (2.70) berücksichtigt die zweite Bedingung die Spannungs- und die Verschiebungsansätze. Daher kann für diese Bedingung eine anschauliche Interpretation angegeben werden. Demnach ist die Diskretisierung derart zu wählen, dass zu jedem Verzerrungsmode mindestens ein nicht orthogonaler Spannungsmode existiert [5]. Ansonsten sind Instabilität und Nullenergiemoden nicht auszuschließen.

Die inf-sup-Bedingungen und die Kontinuitätsbedingungen sind zwar hinreichend, um die Stabilität der Lösung des Gleichungssystems (2.69) bzw. der FE-Formulierung nachzuweisen. Da der Nachweis über die Einhaltung der inf-sup-Bedingungen nicht leicht zu erbringen ist, begnügt man sich oft mit der notwendigen Bedingung, dass ein diskretisiertes Sattelpunktproblem mindestens so viele Spannungsfreiwerte wie Verschiebungsfreiwerte abzüglich der Anzahl der Starrkörpermoden aufweisen soll [67, 68]. Die Bedingung ist für die im Rahmen dieser Arbeit verwendeten gemischten Elemente erfüllt. Die inf-sup-Bedingungen beziehen sich auf das Gleichungssystem (2.69) und somit auf nicht gebettete lineare Systeme. Für andere Systeme und somit andere Belegungen der Koeffizientenmatrix wird der Nachweis über die Einhaltung der Bedingungen, sofern diese dann noch relevant sind, nicht leichter. Daher ist es dem Autor nicht möglich, die Stabilität der verwendeten Elemente allgemeingültig nachzuweisen. Vielmehr wird die Stabilität der Lösung eines Sattelpunktproblems anhand deren Entwicklung bei verschiedenen Diskretisierungen bewertet.

Die Sattelpunkteigenschaft des Funktionals $\widetilde{L}$ bedingt in der gemischten Formulierung negative Eigenwerte der Systemmatrix. Somit ist die Matrix nicht positiv definit. Die Anzahl negativer Eigenwerte entspricht in der Regel der Anzahl der Spannungsfreiwerte. Der einem negativen Eigenwert zugehörige Eigenvektor kann eine Gleichgewichtsgruppe repräsentieren [65]. Eine Gleichgewichtsgruppe ist dadurch charak- 
terisiert, dass zumindest einige Spannungsfreiwerte ungleich null und sämtliche Verschiebungsfreiwerte identisch null sind, das Gleichgewicht des Systems aber in integraler Form erfüllt wird. Eine Gleichgewichtsgruppe ist damit das Pendant zu einem Starrkörpermode. Die einem Sattelpunkt zugehörige Formänderungsenergie ist mit Hilfe klassischer Variationsprinzipien einzuschranken [66]. Dahingegen können allgemeingültige Hinweise zur Topologie der Energiefläche eines Systems im Bereich indifferenter und instabiler Gleichgewichtszustände nicht angegeben werden.

\subsubsection{Versteifungseffekte}

FE-Lösungen können infolge numerisch bedingter Versteifungen merkliche Fehler aufweisen. Diese Versteifungseffekte sind unter dem Begriff Locking zusammengefasst. Eine Klassifizierung von Locking ist in mechanischer, mathematischer und numerischer Hinsicht möglich [13]. In mechanischer Hinsicht charakterisieren parasitäre Spannungen bzw. Verzerrungen das Locking. In mathematischer Hinsicht dominiert ein kritischer Parameter das der Elementformulierung zugrunde liegende Funktional. Eine Bewertung von Elementen hinsichtlich der Tendenz zum Locking gelingt mit einem Zwangsbedingungsfaktor [77]. Ursache für Locking ist die unausgewogene Approximation bestimmter Feldgrößen. Das Auftreten von Locking ist somit von der Elementformulierung abhängig. Mit zunehmender Ordnung der Ansätze und feinerer Diskretisierung verringern sich viele Versteifungseffekte.

$\mathrm{Zu}$ den bei der 5- bzw. 3-Parameter-Schalentheorie auftretenden Versteifungseffekten zählen das Schub-, Querschub- und Membran-Locking. Für Schub-Locking sind parasitäre Schubverzerrungen bei bestimmten ebenen Deformationsmoden von Elementen mit bilinearen Ansätzen kennzeichnend. Kritischer Parameter ist das Seitenverhältnis der Elemente. Querschub-Locking tritt bei Elementen auf Basis der Reissner-Mindlin-Theorie auf und ist durch parasitäre Querschubverzerrungen charakterisiert. Membran-Locking bewirkt bei reiner Biegebeanspruchung gekrümmter Schalentragwerke parasitäre Membranverzerrungen in der FE-Lösung. Membranund Querschub-Locking sind von der Schlankheit der Elemente abhängig.

Methoden zur Verringerung von Locking zielen darauf ab, den Verlauf der parasitären Verzerrungen zu beeinflussen. Dabei sind zwei Vorgehensweisen zu unterscheiden: Die Elimination parasitärer Anteile aus der Elementformulierung und die Ergänzung der Elementformulierung um zusätzliche Verzerrungsanteile, mit denen eine ausgewogene Balance zwischen den entsprechenden Feldgrößen herstellbar ist. Geeignete Methoden sind die reduzierte Integration, die Assumed-Natural-Strain-Methode (ANS), die Enhanced-Assumed-Strain-Methode (EAS) und die Discrete-Strain-GapMethode (DSG). Die Methoden nutzen die Tatsache, dass der Verlauf parasitärer Verzerrungen Nullstellen aufweist und für bestimmte Richtungen im Integral oftmals 
identisch null ist. Die Effizienz der Methoden ist von der Belastung und der Netzverzerrung abhängig. Somit ist nicht auszuschließen, dass bei bestimmten Belastungen und sehr unregelmäßigen Elementgeometrien parasitäre Verzerrungen entstehen.

Die reduzierte Integration ist auf alle oder einzelne Terme der Elementmatrix anzuwenden [168]. Der einfachen numerischen Umsetzung steht das mögliche Auftreten von Nullenergiemoden als wesentlicher Nachteil gegenüber. Diesem kann bei einer Weggrößenformulierung mit einer Hourglass-Stabilisierung begegnet werden [49]. Im Rahmen dieser Arbeit wird primär die selektiv reduzierte Integration angewendet.

Die ANS-Methode beinhaltet die Formulierung modifizierter Verzerrungen, die aus der Interpolation der Verzerrungen an ausgewählten Kollokationspunkten folgen [78]. An diesen Punkten sind die parasitären Verzerrungen identisch null. Anzahl und Position der Punkte sind von der Elementformulierung und dem Locking-Effekt abhängig. Die Methode wird oft zur Verringerung des Querschub-Lockings verwendet.

Grundgedanke der EAS-Methode ist die Berücksichtigung zusätzlicher Verzerrungsfelder [136]. Diese Verzerrungsfelder müssen auf Elementebene orthogonal zu den Spannungen sein. Somit können die Spannungen aus dem reparametrisierten Prinzip von $\mathrm{Hu}-W a s h i z u$ eliminiert werden. Ein Nachteil der Methode ist die oftmals nicht einfache Identifikation der zusätzlichen Verzerrungsfelder. Ein weiterer Nachteil ist die zur statischen Kondensation der Freiwerte der zusätzlichen Verzerrungsfelder auf Elementebene erforderliche Invertierung der Matrix, welche die Produkte der Ansatzfunktionen für den Verlauf der Verzerrungsfelder enthält. Die EAS-Methode kann zur Verringerung sämtlicher Versteifungseffekte eingesetzt werden.

Die DSG-Methode diente ursprünglich der Modifikation der Querschubverzerrungen, erlaubt aber die Verringerung aller geometrischen Versteifungseffekte [15, 91]. Die Methode impliziert drei Schritte. Mittels Integration der jeweiligen kinematischen Gleichung entlang den Koordinatenlinien $\theta^{\alpha}$ sind die Gaps an den Knoten $k$ zu bestimmen. Die Gaps repräsentieren Relativverformungen zwischen den Knoten und weisen keine Verformungsanteile infolge parasitärer Verzerrungen auf. Interpolation der Gaps mit den Ansatzfunktionen $N^{k}$ führt auf das Gap-Feld und dessen Differentiation nach den konvektiven Koordinaten auf die modifizierten Verzerrungen,

$$
\alpha_{\alpha \alpha}^{D S G}=N_{, \alpha}^{K} \int_{\theta_{0}^{\alpha}}^{\theta_{K}^{\alpha}} \alpha_{\alpha \alpha} d \theta^{\alpha} \text { bzw. } \alpha_{12}^{D S G}=N_{, 2}^{L} \int_{\theta_{0}^{2}}^{\theta_{L}^{2}}\left(N_{, 1}^{K} \int_{\theta_{0}^{1}}^{\theta_{K}^{1}} \alpha_{12} d \theta^{1}\right) d \theta^{2}
$$

Modifizierte Querschubverzerrungen sind entsprechend den modifizierten Normalverzerrungen zu berechnen. Der wesentliche Vorteil der DSG-Methode gegenüber den Assumed-Strain-Methoden ist das einheitliche, von der Elementformulierung unabhängige Vorgehen zur Verringerung von Versteifungseffekten. Die Verifikation der 
DSG-Methode für den Fall des Membran-Lockings erfolgt anhand des in Bild 2.8 dargestellten Systems, wobei lineares Tragverhalten vorausgesetzt wird. Zur Diskretisierung des elastischen Potentials werden fünf gemischte Elemente mit biquadratischen Ansätzen und gleicher Geometrie verwendet.
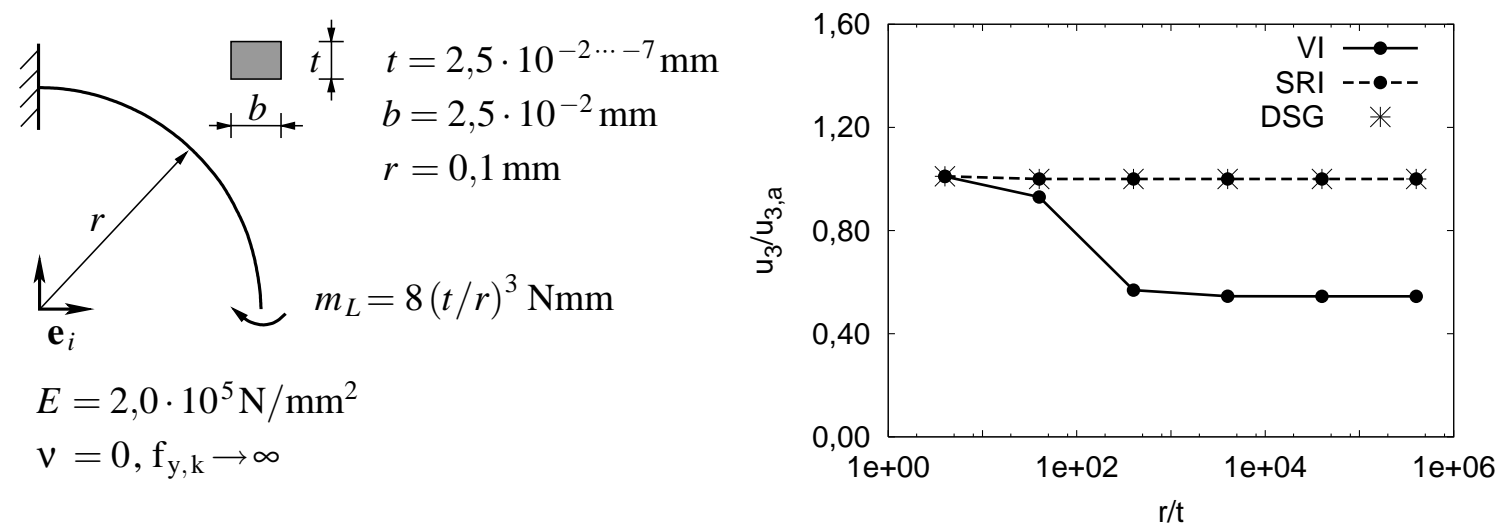

Bild 2.8: System und Verformungsverhalten vs. Schlankheit

Ohne Elimination der parasitären Membranverzerrungen und somit bei voller Integration (VI) sämtlicher Terme der ursprünglichen Elementmatrizen ist die ermittelte radiale Verschiebung $u_{3}$ des Lastangriffspunktes signifikant von der Schlankheit $r / t$ abhängig. Dabei tendiert die radiale Verschiebung mit zunehmender Schlankheit gegen einen im Vergleich zur analytischen Lösung $u_{3, a}$ deutlich kleineren Wert. Bei Anwendung der DSG-Methode als auch der selektiv reduzierten Integration(SRI) wird die analytische Lösung für die angegebenen Schlankheiten gut angenähert. 


\section{Stabilität von Systemen}

Physikalische, aber auch biologische und wirtschaftliche Systeme sind oft hinsichtlich ihrer Stabilität zu bewerten. Stabilität ist eng mit der Wirkung von Störungen verbunden. Die Analyse von Systemen und Systemantworten ermöglicht die Definition geeigneter Stabilitätskriterien. Nachfolgende Ausführungen berücksichtigen die im Rahmen dieser Arbeit herausragende Stellung von Schalentragwerken.

\subsection{Bewegungen und Attraktoren}

Bewegungen zeigen das Verhalten von Systemen auf und sind hinsichtlich Anregungsmechanismus und Langzeitverhalten zu unterscheiden. Bewegungen sind in vielen Fällen Schwingungen. Freie Schwingungen charakterisieren das Verhalten eines Systems infolge der Anfangsbedingungen. Selbsterregte Schwingungen können aus der Wechselwirkung eines Systems mit einem anderen System, z.B. dem umströmenden Medium, folgen. Die Intensität der Wechselwirkung und der damit einhergehende Energietransfer werden entscheidend von der Relativgeschwindigkeit zwischen den Systemen beeinflusst. Freie und selbsterregte Schwingungen kennzeichnen autonome Systeme. Erzwungene Schwingungen werden durch zeitabhängige Belastungen initiiert. Entspricht bei periodischer Belastung die Erregerfrequenz einer Eigenkreisfrequenz des Systems, so tritt Resonanz ein. Parametererregte Schwingungen resultieren bei periodischer Belastung aus der schnellen Änderung von Koeffizienten des Differentialgleichungssystems und können parametrische Resonanz für unterschiedliche Konstellationen der Systemparameter bewirken. Diese Schwingungen entsprechen hinsichtlich Form und Frequenz nicht der zur Belastung affinen Schwingung. Erzwungene und parametererregte Schwingungen charakterisieren infolge der Fremderregung heteronome Systeme. Für das Verhalten von Systemen ist in vielen Fällen das gleichzeitige Auftreten verschiedener Arten von Schwingungen kennzeichnend.

Das Langzeitverhalten nichtlinearer konservativer Systeme ist aufgrund ständigen Energieaustausches zwischen verschiedenen Schwingungsmoden oft nicht hinreichend bewertbar. Bei dissipativen Systemen wird das Langzeitverhalten in der Regel von Attraktoren dominiert. Ein Attraktor ist die Grenzmenge aller Bewegungen innerhalb seines Einzugsgebietes und durch Invarianz und Rekurrenz der Trajektorie 
gekennzeichnet [94]. Attraktoren können nebeneinander existieren und sind u. U. erst mit fortschreitender Zeitverlaufsberechnung identifizierbar. Zur Klassifizierung von Attraktoren sind z.B. Phasenporträts, Poincaré-Schnitte und Ljapunow-Exponenten geeignet. Zu den wesentlichen Typen von Attraktoren zählen neben Gleichgewichtszuständen auch Grenzzykel, Torus- und seltsame Attraktoren.

\subsection{Stabilitätsdefinitionen}

Die Stabilität von Bewegungen ist anhand der Wirkung von Störungen bewertbar. In Abhängigkeit vom Typ einer Bewegung sowie von der Art und des Zeitpunktes einer Störung existieren unterschiedliche Stabilitätsdefinitionen.

Die Stabilitätsdefinition nach Ljapunow gilt für diskrete Systeme, deren Verhalten mit dem Differentialgleichungssystem $\dot{\mathbf{x}}=\mathbf{f}(\mathbf{x}, t)$ beschrieben ist. Für kontinuierliche Systeme wird diese bei Wahl einer geeigneten Norm abgeschwächt [102]. Demnach heißt die ungestörte Bewegung $\mathbf{x}(t)$ (schwach) stabil, wenn für jedes beliebig kleine $\varepsilon>0$ ein $\delta(\varepsilon)>0$ existiert, so dass jede gestörte Bewegung $\mathbf{x}_{p}(t)$ mit

$$
\left\|\mathbf{x}\left(t_{0}\right)-\mathbf{x}_{p}\left(t_{0}\right)\right\|<\delta(\varepsilon)
$$

die Bedingung

$$
\left\|\mathbf{x}(t)-\mathbf{x}_{p}(t)\right\|<\varepsilon
$$

für alle Zeiten $t \geq t_{0}$ erfüllt. Gilt zusätzlich

$$
\lim _{t \rightarrow \infty}\left\|\mathbf{x}(t)-\mathbf{x}_{p}(t)\right\|=0,
$$

so heißt die ungestörte Bewegung asymptotisch stabil. Ist Bedingung (3.2) nicht erfüllt, so ist die ungestörte Bewegung instabil. Eine geometrische Interpretation der Stabilitätsdefinition nach Ljapunow ist in Bild 3.1 gegeben.

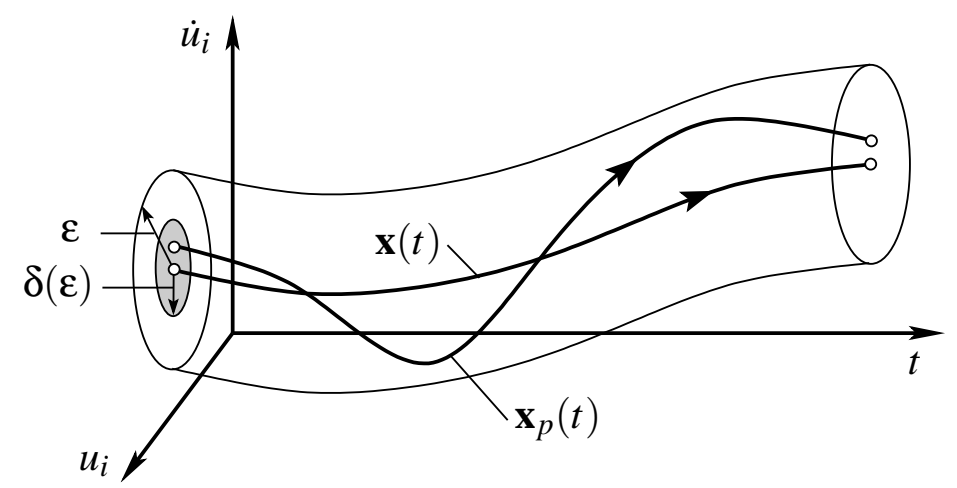

Bild 3.1: Stabilitätsdefinition nach Ljapunow

Allgemeingültige Hinweise zur Größe der $\varepsilon$-Umgebung sind nur schwer anzugeben. Die Bewertung der Stabilität einer Bewegung kann vielmehr in Abhängigkeit von der 
Wahl der $\varepsilon$-Umgebung erfolgen. Die praktische Bedeutung der Stabilitätsdefinition nach Ljapunow ist daher auf die Bewertung der Stabilität im Kleinen, d.h. relativ zur untersuchten Systemantwort, zu reduzieren [111]. Darüber hinaus wird eine Bewegung bereits bei temporärer Verletzung der Bedingung (3.2) als instabil identifiziert. Die Definition der Stabilität von Gleichgewichtszuständen ist als Sonderfall in der Stabilitätsdefinition nach Ljapunow enthalten.

Die Stabilitätsdefinition nach Ljapunow ist zur Bewertung der Stabilität von Bewegungen vielfach zu streng. Für periodische Bewegungen ist deshalb die Definition orbitaler Stabilität sinnvoll. Diese wird auch als Stabilität nach Poincaré bezeichnet. Die ungestörte Bewegung $\mathbf{x}(t)$ heißt orbital stabil, wenn für jedes beliebig kleine $\varepsilon>0$ ein $\delta(\varepsilon)>0$ und eine Zeitfunktion $t_{1}(t) \geq t_{0}$ existieren, so dass jede gestörte Bewegung $\mathbf{x}_{p}(t)$ mit

$$
\left\|\mathbf{x}\left(t_{0}\right)-\mathbf{x}_{p}\left(t_{0}\right)\right\|<\delta(\varepsilon)
$$

die Bedingung

$$
\left\|\mathbf{x}\left(t_{1}\right)-\mathbf{x}_{p}(t)\right\|<\varepsilon
$$

für alle Zeiten $t \geq t_{0}$ erfüllt. Entsprechend Bedingung (3.3) ist asymptotische orbitale Stabilität zu definieren. Im Unterschied zur Stabilitätsdefinition nach Ljapunow darf die Berechnung der Norm der Differenz zwischen ungestörter und gestörter Bewegung mit Bewegungszuständen unterschiedlicher Zeitpunkte erfolgen. Somit ist eine Bewegung, die stabil im Sinne von Ljapunow ist, auch orbital stabil.

Stabilität nach Ljapunow und orbitale Stabilität erfassen den Einfluss anfänglicher Störungen auf das Verhalten einer Bewegung. In Realität treten auch während einer Bewegung Störungen auf. Bei ständig wirkenden Störungen $\mathbf{R}_{s}(\mathbf{x}, t)$ ist Bedingung (3.3) hinreichend für die Stabilität von Gleichgewichtszuständen und periodischen Bewegungen. Für beliebige Bewegungen, die dem Differentialgleichungssystem $\dot{\mathbf{x}}=\mathbf{f}(\mathbf{x}, t)+\mathbf{R}_{s}(\mathbf{x}, t)$ genügen, gibt Duboschin [104] eine Stabilitätsdefinition an, die eine Verallgemeinerung der Stabilitätsdefinition nach Ljapunow darstellt.

Mit den vorgestellten Stabilitätsdefinitionen sind unterschiedliche Arten des Verlustes der Stabilität einer Bewegung erfassbar. Dieser tritt bei konservativen Systemen aufgrund Divergenz der Bewegung ein. Bei Systemen mit nichtkonservativer Belastung ist Instabilität auch infolge angefachter Schwingungen möglich [10]. Die Stabilitätsdefinitionen erlauben Aussagen zur Stabilität einer Bewegung für ein offenes Zeitintervall. Dies erfordert u. U. erheblichen Berechnungsaufwand. Eine Alternative ist die Bewertung der Stabilität für ein praktisch relevantes endliches Zeitintervall [101].

Neben den vorgestellten Stabilitätsdefinitionen sind weitere von Bedeutung. Strukturstabilität beschreibt den Einfluss der Änderung von Systemparametern wie Belas- 
tung und Dämpfung auf das Verhalten eines Systems. Bei Systemen mit stochastischer Verteilung der Systemparameter oder Störungen erfolgt die Bewertung der Stabilität mit Hilfe stochastischer Methoden. Dieses Vorgehen führt auf den Begriff der stochastischen Stabilität. Für Gleichgewichtszustände konservativer Systeme ist die Definition der Stabilität mit Hilfe energetischer Betrachtungen möglich [36]. Metastabilität sowie Stabilität im Kleinen bzw. praktische Instabilität beschreiben die Relativität von Aussagen zur Stabilität von Bewegungen nichtlinearer Systeme. Allgemein sind Stabilitätsaussagen relativ zum jeweiligen Bezugssystem.

\subsection{Lösungsmethoden}

Zur Lösung von Stabilitätsproblemen sind verschiedene Konzepte geeignet; zwei wichtige entwickelt Ljapunow. Die erste - auch indirekte - Methode erfordert auch für das gestörte System die Integration des zugrunde liegenden Differentialgleichungssystems. Die Methode basiert auf der unmittelbaren Betrachtung der gestörten Bewegung und erlaubt eine Bewertung der Differenz zwischen ungestörter und gestörter Bewegung. Die zweite - auch direkte - Methode zielt auf die Bewertung der Stabilität ohne Kenntnis der gestörten Bewegung. Der Methode liegen Ljapunow-Funktionale zugrunde. Existiert ein derartiges systemspezifisches Funktional, ist es positiv definit und dessen Zeitableitung negativ semidefinit, so ist die ungestörte Bewegung stabil. Zur Herleitung von Ljapunow-Funktionalen gibt es keine allgemeingültigen Regeln. Für konservative Systeme ist mit dem Hamilton-Funktional ein entsprechendes Funktional gegeben. Die Zeitableitung dieses Funktionals ist identisch null, was Energieerhaltung im System gewährleistet. Für statische Systeme ist das Funktional äquivalent der negativen Lagrange-Funktion bzw. dem elastischen Potential. Die Bewertung der Stabilität von Gleichgewichtszuständen konservativer Systeme wird somit auf die Untersuchung der Definitheit des elastischen Potentials reduziert. Dies entspricht dem Lagrange-Dirichlet-Theorem [10]. Für nichtkonservative Systeme ist die Verwendung eines verallgemeinertes Hamilton-Funktionals möglich [102].

\subsection{Stabilitätskriterien}

In der Literatur sind verschiedene Stabilitätskriterien angegeben. Die Kriterien basieren oftmals auf den beiden Methoden nach Ljapunow und sind u. a. hinsichtlich Anwendbarkeit und Zeitpunkt der Identifikation des Stabilitätsverlustes zu unterscheiden. Einige Stabilitätskriterien werden nachfolgend vorgestellt.

Die Bewertung der Stabilität von Bewegungen kann mit Hilfe von Ljapunow-Exponenten erfolgen. Ein Ljapunow-Exponent ist ein Maß für die mittlere exponentielle 
Konvergenz oder Divergenz zwischen ungestörter und gestörter Bewegung und zu

$$
\vartheta=\lim _{t \rightarrow \infty} \frac{1}{t} \ln \frac{\left\|\Delta \mathbf{x}_{p}(t)\right\|}{\left\|\mathbf{x}\left(t_{0}\right)-\mathbf{x}_{p}\left(t_{0}\right)\right\|}
$$

definiert. Dabei wird die zeitliche Entwicklung einer Störung zum Zeitpunkt $t_{0}$ mit $\Delta \mathbf{x}_{p}(t)=\mathbf{x}(t)-\mathbf{x}_{p}(t)$ ausgedrückt und oft mit dem linearen Differentialgleichungssystem $\Delta \dot{\mathbf{x}}_{p}(t)=\partial \mathbf{f} / \partial \mathbf{x} \Delta \mathbf{x}_{p}(t)$ beschrieben. Für ein System der Dimension $N=2 n$ sind $N$ linear unabhängige Störvektoren denkbar. Aus dem Spektrum der resultierenden Ljapunow-Exponenten ist der größte Ljapunow-Exponent für die Stabilität einer Bewegung entscheidend. Ein Ljapunow-Exponent größer null charakterisiert eine instabile Bewegung. Zur Berechnung eines Ljapunow-Exponenten ist eine Zeitverlaufsberechnung erforderlich, deren Dauer von dessen Konvergenz abhängig ist. Die Summe aller $N$ Ljapunow-Exponenten beschreibt die mittlere Divergenz des Flusses eines Systems und ist für konservative Systeme identisch null. Ein dem LjapunowExponenten ähnliches Stabilitätsmaß stellt der Poincaré-Multiplikator dar [112].

Die Stabilität periodischer Bewegungen kann auch mit Hilfe der Floquet-Theorie bewertet werden. Dabei wird die zeitliche Entwicklung der $N$ linear unabhängigen Störvektoren innerhalb einer Periode mit einer Übertragungsmatrix, der Monodromiematrix, beschrieben. Die Eigenwerte dieser Matrix werden als charakteristische Multiplikatoren bezeichnet. Ist der Spektralradius der Monodromiematrix kleiner eins, so ist die ungestörte Bewegung asymptotisch stabil und repräsentiert einen stabilen Grenzzykel. Eine Verringerung der Dimension der Monodromiematrix und somit des u.U. beträchtlichen Aufwandes zu deren Berechnung ist durch Approximation der Störvektoren mit den dominanten Eigenschwingungsmoden möglich [42].

Kriterien zur Identifikation der kinetischen Grenzlast beschreiben oftmals die Stabilität eines Gleichgewichtszustandes im Fall einer kinetischen Störung und können auf der zeitlichen Entwicklung charakteristischer Verformungen basieren. Budiansky und Roth [24] schlagen ein entsprechendes Kriterium zur Identifikation der kinetischen Grenzlast flacher Kugelschalen unter Radialdruck für den Fall rotationssymmetrischen Beulens vor. Das Kriterium basiert auf der signifikanten Änderung des Verformungsverhaltens beim Durchschlagen und erfordert Zeitverlaufsberechnungen von vorab unbestimmter Dauer. Die Wahl ungeeigneter charakteristischer Verformungen kann aber zur falschen Bewertung der Stabilität führen. Burmeister [25] entwickelt die Skalierungsmethode, bei der begleitend zur Zeitverlaufsberechnung ein Eigenwertproblem zur Approximation des kritischen Lastparameters gelöst wird. Oft sind nur wenige Zeitverlaufsberechnungen begrenzter Dauer zur hinreichenden Bestimmung der kinetischen Grenzlast erforderlich. Alternativ resultieren Kriterien zur Identifikation der kinetischen Grenzlast aus der Energieverteilung in konservativen Systemen. Ein entsprechendes Kriterium ist mit dem Sattelpunkt-Kriterium nach 
Hoff und Bruce [74] gegeben. Insbesondere bei mehrdimensionalen Systemen ist die genaue Berechnung des Sattelpunktes bzw. kritischen Zustandes für eine adäquate Bewertung der Stabilität entscheidend. Diesem Gedanken folgt das in Kapitel 4 vorgestellte Störenergie-Konzept, mit dem auch ungünstige Störlasten ermittelbar sind.

Die Bewertung der Stabilität von Gleichgewichtszuständen statischer Systeme ist mit dem statischen Stabilitätskriterium möglich. Das Kriterium folgt im Rahmen einer Weggrößenformulierung aus der Linearisierung der Gleichgewichtsbedingungen zu

$$
\mathbf{K}_{T} \Delta \mathbf{v}=\mathbf{0} .
$$

Positive Definitheit der symmetrischen Tangentenmatrix ist Voraussetzung für die Stabilität eines Gleichgewichtszustandes. Positive Semidefinitheit der Tangentenmatrix charakterisiert indifferente Gleichgewichtszustände bzw. singuläre Punkte im Last-Verformungs-Pfad. Im allgemeinen Fall nichtlinearen Systemverhaltens ist eine „,begleitende“ Bewertung der Definitheit erforderlich. Die Bewertung ist mittels der Eigenwerte, Trägheit und Determinante der Tangentenmatrix realisierbar. Die Eigenwerte $\lambda_{i}$ können mit Hilfe des Eigenwertproblems

$$
\left[\mathbf{K}_{T}-\lambda_{i} \mathbf{I}\right] \boldsymbol{\phi}_{i}=\mathbf{0}
$$

berechnet werden. Ist der kleinste Eigenwert identisch null, so ist die Tangentenmatrix positiv semidefinit. Die Art des singulären Punktes wird oft mittels

$$
\boldsymbol{\phi}^{T} \mathbf{p}\left\{\begin{array}{l}
=0: \text { einfacher Verzweigungspunkt } \\
\neq 0: \text { Durchschlagpunkt }
\end{array}\right.
$$

bestimmt. Kriterium (3.9) folgt aus der Diskussion der mit einer Bahnkoordinate parametrisierten Gleichgewichtsbedingungen und ist für die angegebenen singulären Punkte notwendig, aber nicht hinreichend, da auch andere singuläre Punkte vorliegen können [152]. Zur Identifikation der Art eines singulären Punktes sind höhere Ableitungen der Gleichgewichtsbedingungen nach der Bahnkoordinate zu bewerten. Bei der Lösung des Eigenwertproblems (3.8) ist zu beachten, dass mit der inversen Vektoriteration nur die betragsmäßig kleinsten Eigenwerte bestimmt werden können. Der relativ große Aufwand für eine Eigenwertanalyse kann umgangen werden, wenn zur Berechnung des jeweiligen Gleichgewichtszustandes eine $\mathbf{L D L}{ }^{T}$-Zerlegung der Tangentenmatrix erfolgt. In diesem Fall ist die Definitheit der Tangentenmatrix effizient mit der Trägheit oder der Determinante der Diagonalmatrix D bestimmbar [121]. Nullstellen der Determinante sind u.U. nicht problemlos zu ermitteln [20]. Bei Systemen mit großer Anzahl von Freiwerten ist zur Vermeidung numerischer Probleme eine Skalierung der Determinante erforderlich. Eine mögliche Skalierung kann mit der Logarithmus-Funktion erfolgen [152]. Determinante und Trägheit der Tangentenmatrix allein enthalten keine Informationen zur Art eines singulären Punktes. 
Mit dem Current-Stiffness-Parameter $S_{p}$ nach Bergan et al. [11] ist ein weiteres Kriterium zur Bewertung der Stabilität von Gleichgewichtszuständen statischer Systeme gegeben. Der Parameter ist definiert als Quotient aus den Arbeiten, welche eine Referenzbelastung auf den im Prädiktor-Schritt des ersten und aktuellen Lastschrittes berechneten inkrementellen Zustandsgrößen leistet. Für einen Durchschlagpunkt gilt $S_{p}=0$. Verzweigungspunkte sind mit dem Parameter erst in Zusammenhang mit der Determinante der Tangentenmatrix identifizierbar. Vorteilhaft ist der relativ geringe Aufwand zur Berechnung des Current-Stiffness-Parameters.

Zur Identifikation der Stabilitätsgrenze statischer Systeme sind auch Eigenwertprobleme geeignet, die auf der Annahme eines abschnittsweise linearen Zusammenhanges zwischen Belastung und Zustandsgrößen basieren [20, 73]. Ein entsprechendes Eigenwertproblem ist mit

$$
\left[\mathbf{K}_{T}\left(\mathbf{v}_{0}, \mathbf{v}_{0}\right)+\Delta \lambda \mathbf{K}_{0, N L}\left(\mathbf{v}_{0}, \mathbf{v}_{0}\right)\right] \boldsymbol{\phi}=\mathbf{0}
$$

gegeben. Mit dem Inkrement des Eigenwertes wird das Lastniveau der Stabilitätsgrenze bzw. die Beullast approximiert zu

$$
\mathbf{p}_{c r} \approx(1+\Delta \lambda) \mathbf{p}_{0}
$$

Sukzessive Lösung des Eigenwertproblems für weitere Lastniveaus führt zur hinreichend genauen Identifikation der Stabilitätsgrenze. Der Eigenvektor $\phi$ zeigt bei Verzweigungspunkten den Verformungszustand des sekundären Pfades auf und ermöglicht die Anwendung von Pfadwechselprozeduren [118]. Verschiedene lineare und nichtlineare Eigenwertprobleme zur Abschätzung der Stabilitätsgrenze werden in $[20,71]$ erörtert. Die direkte Berechnung der Stabilitätsgrenze gelingt mit erweiterten Gleichungssystemen. Dabei ist das Gleichungssystem zur Berechnung eines Gleichgewichtszustandes um Bedingungen zur Identifikation singulärer Punkte und Normierung des Eigenvektors zu erweitern. Die erweiterten Gleichungssysteme besitzen oft die Dimension 2n+1, sind aber mit nur geringem zusätzlichen Aufwand lösbar [152].

Zur Bewertung der Stabilität von Gleichgewichtszuständen konservativer Systeme ist neben dem statischen auch das energetische Stabilitätskriterium geeignet. Dieses Kriterium setzt die Existenz eines hinreichend stetigen Energiefunktionals voraus und resultiert formal aus der ersten Variation der potentiellen Energie. Die Variation ist sowohl für den indifferenten Gleichgewichtszustand 0 als auch für einen benachbarten nichttrivialen Gleichgewichtszustand 1 identisch null. Für letzteren kann die Variation wie folgt dargestellt werden,

$$
\bar{\delta} \Pi_{1}=\bar{\delta}\left(\Pi_{0}+\frac{1}{2} \delta^{2} \Pi_{0}+\frac{1}{6} \delta^{3} \Pi_{0}+\ldots\right)=0
$$

Bei Vernachlässigung kubischer und höherer Variationen folgt

$$
\bar{\delta} \Pi_{1}=\bar{\delta}\left(\delta^{2} \Pi_{0}\right)=0 \text {. }
$$


Dieses Kriterium wird auch als Trefftz-Kriterium [10] bezeichnet und identifiziert den Gleichgewichtszustand 0 als indifferent, wenn die erste Variation $\bar{\delta}$ einer zweiten Variation $\delta^{2}$ des elastischen Potentials eines Systems zu null wird. Für den Sonderfall $\bar{\delta}=\delta$ ist die Stabilität des Gleichgewichtszustandes anhand der zweiten Variation

$$
\delta^{2} \Pi_{0}\left\{\begin{array}{l}
>0: \text { stabil } \\
=0: \text { indifferent } \\
<0: \text { instabil }
\end{array}\right.
$$

bewertbar. Kriterium (3.14) beschreibt die Energiefläche eines Systems im Bereich des Gleichgewichtszustandes asymptotisch und ermöglicht somit u.U. nur lokal begrenzte Stabilitätsaussagen. Ein stabiler (instabiler) Gleichgewichtszustand ist durch ein zumindest lokales Minimum (Maximum) des elastischen Potentials charakterisiert und bedingt bei einer Störung eine Energiezufuhr (Freisetzung potentieller Energie). Das Kriterium ist nur notwendig, aber nicht hinreichend für die Existenz eines indifferenten Gleichgewichtszustandes [116]. Zur Identifikation der ersten bei Laststeigerung auftretenden Stabilitätsgrenze ist das Kriterium dennoch verwendbar. Koiter [89] verifiziert das Kriterium mit Hilfe thermodynamischer Betrachtungen und zeigt, dass der Verlust der Stabilität bei elastischem Materialverhalten ein isothermer Prozess ist. Bedenken hinsichtlich der uneingeschränkten Gültigkeit des Kriteriums bestehen, da nicht abgesichert ist, dass bei mehrdimensionalen Kontinua von einer positiv definiten zweiten Variation des elastischen Potentials auf die Stabilität eines Gleichgewichtszustandes im Sinne von Ljapunow zu schließen ist [134, 101].

Bei Systemen mit nichtkonservativer statischer Belastung existieren an der Stabilitätsgrenze oftmals keine nichttrivialen Gleichgewichtszustände. In diesen Fällen versagt das statische Stabilitätskriterium und die Stabilität eines Gleichgewichtszustandes ist mit dem kinetischen Stabilitätskriterium zu untersuchen [166]. Diesem Kriterium zufolge ist ein Gleichgewichtszustand stabil, wenn eine Störung eine Bewegung initiiert, die in dessen näheren Umgebung verbleibt. Die Bewertung der Stabilität sollte die Dämpfungseigenschaften eines Systems berücksichtigen und kann mit Ljapunow-Exponenten erfolgen. Systeme mit verformungsabhängiger Belastung sind, sofern bestimmte Anforderungen an Belastung oder Randbedingungen erfüllt werden, als konservativ einstufbar $[131,86]$. Für konservative Systeme führen statisches, energetisches und kinetisches Kriterium auf gleichwertige Stabilitätsaussagen.

Die vorgestellten Kriterien zur Bewertung der Stabilität von Gleichgewichtszuständen konservativer Systeme sind bei mit Weggrößenelementen diskretisierten Systemen definitiv anwendbar. Für die in Abschnitt 2.2 vorgestellte gemischte Formulierung ist Folgendes zu beachten: Ein stabiler Gleichgewichtszustand stellt nicht ein 
Minimum des elastischen Potentials, sondern einen Sattelpunkt dar. Die Tangentenmatrix ist dabei nicht für beliebige, aber für physikalisch sinnvolle Vektoren, d.h. Lösungsvektoren des Gleichungssystems, positiv definit. Bei einer $\mathbf{L D L}^{T}$-Zerlegung der Tangentenmatrix ist Matrix D oft keine Diagonalmatrix. Determinante und Trägheit der Tangentenmatrix und Matrix D sind identisch. Aufgrund der Spannungsfreiwerte existieren für stabile Gleichgewichtszustände negative Eigenwerte der Tangentenmatrix. Daher ist im Rahmen der gemischten Formulierung das Eigenwertproblem (3.8) nicht zur Bewertung der Stabilität von Gleichgewichtszuständen geeignet.

\subsection{Singuläre Punkte}

Singuläre Punkte repräsentieren spezielle und u. U. nicht eindeutige Systemzustände. Bei der Bewertung der Stabilität eines Gleichgewichtszustandes im Sinne von Ljapunow wird die zeitliche Entwicklung einer vorgegebenen Störung der Anfangsbedingungen betrachtet. Mit den Eigenwerten der Tangentenmatrix, welche die zeitliche Entwicklung der Störung beschreibt, kann das Systemverhalten im Bereich des Gleichgewichtszustandes verdeutlicht und dessen Stabilität bewertet werden [93].

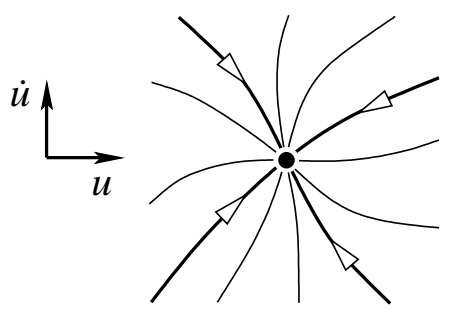

stabiler Knoten

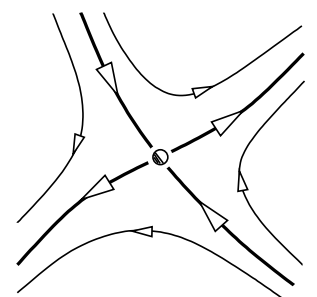

Sattelpunkt

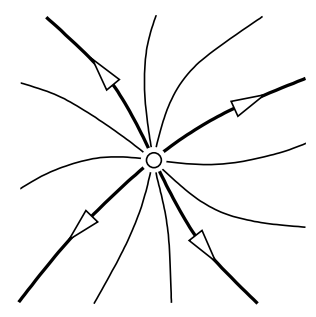

instabiler Knoten

Bild 3.2: Systemverhalten im Bereich singulärer Punkte [94]

Für ein durch Kriechbewegungen charakterisiertes System der Dimension $N=2$ zeigt Bild 3.2 anhand von Trajektorien das mögliche Systemverhalten im Bereich verschiedener singulärer Punkte auf. Im Bereich eines stabilen Knotens streben die Trajektorien zum singulären Punkt. Der Gleichgewichtszustand ist stabil. Für einen Sattelpunkt und einen instabilen Knoten zeigen die Trajektorien die Instabilität des Gleichgewichtszustandes auf. $\mathrm{Zu}$ den singulären Punkten schwingungsfähiger Systeme zählen stabile und instabile Strudel. Für einen stabilen Strudel sind gedämpfte oszillierende Bewegungen um diesen singulären Punkt kennzeichnend. Dagegen können angefachte oszillierende Bewegungen auf einen instabilen Strudel hinweisen. Den Grenzfall zwischen stabilem und instabilem Strudel bilden Wirbelpunkte. Diese Punkte sind für ungedämpfte periodische Bewegungen charakteristisch.

Bei statischen Systemen sind singuläre Punkte im Last-Verformungs-Pfad mittels der Eigenwerte der Tangentenmatrix identifizierbar. Bestimmte singuläre Punkte, die 
Durchschlag- und die einfachen Verzweigungspunkte, kennzeichnen oft das Tragverhalten von Schalentragwerken. Eine ausführliche Diskussion singulärer Punkte und von Kriterien zu deren Charakterisierung erfolgt in [152]. Bild 3.3 zeigt verschiedene singuläre Punkte und den Verlauf des Last-Verformungs-Pfades in deren Bereich.
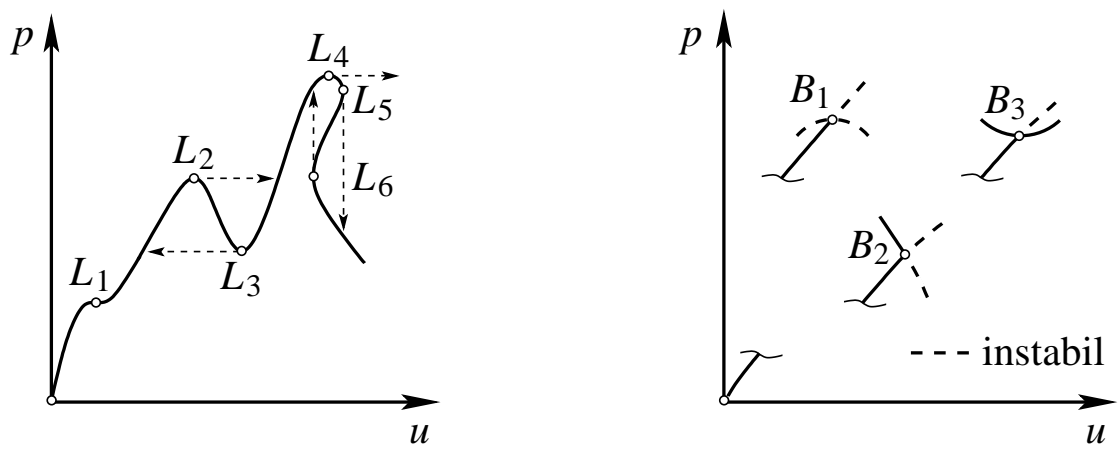

Bild 3.3: Durchschlag- und Verzweigungspunkte

Punkt $L_{1}$ repräsentiert einen speziellen Durchschlagpunkt, der als Sattelpunkt bezeichnet wird. An den durch ein lokales Extremum charakterisierten Durchschlagpunkten $L_{2}-L_{6}$ schlägt das System in einen anderen Gleichgewichtszustand durch. Ein Durchschlag ist lokaler oder globaler Natur. Die Richtung eines Durchschlages ist von der Steuerung des Systems sowie von Niveau und Richtung der Belastung abhängig. Die Durchschlagpunkte $L_{1}-L_{4}$ können bei Last-, die Punkte $L_{5}$ und $L_{6}$ bei Wegsteuerung auftreten. Bei Wegsteuerung stellt $p$ in Bild 3.3 ein Maß für die innere Reaktion des Systems dar. Die Wirkung der Belastung auf die Richtung des Durchschlages wird bei Betrachtung eines Gleichgewichtszustandes im Bereich $L_{3}-L_{4}$ deutlich. Wird ausgehend von diesem Zustand das Lastniveau verringert, so schlägt das System vom Punkt $L_{3}$ in einen Gleichgewichtszustand im Bereich $L_{1}-L_{2}$ durch.

Verzweigungspunkte können mittels Betrachtung des sekundären Last-VerformungsPfades im Verzweigungspunkt klassifiziert werden [88]. Der Verzweigungspunkt $B_{1}$ ist durch ein instabiles symmetrisches und der Verzweigungspunkt $B_{2}$ durch ein unsymmetrisches Verzweigungsverhalten charakterisiert. Derartige Verzweigungspunkte kennzeichnen sehr ungünstiges Systemverhalten, da bereits eine kleine Störung dieser Systemzustände die Tragfähigkeit signifikant verringern kann. Bei unsymmetrischen Verzweigungen ist die Entwicklung der Tragfähigkeit von der Richtung der Störung abhängig. Dagegen erlaubt die stabile symmetrische Verzweigung in Punkt $B_{3}$ die Ausnutzung zusätzlicher Tragreserven. Im Bereich einer Verzweigung ist der sekundäre Pfad bei im Vorbeulbereich rotationssymmetrischen Systemen oft durch eine unsymmetrische Verteilung der Zustandsgrößen gekennzeichnet.

In der Katastrophentheorie werden in allgemeiner Form die topologischen Eigenschaften von Potentialflächen sowie Ursachen und Eigenschaften des Verhaltens von Systemen im Bereich singulärer Punkte analysiert. Mit wenigen Kontrollparametern ist eine Klassifizierung von Instabilitäten bzw. Katastrophen möglich. Die Anzahl 

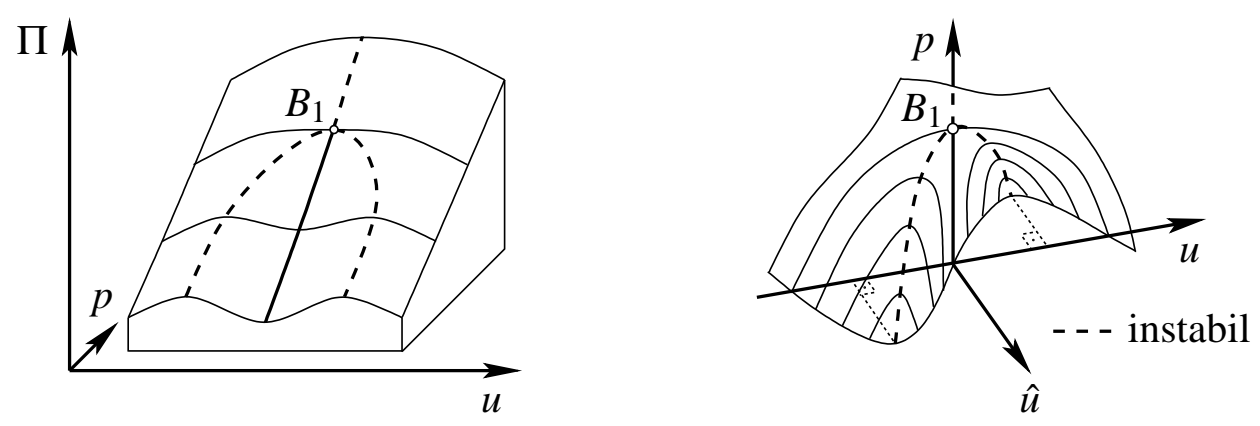

Bild 3.4: Energie- und Gleichgewichtsfläche im Bereich einer Cusp-Katastrophe [10]

beschreibbarer Arten von Katastrophen ist mit der Anzahl unabhängiger Kontrollparameter festgelegt. Ein aktiver Kontrollparameter ist der Lastfaktor, ein passiver die Amplitude $\hat{u}$ einer geometrischen Imperfektion. Zu den Katastrophen, die mit einem Kontrollparameter zu beschreiben sind, zählen Durchschläge und unsymmetrische Verzweigungen [10, 123]. Mit zunehmender Anzahl der Kontrollparameter sind weitere Arten von Katastrophen unterschiedlicher praktischer Relevanz darstellbar, z. B. Cusp-Katastrophen. Eine Cusp-Katastrophe repräsentiert eine stabile symmetrische oder instabile symmetrische Verzweigung. Die Energie- und die Gleichgewichtsfläche im Bereich einer instabilen symmetrischen Verzweigung verdeutlichen die zumindest lokale Komplexität entsprechender Randwertaufgaben, vgl. Bild 3.4.

Singuläre Punkte können einzeln oder in Clustern im primären und in sekundären Last-Verformungs-Pfaden auftreten. Der erste singuläre Punkt im primären Pfad stellt eine Grenze zwischen Vor- und Nachbeulbereich dar. Zur Bewertung des Tragverhaltens von Schalentragwerken ist oftmals nur der Bereich unterhalb dieser Grenze von Bedeutung. An singulären Punkten kann eine Bewegung, der Beulvorgang, initiiert werden, dessen Bewertung weitere Aussagen zum Tragverhalten erlaubt.

\subsection{Imperfektionsempfindlichkeit}

Imperfektionen, allgemeiner Störungen, kennzeichnen reale Systeme. Der Einfluss von Störungen auf das Systemverhalten ist von deren Art und den Eigenschaften des Systems abhängig. Störungen können in numerischer Hinsicht sämtliche Parameter der Modellgleichungen betreffen und sind in anfänglich und nicht anfänglich wirkend zu unterscheiden. Mit dieser Differenzierung können der Zeitpunkt des Auftretens und das Lastniveau beim Auftreten von Störungen näher beschrieben werden. So sind bei statischen Systemen unter anfänglichen Störungen solche Störungen zu verstehen, die bereits bei einer Grundbelastung identisch null vorhanden sind.

Die Anfangs-Nachbeul-Analyse nach Koiter [88] ermöglicht eine Klassifizierung des Verzweigungsverhaltens und die Bewertung des Einflusses kleiner anfänglicher geometrischer Störungen [23]. Derartige Störungen können das Verzweigungsproblem 
eines perfekten Systems in ein Durchschlagproblem des imperfekten Systems überführen und eine Verringerung der Grenzlast bewirken. Dies begründet die Interpretation von Imperfektionsempfindlichkeit als Abhängigkeit der Grenzlast von anfänglichen Störungen. Den Koiter'schen Power-Laws [10] zufolge weisen Systeme im Bereich unsymmetrischer Verzweigungen eine größere Imperfektionsempfindlichkeit auf als in der Nähe instabiler symmetrischer Verzweigungen.

Die Bewertung der Stabilität von Gleichgewichtszuständen im Fall nicht anfänglicher Störungen führt zur Interpretation von Imperfektionsempfindlichkeit als grundzustandspezifische Systemeigenschaft. Dieser Gedanke kommt in dem Stabilitätsgrad nach Leipholz [102] zum Ausdruck und liegt dem Störenergie-Konzept zugrunde. Das Konzept erlaubt quantitative Aussagen zur Imperfektionsempfindlichkeit bei kinetischen und statischen Störungen. Ein dem Stabilitätsgrad inverses Maß ist die von Vielsack et al. definierte Sensitivität [149]. Der Einfluss definierter Entwurfsparameter auf das Systemverhalten ist mittels Parameter-Sensitivitäts-Analyse [84] bewertbar.

Bei Schalentragwerken können insbesondere geometrische Störungen die Grenzlast signifikant beeinflussen. Eßlinger [44] untersucht bei axialgedrückten Kreiszylinderschalen die Wirkung derartiger Störungen und zeigt auf, dass die Störungen zu einer deutlichen Verringerung der im Vorbeulbereich vorhandenen Stützwirkung der Umfangsmembrankräfte und somit zur Verminderung der Grenzlast führen können. Allgemein ist die Intensität der Stützwirkung der Membrankräfte zwar vom Beulfall abhängig, dennoch kann eine merkliche Verringerung der Stützwirkung bereits mit lokal begrenzten Störungen einhergehen. Dabei ist es unwesentlich, ob eine anfängliche geometrische oder nicht anfängliche Laststörung vorliegt. Entscheidend ist, dass beide Störungen auslenkend bzw. destabilisierend wirken können. Ob einzelne oder flächenhafte Laststörungen ungünstiger oder baupraktisch relevanter sind, ist von deren Größe sowie Position abhängig und noch zu beantworten, vgl. [79] sowie Kapitel 8.

Eine im Beulsicherheitsnachweis anzusetzende anfängliche Störung sollte nicht zum Abbau aller im Vorbeulbereich stabilisierend wirkenden Membrankräfte und einer zu konservativen Abschätzung der Grenzlast führen. Da einer bestimmten Grenzlast verschiedene Störungen zugeordnet werden können, sind zur Gewährleistung baupraktisch realistischer Grenzlasten Grenzwerte hinsichtlich Form, Amplitude und Position anfänglicher Störungen bzw. geometrischer Ersatzimperfektionen zu beachten. Letztere können näherungsweise die Wirkung von Störungen unterschiedlicher Art erfassen, sollten aber die Charakteristik des jeweiligen Beulfalles berücksichtigen. Für symmetrische Systeme ist oft der Ansatz unsymmetrischer Störungen zu empfehlen. Intuitiv gewählte und beuleigenformaffine Störungen werden nicht in allen Fällen das ungünstigste Verhalten eines Systems aufzeigen. 


\section{Störenergie-Konzept}

Das Störenergie-Konzept nach Kröplin et al. [96] ermöglicht die Bewertung der Imperfektionsempfindlichkeit von Schalentragwerken mit Hilfe einer energetischen Größe, der Störenergie. Hiermit sind die Grenzlasten verschiedener Beulfälle realistisch beschreibbar und mit den Regelwerken zum Beulsicherheitsnachweis zu vergleichen.

\subsection{Einführung}

Das Tragverhalten von Schalentragwerken wird von Imperfektionen, allgemeiner Störungen, der Systemparameter beeinflusst. $\mathrm{Zu}$ diesen Parametern zählen Kennwerte der Belastung, Geometrie und Randbedingungen sowie Materialparameter. Bild 4.1 zeigt das Last-Verformungs-Verhalten einer axialgedrückten Kreiszylinderschale auf.

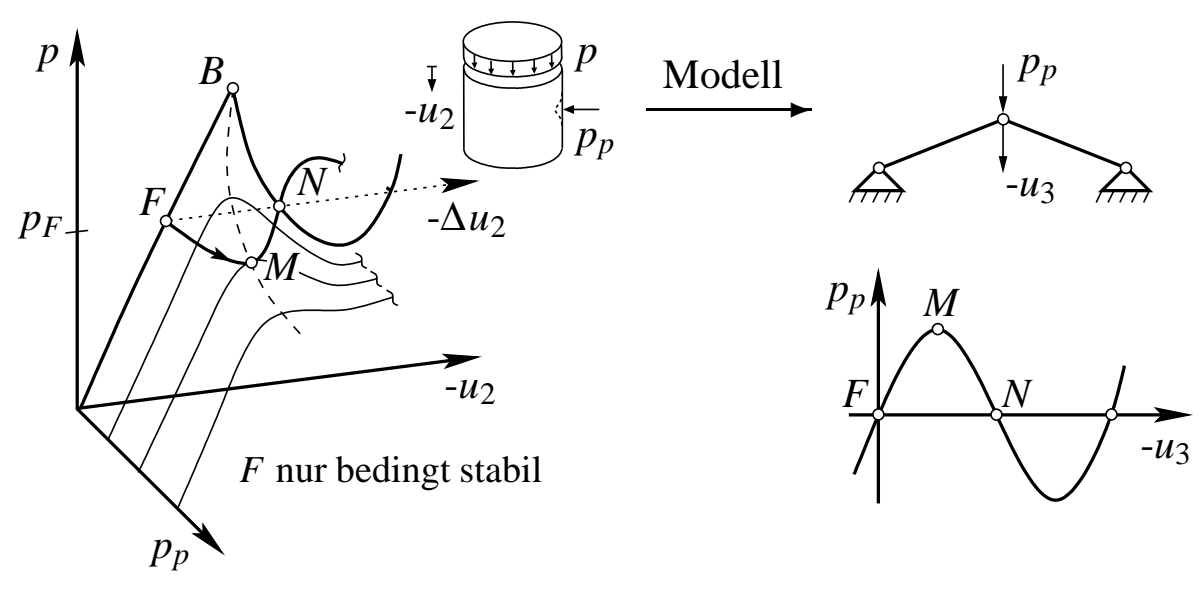

Bild 4.1: Last-Verformungs-Verhalten eines Schalen- und Stabtragwerkes [34]

Die Volllinie in der $p-u_{2}$-Ebene repräsentiert das Tragverhalten der perfekten axialgedrückten Kreiszylinderschale. Im Vorbeulbereich ist dieses durch einen unbedingt und einen bedingt stabilen Bereich charakterisiert. Eine statische Störlast $p_{p}$, die während des gesamten Belastungsvorganges mit konstantem Betrag und orthogonal zur Grundbelastung $p$ wirkt, verringert das Maximum $M$ der inneren Reaktion und überführt das Verzweigungsproblem der perfekten Kreiszylinderschale in ein Durchschlagproblem. Die Analyse des Tragverhaltens für das Lastniveau $p_{F}$ verdeutlicht, dass die Kreiszylinderschale bei einer hinreichend großen Störlast, die nur 
auf diesem Lastniveau wirkt, vom stabilen Grundzustand $F$ im Vorbeulbereich in den kritischen Zustand $M$ überführt wird. Eine Steigerung der Störlast bewirkt ein Durchschlagen der Kreiszylinderschale in den Nachbeulbereich, wo nach Abklingen der initiierten Systembewegung ein stabiler Gleichgewichtszustand erreicht wird.

Grundgedanke des Störenergie-Konzeptes ist die Berechnung des Minimums an Formänderungsenergie, das erforderlich ist, um ein Schalentragwerk von einem stabilen Grundzustand in einen kritischen Zustand zu überführen. Diese Formänderungsenergie wird im Weiteren als Störenergie $\Pi_{c r}$ bezeichnet. Die Berechnung der Störenergie erfordert die Identifikation des kritischen Zustandes. Das in Bild 4.1 dargestellte Stabtragwerk verdeutlicht als Modell für das lokale Tragverhalten den lokalen Charakter des Beginns des Beulvorganges eines Schalentragwerkes und den kritischen Zustand in Abhängigkeit von der Art der Störlast. Für eine statische Störlast ist aufgrund der verschwindenden Tangentensteifigkeit der Zustand $M$ der kritische Zustand. Bei einer kurzzeitigen kinetischen Störlast repräsentiert infolge der verschwindenden inneren Reaktion bzw. Sekantensteifigkeit der Zustand $N$ den kritischen Zustand.

Die Bewertung der Imperfektionsempfindlichkeit und der Grenzlasten von Schalentragwerken beginnt mit der Identifikation des ersten singulären Punktes im primären Last-Verformungs-Pfad. Hierzu sind verschiedene der in Abschnitt 3.4 vorgestellten Stabilitätskriterien geeignet. Anschließend werden für unterschiedliche Lastniveaus unterhalb des singulären Punktes und oberhalb des Nachbeulminimums die kritischen Zustände $M$ und $N$ identifiziert. Mit Hilfe der zugehörigen Störenergie können die statische und die kinetische Grenzlast ermittelt werden. Die Identifikation kritischer Zustände und die Ermittlung der Grenzlasten werden nachfolgend diskutiert.

\subsection{Identifikation kritischer Zustände}

In mathematischer Hinsicht ist der Grundgedanke des Störenergie-Konzeptes als Optimierungsproblem interpretierbar. Dieses besitzt das Ziel, den für einen Grundzustand kritischen Zustand in Abhängigkeit von der Art der Störlast zu identifizieren. Oftmals existiert aber eine Vielzahl kritischer Zustände. Unter der Annahme, dass die jeweils energieärmere Lösung die wahrscheinlichere darstellt, ist für einen Grundzustand der in energetischer Hinsicht benachbarte kritische Zustand zu ermitteln.

\subsubsection{Modell bei elastischem Materialverhalten}

Im Rahmen der gewählten gemischten Formulierung ist bei elastischem Materialverhalten die Zielfunktion $f$ des Optimierungsproblems definiert zu

$$
f\left(\mathbf{z}_{F}, \Delta \mathbf{z}\right)=\Pi_{c r} \rightarrow \min .
$$


Die Zielfunktion fordert die Minimierung der Störenergie (4.15) und ist von den Zustandsgrößen $\mathbf{z}_{F}$ des Grundzustandes und der Änderung $\Delta \mathbf{z}$ der Zustandsgrößen beim Übergang in einen kritischen Zustand abhängig. Die Lösung des Optimierungsproblems (4.1) erfolgt anhand der nachfolgend unter Berücksichtigung der Art der Störlast hergeleiteten Eigenwertprobleme zur Identifikation kritischer Zustände.

Kinetische Störlast. Im Fall einer kurzzeitigen kinetischen Störlast ist ein kritischer Zustand durch eine bezüglich des Grundzustandes $F$ verschwindende Sekantensteifigkeit charakterisiert. Dies entspricht dem Verschwinden der ersten Variation des inkrementellen Potentials, sofern die inkrementelle Belastung identisch null ist,

$$
\delta \Delta \Pi\left(\mathbf{z}_{F}, \Delta \mathbf{z}\right)=\delta \Delta \mathbf{z}^{T}\left[\mathbf{A}_{T}\left(\mathbf{z}_{F}\right)+\frac{1}{2} \mathbf{A}_{N L}(\Delta \mathbf{z})\right] \Delta \mathbf{z}=0
$$

Aus der ersten Variation folgt mit $\Delta \mathbf{z}=\lambda \boldsymbol{\phi}$ das Eigenwertproblem

$$
\left[\mathbf{A}_{T}\left(\mathbf{z}_{F}\right)+\lambda \frac{1}{2} \mathbf{A}_{N L}(\boldsymbol{\phi})\right] \boldsymbol{\phi}=\mathbf{0}
$$

mit dem kritische Zustände $N$ identifizierbar sind. Nach Ermittlung einer nichttrivialen Lösung $\Delta \mathbf{z}=\Delta \mathbf{z}_{c r, N}$ ist zu überprüfen, ob mit der Lösung ein instabiler Gleichgewichtszustand bzw. ein zumindest lokales Maximum des elastischen Potentials beschrieben wird, vgl. Bild 4.2 rechts. Die Überprüfung kann anhand der Definitheit der Tangentenmatrix $\mathbf{A}_{T}\left(\mathbf{z}_{F}+\Delta \mathbf{z}\right)$ bezüglich der Lösung erfolgen.

Statische Störlast. Im Fall einer statischen Störlast ist ein kritischer Zustand durch eine bezüglich des Grundzustandes $F$ verschwindende Tangentensteifigkeit bzw. verschwindende zweite Variation des inkrementellen Potentials charakterisiert,

$$
\delta^{2} \Delta \Pi\left(\mathbf{z}_{F}, \Delta \mathbf{z}\right)=\delta \Delta \mathbf{z}^{T}\left[\mathbf{A}_{T}\left(\mathbf{z}_{F}\right)+\mathbf{A}_{N L}(\Delta \mathbf{z})\right] \delta \Delta \mathbf{z}=0 .
$$

Die zweite Variation folgt mit $\Delta \mathbf{z}=\lambda \phi$ und $\delta \Delta \mathbf{z}=\delta \lambda \phi+\lambda \delta \phi$ sowie unter Berücksichtigung von $\mathbf{A}_{T}\left(\mathbf{z}_{F}\right)+\mathbf{A}_{N L}(\lambda \boldsymbol{\phi})=\mathbf{A}_{T} \mathrm{zu}$

$$
\lambda^{2} \delta \boldsymbol{\phi}^{T}\left\{\mathbf{A}_{T} \delta \boldsymbol{\phi}\right\}+2 \lambda \delta \lambda \boldsymbol{\phi}^{T}\left\{\mathbf{A}_{T} \delta \boldsymbol{\phi}\right\}+\delta \lambda^{2} \boldsymbol{\phi}^{T}\left\{\mathbf{A}_{T} \boldsymbol{\phi}\right\}=0
$$

Die ersten beiden Terme der Gleichung beinhalten das statische Stabilitätskriterium und sind für kritische Zustände $M$ identisch null, da diese singuläre Zustände repräsentieren. Somit sind kritische Zustände anhand des Eigenwertproblems

$$
\left[\mathbf{A}_{T}\left(\mathbf{z}_{F}\right)+\lambda \mathbf{A}_{N L}(\boldsymbol{\phi})\right] \boldsymbol{\phi}=\mathbf{0}
$$

identifizierbar. Das Eigenwertproblem lässt erkennen, dass kritische Zustände $M$ Durchschlagpunkte darstellen. Die Lösung $\Delta \mathbf{z}=\Delta \mathbf{z}_{c r, M}$ des Eigenwertproblems beschreibt einen Systemzustand mit verschwindender Krümmung der Energiefläche, 
vgl. Bild 4.2 rechts. Auch wenn ein kritischer Zustand ohne inkrementelle Belastung ermittelbar ist, so ist dieser nur infolge einer entsprechenden Störung der Grundbelastung zu erreichen. Die einem kritischen Zustand $M$ zugehörige inkrementelle Belastung wird mittels der ersten Variation des inkrementellen Potentials zu

$$
\mathbf{p}_{p}=\Delta \mathbf{p}=\left[\mathbf{A}_{T}\left(\mathbf{z}_{F}\right)+\frac{1}{2} \mathbf{A}_{N L}(\Delta \mathbf{z})\right] \Delta \mathbf{z}=-\frac{1}{2} \mathbf{A}_{N L}(\Delta \mathbf{z}) \Delta \mathbf{z}
$$

berechnet und als Störlast bezeichnet. Die Störlast ist quadratisch von den inkrementellen Zustandsgrößen abhängig und umfasst Kräfte und Membranverzerrungen. Ferner ist die Störlast, die nur in vektorieller Form vorliegt, oft orthogonal zu dem Vektor, der die zur Grundbelastung arbeitsäquivalenten Knoteneinwirkungen enthält. Somit ist zu erwarten, dass infolge der Störlast ein im Vergleich zum primären Tragverhalten unterschiedliches Systemverhalten angeregt wird.

Weitere Arten von Störungen. Der Identifikation kritischer Zustände liegen charakteristische Eigenschaften des inkrementellen elastischen Potentials zugrunde. Dieses ist von der Belastung und anderen Parametern wie der Wanddicke abhängig. Somit könnte bei der Formulierung des inkrementellen Potentials formal eine Änderung der Wanddicke berücksichtigt werden. Die Änderung ist als eine lastniveauspezifische und eventuell räumlich begrenzte Störung der Steifigkeit eines Systems zu interpretieren und im Rahmen dieser Arbeit als statisch wirkend anzusehen. Die Identifikation eines kritischen Zustandes ist aber nur bei einer definierten Störung der Wanddicke möglich. Eine ungünstige Störung der Wanddicke im Sinne der Störlast (4.7) ist nicht möglich. Dies gilt auch für die Störung anderer Parameter, mit denen die Formänderungsenergie eines Systems zu beschreiben ist. Die Anwendung des StörenergieKonzeptes hinsichtlich der lastniveauspezifischen Störung der Wanddicke und des Bettungsmoduls wird in Abschnitt 4.4 diskutiert.

Lösung der Eigenwertprobleme. Die für den Fall einer kinetischen und den Fall einer statischen Störlast hergeleiteten Eigenwertprobleme zur Identifikation kritischer Zustände können zusammenfassend in der Form

$$
\left[\mathbf{A}_{T}\left(\mathbf{z}_{F}\right)+\lambda \xi \mathbf{A}_{N L}(\boldsymbol{\phi})\right] \boldsymbol{\phi}=\mathbf{0}, \text { mit } \xi=\frac{1}{2} \text { bzw. 1,0 }
$$

dargestellt werden, wobei der Koeffizient $\xi$ von der Art der Störlast abhängig ist. Beide Eigenwertprobleme weisen eine nichtlineare Abhängigkeit vom Eigenvektor auf und sind mit den in Abschnitt 2.5.3 vorgestellten Verfahren lösbar. Die Lösungen $\Delta \mathbf{z}_{c r, N}$ und $\Delta \mathbf{z}_{c r, M}$ beschreiben, wie in Bild 4.2 aufgezeigt, im $n$-dimensionalen Raum der Zustandsgrößen sowohl die Richtung als auch den Abstand zwischen einem Grundzustand $F$ und dem jeweiligen kritischen Zustand. Die Richtung ist bei kinetischer und statischer Störlast identisch. Der Abstand ist im Rahmen der gewählten gemischten Formulierung bei einer kinetischen Störlast doppelt so groß wie bei 

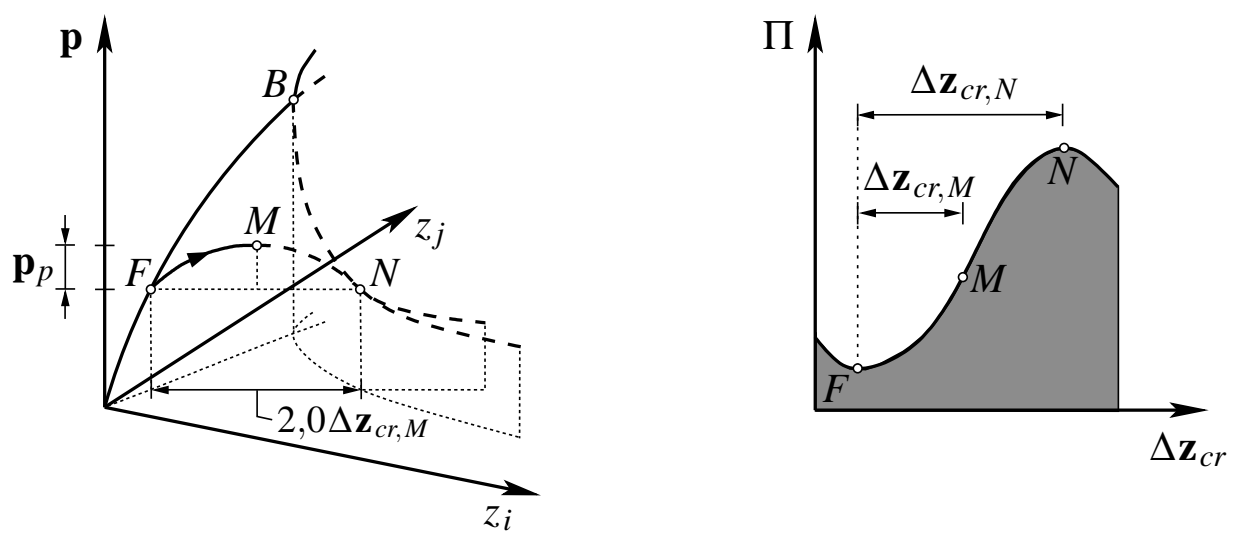

Bild 4.2: Richtung und Abstand zwischen Grundzustand und kritischen Zuständen

einer statischen Störlast. Da die Eigenwertprobleme bezüglich eines Grundzustandes formuliert sind, besteht kein unmittelbarer Zusammenhang zwischen den inkrementellen Zustandsgrößen $\Delta \mathbf{z}_{c r}$ und einer anfänglichen Störung. Für die Bewertung der Imperfektionsempfindlichkeit ist das Eigenpaar maßgebend, welches auf das Minimum an Störenergie führt. Um die Ermittlung des maßgebenden Eigenpaares zu gewährleisten, sind die Eigenwertprobleme für verschiedene Startvektoren zu lösen.

Zur Anwendung des Modells. Die Effizienz der Verfahren zur Lösung der Eigenwertprobleme (4.8) ist von dem Tragverhalten der Schalentragwerke und der Wahl des Startvektors abhängig. Bei Systemen mit großer Imperfektionsempfindlichkeit ist für hohe und mittlere Lastniveaus der Abstand zwischen Grundzustand und kritischem Zustand relativ klein. Die Eigenwertprobleme sind problemlos zu lösen. Für Lastniveaus nahe dem Nachbeulminimum und bei Systemen mit geringer Imperfektionsempfindlichkeit ist der Abstand zwischen Grundzustand und kritischem Zustand relativ groß. Die Lösung der Eigenwertprobleme ist numerisch aufwendig. Startvektoren zur Lösung der Eigenwertprobleme (4.8) sind in Abhängigkeit vom Tragverhalten des zu analysierenden Systems festzulegen. Bei Systemen mit großer Imperfektionsempfindlichkeit bzw. kurzwelliger Beuleigenform genügt oft die Vorgabe einer Verschiebung in Richtung der Schalennormalen [144]. Bei Systemen mit geringer Imperfektionsempfindlichkeit bzw. langwelliger Beuleigenform kann die Wahl eines dem Beuleigenvektor bereichsweise ähnlichen Startvektors vorteilhaft sein.

Konvergenzprobleme während der Lösung der Eigenwertprobleme treten bei Annäherung verschiedener Pfade im Nachbeulbereich auf, bewirken ein Oszillieren im iterativen Lösungsprozess und verhindern die eindeutige Identifikation eines kritischen Zustandes. In diesem Fall ist die Störanalyse für ein geringfügig geändertes Lastniveau fortzuführen. Eine Alternative hierzu bietet sich bei Systemen mit elastischem Materialverhalten an. Dabei kann ausgehend vom letzten ermittelten kritischen $\mathrm{Zu}$ stand $N$ der Nachbarpfad sukzessive durchlaufen und für verschiedene Lastniveaus anhand der Differenz der Zustandsgrößen zwischen Grundzustand und Nachbarzu- 
stand jeweils ein kritischer Zustand $M$ identifiziert werden. Konvergenzprobleme sind auch im unmittelbaren Bereich des ersten singulären Punktes zu erwarten.

Zur Bewertung der Imperfektionsempfindlichkeit von Grundzuständen weggesteuerter Systeme kann die Wirkung der eingeprägten Weggrößen mit der MultiplikatorenMethode nach Lagrange im inkrementellen elastischen Potential und somit auch in den Bedingungen zur Identifikation kritischer Zustände erfasst werden. Die Richtung des Überganges von einem Grundzustand in einen kritischen Zustand differiert bei weggesteuerten Systemen von der bei lastgesteuerten Systemen, vgl. Abschnitt 3.5. Grund hierfür ist die systemspezifische Topologie der Energiefläche. Der Übergang vom Grundzustand in den kritischen Zustand kann unabhängig von der Steuerung eines Systems dennoch nur von einer nicht anfänglichen Störlast initiiert werden.

\subsubsection{Modell bei elastisch-plastischem Materialverhalten}

Bei elastisch-plastischem Materialverhalten liegt der Identifikation eines kritischen Zustandes $M$ das inkrementelle Potential (2.43) zugrunde. Das Potential ermöglicht für ein Inkrement $\Delta \mathbf{z}$ der Zustandsgrößen die Berechnung des Zuwachses an Formänderungsenergie und Dissipationsarbeit. Eine verschwindende zweite Variation des Potentials charakterisiert wie im Fall elastischen Materialverhaltens einen kritischen Zustand $M$. Das zugehörige Eigenwertproblem kann aufgrund der beim Übergang vom Grundzustand $F$ in den kritischen Zustand $M$ fortschreitenden Plastifizierung des Schalenkontinuums nicht direkt gelöst werden. Vielmehr ist ein inkrementelles Vorgehen erforderlich. Ein entsprechender Lösungsansatz wird von Spohr [139] vorgestellt. Die Grundidee dieses Lösungsansatzes ist Bild 4.3 aufgezeigt.
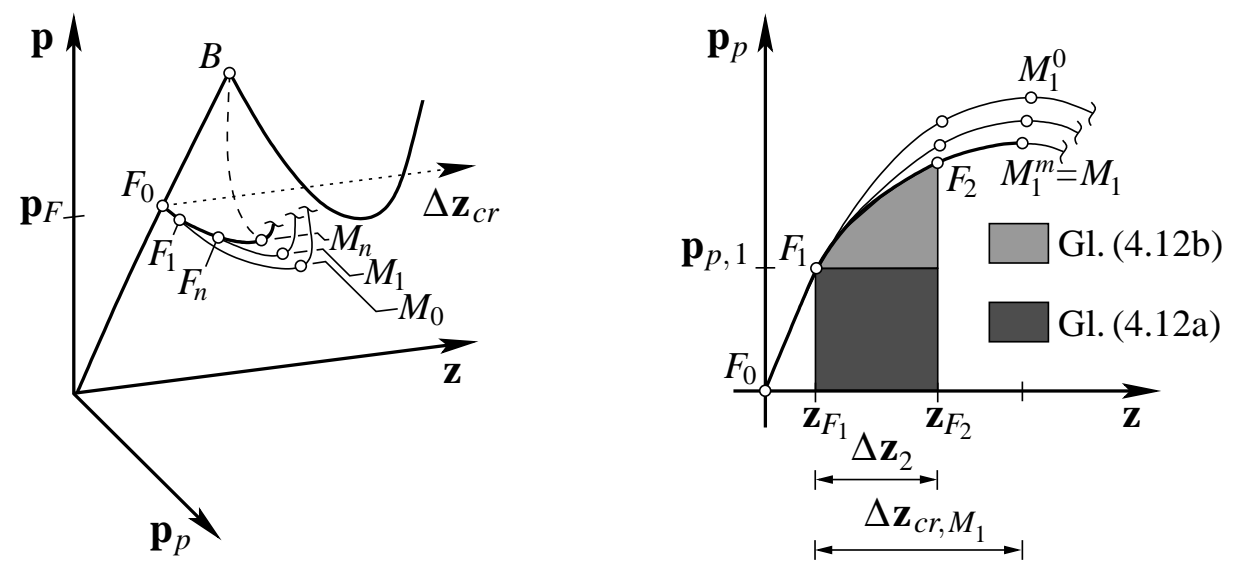

Bild 4.3: Inkrementelle Berechnung des kritischen Zustandes $M[139,87]$

Dem Lösungsansatz folgend ist ausgehend vom Systemzustand $F_{k}=F_{0}$ mit den $\mathrm{Zu}$ standsgrößen $\mathbf{z}_{F_{k}}, \Delta \mathbf{z}_{k+1}=\mathbf{0}$ die Tangentenmatrix

$$
\tilde{\mathbf{A}}_{T}\left(\mathbf{z}_{F_{k}}, \Delta \mathbf{z}_{k+1}\right)=\mathbf{A}_{L}+\mathbf{A}_{N L}\left(\mathbf{z}_{F_{k}}\right)+\mathbf{A}_{T, P}\left(\mathbf{z}_{F_{k}}, \Delta \mathbf{z}_{k+1}\right)
$$


zu berechnen und das Eigenwertproblem

$$
\left[\tilde{\mathbf{A}}_{T}\left(\mathbf{z}_{F_{k}}, \Delta \mathbf{z}_{k+1}\right)+\lambda \mathbf{A}_{N L}(\boldsymbol{\phi})\right] \boldsymbol{\phi}=\mathbf{0}
$$

zu lösen. Mit der Lösung $\Delta \mathbf{z}_{c r, M_{k}^{0}}=\lambda \boldsymbol{\phi}$ können die dem kritischen Zustand zugehörigen inkrementellen Zustandsgrößen nur angenähert werden, da die beim Übergang in den kritischen Zustand fortschreitende Plastifizierung des Schalenkontinuums nicht berücksichtigt wird. Als Inkrement in Richtung des kritischen Zustandes wird daher der mit dem Parameter $\eta=\eta_{\text {min }}^{0}$ nach Abschnitt 2.3 abgeminderte Anteil $\Delta \mathbf{z}_{k+1}=$ $\eta \Delta \mathbf{z}_{c r, M_{k}^{0}}$ angesetzt. Mit diesem Anteil ist die Tangentenmatrix (4.9) im Systemzustand $F_{k}$ zu aktualisieren und mit Eigenwertproblem (4.10) eine verbesserte Approximation der inkrementellen Zustandsgrößen zu ermitteln. Sukzessive Korrektur der Tangentenmatrix und Lösung des Eigenwertproblems führen auf die inkrementellen Zustandsgrößen $\Delta \mathbf{z}_{k+1}=\eta \Delta \mathbf{z}_{c r, M_{k}^{m}}$, die einen neuen Systemzustand repräsentieren. Die Berechnung des inkrementellen Zuwachses der Störlast erfolgt dann mit

$$
\Delta \mathbf{p}_{p, k+1}=\left[\mathbf{A}_{T}\left(\mathbf{z}_{F_{k}}\right)+\frac{1}{2} \mathbf{A}_{N L}\left(\Delta \mathbf{z}_{k+1}\right)\right] \Delta \mathbf{z}_{k+1}+\mathbf{A}_{S, P}\left(\mathbf{z}_{F_{k}}, \Delta \mathbf{z}_{k+1}\right) \Delta \mathbf{z}_{k+1} .
$$

Der Zuwachs der Störlast besteht formal aus zwei Anteilen. Der erste Anteil enthält Kräfte sowie elastische und Gesamtverzerrungen, der zweite Anteil plastische Verzerrungen. Unter Berücksichtigung des Zuwachses der Störlast sind Formänderungsenergie und Dissipationsarbeit im Inkrement mit

$$
\begin{aligned}
\Delta \Pi_{c r, M}^{k+1} & =\Delta \mathbf{z}_{k+1}^{T} \sum_{j=0}^{k} \Delta \mathbf{p}_{p, j} \\
& +\Delta \mathbf{z}_{k+1}^{T}\left[\frac{1}{2} \mathbf{A}_{T}\left(\mathbf{z}_{F_{k}}\right)+\frac{1}{6} \mathbf{A}_{N L}\left(\Delta \mathbf{z}_{k+1}\right)\right] \Delta \mathbf{z}_{k+1}
\end{aligned}
$$

berechenbar. Der erste Term in dieser Gleichung erfasst die Arbeit der dem Systemzustand $F_{k}$ zugehörigen Störlast auf den inkrementellen Zustandsgrößen, vgl. Bild 4.3 rechts. Der zweite Term repräsentiert wie der erste Formänderungsenergie und Dissipationsarbeit, resultiert aber nur aus den aktuellen inkrementellen Zustandsgrößen. Nach Aktualisierung der Zustandsgrößen und der Störlast,

$$
\mathbf{z}_{F_{k+1}}=\mathbf{z}_{F_{k}}+\Delta \mathbf{z}_{k+1} \quad \text { und } \quad \mathbf{p}_{p, k+1}=\mathbf{p}_{p, k}+\Delta \mathbf{p}_{p, k+1},
$$

ist das Eigenwertproblem (4.10) für weitere Systemzustände und weitere Inkremente zu lösen, bis der kritische Zustand hinreichend genau ermittelt ist. Aus der Summe aller inkrementellen Zustandsgrößen folgt der Abstand zwischen Grundzustand und kritischem Zustand. Anzahl und Größe der Inkremente sind von der Plastifizierung des Schalenkontinuums abhängig. Zwischen zwei Systemzuständen kann sich die Richtung der Störlast ändern. Es ist zu beachten, dass die Symmetrisierung der Tangentenmatrix (2.44) die Identifikation des kritischen Zustandes beeinflussen kann. 
Zur Anwendung des Modells. Ergänzend zu den Ausführungen in Abschnitt 4.2.1 ist zu erwähnen, dass als Startvektor zur Lösung des Eigenwertproblems (4.10) die Lösung des entsprechenden Eigenwertproblems (4.8) geeignet ist, sofern diese den kritischen Zustand hinreichend genau beschreibt. Dabei ist die Anwendbarkeit des Lösungsansatzes nach Spohr [139] aber auf Lastniveaus oberhalb des im Fall elastischen Materialverhaltens ermittelten Nachbeulminimums begrenzt. Konvergenzprobleme bei Lösung des Eigenwertproblems (4.10) sind bei ausgeprägter Plastifizierung des Schalenkontinuums in der Nähe des kritischen Zustandes zu erwarten.

\subsection{Grundenergie, Störenergie und Grenzlasten}

Die einem System inhärente Formänderungsenergie ermöglicht die Definition geeigneter Maße zur Beschreibung der Empfindlichkeit des Systems gegenüber Störungen sowie zur Identifikation der statischen und der kinetischen Grenzlast.

\subsubsection{Grundenergie und Störenergie}

Für elastisches Materialverhalten ist die bei Erreichen eines Grundzustandes im System gespeicherte Formänderungsenergie identisch der Summe aller entsprechenden inkrementellen Energiezuwächse und im Rahmen der gemischten Formulierung mit

$$
\Pi_{F}=\sum_{i=1}^{F} \Delta \Pi_{s t r, i}, \Delta \Pi_{s t r}=\underbrace{\Delta \mathbf{z}^{T} \mathbf{p}_{0}}_{1}+\underbrace{\frac{1}{2} \Delta \mathbf{z}^{T} \mathbf{A}_{T}\left(\mathbf{z}_{0}\right) \Delta \mathbf{z}}_{2}+\underbrace{\frac{1}{6} \Delta \mathbf{z}^{T} \mathbf{A}_{N L}(\Delta \mathbf{z}) \Delta \mathbf{z}}_{3},
$$

zu berechnen. Die Formänderungsenergie $\Pi_{F}$ wird als Grundenergie bezeichnet und besteht für ein Inkrement $\Delta \mathbf{z}$ der Zustandsgrößen aus drei Anteilen, vgl. Bild 4.4 links. Für elastisch-plastisches Materialverhalten ist die Grundenergie um die bis zum Erreichen des Grundzustandes verrichtete Dissipationsarbeit zu erweitern [139].
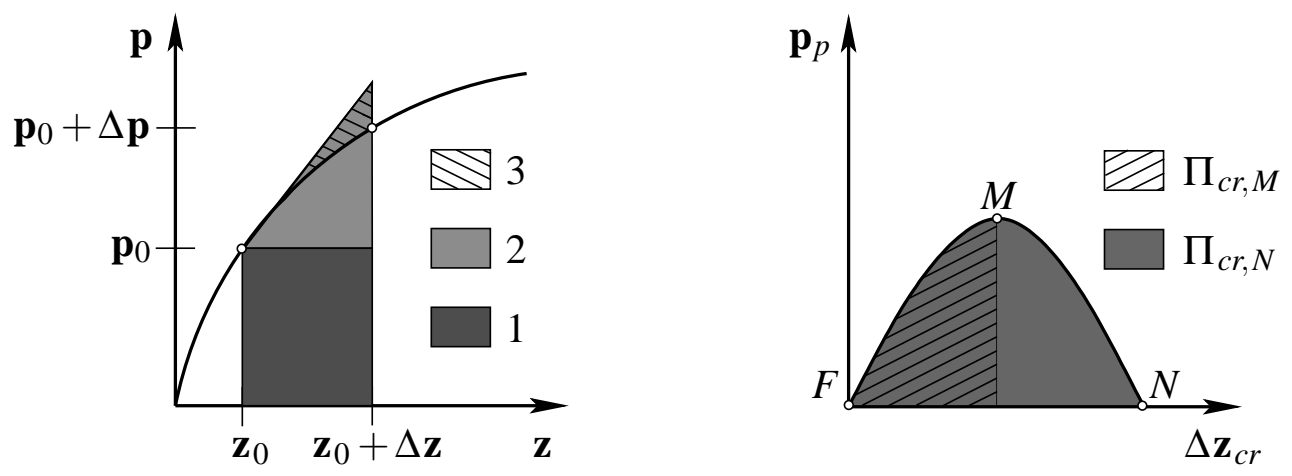

Bild 4.4: Formänderungsenergie im Inkrement sowie Störenergien

Die Störenergie ist bei elastischem Materialverhalten als die Differenz an Formänderungsenergie zwischen Grundzustand $F$ und kritischem Zustand $M$ bzw. $N$ definiert, 
die aus dem Wirken der Störlast gemäß Gl. (4.7) auf der zugehörigen Änderung $\Delta \mathbf{z}_{c r}$ der Zustandsgrößen resultiert. Somit ist, wie Bild 4.4 rechts zeigt, die Störenergie entsprechend dem zweiten und dritten Anteil der Grundenergie (4.14) zu berechnen mit

$$
\begin{aligned}
\Pi_{c r} & =\frac{1}{2} \Delta \mathbf{z}_{c r}^{T} \mathbf{A}_{T}\left(\mathbf{z}_{F}\right) \Delta \mathbf{z}_{c r}+\frac{1}{6} \Delta \mathbf{z}_{c r}^{T} \mathbf{A}_{N L}\left(\Delta \mathbf{z}_{c r}\right) \Delta \mathbf{z}_{c r} \\
& =\frac{1}{2} \Delta \mathbf{z}_{c r}^{T} \mathbf{A}_{L} \Delta \mathbf{z}_{c r}+\frac{1}{2} \Delta \mathbf{z}_{c r}^{T} \mathbf{A}_{N L}\left(\mathbf{z}_{F}\right) \Delta \mathbf{z}_{c r}+\frac{1}{6} \Delta \mathbf{z}_{c r}^{T} \mathbf{A}_{N L}\left(\Delta \mathbf{z}_{c r}\right) \Delta \mathbf{z}_{c r} .
\end{aligned}
$$

Die Störenergie ist ein Maß für die Widerstandsfähigkeit eines Systems gegenüber Störungen eines Grundzustandes und besteht entsprechend Gl. (4.15a) aus zwei Anteilen. Der erste Anteil enthält Membran- und Biegeenergie, der zweite nur Membranenergie. Zur Bewertung der Zusammensetzung der Störenergie wird der erste Anteil in einen vom Lastniveau unabhängigen bzw. abhängigen Anteil getrennt. Der Aufwand zur Berechnung der Grund- und Störenergie kann mit der Analyse des Tragverhaltens an einem Teilsystem verringert werden. Die Größe des Teilsystems ist dabei so zu wählen, dass das Tragverhalten des Gesamtsystems im Bereich der Störlast hinreichend genau wiedergegeben wird. Die an dem Teilsystem ermittelten Formänderungsenergien sind in Abhängigkeit von dessen Größe und der räumlichen Verteilung der Änderung der Zustandsgrößen infolge Störlast zu vervielfachen [144].

Im Fall einer statischen bzw. kinetischen Störlast ist die Störenergie unter Berücksichtigung der Normierung (2.60) des Eigenvektors gleichwertig zu Gl. (4.15) mit

$$
\Pi_{c r, M}=\frac{1}{3}\left(\lambda_{M}\right)^{3} \quad \text { bzw. } \quad \Pi_{c r, N}=\frac{1}{6}\left(\lambda_{N}\right)^{3}
$$

zu berechnen. Die Störenergie ist in beiden Fällen eine Funktion des zugehörigen Eigenwertes. Mit den Gln. (4.16) kann bei gleichzeitiger Auswertung der Gl. (4.15) die Konvergenz der Verfahren zur Lösung der Eigenwertprobleme (4.8) bewertet werden. Zwischen den Gln. (4.16) besteht die in Bild 4.4 rechts erkennbare Relation

$$
\Pi_{c r, M}=0,5 \Pi_{c r, N} .
$$

Die Relation gilt nur in der gewählten gemischten Formulierung und bei elastischem Materialverhalten. Die Relation ermöglicht mit der Lösung nur eines Eigenwertproblems die Bewertung der Stabilität eines Grundzustandes im Fall einer statischen und einer kinetischen Störlast. Bei elastisch-plastischem Materialverhalten ist die Störenergie inkrementell und unter Berücksichtigung der Dissipationsarbeit mit

$$
\Pi_{c r, M}=\sum_{k=1}^{n_{I n k}} \Delta \Pi_{c r, M}^{k}
$$

zu ermitteln, wobei der inkrementelle Zuwachs $\Delta \Pi_{c r, M}^{k}$ aus Gl. (4.12) folgt. Die Störenergie wird i.d.R. für perfekte Schalentragwerke berechnet und soll bei der Identi- 
fikation der statischen Grenzlast den Einfluss verschiedener realer Störungen in integraler Form erfassen. Ferner erlaubt die Störenergie die Bewertung der statischen und der kinetischen Grenzlast mit einem energetisch äquivalenten Einfluss von Störungen.

\subsubsection{Normierung der Störenergie}

Mit der Störenergie $\Pi_{c r, M}$ ist die Empfindlichkeit verschiedener Grundzustände gegenüber statischen Störungen quantitativ bewertbar. Der anschauliche Vergleich der Imperfektionsempfindlichkeit und der statischen Grenzlast unterschiedlicher Beulfälle mit Hilfe eines energetischen Wertes bedingen aber eine Normierung der Störenergie. Wagenhuber [151] schlägt eine Normierung der Störenergie mittels der zum ersten singulären Zustand eines Systems korrespondierenden Grundenergie vor. Die Grundenergie ist aber für das gesamte System zu ermitteln, womit eine äquivalente Normierung der Störenergie bei lokalen und globalen Beulphänomenen nicht gegeben ist. Alternativ hierzu definiert Spohr [139] mit der effektiven Bezugsgröße

$$
B^{*}=\alpha_{l} \frac{E t^{3}}{12\left(1-v^{2}\right)}
$$

die bezogene Störenergie

$$
\pi_{c r}=\frac{\Pi_{c r, M}}{B^{*}} .
$$

Mit dem Parameter $\alpha_{l}$ kann bei Kreiszylinderschalen unter Axiallast, Radialdruck oder Torsion der Einfluss des Längen-Radius-Verhältnisses $l / r$ auf die Imperfektionsempfindlichkeit erfasst werden. Für andere Schalentragwerke ist der Parameter $\alpha_{l}$ nicht definiert. Daher wird der Parameter im Rahmen dieser Arbeit zu eins gesetzt. Die Bedeutung des Elastizitätsmoduls und der Wanddicke für die Normierung der Störenergie ist in [139], die der Querdehnzahl in Abschnitt 4.3.4 aufgezeigt.

\subsubsection{Statische und kinetische Grenzlasten}

Die numerische Identifikation der statischen Grenzlast kann für ausgewählte Beulfälle anhand des Vergleiches der Belastung, die verschiedenen Grundzuständen zugehörig ist, mit den Grenzlasten gemäß Experimenten oder Regelwerken erfolgen. Der Vergleich ermöglicht die Festlegung eines Wertes der bezogenen Störenergie, mit dem realistische Grenzlasten beschreibbar sind. Für in Radial- und Umfangsrichtung unverschieblich, gelenkig gelagerte Kreiszylinderschalen unter Axiallast gibt Knoke [87] den Wert mit $\pi_{c r}=2,7 \%$ an. Dieser Wert wird im Weiteren als Referenzwert der bezogenen Störenergie bezeichnet. Mit dem Referenzwert können die in den Regelwerken angegebenen Grenzlasten verschiedener Beulfälle, aber auch die Grenzlasten von in den Regelwerken nicht diskutierten Beulfällen bewertet werden. 
Kinetische Grenzlasten von Schalentragwerken können bei elastischem Materialverhalten mit dem Stabilitätsgrad nach Dinkler [32]

$$
\eta=\frac{\Pi_{c r, N}-W_{e x t}-W_{0}}{\Pi_{c r, N}} \begin{cases}>0 & : \text { stabil } \\ =0 & : \text { indifferent } \\ <0 & : \text { instabil }\end{cases}
$$

ermittelt werden. Der Stabilitätsgrad folgt aus der Bewertung der Energiebilanz (2.7) für die Dauer $t_{p}$ einer kurzzeitigen kinetischen Störlast und ist ein lastniveauspezifisches Maß für die Stabilität von Schalentragwerken. Zur Berechnung der von der kinetischen Störlast in ein System eingeprägten Energie

$$
W_{e x t}=\int_{t_{0}=0}^{t_{p}} \dot{\mathbf{z}}^{T} \mathbf{p}_{p}(t) d t=\int_{t_{0}=0}^{t_{p}} \dot{\Pi}_{s t r}+\dot{T} d t
$$

bzw. des Stabilitätsgrades ist die semidiskrete Bewegungsgleichung (2.30) nur für die Dauer der Störlast zu integrieren, sofern nach deren Einwirken eine autonome Bewegung des Systems vorliegt. Infolge der Anfangsbedingungen $\mathbf{z}_{0}, \dot{\mathbf{z}}_{0}$ einer am Grundzustand $F$ initiierten Bewegung wird mit $\Delta \mathbf{z}=\mathbf{z}_{0}-\mathbf{z}_{F}$ und $\Delta \dot{\mathbf{z}}=\dot{\mathbf{z}}_{0}$ die Energie

$$
W_{0}=\frac{1}{2} \Delta \mathbf{z}^{T} \mathbf{A}_{T}\left(\mathbf{z}_{F}\right) \Delta \mathbf{z}+\frac{1}{6} \Delta \mathbf{z}^{T} \mathbf{A}_{N L}(\Delta \mathbf{z}) \Delta \mathbf{z}+\frac{1}{2} \Delta \dot{\mathbf{z}}^{T} \mathbf{M} \Delta \dot{\mathbf{z}}
$$

in das System eingeprägt. Mit dem Stabilitätsgrad ist im Vergleich zu konventionellen Stabilitätskriterien eine effiziente Bewertung der Stabilität eines Grundzustandes möglich. Dies wird deutlich, wenn die Stabilität mehrerer Grundzustände für unterschiedliche kinetische Störlasten zu bewerten ist. Zur Verifikation des Stabilitätsgrades ist, wie in Abschnitt 8.1 aufgezeigt, insbesondere Störlast (4.7) geeignet.

\subsubsection{Einflussfaktoren}

Die Imperfektionsempfindlichkeit und die Grenzlasten von Schalentragwerken sind von verschiedenen Einflussfaktoren bzw. Parametern abhängig. Zu den wichtigsten geometrischen Parametern zählen u.a. die Schlankheit $r / t$, die bei Kugelschalen den Regelwerken $[28,31]$ folgend mit $R / t$ bezeichnet wird, das Längen-Radius-Verhältnis $l / r$ und die Gauß'sche Krümmung. Lastseitige Parameter sind die Art und das Niveau der Grundbelastung und der Störlast. Für Schalentragwerke aus Stahl besitzen der Elastizitätsmodul und die Fließspannung signifikanten Einfluss auf das Tragverhalten. Dieses wird auch von der Art der Randbedingungen, z. B. kontinuierlich oder diskret, beeinflusst. Die Bedeutung einzelner Einflussfaktoren für das Tragverhalten von Schalentragwerken wird in den nachfolgenden Kapiteln analysiert. In diesem 
Abschnitt wird der Einfluss der Diskretisierung der Lagrange-Funktion (2.9) sowie der Einfluss der Querdehnzahl und der in der Störenergie enthaltenen Membranenergie auf die Bewertung der Imperfektionsempfindlichkeit und der Grenzlasten erörtert.

Diskretisierung. Bei der numerischen Bewertung der Imperfektionsempfindlichkeit ist der Einfluss der Diskretisierung der Lagrange-Funktion (2.9) zu beachten. Da die Störenergie im Wesentlichen Biegeenergie repräsentiert, sind zur Erfassung des Einflusses der räumlichen Diskretisierung auch Kriterien geeignet, die nur die Biegetragwirkung berücksichtigen. Ein entsprechendes Kriterium stellt Knoke [87] mit dem dimensionslosen Elementierungsgrad $k_{\text {Ele }}$ vor. Mit diesem folgt die maximale Elementlänge $l_{e}$ innerhalb der halben Periodenlänge einer Biegestörung zu

$$
l_{e}=\frac{L}{2 k_{E l e}} .
$$

Die Periodenlänge $L$ ist mit Hilfe der linearen Schalenbiegetheorie berechenbar [107]. Der Elementierungsgrad ist in Abhängigkeit von dem Elementtyp und der Güte der Approximation der Störenergie festzulegen. Für gemischte Elemente mit bilinearen Ansätzen wird $k_{E l e}=6$ empfohlen [87]. Für gemischte Elemente mit biquadratischen Ansätzen ist ein adäquater Elementierungsgrad noch zu ermitteln. Hierzu wird für eine Kugelschale unter Radialdruck die Konvergenz der bezogenen Störenergie, der Beullast und der mit dem Referenzwert der bezogenen Störenergie definierten Grenzlast in Abhängigkeit vom Elementierungsgrad analysiert, vgl. Bild 4.5.
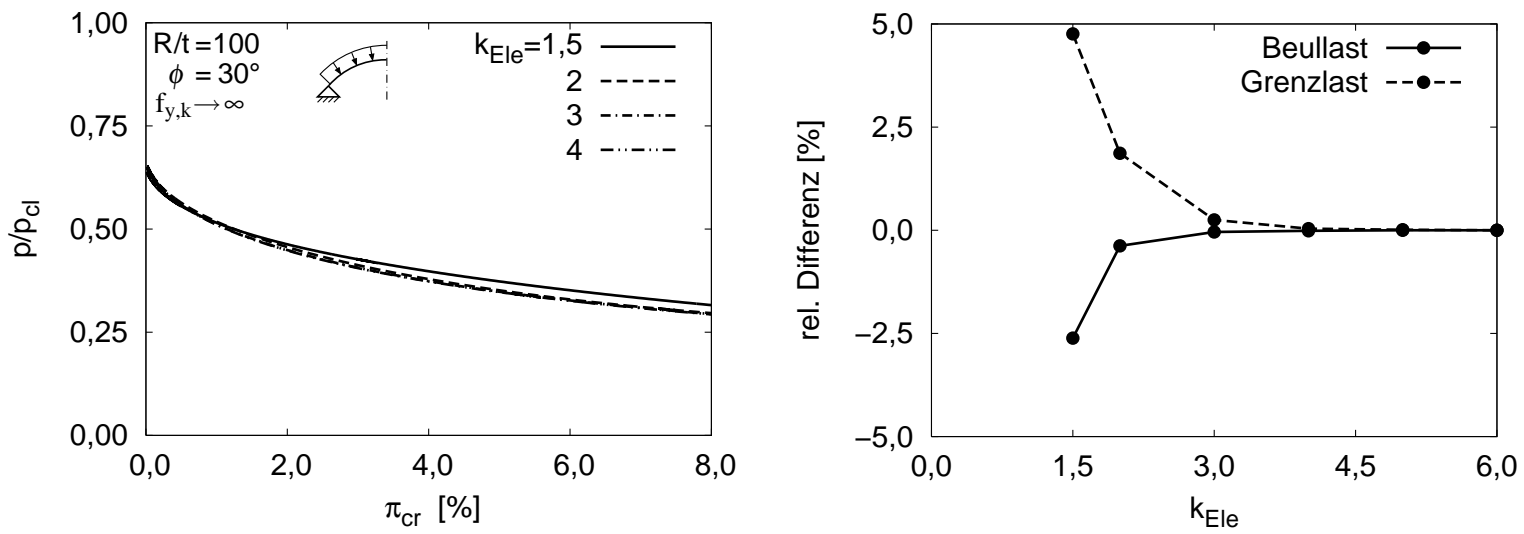

Bild 4.5: Bezogene Störenergie, Beul- und Grenzlast vs. Diskretisierung

Der Verlauf der bezogenen Störenergie zeigt für Elementierungsgrade $k_{E l e}>2$ nur geringfügige Änderungen. Im Bereich des Referenzwertes der bezogenen Störenergie nähern sich die Verläufe der bezogenen Störenergie von oben der exakten Lösung. Dies verdeutlicht der Verlauf der Grenzlast. Die relative Differenz zwischen der Grenzlast bei $k_{\text {Ele }}=3$ und $k_{\text {Ele }}=6$ beträgt $0,25 \%$, die entsprechende Differenz der Beullast $0,04 \%$. Die Differenzen werden nur geringfügig von der Schlankheit beeinflusst und resultieren wie die unterschiedliche Abhängigkeit der Beul- und der Grenzlast von der Diskretisierung aus der Approximation der geometrischen Nichtlinearitäten. Un- 
ter Berücksichtigung von Berechnungsaufwand und Approximationsgüte wird den nachfolgenden Analysen bei Verwendung gemischter Elemente mit biquadratischen Ansätzen, sofern nicht anders angegeben, ein Elementierungsgrad $k_{E l e}=3$ zugrunde gelegt. Der Einfluss der räumlichen und zeitlichen Diskretisierung der LagrangeFunktion auf die Genauigkeit des Stabilitätsgrades wird in Abschnitt 8.1 bewertet.

Der Elementierungsgrad erlaubt die räumliche Diskretisierung der Lagrange-Funktion in Abhängigkeit von der räumlichen Verteilung der Störlast bzw. der entsprechenden Änderung der Zustandsgrößen. Die Diskretisierung kann eine Verfeinerung im Bereich der Störlast aufweisen und wird i.d.R. für alle Lastniveaus beibehalten. Ist die räumliche Verteilung der Störlast signifikant vom Lastniveau abhängig, so ist die Diskretisierung ggf. zu optimieren, um eine Bewertung der Imperfektionsempfindlichkeit mit annähernd gleicher Genauigkeit zu gewährleisten. Entsprechendes gilt, wenn der Einfluss unterschiedlicher Störlasten auf die Stabilität eines Grundzustandes zu bewerten ist. Bei Schalentragwerken mit beliebiger Geometrie oder Öffnungen sowie bei Verwendung geometrisch verzerrter Elemente ist der Elementierungsgrad nur bedingt zur Bewertung des Einflusses der räumlichen Diskretisierung geeignet. In diesen Fällen kann eine adaptive Verfeinerung der Diskretisierung in Abhängigkeit von der Konvergenz der Störenergie vorteilhaft sein.

Querdehnzahl. Der Einfluss der Querdehnzahl auf das Tragverhalten und die Normierung der Störenergie wird exemplarisch für eine Kreiszylinderschale unter Axiallast untersucht. Die Kreiszylinderschale ist an beiden Rändern in Radial- und Umfangsrichtung unverschieblich gelagert. Bild 4.6 links stellt die Abhängigkeit der Beullast und des Verlaufes der Störenergie $\Pi_{c r, M}$ von der Querdehnzahl heraus.
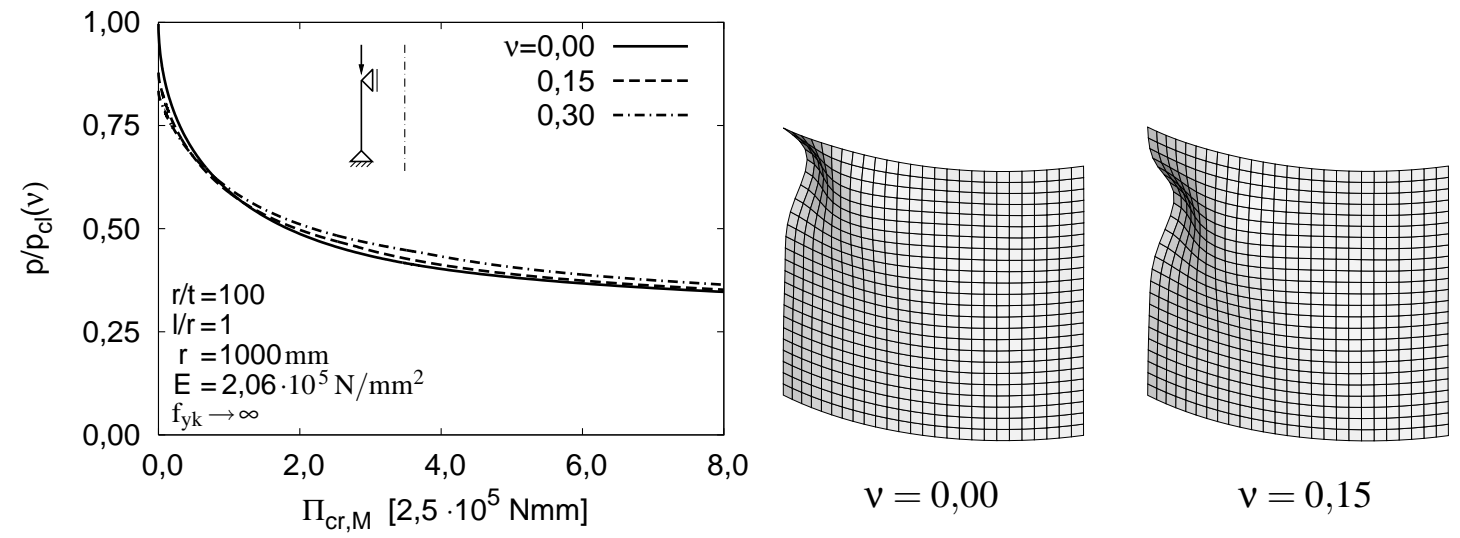

Bild 4.6: Verlauf der Störenergie und Verformungen infolge Störlast

Mit zunehmender Querdehnzahl vergrößern sich die Randstörungen infolge Grundbelastung, womit eine Verringerung der Beullast einhergeht. Der Verlauf der Störenergie ist für unterschiedliche Querdehnzahlen zwar ähnlich, die Störenergien stehen aber nicht in einem bestimmten, vom Lastniveau unabhängigen Verhältnis zueinander. Dies verdeutlicht eine Betrachtung der Störenergie oberhalb und unterhalb 
des Lastniveaus $p / p_{c l}=0,62$. Oberhalb dieses Lastniveaus nimmt die Störenergie für einen definierten Grundzustand mit zunehmender Querdehnzahl ab, unterhalb dagegen zu. Für das Lastniveau $p / p_{c l}=0,62$ ist die Störenergie nur geringfügig von der Querdehnzahl abhängig. Für Lastniveaus im Bereich des Nachbeulminimums ist die Imperfektionsempfindlichkeit eines Grundzustandes bei kleinen Querdehnzahlen nahezu identisch. Der im Vergleich zum Elastizitätsmodul und zur Wanddicke unterschiedliche Einfluss der Querdehnzahl auf das Tragverhalten und die Normierung der Störenergie ist mit den Komponenten des Elastizitätstensors zu begründen.

Neben der Störenergie ist auch die räumliche Verteilung der inkrementellen Zustandsgrößen $\Delta \mathbf{z}_{c r, M}$ von der Querdehnzahl abhängig. Entsprechende Verformungen sind in Bild 4.6 rechts 15-fach überhöht dargestellt. Für Querdehnzahlen größer null sind die Verformungen in geringem Abstand vom oberen Rand lokalisiert. Dahingegen sind bei einer Querdehnzahl identisch null die Verformungen unmittelbar am oberen Rand konzentriert, da Randstörungen infolge Grundbelastung nicht auftreten und somit der Bereich am oberen Rand am imperfektionsempfindlichsten ist. Allgemein ist der Einfluss der Querdehnzahl auf die Randstörungen infolge Grundbelastung vom Beulfall abhängig. Zusammenfassend ist festzuhalten, dass Normierung (4.20) für Beulfälle mit unterschiedlicher Querdehnzahl nur bedingt geeignet ist.

Membranenergieanteil. Im Rahmen der gewählten gemischten Formulierung, der gemäß Abschnitt 2.2 die Kirchhoff-Love-Hypothese zugrunde liegt, besteht die Störenergie bei elastischem Materialverhalten aus Membran- und Biegeenergie. Mit Normierung (4.20) ist aber die gesamte Störenergie auf die effektive Bezugsgröße zu beziehen. Diesbezüglich wird nachfolgend die Normierung für eine Kugelschale unter Radialdruck bewertet. Hierzu wird die bezogene Störenergie entsprechend Gl. (4.15b) in die Anteile $\pi_{c r, 1}, \pi_{c r, 2}, \pi_{c r, 3}$ sowie in die Membranenergie $\pi_{c r, M e m}$ und Biegeenergie $\pi_{c r, B e n}$ getrennt. Bild 4.7 zeigt die Abhängigkeit der Anteile vom Lastniveau auf.
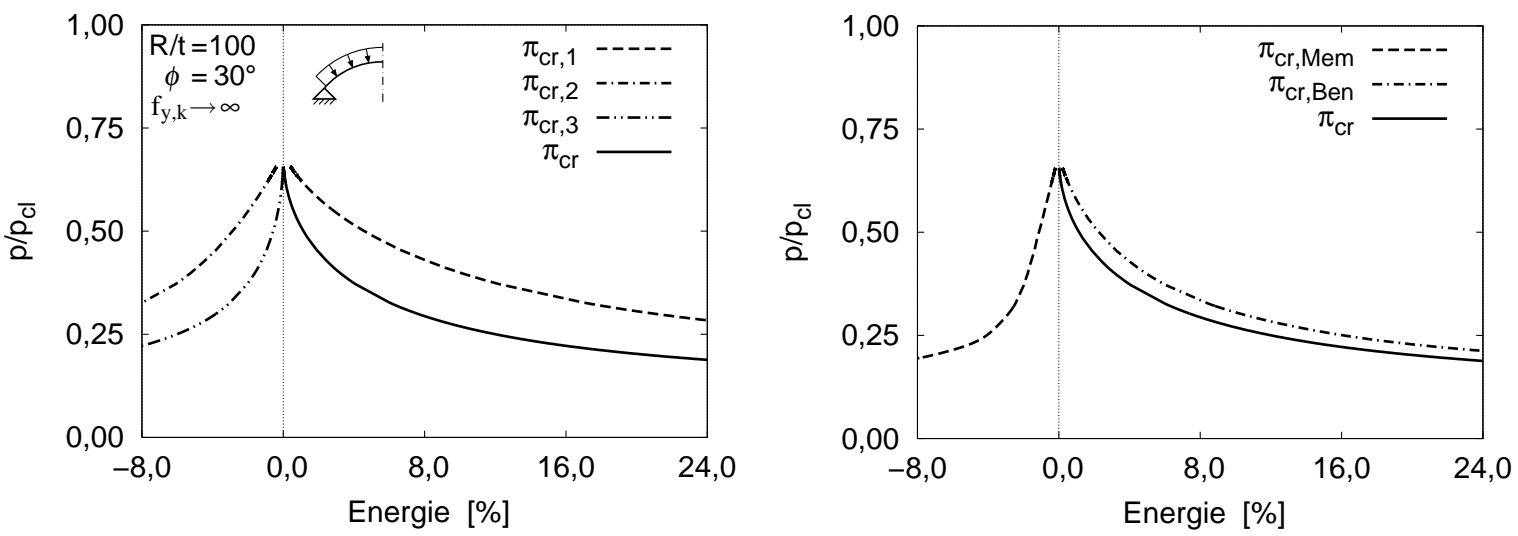

Bild 4.7: Anteile der bezogenen Störenergie vs. Lastniveau

Der Anteil $\pi_{c r, 1}$ besteht aus positiver Membran- und Biegeenergie und ist dem linken Diagramm zufolge für alle Lastniveaus der dominierende Anteil der bezogenen Stör- 
energie. Dahingegen repräsentieren die Anteile $\pi_{c r, 2}$ und $\pi_{c r, 3}$ negative Membranenergien, deren Summe, wie das rechte Diagramm verdeutlicht, gegenüber der Membranenergie des Anteiles $\pi_{c r, 1}$ überwiegt. Das Verhältnis von Membran- zu Biegeenergie ist vom Lastniveau abhängig. Unabhängig vom Lastniveau stellt allerdings Biegeenergie den wesentlichen Anteil der bezogenen Störenergie dar. Somit ist diese primär in den Randbereichen des Schalenkontinuums konzentriert und ermöglicht Gl. (4.20) eine phänomenologisch begründete und, wie in [139, 87] gezeigt, besonders geeignete Normierung der Störenergie. Die Normierung wird in Abschnitt 5.4 zur Anwendung auf Schalentragwerke aus Faserverbundwerkstoff weiterentwickelt.

\subsection{Berücksichtigung weiterer nicht anfänglicher Störungen}

Zur Analyse des Einflusses nicht anfänglicher Störungen auf das Tragverhalten ist das inkrementelle elastische Potential in Abhängigkeit von der Änderung der Parameter des inneren elastischen und des äußeren Potentials zu formulieren. Die Berücksichtigung einer Änderung sämtlicher Parameter des inneren elastischen Potentials erfordert großen Berechnungsaufwand. Daher werden im Rahmen dieser Arbeit neben der Änderung der Belastung exemplarisch nur Änderungen der Wanddicke $t$ und des Bettungsmoduls $K_{B}$ berücksichtigt. Diese beiden Parameter sind ursprünglich nur in der Matrix $\mathbf{A}_{L}$ enthalten, wobei die Wanddicke mit ihrem reziproken Wert eingeht. Folglich sind für die Terme des elastischen Potentials, welche die Wanddicke enthalten, sämtliche höheren Variationen ungleich null. Der Einfluss einer Änderung der Wanddicke auf das Tragverhalten ist daher nur näherungsweise zu erfassen. Unter Berücksichtigung einer Änderung beider Parameter folgt mit den ersten vier Variationen des elastischen Potentials das inkrementelle elastische Potential zu

$$
\begin{aligned}
\Delta \Pi & =\frac{1}{2} \Delta \mathbf{z}^{T} \mathbf{A}_{T}\left(\mathbf{z}_{F}\right) \Delta \mathbf{z}+\frac{1}{6} \Delta \mathbf{z}^{T} \mathbf{A}_{N L}(\Delta \mathbf{z}) \Delta \mathbf{z}-\Delta \mathbf{z}^{T} \Delta \mathbf{p} \\
& +\frac{1}{2} \Delta \mathbf{z}^{T} \mathbf{A}_{\Delta L_{0}} \Delta \mathbf{z}+\Delta \mathbf{z}^{T} \mathbf{A}_{\Delta L_{1}} \mathbf{z}_{F}+\frac{1}{2} \mathbf{z}_{F}^{T} \mathbf{A}_{\Delta L_{2}} \mathbf{z}_{F},
\end{aligned}
$$

mit den Matrizen

$$
\mathbf{A}_{\Delta L_{i(i=0,1)}}=\left[\begin{array}{ccc}
-f_{D_{i}} \mathbf{F}_{D} & \mathbf{0} & \mathbf{0} \\
\mathbf{0} & -f_{B_{i}} \mathbf{F}_{B} & \mathbf{0} \\
\mathbf{0} & \mathbf{0} & \Delta \mathbf{K}_{\mathbf{B}}
\end{array}\right], \mathbf{A}_{\Delta L_{2}}=\left[\begin{array}{ccc}
-f_{D_{2}} \mathbf{F}_{D} & \mathbf{0} & \mathbf{0} \\
\mathbf{0} & -f_{B_{2}} \mathbf{F}_{B} & \mathbf{0} \\
\mathbf{0} & \mathbf{0} & \mathbf{0}
\end{array}\right]
$$

und den Parametern

$$
\begin{aligned}
& f_{D_{0}}=-\frac{\Delta t}{t}+\frac{\Delta t^{2}}{t^{2}}, f_{D_{1}}=-\left(1+f_{D_{0}}\right) \frac{\Delta t}{t}, f_{D_{2}}=\left(1+f_{D_{0}}\right) \frac{\Delta t^{2}}{t^{2}}, \\
& f_{B_{0}}=-3 \frac{\Delta t}{t}+6 \frac{\Delta t^{2}}{t^{2}}, f_{B_{1}}=-3 \frac{\Delta t}{t}+6 \frac{\Delta t^{2}}{t^{2}}-10 \frac{\Delta t^{3}}{t^{3}}
\end{aligned}
$$




$$
f_{B_{2}}=6 \frac{\Delta t^{2}}{t^{2}}-10 \frac{\Delta t^{3}}{t^{3}}+15 \frac{\Delta t^{4}}{t^{4}} .
$$

Bei Berücksichtigung höherer Variationen ändern sich nur die Parameter (4.27). Potential (4.25) beschreibt Änderungen der Energiefläche im Bereich eines Grundzustandes und besitzt im Vergleich zum Potential (2.54) drei zusätzliche Anteile. Der erste Anteil beeinflusst die Krümmung und der zweite die Neigung der Energiefläche. Der dritte Anteil bedingt bei einer im Inkrement konstanten Änderung der Wanddicke eine Änderung des Energieniveaus des Grundzustandes. Der Einfluss der zusätzlichen Anteile auf die Topologie der Energiefläche ist von der räumlichen Verteilung der nicht anfänglichen Störungen sowie von den Zustandsgrößen im Grundzustand und deren inkrementeller Änderung abhängig. Sofern der zweite Anteil identisch null ist, sind kritische Zustände in Analogie zu den Eigenwertproblemen (4.8) mit

$$
\left[\left(\mathbf{A}_{T}\left(\mathbf{z}_{F}\right)+\mathbf{A}_{\Delta L_{0}}\right)+\lambda \xi \mathbf{A}_{N L}(\boldsymbol{\phi})\right] \boldsymbol{\phi}=\mathbf{0}, \text { mit } \xi=\frac{1}{2} \text { bzw. 1,0 }
$$

identifizierbar. Ansonsten müssen kritische Zustände $N$ mit dem aus der ersten Variation des Potentials (4.25) resultierenden inhomogenen Gleichungssystem ermittelt werden. Aus der ersten Variation folgt ferner, dass Störlast (4.7) um zwei Anteile zu ergänzen ist. Die Störenergie (4.15) ist nur um die ersten beiden zusätzlichen Anteile zu erweitern, da der dritte Anteil die zur Identifikation kritischer Zustände relevanten Eigenschaften der Energiefläche nicht beeinflusst und nicht aus dem Wirken der Störlast resultiert. Die Identifikation kritischer Zustände im Fall nicht anfänglicher Störungen ist Ausgangspunkt des Gedankens der in Abschnitt 8.3 vorgestellten Möglichkeit zur lastniveauspezifischen Modifikation der Imperfektionsempfindlichkeit.

\subsection{Bewertung des Einflusses anfänglicher Störungen}

Anfängliche Störungen bzw. Imperfektionen können deutliche Änderungen des Tragverhaltens bewirken. Dies ist u.a. am Lastniveau des ersten singulären Punktes im primären Last-Verformungs-Pfad zu erkennen. Die Bewertung des Einflusses anfänglicher Störungen auf das Tragverhalten gelingt auch mittels energetischer Betrachtungen. Eine Möglichkeit ist die Analyse der störungsbedingten Änderung der zum ersten singulären Zustand eines Systems korrespondierenden Grundenergie. Eine weitere Möglichkeit ist die Bewertung der störungsbedingten Änderung der Störenergie,

$$
\Delta \Pi_{c r}^{\operatorname{Imp}}\left(\mathbf{z}_{F}, \mathbf{z}_{\text {Imp }}\right)=\Pi_{c r}\left(\Delta \mathbf{z}_{c r}, 0\right)-\Pi_{c r}\left(\Delta \mathbf{z}_{c r, \text { Imp }}, \mathbf{z}_{\text {Imp }}\right) .
$$

Hierzu sind die Eigenwertprobleme (4.8) zusätzlich unter Berücksichtigung der anfänglichen Störungen $\mathbf{z}_{\text {Imp }}$, die sowohl Störungen der Geometrie oder als auch Störungen des Spannungszustandes umfassen können, zu lösen [32]. 
Die Bestimmung einer ungünstigen anfänglichen Störung ist möglich, wenn das Störungsfeld als zusätzliche unbekannte Größe in das mechanische Modell eingeht [34, 30]. Die zusätzlichen, zur Berechnung der Freiwerte des diskretisierten Störungsfeldes erforderlichen Gleichungen folgen aus der ersten Variation des elastischen Potentials nach dem Störungsfeld. Weitere Gleichungen resultieren aus der ersten und zweiten Variation des Potentials nach den Zustandsgrößen, wobei letztere die Bedingung zur Identifikation eines singulären Punktes darstellt. Die Gleichungen bilden zusammen mit etwaigen Normierungsbedingungen ein erweitertes Gleichungssystem. Wird das Gleichungssystem für ein definiertes Lastniveau gelöst, so ist die Identifikation einer hinsichtlich Form, Amplitude und Position ungünstigsten anfänglichen Störung denkbar. Interessant ist der Fall, in dem das so definierte Lastniveau die Grenzlast gemäß Referenzwert der bezogenen Störenergie repräsentiert.

\subsection{Weggrößenformulierung}

Den bisherigen Ausführungen zum Störenergie-Konzept liegt eine gemischte Formulierung zugrunde. Nachfolgend wird die Anwendung des Störenergie-Konzeptes im Rahmen der in Abschnitt 2.2 vorgestellten Weggrößenformulierung erörtert.

\subsubsection{Identifikation kritischer Zustände und Störenergie}

Im Unterschied zur gemischten Formulierung ist in der Weggrößenformulierung auch die vierte Variation des elastischen Potentials nach den Zustandsgrößen ungleich null. Daher besteht das inkrementelle innere Potential aus drei Anteilen. In Analogie zu den Eigenwertproblemen (4.8) sind im Rahmen der Weggrößenformulierung ein Eigenwertproblem zur Identifikation kritischer Zustände $N$,

$$
\left[\mathbf{K}_{0}\left(\mathbf{v}_{F}, \mathbf{v}_{F}\right)+\frac{1}{2} \lambda_{N} \mathbf{K}_{1}\left(\mathbf{v}_{F}, \boldsymbol{\phi}\right)+\frac{1}{6} \lambda_{N}^{2} \mathbf{K}_{2}(\boldsymbol{\phi}, \boldsymbol{\phi})\right] \boldsymbol{\phi}=\mathbf{0},
$$

und formal ein Eigenwertproblem zur Identifikation kritischer Zustände $M$,

$$
\left[\mathbf{K}_{0}\left(\mathbf{v}_{F}, \mathbf{v}_{F}\right)+\lambda_{M} \mathbf{K}_{1}\left(\mathbf{v}_{F}, \boldsymbol{\phi}\right)+\frac{1}{2} \lambda_{M}^{2} \mathbf{K}_{2}(\boldsymbol{\phi}, \boldsymbol{\phi})\right] \boldsymbol{\phi}=\mathbf{0},
$$

herleitbar. Beide Eigenwertprobleme weisen eine quadratische Abhängigkeit vom Eigenwert und eine kubische Abhängigkeit vom Eigenvektor auf. Die Eigenwertprobleme können mit der Identität

$$
\mathbf{K}_{0} \lambda \boldsymbol{\phi}-\lambda \mathbf{K}_{0} \boldsymbol{\phi}=\mathbf{0}
$$


und den Koeffizienten $\xi_{1}$ und $\xi_{2}$, welche die von der Art des kritischen Zustandes abhängigen Vorfaktoren der Matrizen $\mathbf{K}_{1}$ und $\mathbf{K}_{2}$ repräsentieren, in die Form [87]

$$
\left\{\left[\begin{array}{cc}
\xi_{1} \mathbf{K}_{1} & \mathbf{K}_{0} \\
\mathbf{K}_{0} & \mathbf{0}
\end{array}\right]+\lambda\left[\begin{array}{cc}
\xi_{2} \mathbf{K}_{2} & \mathbf{0} \\
\mathbf{0} & -\mathbf{K}_{0}
\end{array}\right]\right\}\left[\begin{array}{c}
\lambda \boldsymbol{\phi} \\
\boldsymbol{\phi}
\end{array}\right]=\left[\begin{array}{l}
\mathbf{0} \\
\mathbf{0}
\end{array}\right]
$$

bzw.

$$
\left[\mathbf{A}\left(\xi_{1}, \phi\right)+\lambda \mathbf{B}\left(\xi_{2}, \phi, \phi\right)\right] \boldsymbol{\phi}^{*}=\mathbf{0}
$$

transformiert werden. Die Eigenwertprobleme (4.34) besitzen die Dimension $N=2 n$, enthalten reelle symmetrische Untermatrizen und sind mit den in Abschnitt 2.5.3 vorgestellten Verfahren lösbar. Die Störenergie folgt mit $\Delta \mathbf{v}_{c r}=\lambda \phi \mathrm{zu}$

$$
\begin{aligned}
\Pi_{c r} & =\frac{1}{2} \Delta \mathbf{v}_{c r}^{T} \mathbf{K}_{0}\left(\mathbf{v}_{F}, \mathbf{v}_{F}\right) \Delta \mathbf{v}_{c r}+\frac{1}{6} \Delta \mathbf{v}_{c r}^{T} \mathbf{K}_{1}\left(\mathbf{v}_{F}, \Delta \mathbf{v}_{c r}\right) \Delta \mathbf{v}_{c r} \\
& +\frac{1}{24} \Delta \mathbf{v}_{c r}^{T} \mathbf{K}_{2}\left(\Delta \mathbf{v}_{c r}, \Delta \mathbf{v}_{c r}\right) \Delta \mathbf{v}_{c r} .
\end{aligned}
$$

Im Unterschied zur gemischten Formulierung besteht die Störenergie in der Weggrößenformulierung aus drei und die Grundenergie aus vier Anteilen. Sofern ein kritischer Zustand $M$ mit Eigenwertproblem (4.31) ermittelbar ist, umfasst die zugehörige Störlast nur Kraftgrößen. Eine alternative Formulierung der Eigenwertprobleme (4.34) ist mittels Zeilentausches und Abspeicherung des Terms $\xi_{1} \mathbf{K}_{1}$ in der Matrix $\mathbf{B}$ herzuleiten. In diesem Fall ist die Matrix $\mathbf{B}$ zwar unsymmetrisch, die Matrix $\mathbf{A}$ jedoch unabhängig vom Eigenvektor, symmetrisch und von geringerer Bandbreite.

\subsubsection{Verifikationsbeispiel 1 - Stabtragwerk}

Die Imperfektionsempfindlichkeit des in Bild 4.8 dargestellten Stabtragwerkes wird mit dem Störenergie-Konzept im Rahmen der Weggrößen- und der gemischten Formulierung bewertet. Dabei ist linear elastisches Materialverhalten vorausgesetzt und ein Knicken des Systems ausgeschlossen. Die Diskretisierung der Verschiebung in vertikaler Richtung erfolgt mit einem linearen Ansatz. Somit kann die Kinematik

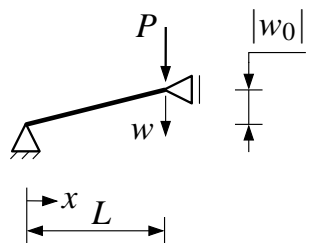

Bild 4.8: Stabtragwerk des Systems unter Berücksichtigung der Randbedingungen, des Knotenfreiwertes $w$ und dessen anfänglicher Störung $w_{0}$ sowie mit der Länge $L=1 \mathrm{~m} \mathrm{zu}$

$$
\varepsilon=\frac{1}{2} w^{2}+w w_{0}
$$

beschrieben werden. Zusätzlich wird in der gemischten Formulierung die Normalkraft mit einem konstanten Ansatz abgebildet. Es ist zu erwarten, dass beide FE-Formulierungen auf identische Verläufe der Störenergie führen. 
Weggrößenformulierung. Einziger Freiwert des Systems ist die Verschiebung $w$ des Lastangriffspunktes. Das inkrementelle elastische Potential des Systems ist mit

$$
\Delta \Pi=\frac{1}{2} \Delta \mathbf{v}^{T} \mathbf{K}_{0} \Delta \mathbf{v}+\frac{1}{6} \Delta \mathbf{v}^{T} \mathbf{K}_{1} \Delta \mathbf{v}+\frac{1}{24} \Delta \mathbf{v}^{T} \mathbf{K}_{2} \Delta \mathbf{v}-\Delta \mathbf{v}^{T} \Delta \mathbf{p}
$$

gegeben, wobei

$$
\begin{aligned}
& \mathbf{K}_{0}=\left[E A\left(w_{0}^{2}+3,0 w_{0} w+1,5 w^{2}\right)\right], \mathbf{K}_{1}=\left[3,0 E A\left(w+w_{0}\right) \Delta w\right], \\
& \mathbf{K}_{2}=\left[3,0 E A \Delta w^{2}\right], \Delta \mathbf{v}=[\Delta w], \Delta \mathbf{p}=[\Delta P] .
\end{aligned}
$$

Die ersten zwei Variationen des inkrementellen Potentials,

$$
\begin{aligned}
\delta \Delta \Pi=\delta \Delta \mathbf{v}^{T}\left(\mathbf{K}_{0} \Delta \mathbf{v}+\frac{1}{2} \mathbf{K}_{1} \Delta \mathbf{v}+\frac{1}{6} \mathbf{K}_{2} \Delta \mathbf{v}-\Delta P\right)=0, \\
\delta^{2} \Delta \Pi=\delta \Delta \mathbf{v}^{T}\left(\mathbf{K}_{0}+1,0 \mathbf{K}_{1}+\frac{1}{2} \mathbf{K}_{2}\right) \delta \Delta \mathbf{v}=0,
\end{aligned}
$$

führen mit $\Delta w=\lambda \phi$ und entsprechend den Ausführungen in Abschnitt 4.2.1 auf die Eigenwertprobleme zur Identifikation kritischer Zustände $N$ und $M$,

$$
\left[\mathbf{K}_{0}(w, w)+\lambda \xi_{1} \mathbf{K}_{1}(w, \phi)+\lambda^{2} \xi_{2} \mathbf{K}_{2}(\phi, \phi)\right] \phi=\mathbf{0} \text {. }
$$

Gemischte Formulierung. Freiwerte des Systems sind die Normalkraft $N$ und die Verschiebung $w$ des Lastangriffspunktes. Das inkrementelle elastische Potential des Systems ist gegeben mit

$$
\Delta \Pi=\left[\begin{array}{l}
\Delta N \\
\Delta w
\end{array}\right]^{T}\left[\left\{\frac{1}{2}\left[\begin{array}{cc}
-1 / E A & w+w_{0} \\
w+w_{0} & N
\end{array}\right]+\frac{1}{6}\left[\begin{array}{cc}
0 & \Delta w \\
\Delta w & \Delta N
\end{array}\right]\right\}\left[\begin{array}{c}
\Delta N \\
\Delta w
\end{array}\right]-\left[\begin{array}{c}
0 \\
\Delta P
\end{array}\right]\right] .
$$

Die erste Variation des inkrementellen Potentials,

$$
\delta \Delta \Pi=\left[\begin{array}{l}
\delta \Delta N \\
\delta \Delta w
\end{array}\right]^{T}\left[\left\{\left[\begin{array}{cc}
-1 / E A & w+w_{0} \\
w+w_{0} & N
\end{array}\right]+\frac{1}{2}\left[\begin{array}{cc}
0 & \Delta w \\
\Delta w & \Delta N
\end{array}\right]\right\}\left[\begin{array}{c}
\Delta N \\
\Delta w
\end{array}\right]-\left[\begin{array}{c}
0 \\
\Delta P
\end{array}\right]\right]=0,
$$

führt mit

$$
\begin{aligned}
N & =\frac{1}{2} E A w^{2}+E A w w_{0}, \\
\Delta N & =N_{2}-N_{1}=\frac{1}{2} E A \Delta w \Delta w+E A\left(w+w_{0}\right) \Delta w, \\
\delta \Delta N & =E A \Delta w \delta \Delta w+E A\left(w+w_{0}\right) \delta \Delta w
\end{aligned}
$$

auf die in G1. (4.38) angegebene erste Variation des inkrementellen Potentials in Weggrößenformulierung. Die zweite Variation des inkrementellen Potentials (4.41),

$$
\delta^{2} \Delta \Pi=\left[\begin{array}{l}
\delta \Delta N \\
\delta \Delta w
\end{array}\right]^{T}\left\{\left[\begin{array}{cc}
-1 / E A & w+w_{0} \\
w+w_{0} & N
\end{array}\right]+1,0\left[\begin{array}{cc}
0 & \Delta w \\
\Delta w & \Delta N
\end{array}\right]\right\}\left[\begin{array}{l}
\delta \Delta N \\
\delta \Delta w
\end{array}\right]=0,
$$


ist mit den Gln. (4.43-4.45) in die zweite Variation des inkrementellen Potentials in Weggrößenformulierung, vgl. Gl. (4.39), transformierbar. Somit sind das inkrementelle elastische Potential und die Bedingungen einer verschwindenden ersten und zweiten Variation des Potentials jeweils für beide FE-Formulierungen einander äquivalent. Aus den Variationen (4.42) und (4.46) folgen mit $\Delta w=\lambda \phi_{\Delta w}$ sowie $\Delta N=\lambda \phi_{\Delta N}$ die Eigenwertprobleme zur Identifikation kritischer Zustände $N$ und $M$ zu

$$
\left\{\left[\begin{array}{cc}
-1 / E A & w+w_{0} \\
w+w_{0} & N
\end{array}\right]+\lambda \xi\left[\begin{array}{cc}
0 & \phi_{\Delta w} \\
\phi_{\Delta w} & \phi_{\Delta N}
\end{array}\right]\right\}\left[\begin{array}{l}
\phi_{\Delta N} \\
\phi_{\Delta w}
\end{array}\right]=\left[\begin{array}{l}
0 \\
0
\end{array}\right],
$$

wobei der Koeffizient $\xi$ von der Art des kritischen Zustandes abhängig ist.

Imperfektionsempfindlichkeit. Mit beiden FE-Formulierungen sind, wie in Bild 4.9 aufgezeigt, der primäre Last-Verformungs-Pfad, kritische Zustände $N$ und somit die Empfindlichkeit des Systems gegenüber kinetischen Störungen identisch beschrieben.
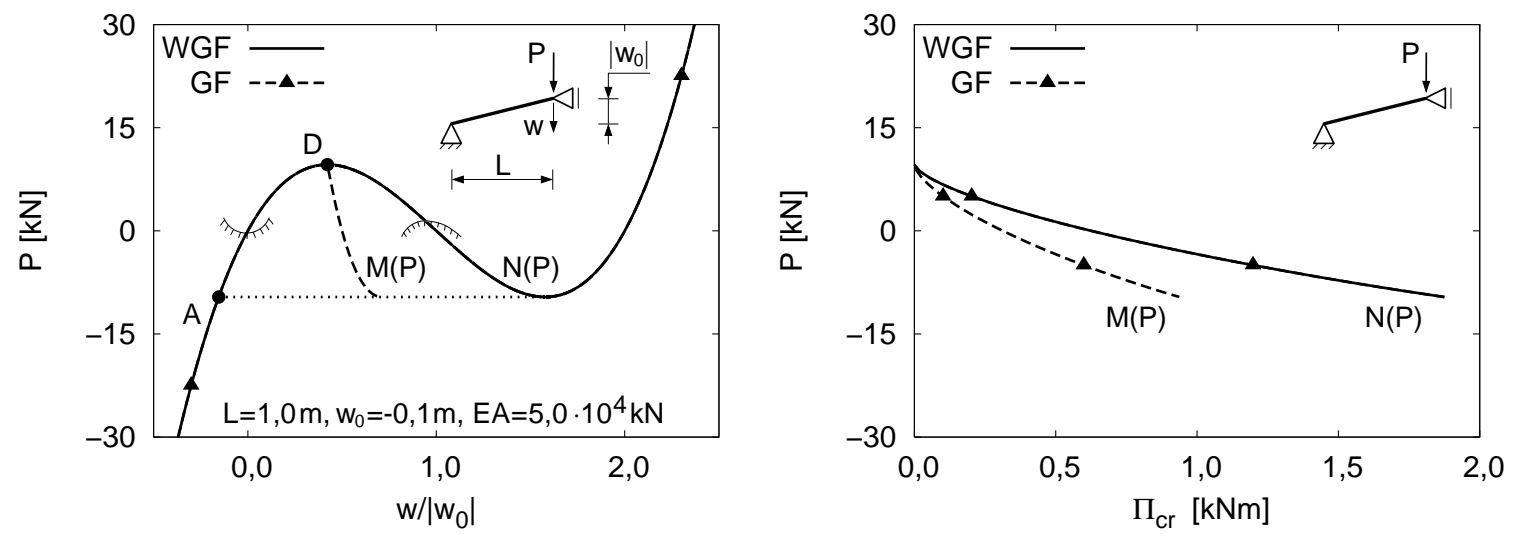

Bild 4.9: Bewertung des Tragverhaltens vs. FE-Formulierung

Die Identifikation kritischer Zustände $M$ ist in der gemischten Formulierung (GF) problemlos möglich, während in der Weggrößenformulierung (WGF) für alle Grundzustände im Bereich $A-D$ nur der Durchschlagpunkt $D$ als kritischer Zustand ermittelt wird [87]. Letzteres ist nicht korrekt, da auch andere Punkte mit verschwindender Krümmung der Energiefläche des Systems, die kritischen Zustände $M$, existieren. Daher sind die entsprechenden Eigenwertprobleme eingehender zu untersuchen.

Weggrößen- vs. gemischte Formulierung. Zur Klärung der Ursache für die misslungene Identifikation kritischer Zustände $M$ mittels Eigenwertproblem (4.40) werden aus dem Eigenwertproblem (4.47) die Normalkraft $N$ und die Komponente $\phi_{\Delta N}$ des Eigenvektors eliminiert. Die Elimination der Normalkraft erfolgt mit Gl. (4.43) und die der Komponente des Eigenvektors anhand der ersten Gleichung des Eigenwertproblems (4.47) und unter Berücksichtigung von $\xi=1,0$ und $\Delta w=\lambda \phi_{\Delta w}$ mit

$$
\phi_{\Delta N}=E A \Delta w \phi_{\Delta w}+E A\left(w+w_{0}\right) \phi_{\Delta w}
$$


Diese Gleichung unterscheidet sich nach Multiplikation mit dem Eigenwert $\lambda$ in einem Faktor von der inkrementellen kinematischen Gleichung, vgl. (4.44), und bringt eine im Gegensatz zur Weggrößenformulierung mögliche Störung der inkrementellen kinematischen Gleichung mit der Störverzerrung $\varepsilon_{p}=0,5 \lambda^{2} \phi_{\Delta w}^{2}$ zum Ausdruck,

$$
\Delta N_{p}=\lambda \phi_{\Delta N}=E A \Delta w \Delta w+E A\left(w+w_{0}\right) \Delta w=\Delta N+E A \varepsilon_{p} .
$$

Nach Elimination der Normalkraft und der Komponente des Eigenvektors aus dem Eigenwertproblem (4.47) verbleibt das Eigenwertproblem

$$
\left[\mathbf{K}_{0}+\lambda \mathbf{K}_{1}+\lambda^{2} \frac{2}{3} \mathbf{K}_{2}\right] \phi_{\Delta w}=0
$$

Dieses Eigenwertproblem differiert nur in dem Vorfaktor der Matrix $\mathbf{K}_{2}$ von dem Eigenwertproblem (4.40), ermöglicht aber die Berechnung der zu einem kritischen Zustand $M$ gehörenden Verschiebung $\Delta w_{c r}$. In einer Nachlaufrechnung ist der inkrementelle Zuwachs der Normalkraft zu ermitteln. Erfolgt die Nachlaufrechnung mit Gl. (4.44), so wird nicht der Zuwachs beim Übergang vom Grundzustand in den kritischen Zustand berechnet, sondern der Zuwachs der Normalkraft beim Übergang vom Grundzustand in einen Zustand, der zum primären Last-Verformungs-Pfad gehört. Diesen Aspekt verdeutlicht in Bild 4.10 exemplarisch der Zustand Z. Dahingegen ermöglicht Gl. (4.49) die Ermittlung der Normalkraft im kritischen Zustand $M\left(P_{A}\right)$.

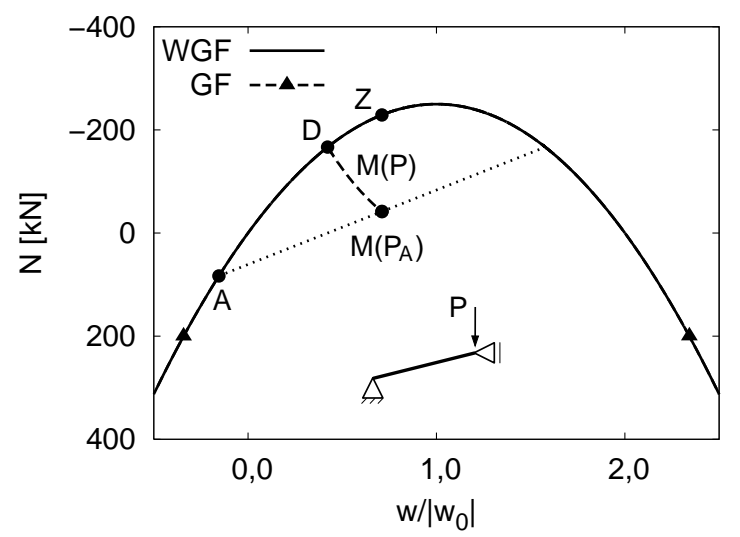

Bild 4.10: Normalkraft-Verschiebungs-Diagramm

Die Berechnung der Störenergie ist unter Berücksichtigung des inkrementellen Zuwachses der Verschiebung und der Normalkraft durchzuführen und kann daher nicht mit dem inkrementellen Potential (4.37) erfolgen. Die zwischen den Eigenwertproblemen (4.40) und (4.50) bestehende Differenz im Vorfaktor der Matrix $\mathbf{K}_{2}$ ist unabhängig von der Dimension des Systems. Entscheidend für das Misslingen der Identifikation kritischer Zustände $M$ im Rahmen der Weggrößenformulierung ist, dass Störverzerrungen definierter Größe zwar berücksichtigt, ungünstigste Störverzerrungen aber nicht berechnet werden können. Das Störenergie-Konzept ist daher im Rahmen der vorgestellten Weggrößenformulierung zwar zur Identifikation kritischer Zu- 
stände $N$, aber nicht zur Ermittlung kritischer Zustände $M$ und nicht zur Berechnung ungünstiger nicht anfänglicher Störlasten anwendbar.

\subsubsection{Verifikationsbeispiel 2 - Schalentragwerk}

Die Anwendung des Störenergie-Konzeptes im Rahmen der Weggrößenformulierung (WGF) und der gemischten Formulierung(GF) wird für das in Bild 4.11 links dargestellte und mit einer mittigen Einzellast belastete Schalentragwerk untersucht. Die Geometrie des Schalentragwerkes ist mit $r=25.400 \mathrm{~mm}, t=50,8 \mathrm{~mm}$ und $\varphi=20^{\circ}$ festgelegt. Die Materialparameter sind $E=10.350 \mathrm{~N} / \mathrm{mm}^{2}$ und $v=0$. Die Verschiebung $u_{3}$ des Lastangriffspunktes ist entgegen der Lastrichtung positiv definiert.
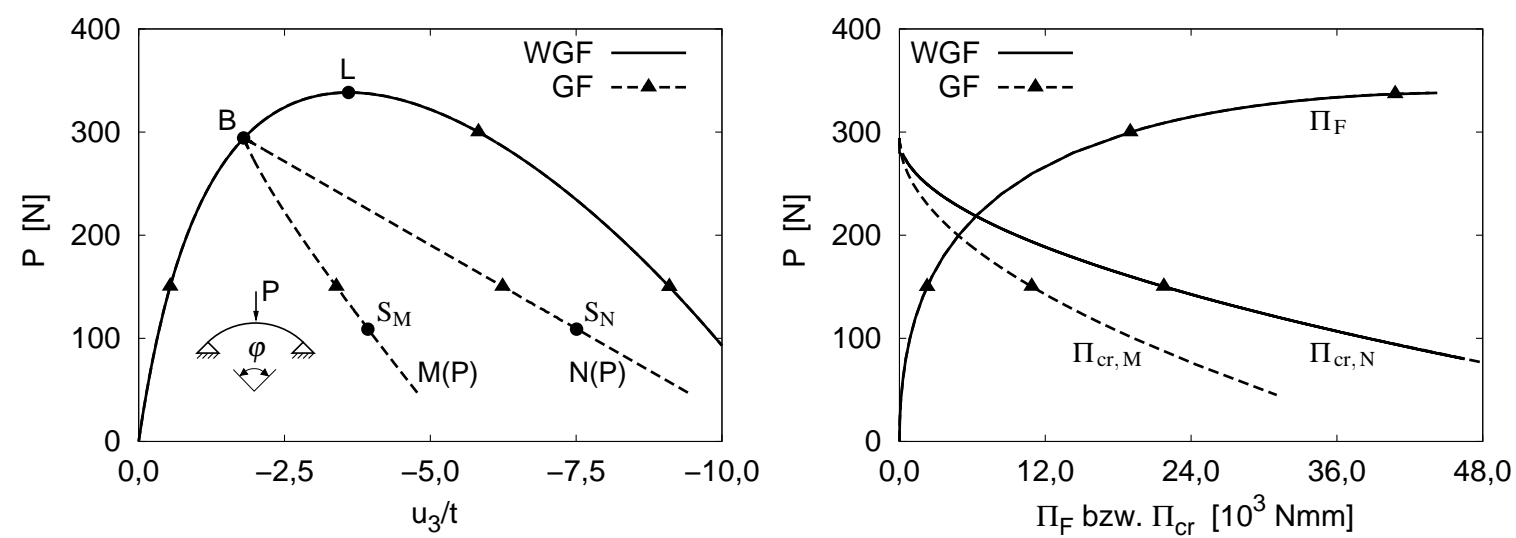

Bild 4.11: Einfluss der FE-Formulierung auf das Modellverhalten

Zur Diskretisierung des elastischen Potentials werden 16 Elemente mit biquadratischen Ansätzen verwendet. Der Last-Verformungs-Pfad einschließlich der Lastniveaus der singulären Punkte $B$ und $L$ sowie der Verlauf der Grundenergie stimmen für beide FE-Formulierungen mit der Referenzlösung [96, 151] gut überein. Dies gilt in der gemischten Formulierung auch für die kritischen Zustände $M$ und $N$ sowie für den Verlauf der Störenergie. Die Punkte $S_{M}$ und $S_{N}$ kennzeichnen Lastniveaus, unterhalb derer mit der inversen Vektoriteration Lösungen der Eigenwertprobleme (4.8) nicht zu ermitteln sind. Mit den LAPACK-Routinen [6] gelingt dies bis $P=45 \mathrm{~N}$. Für geringere Lastniveaus sind kritische Zustände nicht identifizierbar, da sich dann mehrere kritische Zustände in energetischer Hinsicht einander stark annähern [32]. In der Weggrößenformulierung sind kritische Zustände $N$ noch nicht direkt ermittelbar. Dies ist bisherigen Untersuchungen zufolge mit dem signifikant von der Belegung der Matrizen $\mathbf{K}_{1}$ und $\mathbf{K}_{2}$ abhängigen Spektrum der Eigenwerte zu begründen. Das Spektrum ist durch die Dominanz komplexer Eigenwerte geprägt, wodurch die Ermittlung reeller Eigenwerte erschwert wird. Dennoch kann anhand der Differenz der Verformungen zwischen primärem und sekundärem Last-Verformungs-Pfad gezeigt werden, dass Eigenwertproblem (4.30) erfüllt ist und Störenergie (4.35) die Empfindlichkeit des Systems gegenüber kinetischen Störungen korrekt beschreibt. 


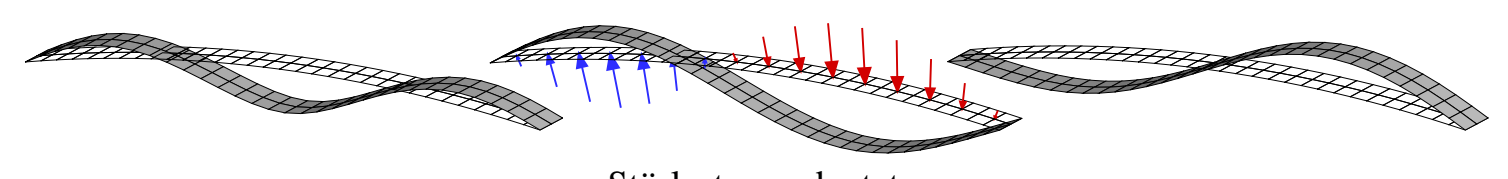

Störlast angedeutet

Bild 4.12: Verformungen infolge Grundbelastung und Störlast sowie Beuleigenform

Die Wirkung der in der gemischten Formulierung berechenbaren Störlast auf das Tragverhalten des Schalentragwerkes stellt Bild 4.12 heraus. Während im Vorbeulbereich die Verformungen symmetrisch zum Scheitelpunkt des Systems sind, bewirkt die zur Grundbelastung diskretisierungsbedingt nur annähernd orthogonale Störlast $\left(\Varangle\left(\mathbf{p}_{F}, \mathbf{p}_{p}\right)=89,5^{\circ}\right)$ einen unsymmetrischen Übergang in den Nachbeulbereich.

\subsection{Stabilitätstheoretische Klassifizierung}

Die Anwendungsmöglichkeiten des Störenergie-Konzeptes sind in Bild 4.13 aufgezeigt. Neben der Bewertung der Imperfektionsempfindlichkeit von Grundzuständen im Fall nicht anfänglicher Störungen liegt der Schwerpunkt der Anwendung des Störenergie-Konzeptes auf der Identifikation statischer und kinetischer Grenzlasten.

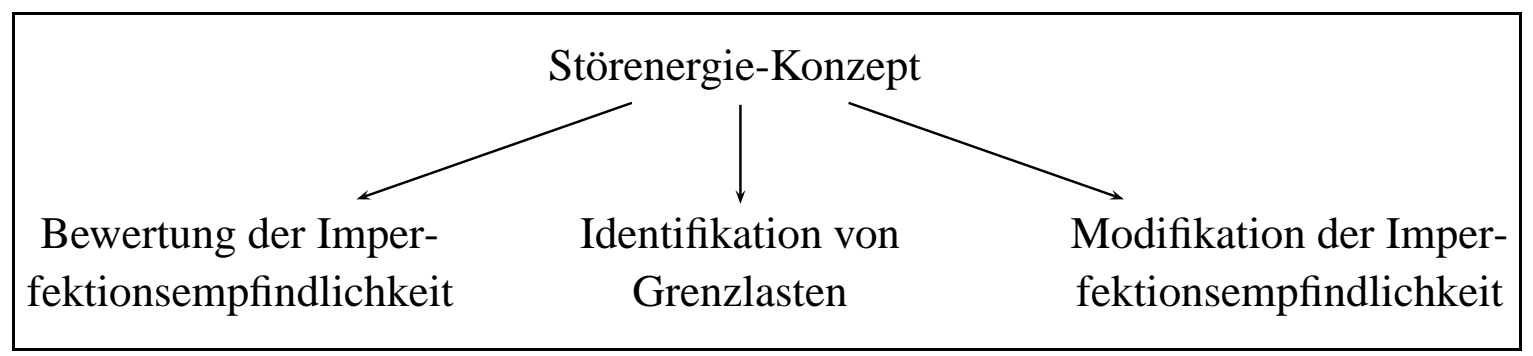

Bild 4.13: Anwendungsmöglichkeiten des Störenergie-Konzeptes

Die Bewertung der Imperfektionsempfindlichkeit im Fall anfänglicher Störungen und die in Abschnitt 8.3 vorgestellte lastniveauspezifische Modifikation der Imperfektionsempfindlichkeit erlauben weitere Aussagen zur Stabilität von Schalentragwerken und verdeutlichen die stabilitätstheoretische Bedeutung des Störenergie-Konzeptes.

Das Störenergie-Konzept ist gemäß den Ausführungen in den Kapiteln 3 und 4 wie folgt in die Theorie der Stabilität von Systemen einzuordnen: Das Störenergie-Konzept basiert auf der zweiten Methode nach Ljapunow und ermöglicht im Fall statischer Störungen die Identifikation von Grenzen der positiven Definitheit des zugrunde liegenden Ljapunow-Funktionals. Im Fall kinetischer Störungen ist ein Grundzustand als Attraktor zu interpretieren. Mit dem Störenergie-Konzept sind Grenzen des Einzugsgebietes dieses Attraktors beschreibbar, wobei die Anfangsbedingungen einer Bewegung und Störungen der Systemparameter berücksichtigt werden können.

Konventionelle Stabilitätskriterien basieren u.a. auf der Identifikation singulärer Punkte im primären Last-Verformungs-Pfad und reduzieren die Stabilitätsaussage auf die 
Feststellung von Stabilität oder Instabilität. Das Störenergie-Konzept hingegen ermöglicht eine Aussage darüber, wie stabil ein Grundzustand ist, und definiert für jeden Grundzustand eine Grenze zwischen Vor- und Nachbeulbereich. Die Störenergie erlaubt einen direkten quantitativen und anschaulichen Vergleich der Imperfektionsempfindlichkeit und Stabilität verschiedener Grundzustände und Beulfälle.

In methodischer Hinsicht ist Ähnlichkeit zwischen dem Störenergie-Konzept und dem Total-Potential-Energy-Approach [135] zu erkennen. Dieser Ansatz basiert aber nicht auf der Identifikation kritischer Zustände und ermöglicht folglich nur die Herleitung einer unteren und oberen Grenze der kinetischen Grenzlast. Die Lower-BoundTheory [26] sowie das Konzept der richtungsorientiert reduzierten Membranbeulung [161] basieren auf der Vernachlässigung bestimmter Membrananteile im elastischen Potential. Mit dem Störenergie-Konzept sind entsprechende Anteile für unterschiedliche Beulfälle näher quantifizierbar. Die Störenergie ist im Fall einer kinetischen Störung ein dem Stabilitätsgrad nach Leipholz [102] äquivalentes Maß für die Stabilität eines Grundzustandes. Die von Vielsack et al. [149] definierte Sensitivität basiert nicht auf der Identifikation kritischer Zustände und ist nicht proportional zur Störenergie. Die Ermittlung der statischen Grenzlast mit Hilfe der bezogenen Störenergie ist zwar formal ähnlich zu Methoden, die diese Grenzlast mittels Abminderungsfaktoren oder Ersatzimperfektionen beschreiben. Ein Vorteil ist jedoch die energetisch äquivalente Bewertung der Grenzlasten von Schalentragwerken. Die in Experimenten feststellbare eklatante Streuung der Grenzlasten ist mit dem Störenergie-Konzept nicht direkt, sondern erst mit der Definition des Referenzwertes der bezogenen Störenergie auf Basis von Experimenten oder Regelwerken indirekt zu berücksichtigen.

In algorithmischer Hinsicht ist das Störenergie-Konzept als spezielle Pfadwechselprozedur anzusehen, mit der ein Pfadwechsel ohne Existenz bzw. Identifikation von Verzweigungspunkten erfolgen kann. Eine Bewertung des Stabilitätsverhaltens im Nachbeulbereich ist mittels Pfadwechsels und erneuter Lösung der Eigenwertprobleme (4.8) möglich. Dabei sind die Anwendungsgrenzen der Schalentheorie zu beachten, die der numerischen Umsetzung des Störenergie-Konzeptes zugrunde liegt.

In mathematischer Hinsicht ist das Störenergie-Konzept eine Methode zur Bewertung der Stabilität der Lösung von Differentialgleichungssystemen. Der Störlast und der Störenergie sind dabei eine systemspezifische Bedeutung zuzuordnen. Das Störenergie-Konzept zeigt Ähnlichkeit zur aus der Mathematik bekannten Störungsrechnung. Die Ähnlichkeit beruht auf der Idee der Störung der Systemparameter. Der entscheidende Unterschied zwischen dem Störenergie-Konzept und der Störungsrechnung liegt im Vorgehen zur Ermittlung der Antwort eines Systems auf Störungen. 


\section{Statische Grenzlasten von Kreiszylinderschalen}

Die Imperfektionsempfindlichkeit und die Grenzlasten verschiedenartig gelagerter und belasteter Kreiszylinderschalen werden nachfolgend mit dem Störenergie-Konzept bewertet. Die Untersuchungen umfassen Kreiszylinderschalen mit isotropem und anisotropem Materialverhalten.

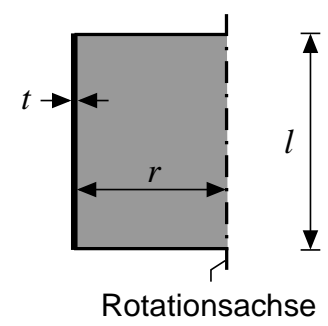

\subsection{Konstante Axiallast}

Für Kreiszylinderschalen unter konstanter Axiallast ist im Fall linear elastischen, isotropen Materialverhaltens die klassische Beullast nach Timoshenko [143] mit

$$
p_{c l}=\frac{E}{\sqrt{3\left(1-v^{2}\right)}} \frac{t^{2}}{r}
$$

berechenbar. Die klassische Beullast beschreibt nur eine obere Grenze für die Beullast von Kreiszylinderschalen, da Störungen des Membranspannungszustandes und geometrische Nichtlinearitäten nicht berücksichtigt werden. Die Analyse dieser Einflüsse erfolgt für drei verschiedenartig gelagerte Kreiszylinderschalen, wobei radial unverschiebliche Ränder auch in Umfangsrichtung unverschieblich sind, vgl. Bild 5.1. Die Materialparameter sind zu $E=2,06 \cdot 10^{5} \mathrm{~N} / \mathrm{mm}^{2}$ und $\mathrm{v}=0,30$ festgelegt.
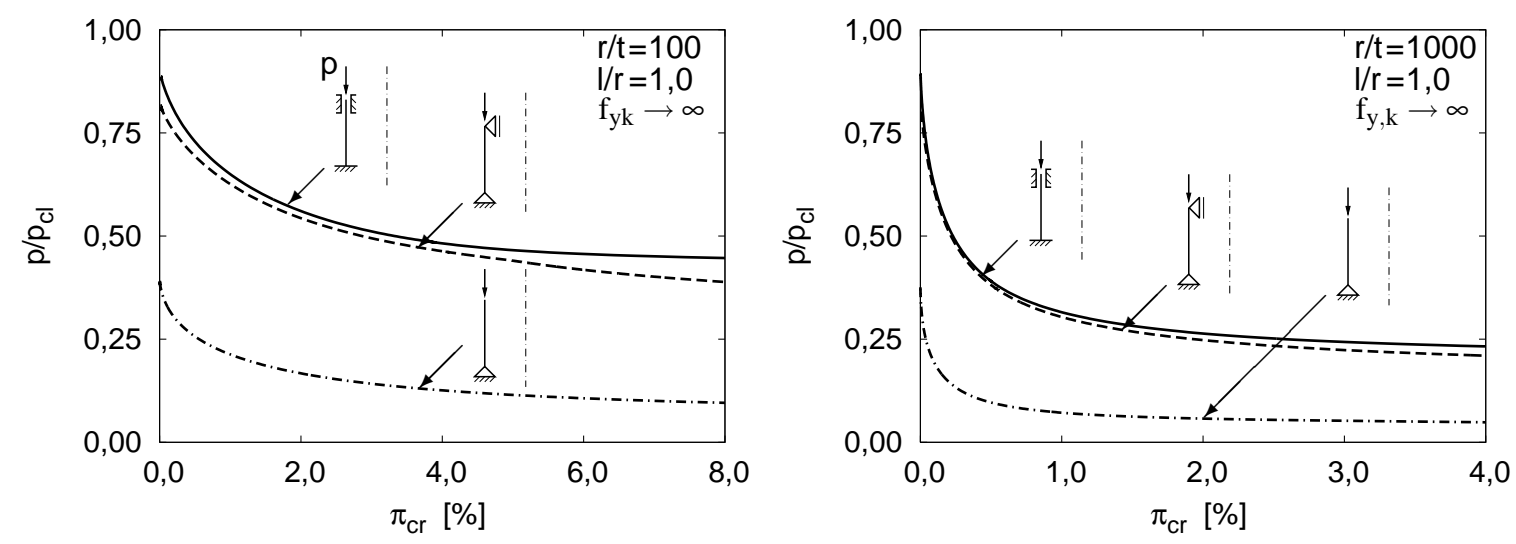

Bild 5.1: Imperfektionsempfindlichkeit vs. Beulfall

Die auf die klassische Beullast bezogene Beullast ist nahezu unabhängig von der Schlankheit und für beidseitig eingespannte Kreiszylinderschalen geringfügig grö- 
Ber als für am belasteten Rand wölbfrei gelagerte Kreiszylinderschalen. Eine im Vergleich zu diesen Beulfällen deutlich geringere bezogene Beullast besitzen am belasteten Rand freie Kreiszylinderschalen. Der Verlauf der bezogenen Störenergie ist, wie beide Diagramme in Bild 5.1 erkennen lassen, unabhängig von den Randbedingungen durch zwei Grenzwerte gekennzeichnet. Im Bereich des ersten singulären Punktes im Last-Verformungs-Pfad strebt die bezogene Störenergie gegen null und im Bereich des Nachbeulminimums gegen unendlich. Zwischen beidseitig eingespannten und am belasteten Rand wölbfrei gelagerten Kreiszylinderschalen ist der Unterschied in der bezogenen Störenergie für hohe Lastniveaus gering und im Bereich des Nachbeulminimums maximal. Mit zunehmender Schlankheit verringern sich der Unterschied und die bezogene Störenergie merklich. Daher werden in den weiteren Untersuchungen beidseitig eingespannte Kreiszylinderschalen nicht berücksichtigt. Für am belasteten Rand freie Kreiszylinderschalen ist die Differenz im Lastniveau zwischen erstem singulären Punkt und Nachbeulminimum geringfügig kleiner als für am belasteten Rand wölbfrei gelagerte Kreiszylinderschalen. Der Verlauf der bezogenen Störenergie ist für beide Beulfälle aber sehr ähnlich. Somit weisen die Beulfälle eine ähnliche Imperfektionsempfindlichkeit auf.
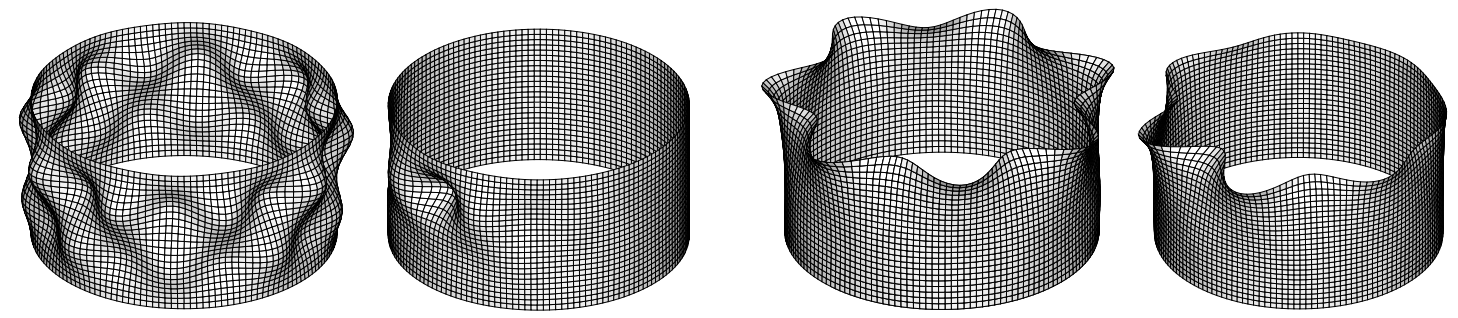

Bild 5.2: Beuleigenform und Verformungen infolge Störlast vs. Beulfall

Beuleigenform und Verformungen infolge Störlast sind in Bild 5.2 für eine am belasteten Rand wölbfrei gelagerte bzw. freie Kreiszylinderschale mit der Schlankheit $r / t=100$ dargestellt. In Abhängigkeit vom Beulfall erfasst die Beuleigenform die gesamte Kreiszylinderschale oder nur deren oberen Bereich. Die Störlast ist, wie ein Vergleich mit Bild 5.20 erwarten lässt, für beide Beulfälle orthogonal zur Grundbelastung. Den dominierenden Anteil der Störlast repräsentieren Kraftgrößen. Die Verformungen infolge Störlast unterscheiden sich signifikant von der entsprechenden Beuleigenform. Für die am belasteten Rand wölbfrei gelagerte Kreiszylinderschale sind die Verformungen infolge Störlast durch eine lokale Einzelbeule im Bereich des belasteten Randes charakterisiert. Die Störenergie ist lokal konzentriert. Dagegen sind für die am belasteten Rand freie Kreiszylinderschale sowohl die Verformungen infolge Störlast als auch die Störenergie unmittelbar am oberen Rand konzentriert und in Umfangsrichtung schwächer abklingend. Für beide Beulfälle sind die Verformungen infolge Störlast von dem Lastniveau und der Schlankheit abhängig.

Statische Grenzlasten sind für beide Beulfälle mit Hilfe des Referenzwertes der be- 
zogenen Störenergie festzulegen und in der Regel auf die klassische Beullast zu beziehen. Für am belasteten Rand freie Kreiszylinderschalen ist dem Autor eine analytisch ermittelte Gleichung zur Berechnung der klassischen Beullast nicht bekannt. Die Beullast ist mittels linearer Beulanalyse zu $p_{c l, f}=0,385 p_{c l}$ abschätzbar und besitzt eine geringfügig größere Abhängigkeit von der Schlankheit als die klassische Beullast (5.1), wird aber dennoch nachfolgend zur Darstellung der Grenzlasten von am belasteten Rand freien Kreiszylinderschalen verwendet.
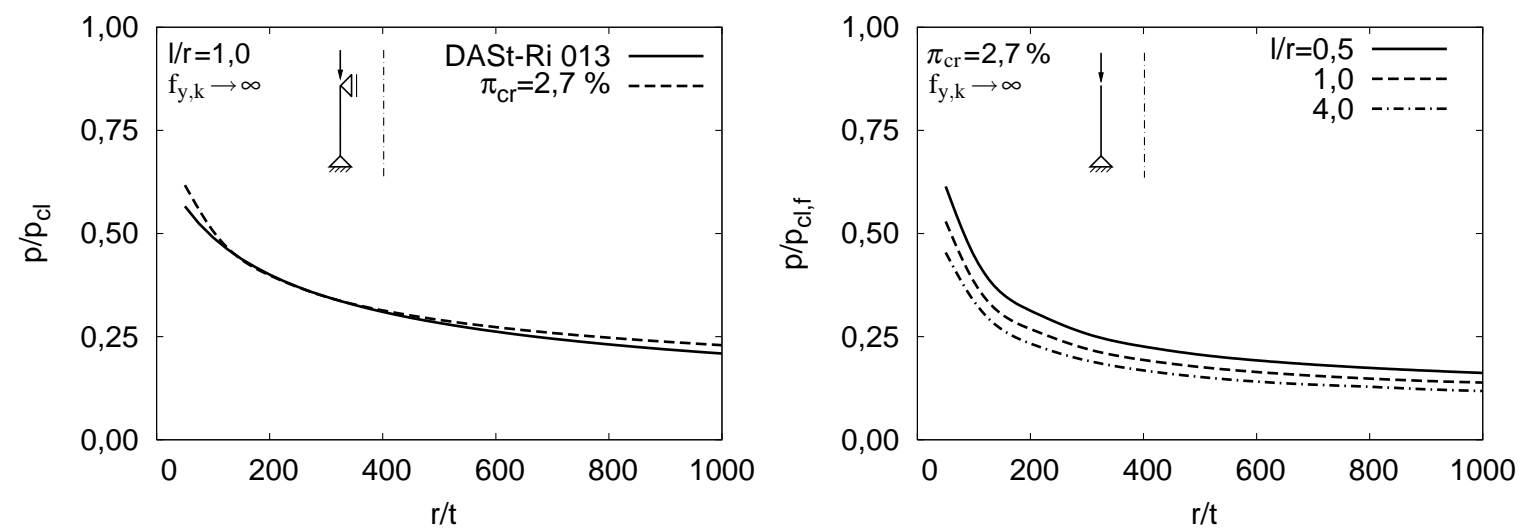

Bild 5.3: Grenzlasten nach DASt-Richtlinie 013 und Störenergie-Konzept

Für am belasteten Rand wölbfrei gelagerte Kreiszylinderschalen zeigt Bild 5.3 links die Grenzlasten gemäß DASt-Richtlinie 013 und gemäß Referenzwert der bezogenen Störenergie. Auf der guten Übereinstimmung der Grenzlasten basiert die Definition des Referenzwertes [87]. Für am belasteten Rand freie Kreiszylinderschalen mit unterschiedlichem Längen-Radius-Verhältnis $l / r$ sind in Bild 5.3 rechts die Grenzlasten gemäß Referenzwert der bezogenen Störenergie angegeben. Mit abnehmendem Längen-Radius-Verhältnis steigen der Einfluss der Randbedingungen und die Grenzlast an, während sich die Imperfektionsempfindlichkeit für ein definiertes Lastniveau verringert. Für die berücksichtigten Längen-Radius-Verhältnisse kann die Grenzlast bei gleicher Schlankheit um bis zu 35\% variieren. Die für elastisches Materialverhalten ermittelten und auf die Streckgrenze $\mathrm{f}_{\mathrm{y}, \mathrm{k}}=360 \mathrm{~N} / \mathrm{mm}^{2}$ bezogenen Grenzlasten sind in Bild 5.4 dem Abminderungsfaktor $\kappa_{2}$ nach DIN 18800 gegenübergestellt.
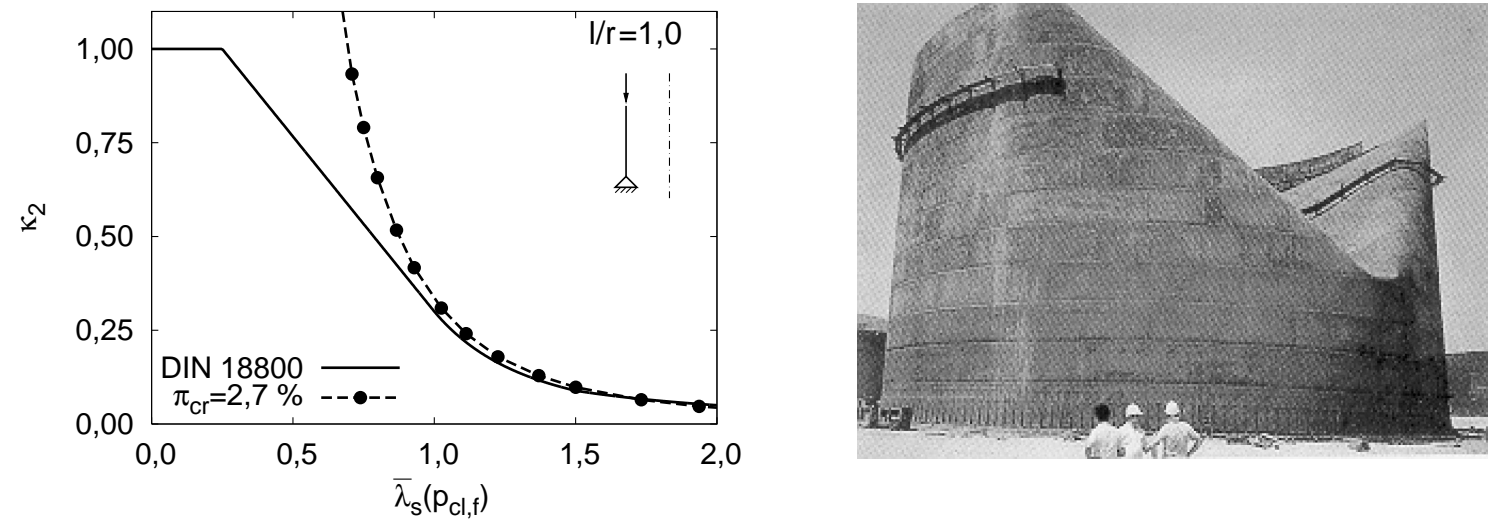

Bild 5.4: Grenzlasten sowie ein Schadensfall (Bildquelle unbekannt) 
Für bezogene Schlankheitsgrade $\bar{\lambda}_{s}>0,9$ stimmen die ermittelten bezogenen Grenzlasten und der Abminderungsfaktor nach DIN 18800 gut überein, obwohl dieses Regelwerk wie auch die ECCS-Recommendations nicht für am belasteten Rand freie Kreiszylinderschalen gelten. Für elastisch-plastisches Materialverhalten sind indes weitere Untersuchungen erforderlich, deren Notwendigkeit der in Bild 5.4 dargestellte Schadensfall und die Ausführungen in Abschnitt 6.3 verdeutlichen.

\subsection{Kombinierte Belastungen}

Kreiszylinderschalen können gleichzeitig durch eine axiale Randlast $p$ und eine ebenfalls in Axialrichtung wirkende Flächenlast $p_{\mathrm{v}}$ beansprucht werden. Der Einfluss dieser Belastungen auf das Tragverhalten wird in Bild 5.5 für eine am belasteten Rand wölbfrei gelagerte Kreiszylinderschale herausgestellt, wobei die Summe $p_{s}=p+p_{\mathrm{v}} 1$ beider Belastungen der Darstellung der bezogenen Störenergie zugrunde liegt.

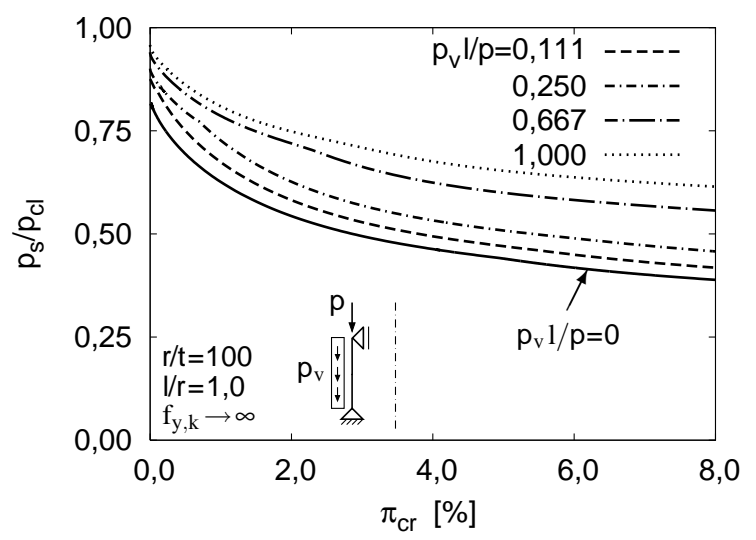

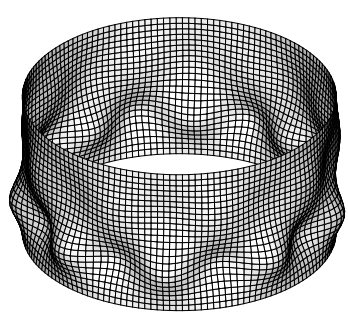

Beuleigenform

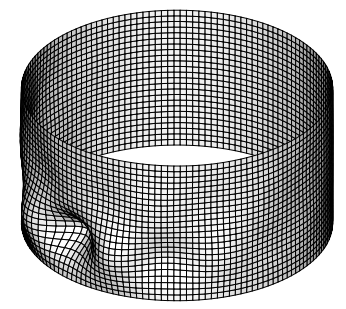

Verformungen infolge Störlast

Bild 5.5: Tragverhalten vs. Axialbelastung

Mit ansteigendem Anteil der Flächenlast an der gesamten Belastung nimmt die Beullast zu und die Imperfektionsempfindlichkeit für ein definiertes Lastniveau ab. Gleichzeitig steigt die Grenzlast gemäß Referenzwert der bezogenen Störenergie oftmals stärker an als die Beullast. Die Beuleigenform ist bereits bei einem geringen Anteil der Flächenlast an der gesamten Belastung am unteren Rand konzentriert, während Störlast und zugehörige Verformungen für hohe Lastniveaus am unteren und für niedrige Lastniveaus am oberen Rand lokalisiert sind. Den Übergang zwischen beiden Positionen verdeutlicht die Diskontinuität in der Steigung des Verlaufes der bezogenen Störenergie. Beide Positionen der Störlast sind mit dem Maximum der Membrandruckkraft und der Wölbbehinderung am unteren Rand zu begründen.

Kreiszylinderschalen sind auch kombinierten Axial- und Radialbelastungen ausgesetzt. Das Verhältnis beider Belastungen und die Art der Radialbelastung können das Tragverhalten signifikant beeinflussen. Der Einfluss wird für am belasteten Rand wölbfrei gelagerte bzw. freie Kreiszylinderschalen untersucht, wobei neben der in 
Axialrichtung wirkenden Randlast $p$ vier verschiedene Radialbelastungen berücksichtigt werden. Hierzu zählen neben konstantem Radialaußen- und -innendruck auch teilweiser Radialinnendruck und Radialbelastung infolge Windes. Für die ersten drei Radialbelastungen sind die betragsmäßig maximalen Lastordinaten identisch, wohingegen für die Radialbelastung infolge Windes der Beiwert $\delta$ nach DIN 18800 einbezogen wird. Somit ist diese Belastung von der Schlankheit, den Randbedingungen und dem Längen-Radius-Verhältnis abhängig und die Drucklast im Staupunkt betragsmäßig größer als die maximale Lastordinate der anderen Radialbelastungen.

Für eine am belasteten Rand wölbfrei gelagerte Kreiszylinderschale zeigt Bild 5.6 die Abhängigkeit der Beullast, der Imperfektionsempfindlichkeit und der Grenzlast vom Belastungsverhältnis $\eta$ und von der Art der Radialbelastung $p_{n}$ auf.
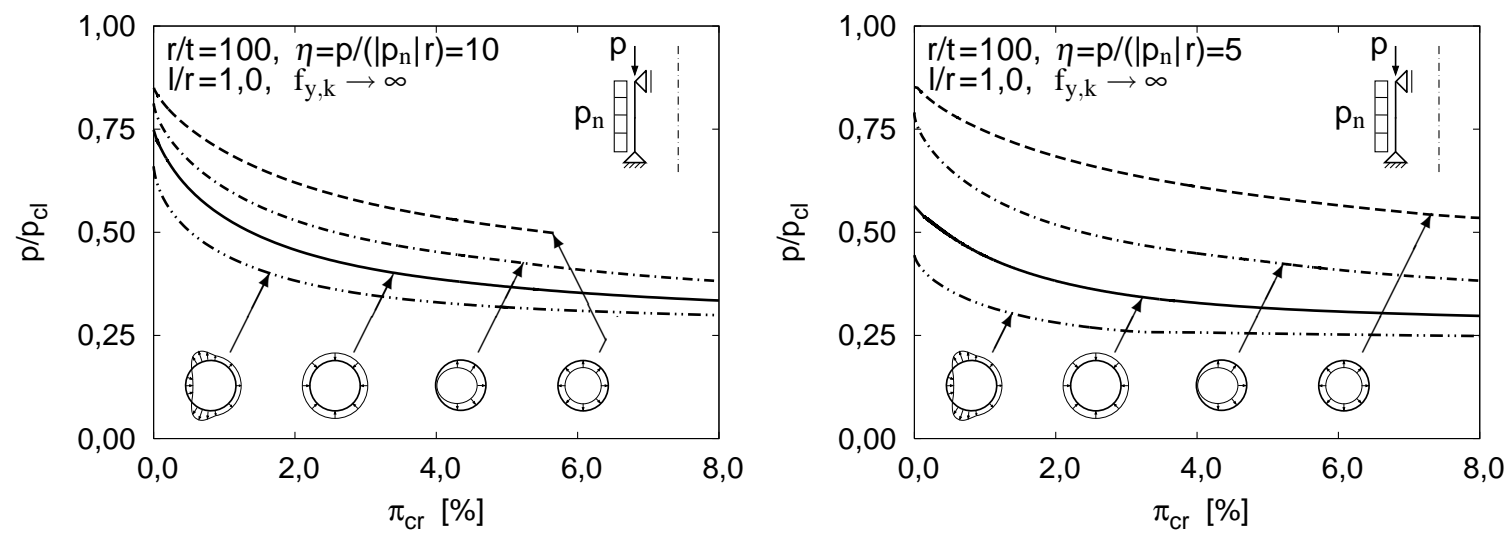

Bild 5.6: Tragverhalten vs. Axial- und Radialbelastung

Die Beullast ist für konstanten Radialinnendruck aufgrund dessen stabilisierender Wirkung am größten. Dagegen ist die Beullast für teilweisen Radialinnendruck etwas und für konstanten Radialaußendruck deutlich geringer. Die kleinste Beullast ist bei Radialbelastung infolge Windes zu erwarten. Die Differenz zwischen den Beullasten ist vom Belastungsverhältnis abhängig und wird mit ansteigender Radialbelastung größer. Gleichzeitig wird die Grenzlast gemäß Referenzwert der bezogenen Störenergie stärker von der Art der Radialbelastung beeinflusst. Die Abhängigkeit der Grenzlast von der Schlankheit und den Randbedingungen stellt Bild 5.7 heraus.

Mit zunehmender Schlankheit wird die Grenzlast der analysierten Kreiszylinderschalen kleiner. Dabei ist für am belasteten Rand freie Kreiszylinderschalen die Grenzlast wesentlich geringer und der Einfluss der Radialbelastung geringfügig kleiner als bei am belasteten Rand wölbfrei gelagerten Kreiszylinderschalen. Für verschiedene Belastungsverhältnisse ist die Grenzlast in Bild 5.8 angegeben, wobei $p_{n, c l}$ die klassische Beullast einer Kreiszylinderschale unter Radialdruck darstellt [31].

Für zunehmenden konstanten Radialinnendruck erfährt die Grenzlast eine signifikante Erhöhung. Dagegen ist die Grenzlast nahezu unabhängig vom teilweisen Radial- 

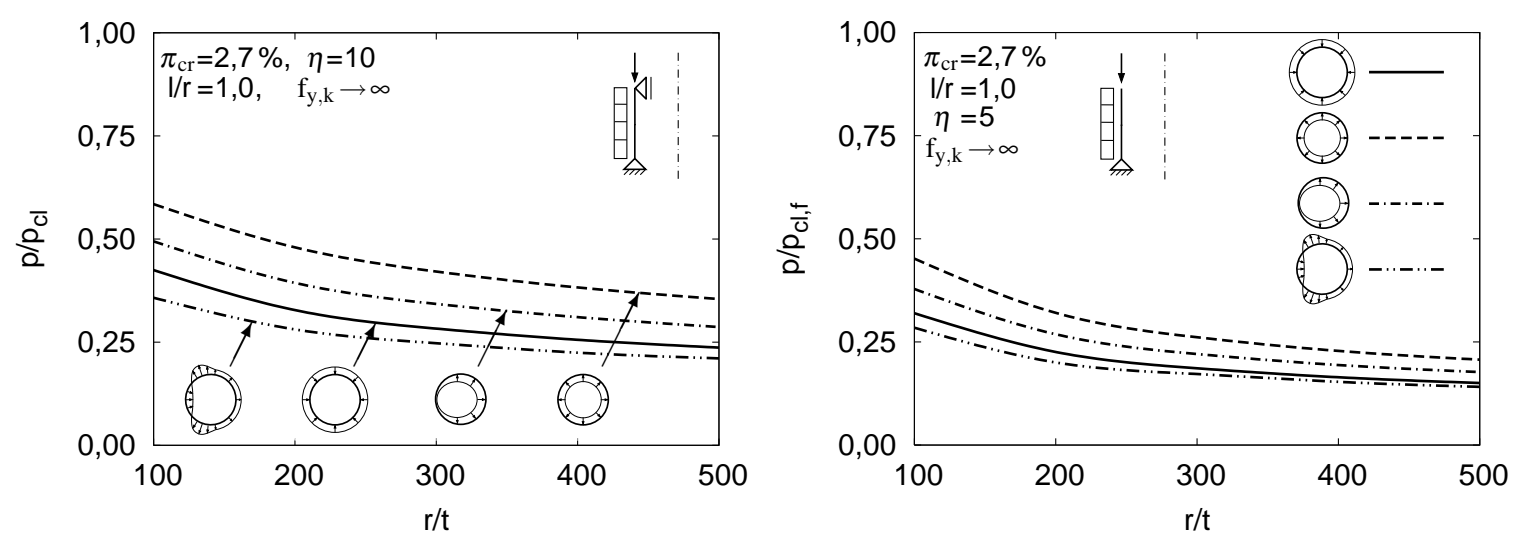

Bild 5.7: Grenzlast vs. Belastung, Schlankheit und Randbedingungen
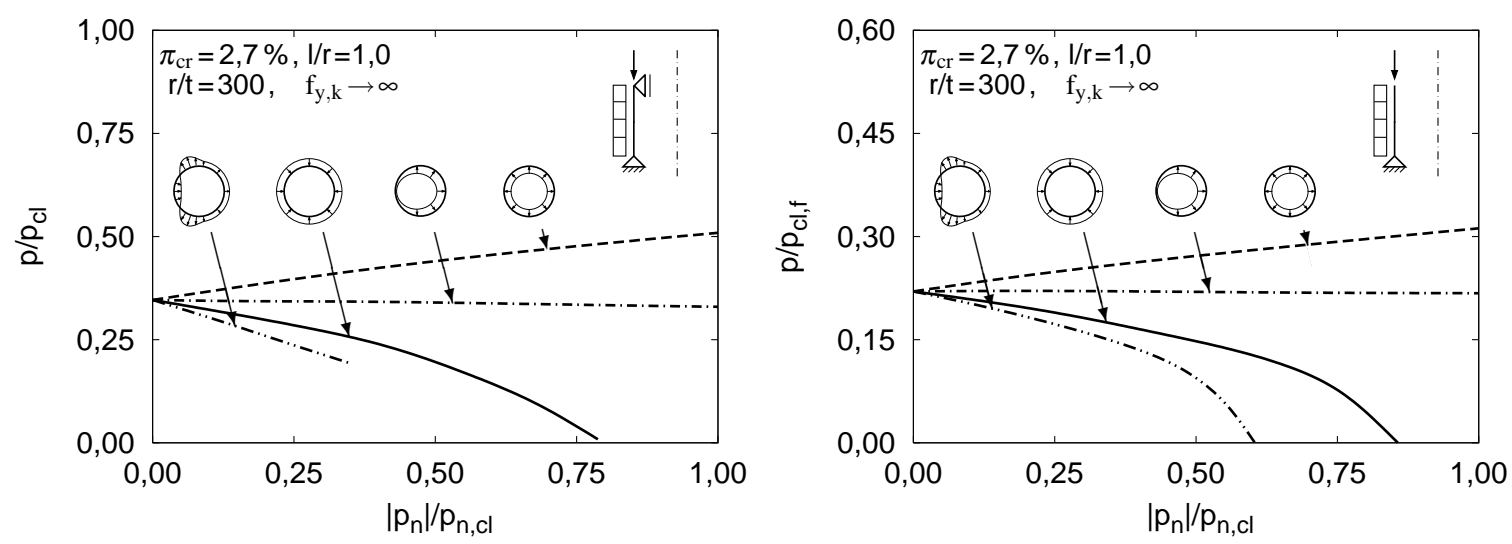

Bild 5.8: Grenzlast vs. Axial- und Radialbelastung

innendruck, da Störlast und zugehörige Verformungen im Bereich verschwindender Radialbelastung konzentriert sind. Für die Radialbelastung infolge Windes verringert sich die Grenzlast mit abnehmendem Belastungsverhältnis stärker als für konstanten Radialaußendruck, da die Kreiszylinderschale infolge Windes bereits im Grundzustand nicht rotationssymmetrisch deformiert ist. Ferner sind die Verformungen infolge Störlast im Bereich des Staupunktes konzentriert, wobei deren Ausdehnung in Meridianrichtung von der Größe der Radialbelastung abhängig ist. Im Vergleich zum konstanten Radialaußendruck bewirken Radialbelastungen, die mit dem ersten bzw. zweiten Fourier-Reihenglied beschreibbar sind, zumindest für Belastungsverhältnisse größer zehn nur eine geringfügige Änderung der Imperfektionsempfindlichkeit.

Die für kombinierten Axial- und konstanten Radialinnendruck angegebenen Grenzlasten setzen voraus, dass der stabilisierende Radialinnendruck während des gesamten für die Bewertung der Stabilität relevanten Zeitraumes in vollem Umfang wirkt.

\subsection{Einfluss von Störungen, Temperatur und Aussteifungen}

Störungen können das Tragverhalten von Schalentragwerken destabilisieren und sind entsprechend den Ausführungen in Abschnitt 3.6 als anfänglich oder nicht anfäng- 
lich wirkend zu klassifizieren. Anfängliche Störungen sind hinsichtlich ihres Einflusses auf die Beul- und die Grenzlast, die Grundenergie und die störungsbedingte Änderung der bezogenen Störenergie, ausgedrückt nach Gl. (4.29) mit $\Delta \pi_{c r}^{I m p}$, bewertbar. Den Einfluss anfänglicher geometrischer Störungen bzw. Imperfektionen auf das Tragverhalten einer axialgedrückten Kreiszylinderschale zeigt Bild 5.9 auf.
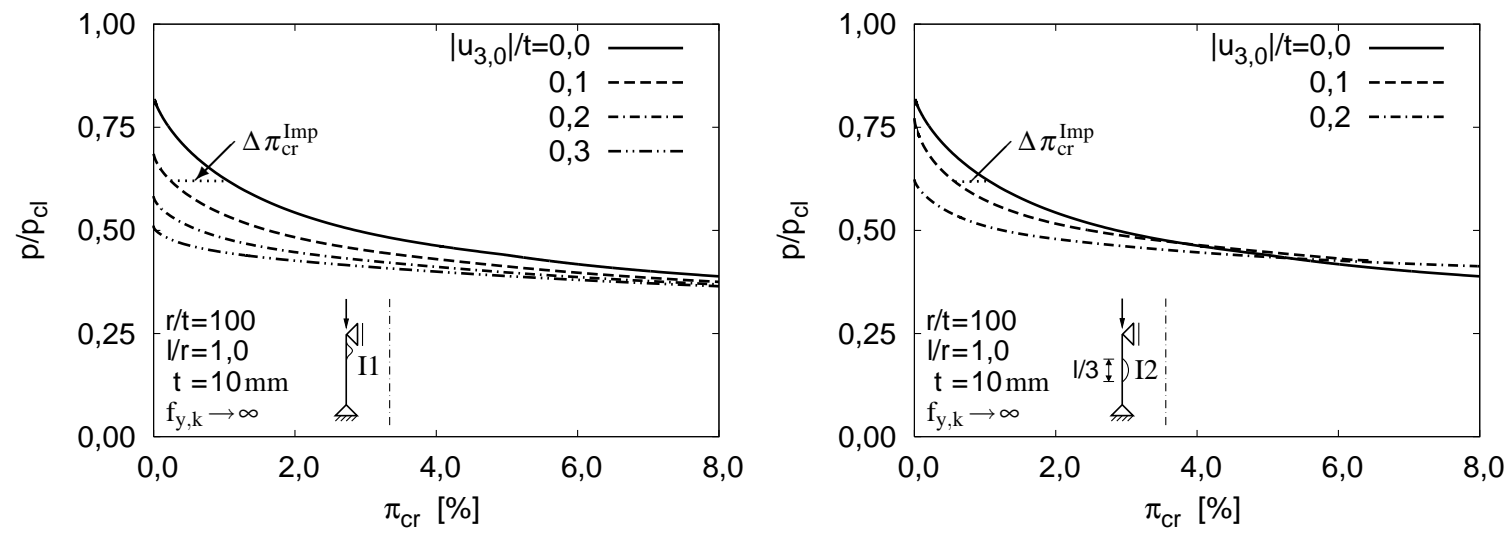

Bild 5.9: Imperfektionsempfindlichkeit vs. anfängliche Störung

Die Imperfektion I1 ist affin zu den Verformungen infolge Störlast auf dem Lastniveau $p / p_{c l}=0,78$ und die ringwulstförmige, im mittleren Drittel der Kreiszylinderschale angeordnete Imperfektion I2 in Meridianrichtung mit einer Sinus-Halbwelle beschrieben. Für die gleiche Amplitude $u_{3,0} / t$ verringert Imperfektion I1 die Beullast und die Grundenergie stärker als Imperfektion I2, vgl. [127]. Beuleigenform und Verformungen infolge Störlast sind jeweils im Bereich der Imperfektion konzentriert, wobei eine lokale Einzelbeule die Verformungen infolge Störlast charakterisiert. Für die nicht rotationssymmetrische Imperfektion I1 ist die Grenzlast kleiner und die imperfektionsbedingte Änderung der bezogenen Störenergie größer als für Imperfektion I2 gleicher Amplitude. Die Änderung der bezogenen Störenergie ist für Imperfektion 2 bereichsweise negativ und bringt somit deren lastniveauspezifisch stabilisierende Wirkung zum Ausdruck. Bemerkenswert ist allgemein die bereits bei kleinen Amplituden erkennbare signifikante Änderung des Tragverhaltens [81, 151, 2].

Regelwerke fordern bei der Ermittlung der Grenzlast die Einhaltung von Grenzwerten hinsichtlich der Tiefe von Vorbeulen und der Unrundheit von Kreiszylinderschalen. Für Imperfektion I1 mit der Amplitude $u_{3,0} / t=0,31$ und Imperfektion I 2 mit der Amplitude $u_{3,0} / t=0,35$ entspricht die Beullast der imperfekten Kreiszylinderschale der am perfekten System ermittelten Grenzlast, womit Regelungen verschiedener Regelwerke zur Tiefe von Vorbeulen eingehalten sind. Die Amplitude liegt für eine am belasteten Rand freie Kreiszylinderschale gleicher Schlankheit und Wanddicke, deren Imperfektion affin zu den Verformungen infolge Störlast auf dem Lastniveau $p / p_{c l}=0,30$ ist, mit $u_{3,0} / t=0,90$ deutlich über den Grenzwerten der Regelwerke. Für zwei Beulfälle ist in Bild 5.10 die zum Referenzwert der bezogenen Störenergie korrespondierende und auf die Messlänge $l_{g x}$ bezogene Amplitude der Verformungen 
infolge Störlast dem Grenzwert $U_{0, \max }$ nach EC 3 bzw. DIN 18800 gegenübergestellt. Für beide Beulfälle ist der Grenzwert kleiner als die bezogene Amplitude und nimmt die Differenz zwischen beiden Werten mit ansteigender Schlankheit zu. Daher wären die Amplituden der Verformungen infolge Störlast zu skalieren, um mit diesen als anfängliche Imperfektion die Grenzlasten der Regelwerke zu erreichen, vgl. Bild 6.21. Dennoch sind diese Verformungen als eine ungünstige Imperfektion anzusehen. Für zukünftige Entwicklungen der Regelwerke erscheint es sinnvoll, den Grenzwert für die Tiefe von Vorbeulen detaillierter in Abhängigkeit vom Beulfall festzulegen.
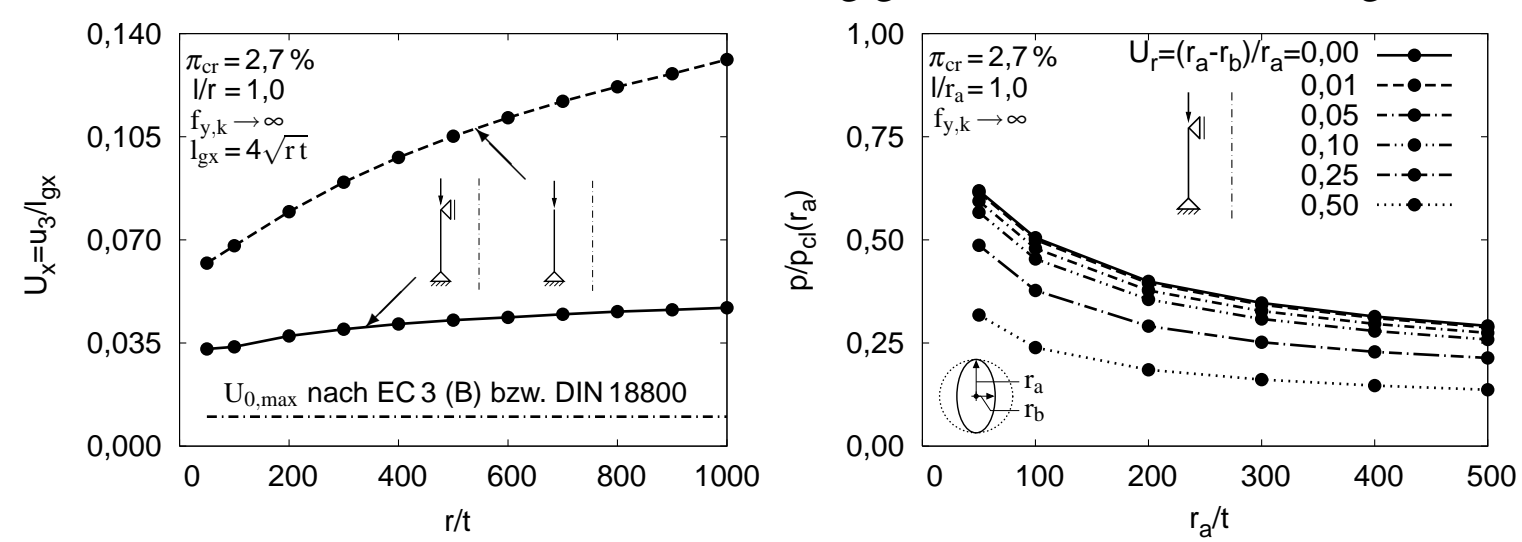

Bild 5.10: Verformungen infolge Störlast und Grenzlast vs. Unrundheit

Mit zunehmender Unrundheit einer Kreiszylinderschale werden Beul- und Grenzlast kleiner und die Imperfektionsempfindlichkeit für ein definiertes Lastniveau größer. Für am belasteten Rand wölbfrei gelagerte Kreiszylinderschalen mit $r_{a} / t=100$ und unterschiedlicher elliptischer Unrundheit, die sich jeweils über die gesamte Meridianlänge erstreckt, sind in Bild 5.11 die Verformungen infolge Störlast dargestellt.
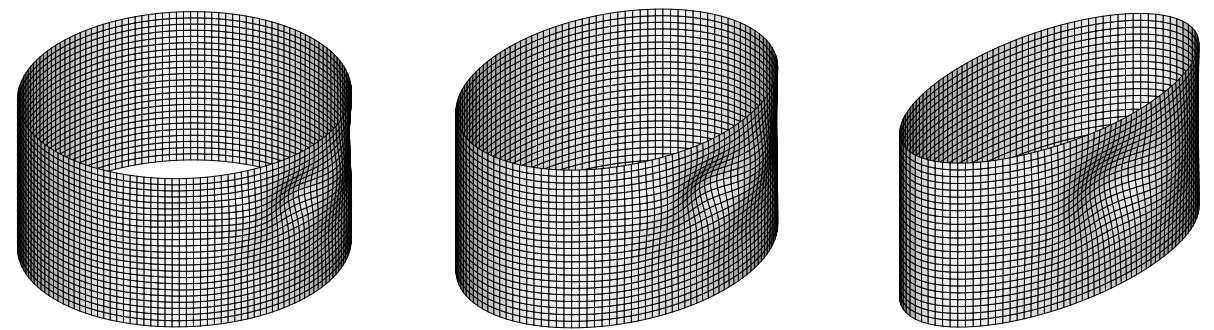

Bild 5.11: Verformungen infolge Störlast bei Unrundheiten $U_{r}=0,05,0,25$ und 0,50

Die Verformungen sind im Bereich der geringsten Flächenkrümmung konzentriert und mit zunehmender Unrundheit durch eine größere Ausdehnung in Meridian- und Umfangsrichtung charakterisiert. Der Einfluss einer Unrundheit auf die Grenzlast ist nur geringfügig von der Schlankheit abhängig, vgl. Bild 5.10. Die sehr geringen Unterschiede zwischen den Grenzlasten bei $U_{r}=0,00$ bzw. 0,01 bestätigen die Regelungen des EC 3, die für den untersuchten Beulfall den Grenzwert $U_{r, \max }=0,01$ vorschreiben. Ein Vergleich der Grenzlasten mit denen nach Bild 5.3 verdeutlicht, dass für axialgedrückte Kreiszylinderschalen die Randbedingungen am belasteten Rand die Grenzlast stärker beeinflussen können als eine ausgeprägte Unrundheit. 
Der Einfluss nicht anfänglicher Störungen auf die Stabilität eines Grundzustandes wird für eine am belasteten Rand in Umfangsrichtung nicht gelagerte, in Radialrichtung aber elastisch gebettete Kreiszylinderschale untersucht. Der Bettungsmodul $K_{B}$ erfährt auf dem Lastniveau $p / p_{c l}=0,59$ in Umfangsrichtung konstante Änderungen $\Delta K_{B}$. Die mit diesen Störungen in der Umgebung des Grundzustandes $F$ einhergehenden Änderungen der Topologie der Energiefläche zeigt Bild 5.12 auf.

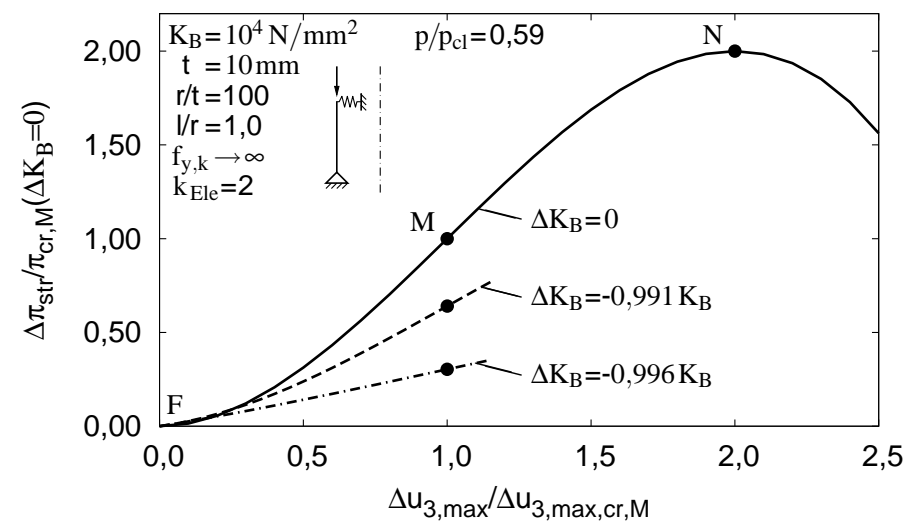

Bild 5.12: Stabilität eines Grundzustandes vs. nicht anfängliche Störung

Für das perfekte System wird $\pi_{c r, M}=0,16 \%$ ermittelt. Erst eine beträchtliche Verringerung des Bettungsmoduls erhöht die Imperfektionsempfindlichkeit des Grundzustandes merklich. Der Abstand zwischen Grundzustand und kritischem Zustand $M$ wird, wie aus der normierten Darstellung zwar nicht ersichtlich, mit zunehmender Störung kleiner, vgl. Bild 3.4. Gleichzeitig nähern sich die Verformungen infolge Störlast der Beuleigenform an. Für hinreichend große Störungen wird der Grundzustand instabil. Kritische Zustände $N$ können mit Eigenwertproblem (4.28) nicht ermittelt werden, da die Voraussetzungen für dessen Anwendung nicht erfüllt sind.

Für baupraktisch relevante Temperaturänderungen ist die Temperaturabhängigkeit der Materialparameter oft vernachlässigbar. Somit liegt der wesentliche Effekt von Temperaturänderungen in der Beeinflussung der Randstörungen. Für zwei am belasteten Rand wölbfrei gelagerte Kreiszylinderschalen aus Stahl verdeutlicht Bild 5.13 den Einfluss zweier gleichmäßiger Temperaturänderungen $\Delta T$ auf das Tragverhalten.
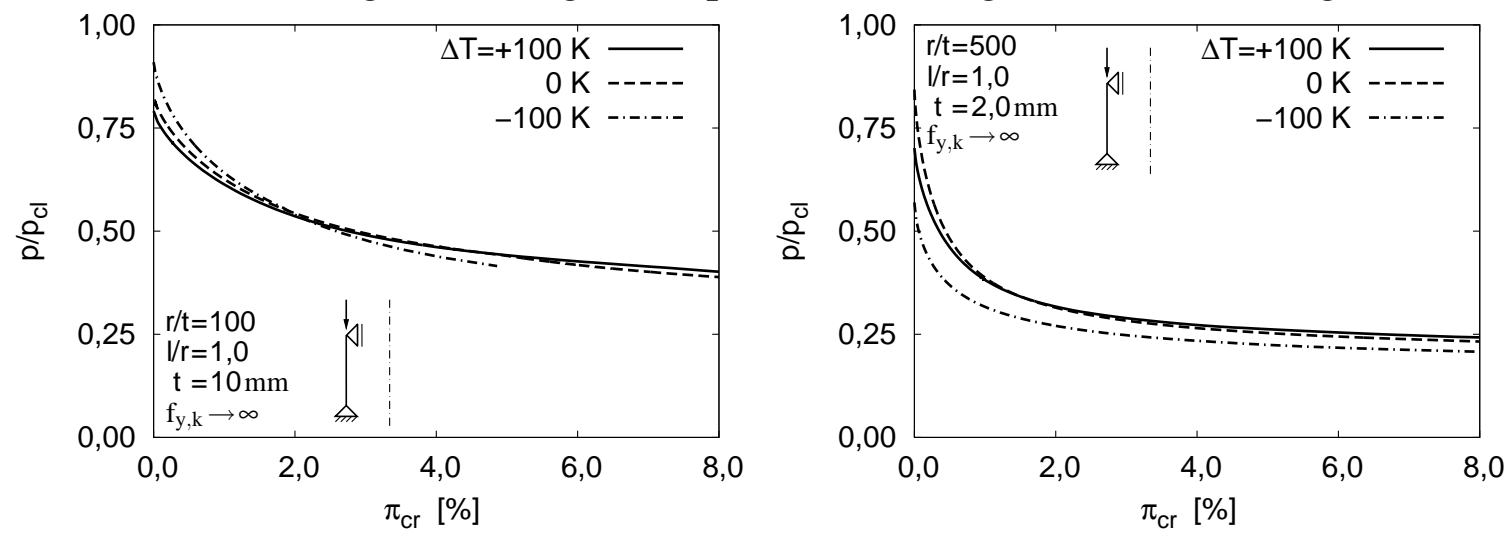

Bild 5.13: Tragverhalten vs. gleichmäßige Temperaturänderung 
Randstörungen treten an beiden Rändern auf. Bei der gedrungenen Kreiszylinderschale bewirken Axiallast und Temperaturänderung Randstörungen annähernd gleicher Größe. Beullast und Steigung des Verlaufes der bezogenen Störenergie werden bei Temperaturabnahme größer. Die Grenzlast wird nur geringfügig von den Temperaturänderungen beeinflusst. Bei der mittelschlanken Kreiszylinderschale sind die Randstörungen maßgeblich durch die Temperaturänderungen bedingt. Für beide Temperaturänderungen nimmt die Beullast ab, wohingegen sich die Grenzlast bei Temperaturzunahme kaum ändert und bei Temperaturabnahme deutlich verringert. Für am belasteten Rand freie Kreiszylinderschalen treten Randstörungen nur am gelagerten Rand auf. Daher beeinflussen Temperaturänderungen die Grenzlast kaum.

Aussteifungen sind hinsichtlich Steifigkeit und Anordnung zu unterscheiden. Nachfolgend wird exemplarisch der Einfluss ringförmiger Aussteifungen unendlich großer Steifigkeit, die mit Verschiebungsfesthaltungen in Radialrichtung modelliert sind, auf die Imperfektionsempfindlichkeit einer axialgedrückten Kreiszylinderschale untersucht. Eine hinsichtlich der Beeinflussung des Tragverhaltens günstige Anordnung der Aussteifungen in Meridianrichtung ist anhand der für einen Grundzustand und den zugehörigen kritischen Zustand $M$ in Bild 5.14 dargestellten radialen Verformungen der nicht ausgesteiften Kreiszylinderschale abschätzbar.
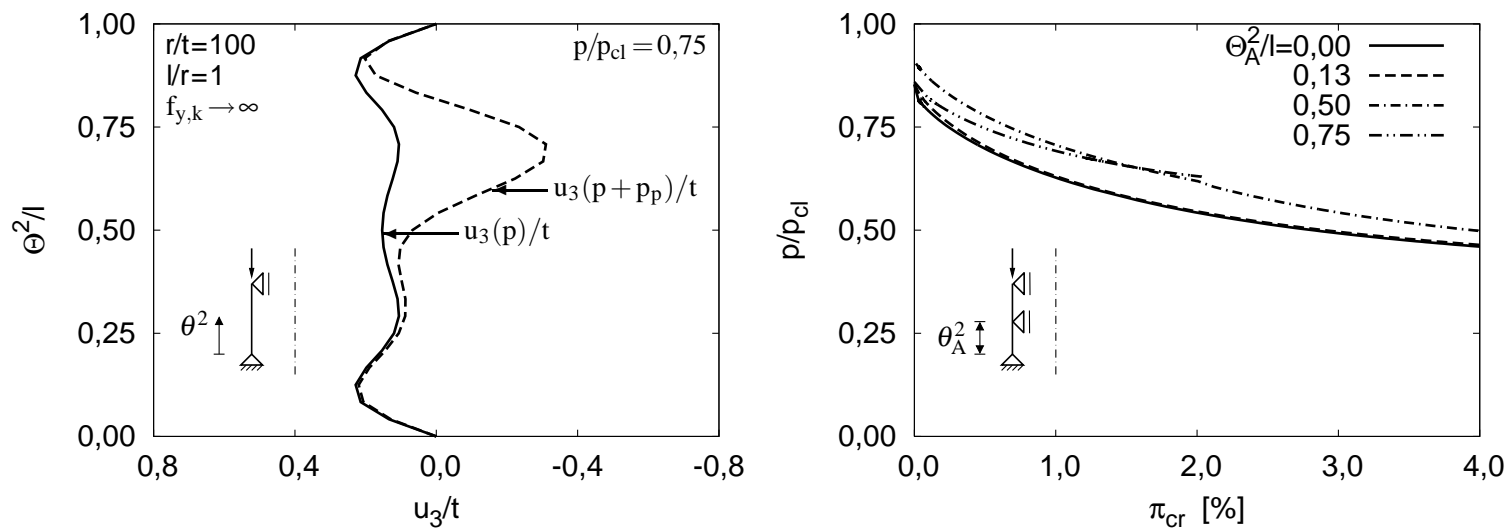

Bild 5.14: Einfluss ringförmiger Aussteifungen auf das Tragverhalten

Die Verformungen unterscheiden sich deutlich im oberen Bereich der Kreiszylinderschale. Daher könnten Aussteifungen in diesem Bereich die Imperfektionsempfindlichkeit merklich verringern. Für drei verschiedene Anordnungen der Aussteifung zeigt Bild 5.15 die Verformungen bei Erreichen des kritischen Zustandes.
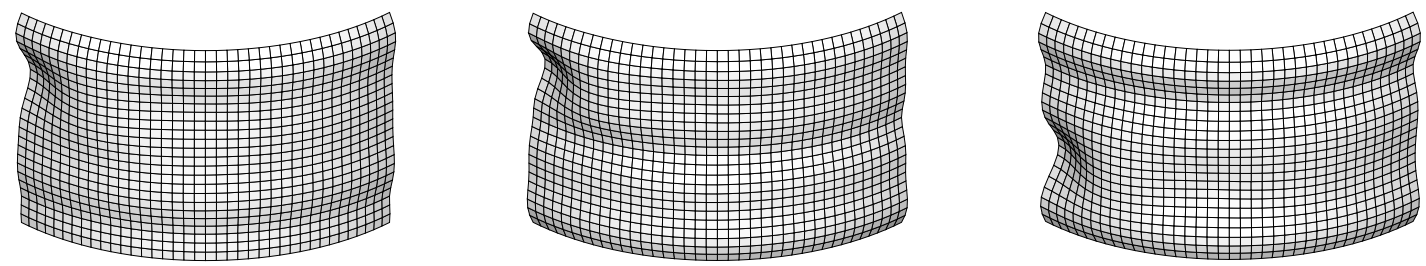

Bild 5.15: Verformungen im kritischen Zustand $\left(\theta_{A}^{2} / l=0,13,0,50\right.$ und 0,75) 
Für eine Aussteifung an der Position $\theta_{A}^{2} / l=0,13$ bzw. 0,50 sind die Verformungen bei Erreichen des kritischen Zustandes ähnlich denen der nicht ausgesteiften Kreiszylinderschale. Erfolgt die Aussteifung an der Position $\theta_{A}^{2} / l=0,75$, so sind die Verformungen durch eine lokale Einzelbeule im unteren Bereich der Kreiszylinderschale charakterisiert. Alle drei Aussteifungen verringern für ein definiertes Lastniveau die Imperfektionsempfindlichkeit. Die in Bild 5.14 angegebenen Verläufe der bezogenen Störenergie verdeutlichen aber, dass eine vom Lastniveau unabhängige Bewertung des Einflusses von Aussteifungen auf die Imperfektionsempfindlichkeit oftmals nicht möglich ist. Die Beullast ist für die Aussteifung im mittleren und das Nachbeulminimum für die Aussteifung im oberen Bereich am größten. Folglich sind mehrere Entwurfskriterien nicht unbedingt mit einer Aussteifung zu erfüllen.

\subsection{Kreiszylinderschalen aus Faserverbundwerkstoff}

Das Tragverhalten axialgedrückter Kreiszylinderschalen aus Faserverbundwerkstoff wird vom Aufbau des Faserverbundwerkstoffes beeinflusst. Zur Bewertung des Tragverhaltens mit dem Störenergie-Konzept ist die bisherige Normierung der Störenergie zu modifizieren. Dafür ist eine Bezugsgröße erforderlich, welche die Einheit der Störenergie besitzt und unabhängig von der Faserorientierung ist. Bei Faserverbundwerkstoffen, die aus UD-Schichten mit gleichen Materialparametern bestehen, ist mit

$$
B_{f w s}^{*}=\left(C_{B}^{1111}+C_{B}^{2222}+C_{B}^{1122}+C_{B}^{1212}+C_{B}^{1221}\right) f^{-1}
$$

eine entsprechende Bezugsgröße gegeben. Die physikalischen Komponenten $C_{\ddot{B}} \ldots$ sind aus den mit tensoriellen Feldgrößen formulierten konstitutiven Gleichungen (2.50) herleitbar und im orthogonalen Koordinatensystem $\theta^{\alpha}$ berechnet. Mit dem Parameter $f=2,5+0,5 \widetilde{v}_{12}$ ist die Summe aus Biegesteifigkeiten und Torsionssteifigkeit derart zu mitteln, dass im Grenzfall isotropen Materialverhaltens die Bezugsgröße $B_{f w s}^{*}$ identisch der effektiven Bezugsgröße $B^{*}$ nach Gl. (4.19) ist. Die Wirksamkeit der Normierung der Störenergie mit der Bezugsgröße $B_{f w s}^{*}$ wird in Bild 5.16 für am belasteten Rand wölbfrei gelagerte Kreiszylinderschalen gezeigt.

\begin{tabular}{|c|c|c|c|c|c|}
\hline $\mathrm{K} 1$ & ${ }_{i}[0 / 0 / 0 / 0]$ & $\mathrm{K} 3$ & {$[0 / 0 / 90 / 0]$} & $\mathrm{K} 5$ & {$[90 / 0 / 0 / 0]$} \\
\hline $\mathrm{K} 2$ & {$[0 / 0 / 0 / 90]$} & $\mathrm{K} 4$ & {$[0 / 90 / 0 / 0]$} & $\mathrm{K} 6$ & {$[90 / 90 / 90 / 90]$} \\
\hline \hline $\mathrm{W} 1$ & {$[0 / \mp \alpha / 0]$} & $\mathrm{W} 2$ & {$[-45 / \mp \alpha / 45]$} & $\mathrm{W} 3$ & {$[-90 / \mp \alpha / 90]$} \\
\hline
\end{tabular}

Tabelle 5.1: Faserverbundwerkstoffe ( $\theta^{1}$ ist der Umfangsrichtung zugeordnet)

Die Kreiszylinderschalen besitzen dieselbe Schlankheit und bestehen aus einem Faserverbundwerkstoff, dessen Fasergelege dem des Faserverbundwerkstoffes K2 nach Tabelle 5.1 entspricht. Die Materialparameter der UD-Schichten sind gegeben mit $\widetilde{E}_{1}=123.550 \mathrm{~N} / \mathrm{mm}^{2}, \widetilde{E}_{2}=8.708 \mathrm{~N} / \mathrm{mm}^{2}, \widetilde{v}_{12}=0,319$ und $\widetilde{G}_{12}=5.695 \mathrm{~N} / \mathrm{mm}^{2}[79]$. 

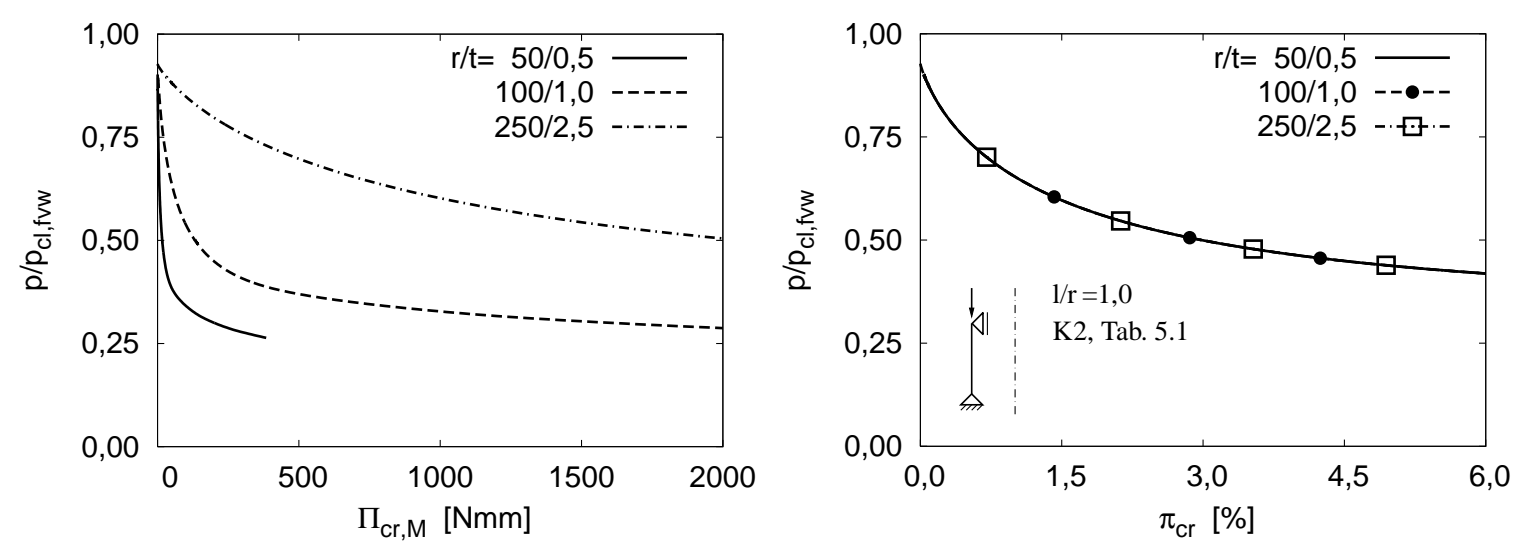

Bild 5.16: Störenergie und bezogene Störenergie vs. Lastniveau

Die klassische Beullast $p_{c l, f w s}$ folgt aus einer linearen Beulanalyse und ist wie die Bezugsgröße $B_{f w s}^{*}$ in Tabelle 5.2 aufgeführt. Wie nun Bild 5.16 verdeutlicht, beeinflus-

\begin{tabular}{|c|c|c|c|c|}
\hline$r / t$ & {$[\mathrm{~mm} / \mathrm{mm}]$} & $50 / 0,5$ & $100 / 1,0$ & $250 / 2,5$ \\
\hline \hline$p_{c l, f w s}$ & {$[\mathrm{~N} / \mathrm{mm}]$} & 72,7 & 145,3 & 363,3 \\
\hline$B_{f v w}^{*}$ & {$[\mathrm{Nmm}]$} & 555,0 & $4.440,3$ & $69.379,0$ \\
\hline
\end{tabular}

Tabelle 5.2: Beullast $p_{c l, f w s}$ und Bezugsgröße $B_{f v w}^{*}$

sen Radius und Wanddicke für Kreiszylinderschalen mit gleicher Schlankheit und aus Faserverbundwerkstoffen, die gleiche Fasergelege und deren UD-Schichten gleiche Materialparameter aufweisen, signifikant die Störenergie. Dahingegen ermöglicht die Normierung der Störenergie mit der Bezugsgröße $B_{f w s}^{*}$ eine adäquate Bewertung der Imperfektionsempfindlichkeit der verschiedenen Kreiszylinderschalen.

Die Bewertung des Tragverhaltens axialgedrückter Kreiszylinderschalen erfolgt für die in Tabelle 5.1 angegebenen Faserverbundwerkstoffe. Die Faserverbundwerkstoffe dienen der Untersuchung stabilitätstheoretischer Aspekte und berücksichtigen weniger etwaige konstruktive Gesichtspunkte. Die Faserverbundwerkstoffe bestehen aus vier 0,125 mm dicken UD-Schichten, deren Materialparameter den oben erwähnten entsprechen. Die Orientierung der Einzelschichten ist vom Faserverbundwerkstoff abhängig. Im Gegensatz zu Kreiszylinderschalen aus den Faserverbundwerkstoffen K1 und K6 treten bei den unsymmetrischen Kreuzverbunden K2 bis K5 zusätzliche Randstörungen infolge der Kopplung von Membran- und Biegetragwirkung auf. So bewirkt die Anordnung der $90^{\circ}$-Schicht weiter nach außen eine Verringerung der von der Querdehnzahl abhängigen Randstörungen infolge Grundbelastung. Für Kreiszylinderschalen aus den Faserverbundwerkstoffen K1-K6 zeigt Bild 5.17 den Einfluss der Orientierung der Einzelschichten auf Beullast und Imperfektionsempfindlichkeit.

Für Kreiszylinderschalen aus den Faserverbundwerkstoffen K1 und K6 lässt der Verlauf der bezogenen Störenergie die Zunahme der Beullast und der Imperfektionsemp- 

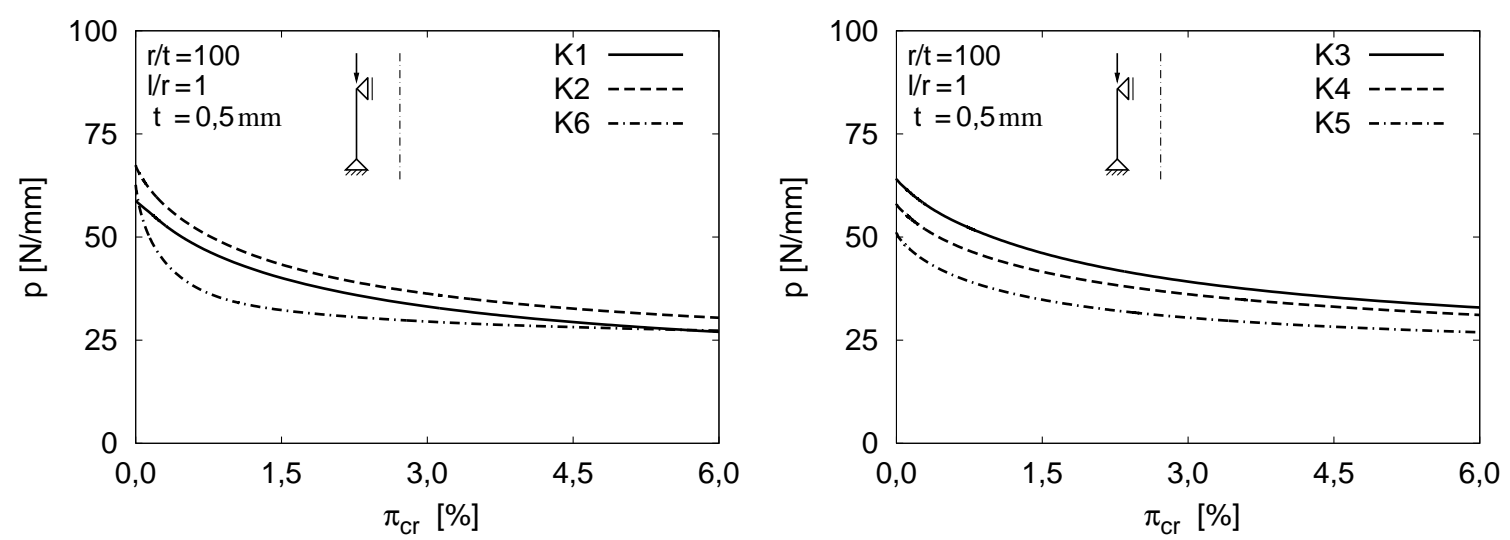

Bild 5.17: Bezogene Störenergie vs. Faserverbundwerkstoff und Lastniveau

findlichkeit bei ansteigender axialer Steifigkeit erkennen. Für die Kreuzverbunde K3 bis K5 nimmt mit fortschreitender Anordnung der $90^{\circ}$-Schicht nach innen die Beullast ab und die Imperfektionsempfindlichkeit für ein definiertes Lastniveau zu. Letzteres trifft indes nicht für Kreiszylinderschalen aus Faserverbundwerkstoff K2 zu.

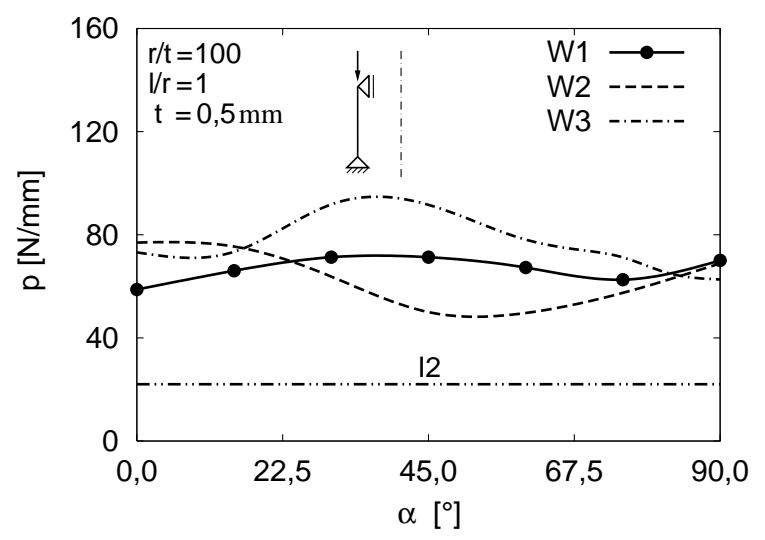

Bild 5.18: Beullast vs. Faserverbundwerkstoff

Für Kreiszylinderschalen aus Winkelverbunden sind Beullast und Imperfektionsempfindlichkeit signifikant vom Faserwinkel $\alpha$ abhängig. So ist die Differenz zwischen den in Bild 5.18 angegebenen Beullasten für Faserverbundwerkstoff W3 mit 29,1 N/mm maximal und für Faserverbundwerkstoff $\mathrm{W} 1 \mathrm{mit}$ 12,5 N/mm minimal. Die maximale Differenz zwischen allen angegebenen Beullasten beträgt 42,1 N/mm. Zur Verdeutlichung der beullasterhöhenden Wirkung der Fasern und der Notwendigkeit des Entwurfes optimaler Fasergelege ist die Beullast einer Kreiszylinderschale I2 aufgeführt, deren isotropes Materialverhalten mit $E=\widetilde{E}_{2}$ und $v=\widetilde{v}_{12}$ beschrieben ist.

Die in Bild 5.19 dargestellten Beuleigenformen sind ähnlich zu Beuleigenformen von Kreiszylinderschalen mit isotropem Materialverhalten. Für Faserverbundwerkstoff W3/00 entspricht die Beuleigenform nahezu einem mit der Beultheorie nach Flügge zu ermittelnden Beulmuster axialgedrückter Kreiszylinderschalen [124]. Für Faserverbundwerkstoff W1/15 bzw. W3/60 zeigt die Beuleigenform Ähnlichkeit zur Beuleigenform von Kreiszylinderschalen unter Torsion bzw. Radialaußendruck [144]. 


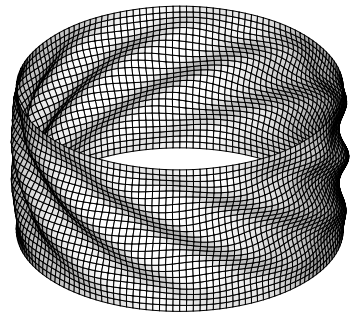

$\mathrm{W} 1 / 15$

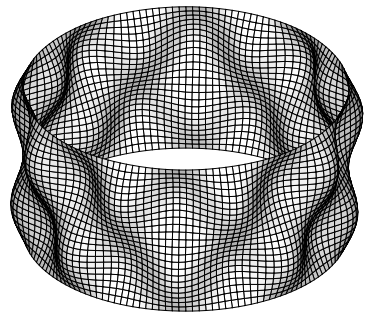

$\mathrm{W} 3 / \alpha=0^{\circ}$

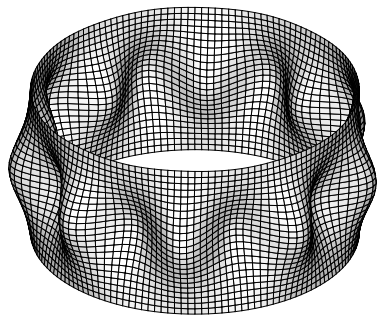

$\mathrm{W} 3 / 60$

Bild 5.19: Beuleigenform vs. Faserverbundwerkstoff

Allgemein können die Beuleigenformen von Kreiszylinderschalen aus Faserverbundwerkstoff auch Kombinationen der dargestellten Beuleigenformen repräsentieren. Störlast und zugehörige Verformungen sind ebenfalls vom Faserwinkel abhängig. Die Störlast umfasst Kraftgrößen und Verzerrungen. In Bild 5.20 sind für zwei Kreiszylinderschalen und jeweils ein Lastniveau die wesentlichen Kraftkomponenten der in diskretisierter Form vorliegenden Störlast dargestellt. Diese sind im Vergleich zur Grundbelastung klein. So beträgt die maximale radial gerichtete Kraftkomponente der Störlast für die Kreiszylinderschale aus Faserverbundwerkstoff W3/60 ca. 0,1 N.

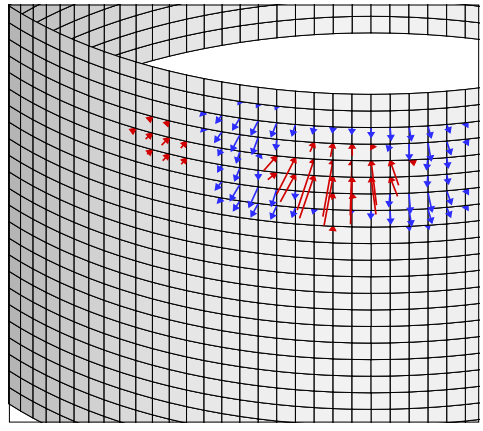

$\mathrm{W} 1 / 15, p=55,8 \mathrm{~N} / \mathrm{mm}$

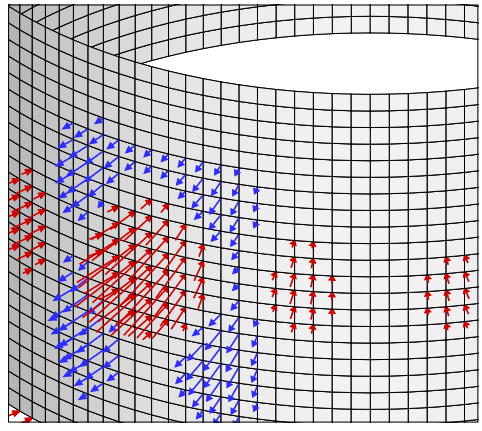

$\mathrm{W} 3 / 60, p=73,3 \mathrm{~N} / \mathrm{mm}$

Bild 5.20: Wesentliche Kraftkomponenten der Störlast

Die Wirkung der Störlasten auf die Verformungen der jeweiligen Kreiszylinderschale verdeutlicht Bild 5.21. Für die Faserverbundwerkstoffe W3/00 und W3/60 sind die Verformungen infolge Störlast lokal sehr ähnlich der Beuleigenform. Für Faserverbundwerkstoff W1/15 weisen beide Verformungsmoden nur geringe Ähnlichkeit auf.

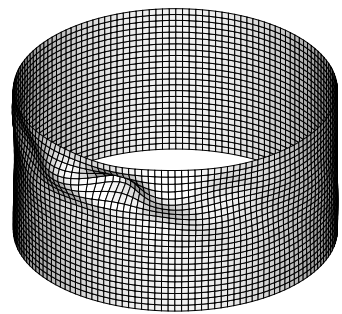

$\mathrm{W} 1 / 15$

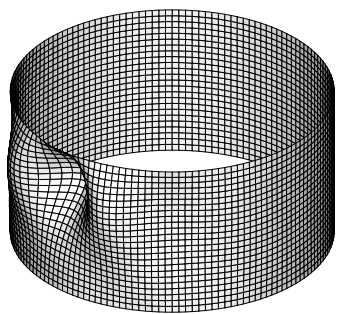

$\mathrm{W} 3 / 00$

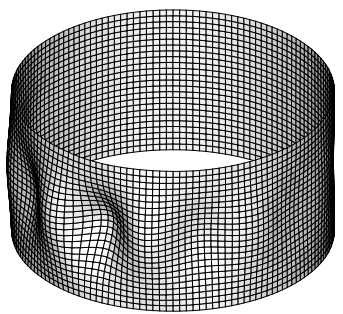

$\mathrm{W} 3 / 60$

Bild 5.21: Verformungen infolge Störlast vs. Faserverbundwerkstoff

Die Verformungen infolge Störlast sind für viele Faserverbundwerkstoffe durch eine lokale Einzelbeule charakterisiert. Der Abstand der Einzelbeule vom belasteten Rand, 
deren Form, mehr oder wenig ellipsenförmig, und Orientierung sind vom Faserwinkel und somit von der Steifigkeitsverteilung abhängig. Den Einfluss verschiedener Faserverbundwerkstoffe auf die Imperfektionsempfindlichkeit stellt Bild 5.22 heraus.
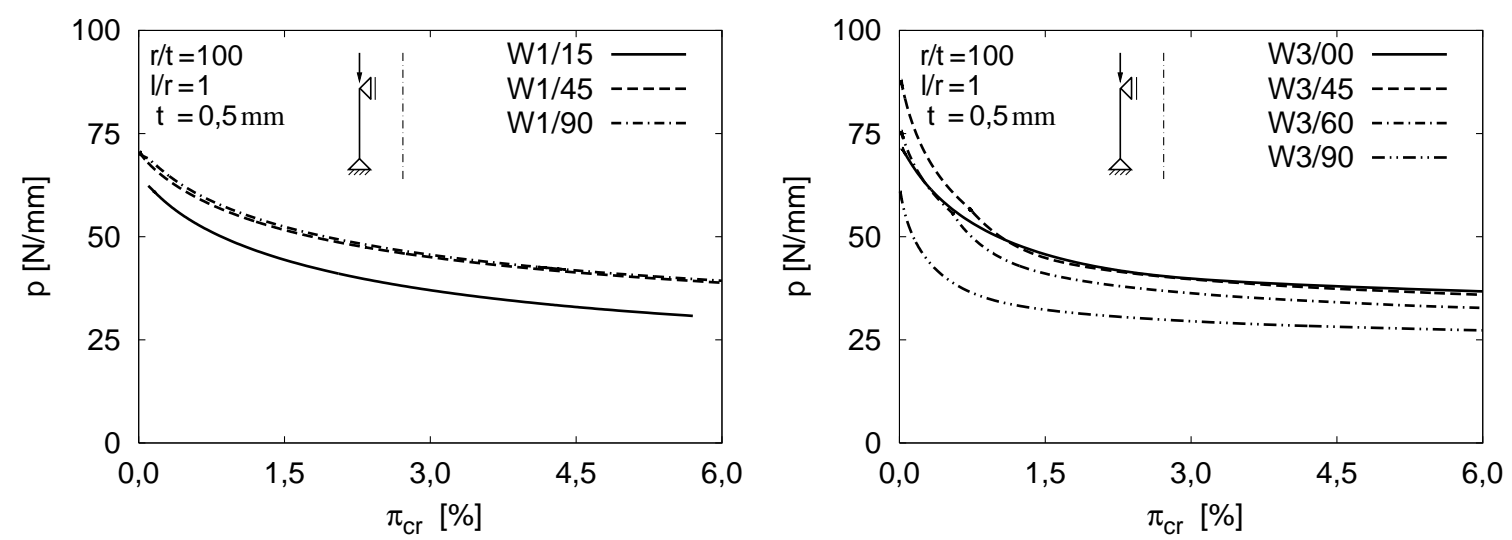

Bild 5.22: Imperfektionsempfindlichkeit vs. Faserverbundwerkstoff und Lastniveau

Für die Faserverbundwerkstoffe vom Typ W1 ist die Steigung des Verlaufes der bezogenen Störenergie für höhere Lastniveaus geringer und für niedrige Lastniveaus etwas größer als für die Faserverbundwerkstoffe vom Typ W3. Grund hierfür ist insbesondere die unterschiedliche Orientierung der äußeren Schichten. Allgemein sind der Verlauf der bezogenen Störenergie und somit die Imperfektionsempfindlichkeit sowie die Differenz zwischen zwei Verläufen deutlich vom Faserwinkel abhängig.

Für eine Kreiszylinderschale aus Faserverbundwerkstoff $\mathrm{W} 7_{i}[\mp \alpha, \mp \beta]$, deren weitere Systemparameter denen in den vorherigen Untersuchungen entsprechen, ist die Abhängigkeit der Beul- und der Grenzlast von den Faserwinkeln in Bild 5.23 aufgezeigt.
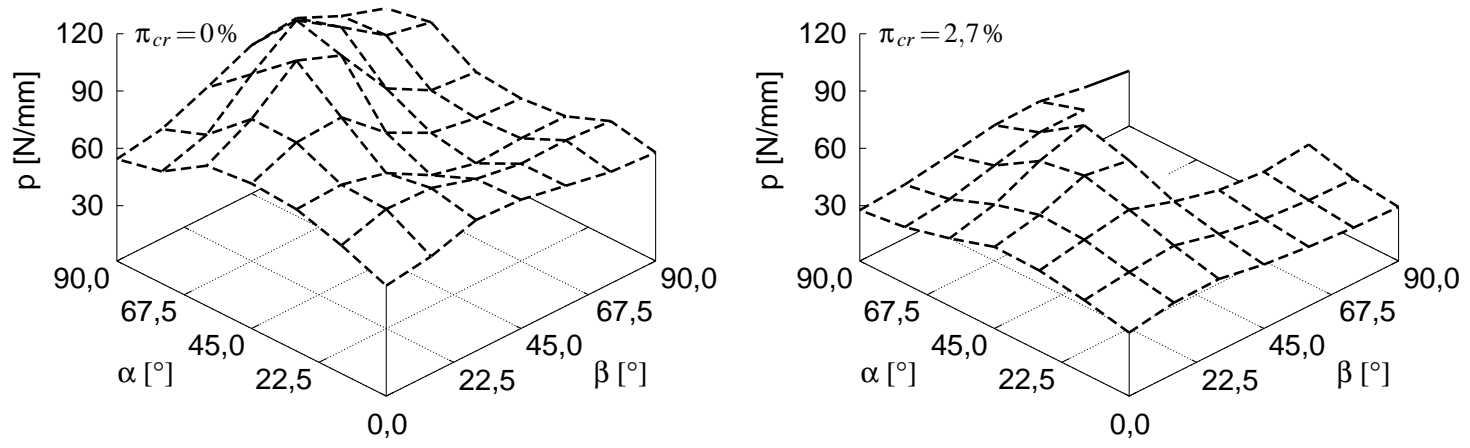

Bild 5.23: Beullast und Grenzlast vs. Faserwinkel $\alpha$ und $\beta$

Die Beullast besitzt für mehrere Konstellationen der Faserwinkel ein lokales Extremum. Die minimale Beullast tritt mit $50,7 \mathrm{~N} / \mathrm{mm}$ bei $\alpha=45^{\circ}, \beta=90^{\circ}$ und die maximale Beullast mit $105,9 \mathrm{~N} / \mathrm{mm}$ bei $\alpha=60^{\circ}, \beta=30^{\circ}$ auf. Für $\alpha=15^{\circ}$ wird die Beullast nur geringfügig vom Faserwinkel $\beta$ beeinflusst. Die Differenz zwischen Beul- und Grenzlast variiert aufgrund der vom Faserverbundwerkstoff abhängigen Imperfektionsempfindlichkeit, vgl. Bild 5.22. So ist die Grenzlast für $\alpha=90^{\circ}, \beta=0^{\circ}$ mit $27,6 \mathrm{~N} / \mathrm{mm}$ minimal und für $\alpha=60^{\circ}, \beta=45^{\circ} \mathrm{mit} 60,3 \mathrm{~N} / \mathrm{mm}$ maximal. Für meh- 
rere Fälle ist die Grenzlast nicht angegeben, da der zum Referenzwert der bezogenen Störenergie korrespondierende kritische Zustand nicht existiert oder dessen Identifikation misslingt. Die angegebenen Grenzlasten sind indes noch anhand einschlägiger Regelwerke und experimenteller Untersuchungen zu verifizieren [109, 79].

Das Tragverhalten axialgedrückter Kreiszylinderschalen aus Faserverbundwerkstoff ist auch von der Anzahl $n_{l}$ der Einzelschichten abhängig. Diesen Aspekt verdeutlicht Bild 5.24 links für symmetrische Faserverbundwerkstoffe K7 $[(90,0), \cdots,(90,0)]_{s}$.
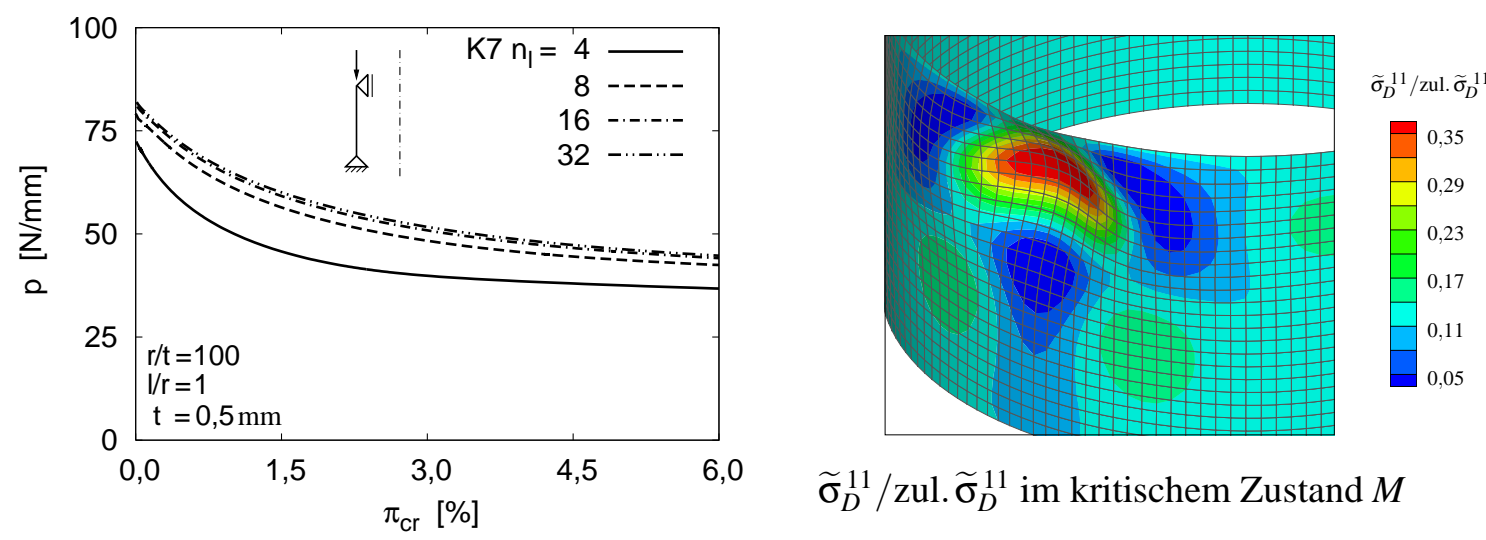

$\widetilde{\sigma}_{D}^{11} /$ zul. $\widetilde{\sigma}_{D}^{11}$ im kritischem Zustand $M$

Bild 5.24: Einfluss der Anzahl der Einzelschichten sowie Spannungsverteilung

Mit ansteigender Anzahl der Einzelschichten nehmen Beul- und Grenzlast zu sowie die Imperfektionsempfindlichkeit für ein definiertes Lastniveau ab. Beuleigenform und Verformungen infolge Störlast verändern sich jedoch nur unwesentlich. Für letztere tritt die maximale Verformung mit ansteigender Anzahl der Einzelschichten näher am belasteten Rand auf. Ursache der Abhängigkeit des Tragverhaltens von der Anzahl der Einzelschichten ist primär das Verhältnis zwischen den Biegesteifigkeiten $C_{B}^{1111}$ und $C_{B}^{2222}$. Das Verhältnis beträgt bei vier Einzelschichten 0,21 und bei 32 Einzelschichten 0,85. Somit erscheint für axialgedrückte Kreiszylinderschalen die Verwendung von Faserverbundwerkstoffen, die in Meridian- und Umfangsrichtung eine annähernd gleich große Biegesteifigkeit aufweisen, erstrebenswert.

Die Bewertung des Spannungszustandes erfolgt exemplarisch für den Faserverbundwerkstoff K7, der aus 32 Schichten besteht. Bild 5.24 rechts zeigt für den kritischen Zustand, der dem Grundzustand auf dem Lastniveau $p=52,8 \mathrm{~N} / \mathrm{mm}$ zugehörig ist, die geringe Ausnutzung der zulässigen Spannung $\widetilde{\sigma}_{D}^{11}=-1.605 \mathrm{~N} / \mathrm{mm}^{2}$ an der maßgebenden äußeren Schalenwandung auf. Eine ähnlich geringe Ausnutzung der zulässigen Spannung lässt sich auch für die anderen Spannungen im Faserverbundwerkstoff zeigen. Somit ist vor Erreichen des der statischen Grenzlast zugehörigen kritischen Zustandes ein Versagen des Faserverbundwerkstoffes nicht zu erwarten. 


\section{Statische Grenzlasten von Kugelschalen}

Die Imperfektionsempfindlichkeit und die Grenzlasten von $\mathrm{Ku}-$ gelschalen sind von der Belastung und Geometrie sowie von den Randbedingungen und Materialparametern abhängig. Dabei ist die Imperfektionsempfindlichkeit trotz des zweidimensionalen Membranspannungszustandes in ausgewählten Fällen ähnlich der axialgedrückter Kreiszylinderschalen.

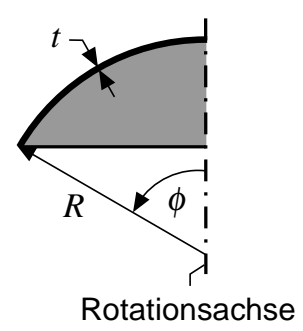

\subsection{Konstante Radialdruckbelastung}

Die klassische Beullast einer Vollkugelschale unter konstantem Radialdruck wird von Zoelly [169] für linear elastisches, isotropes Materialverhalten zu

$$
p_{c l}=\frac{2}{\sqrt{3\left(1-v^{2}\right)}} E\left(\frac{t}{R}\right)^{2}
$$

ermittelt. Die Beullast gilt für rotationssymmetrisches und nicht rotationssymmetrisches Beulen [147] und wird nachfolgend zur Beschreibung der Grenzlasten von $\mathrm{Ku}$ gelschalen mit elastischem Materialverhalten verwendet. Der Parameter

$$
\lambda=\sqrt[4]{12\left(1-v^{2}\right)} \sqrt{\frac{R}{t}} \phi
$$

wird in der Literatur oft als Bezugsgröße für die Grenzlasten herangezogen, impliziert aber identische Grenzlasten für ausgewählte Konstellationen der geometrischen Parameter und ist somit kein objektives Maß für die Grenzlasten [153, 2]. In LastVerformungs-Diagrammen wird das Lastniveau über der gemittelten Durchbiegung

$$
\bar{u}_{3}=\frac{1}{A} \int_{A} u_{3} d A
$$

aufgetragen. Mit dieser Durchbiegung ist das Verformungsverhalten bei lokalen Beulphänomenen aber oftmals nicht hinreichend genau beschreibbar. Die Imperfektionsempfindlichkeit von Kugelschalen wird u.a. von dem Radius, der Wanddicke und dem meridionalen Öffnungswinkel $\phi$ beeinflusst. Mit ansteigendem Radius nimmt bei konstanter Schlankheit $R / t$ die Störenergie für ein auf die klassische Beullast 
bezogenes Lastniveau zu, die bezogene Störenergie bleibt jedoch unverändert, vgl. Bild 5.16. Somit sind die Schlankheit und der meridionale Öffnungswinkel wesentliche geometrische Parameter, deren Einfluss auf die Imperfektionsempfindlichkeit und die statische Grenzlast nachfolgend mit dem Störenergie-Konzept analysiert wird.

Dem Vergleich der ermittelten Grenzlasten mit denen der Regelwerke liegen folgende Voraussetzungen zugrunde: Der im Abminderungsfaktor $\alpha$ nach DASt-Richtlinie 013 enthaltene zusätzliche Abminderungsfaktor 0,75 wird bei der Ermittlung der zur Beulspannung gehörenden Belastung nicht berücksichtigt. Gleiches gilt für den Teilsicherheitsbeiwert $\gamma=4 / 3$, der in den Regelungen der ECCS-Recommendations enthalten ist. Außerdem ist zwischen dem bezogenen Schlankheitsgrad nach DIN 18800 und dem nach den ECCS-Recommendations die Relation

$$
\bar{\lambda}_{S}=\frac{\bar{\lambda}_{E C C S}}{\sqrt{C_{K}}},
$$

zu beachten, wobei $C_{K}=1,0$ gesetzt wird, da in den ECCS-Recommendations Grenzlasten von Vollkugelschalen unter Radialdruck angegeben sind [2]. Zwischen dem Schlankheitsgrad nach DIN 18800 und dem nach Draft 2000 [2] wird die Relation

$$
\bar{\lambda}_{S}=\bar{\lambda}_{\text {Draft }} \sqrt{\frac{C_{c}}{C_{K} C_{p l}}}
$$

berücksichtigt. Die Faktoren $C_{c}, C_{K}$ und $C_{p l}$ dienen der Erfassung des Einflusses der Randbedingungen und des Materialverhaltens und können wie die Definition der Schlankheitsgrade den Regelwerken bzw. der Draft 2000 entnommen werden.

\subsubsection{Eingespannte Kugelschalen}

Auch für diesen Beulfall beeinflussen die mit den Randbedingungen einhergehenden Störungen des Membranspannungszustandes signifikant das Tragverhalten. Dies verdeutlichen die für Kugelschalen der Schlankheit $R / t=100$ und ein definiertes Lastniveau in Bild 6.1 zehnfach überhöht dargestellten Verformungen infolge Störlast (4.7).

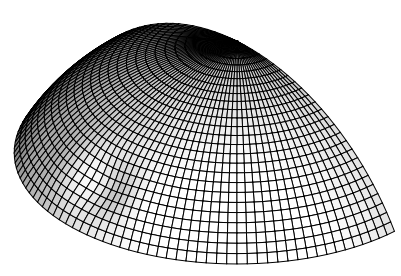

$\phi=60^{\circ}$

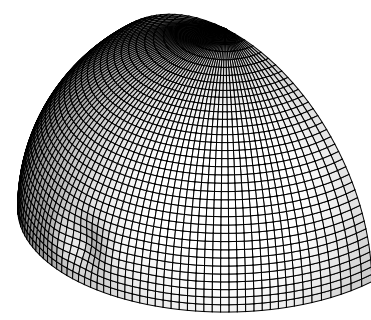

$\phi=90^{\circ}$

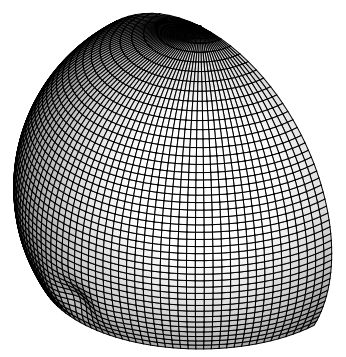

$\phi=120^{\circ}$

Bild 6.1: Verformungen infolge Störlast vs. meridionaler Öffnungswinkel $\left(p / p_{c l}=0,7\right)$ 
Die Verformungen sind unabhängig vom meridionalen Öffnungswinkel durch eine lokale Einzelbeule im Bereich des eingespannten Randes gekennzeichnet. Die Amplitude der Verformungen beträgt annähernd ein Drittel der Wanddicke. Die Einzelbeule klingt in Breitenkreis- und Meridianrichtung zwar schnell ab, zerstört aber den für den Lastabtrag im Grundzustand charakteristischen rotationssymmetrischen Spannungs- bzw. Verformungszustand. Die Verformungen infolge Störlast sind, wie in Bild 6.2 überhöht aufgezeigt, vom Lastniveau des Grundzustandes abhängig.

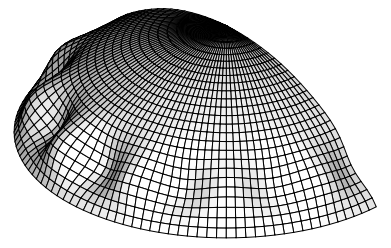

$p / p_{c l}=0,78$

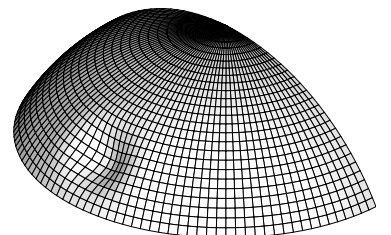

$p / p_{c l}=0,50$

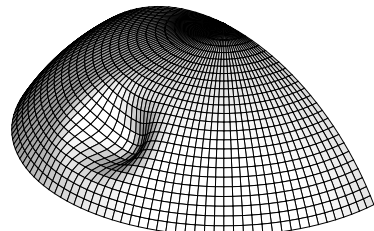

$p / p_{c l}=0,30$

Bild 6.2: Verformungen infolge Störlast vs. Lastniveau (Überhöhungsfaktoren 100, 10, 10)

Im Bereich des ersten Verzweigungspunktes im primären Last-Verformungs-Pfad sind die Verformungen infolge Störlast ähnlich der ersten Beuleigenform der Kugelschale. Für niedrigere Lastniveaus sind die Verformungen durch die lokale Einzelbeule charakterisiert. Die Einzelbeule ist mit abnehmendem Lastniveau weiter vom eingespannten Rand entfernt lokalisiert, da sich die Verformungen infolge Störlast denen im Nachbeulminimum annähern. Die Amplitude der Verformungen wächst für $p / p_{c l}=0,30$ auf fast den doppelten Wert der Wanddicke an und verdeutlicht, dass die Störenergie im Wesentlichen aus lokal konzentrierter Biegeenergie besteht und die Normierung (4.20) der Störenergie gerechtfertigt ist.
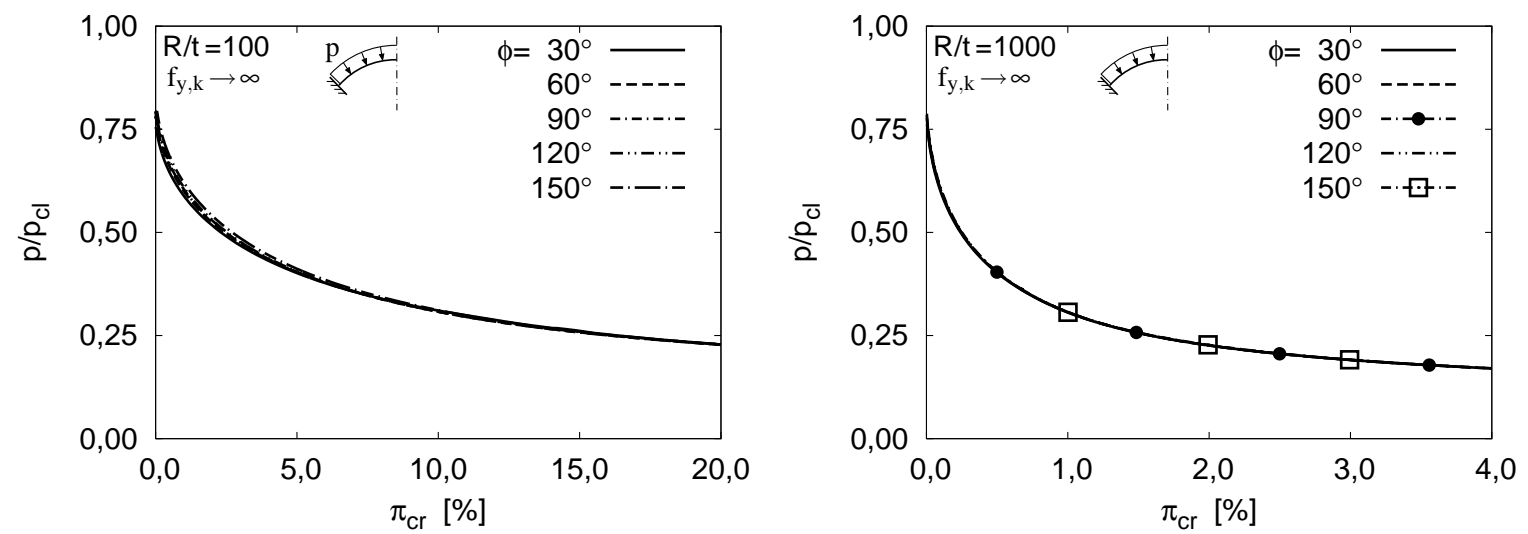

Bild 6.3: Bezogene Störenergie vs. meridionaler Öffnungswinkel

Den geringen Einfluss des Öffnungswinkels auf die große Imperfektionsempfindlichkeit eingespannter Kugelschalen bestätigen die in Bild 6.3 angegebenen Verläufe der bezogenen Störenergie. Für gedrungene Kugelschalen ist die Imperfektionsempfindlichkeit bei hohen Lastniveaus aber noch merklich vom Öffnungswinkel abhängig. Dabei nimmt die bezogene Störenergie für ein definiertes Lastniveau mit ansteigendem Öffnungswinkel zu. Für schlanke Kugelschalen ist der Einfluss des Öffnungswinkels 
jedoch sehr gering. Demzufolge ist der Gültigkeitsbereich der Grenzlasten nach DIN 18800 und Draft 2000 mit $\phi \leq 135^{\circ}$ etwas zu konservativ festgelegt.
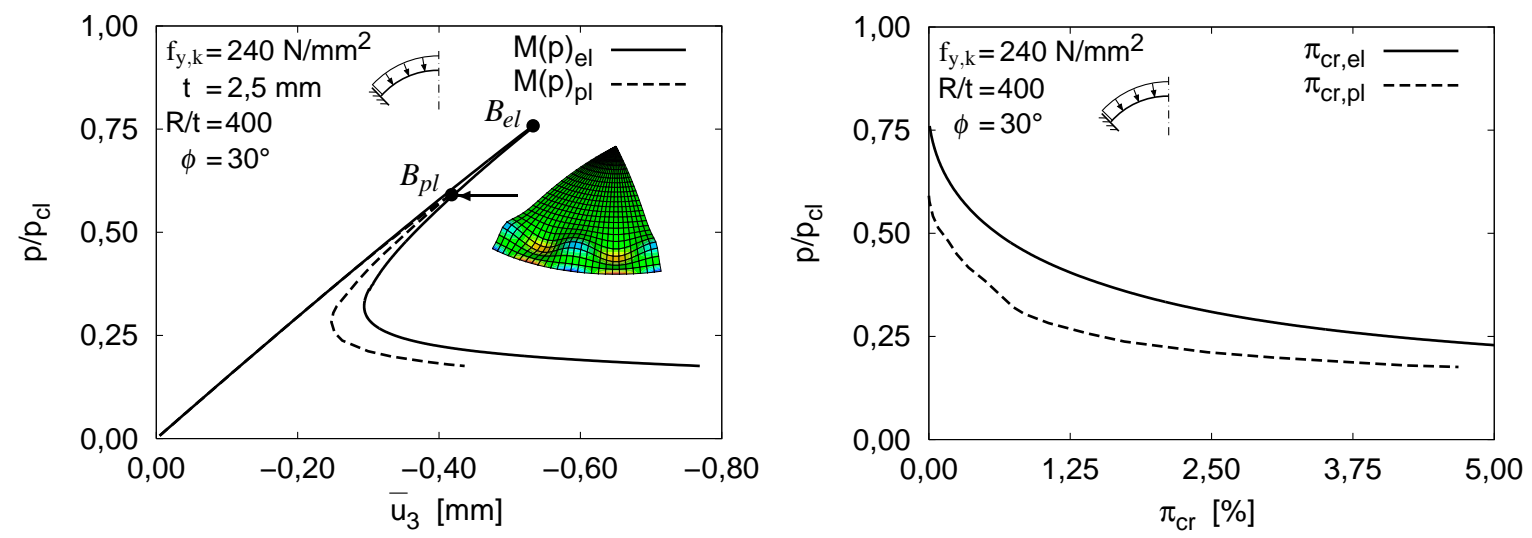

Bild 6.4: Tragverhalten vs. Plastifizierung des Schalenkontinuums

Der Einfluss der Plastifizierung des Schalenkontinuums auf das Tragverhalten wird nachfolgend an Kugelschalen mit dem Öffnungswinkel $\phi=30^{\circ}$ und den Materialparametern nach Abschnitt5.1 bewertet. Für eine Kugelschale der Schlankheit $R / t=$ 400 plastifiziert das Schalenkontinuum erst für höhere Lastniveaus. Beullast und Anzahl der Beulwellen in Breitenkreisrichtung verringern sich, wie Bild 6.4 aufzeigt, aufgrund der mit der Plastifizierung einhergehenden Abnahme der Steifigkeiten. Die gleichzeitig stattfindende Verringerung des Abstandes zwischen Grundzustand und kritischem Zustand $M$ wird auch durch die Änderung der bezogenen Störenergie verdeutlicht. Ferner repräsentiert der Referenzwert der bezogenen Störenergie annähernd das Nachbeulminimum; eine Charakteristik, die sich bei elastisch-plastischem Materialverhalten mit abnehmender Schlankheit verstärkt. Die von der Störlast initiierte Plastifizierung des Schalenkontinuums beginnt gemäß Bild 6.5 im Bereich der größten Lastordinate und erfolgt nahezu gleichmäßig in Breitenkreis- und Meridianrichtung, aber auch in Richtung der Schalennormalen.
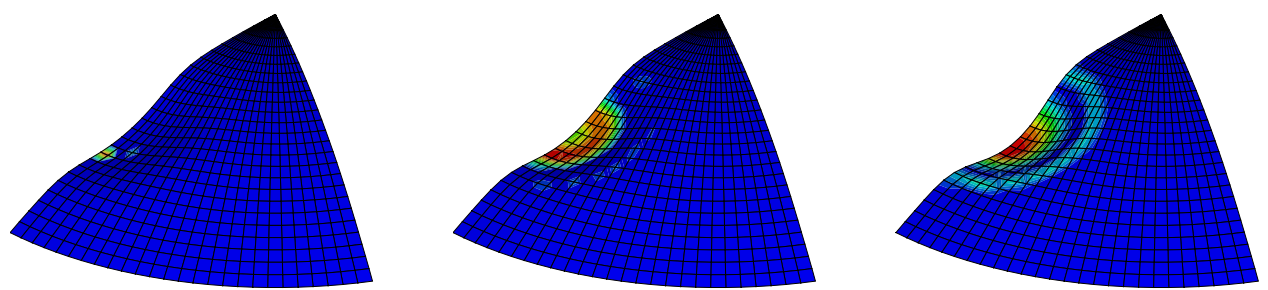

Bild 6.5: Plastifizierung des Schalenkontinuums infolge Störlast $\left(p / p_{c l}=0,33\right)$

Für elastisches Materialverhalten verdeutlicht Bild 6.6 links den Einfluss der bezogenen Störenergie und der Schlankheit auf die Grenzlasten eingespannter Kugelschalen. Die Differenz zwischen den mittels der angegebenen bezogenen Störenergien definierbaren Grenzlasten nimmt bei konstanter Schlankheit mit ansteigender bezogener Störenergie ab und ist für mittelschlanke Kugelschalen maximal. Ein Vergleich mit Bild 5.3 zeigt, dass die DASt-Richtlinie 013 die Grenzlasten eingespannter $\mathrm{Ku}$ - 
gelschalen unter Radialdruck und axialgedrückter Kreiszylinderschalen nicht mit einem energetisch äquivalenten Einfluss von Störungen beschreibt.
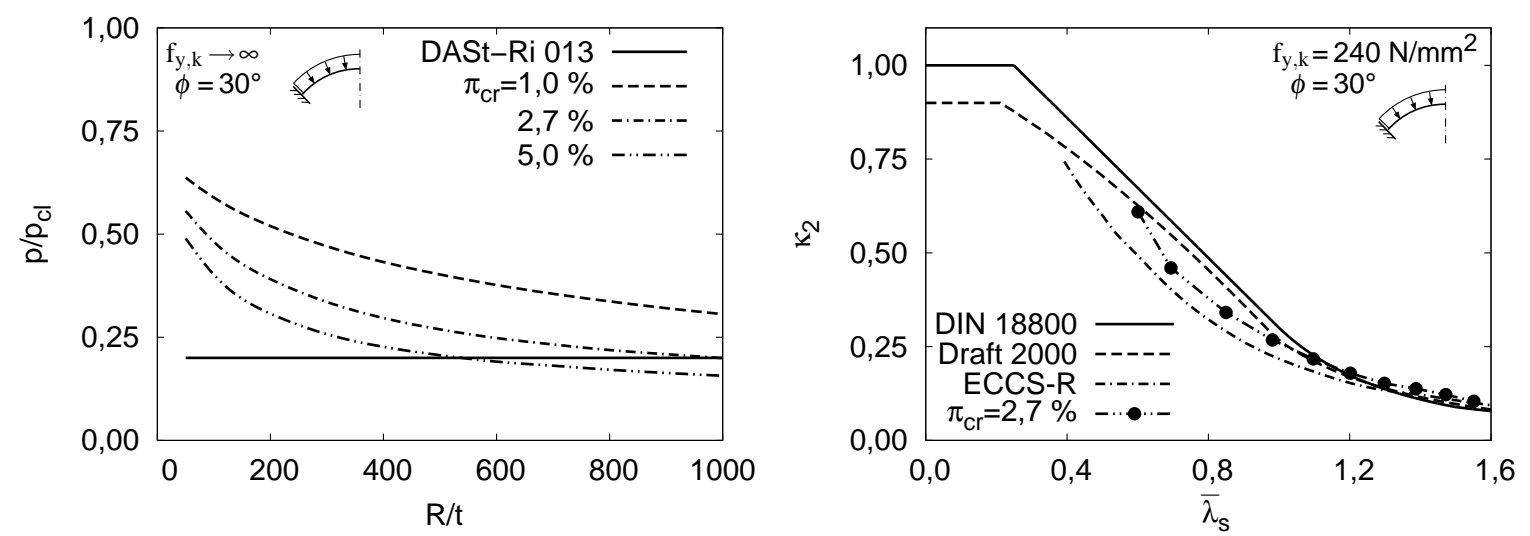

Bild 6.6: Grenzlasten eingespannter Kugelschalen

In Bild 6.6 rechts sind für den vorliegenden Beulfall die mit dem Referenzwert der bezogenen Störenergie definierten Grenzlasten denen nach DIN 18800 und den ECCSRecommendations gegenübergestellt. Ferner sind die Grenzlasten nach Draft 2000 angegeben. Für mittelgroße bezogene Schlankheitsgrade $\bar{\lambda}_{s}$ liegen die Grenzlasten gemäß Referenzwert der bezogenen Störenergie zwischen denen der Regelwerke, zeigen tendenziell aber bessere Übereinstimmung mit den Grenzlasten nach den ECCSRecommendations. Für große Schlankheitsgrade erfolgt die Beschreibung der Grenzlasten mit nahezu gleichen Abminderungsfaktoren. Die Grenzlasten nach DIN 18800 und Draft 2000 unterscheiden sich nur bei kleinen Schlankheitsgraden deutlich.

\subsubsection{Unverschieblich gelenkig gelagerte Kugelschalen}

Im Vergleich zu eingespannten Kugelschalen besitzen unverschieblich gelenkig gelagerte Kugelschalen eine weichere Lagerung, mit der eine Verringerung der Beullast einhergeht. Für konstanten Radialdruck differieren die Beuleigenformen und die Verformungen infolge Störlast nur geringfügig von denen eingespannter Kugelschalen. Ähnliches gilt, wie nachfolgend gezeigt wird, für die Imperfektionsempfindlichkeit.

Für die in Bild 6.7 angegebenen Schlankheiten beeinflusst der meridionale Öffnungswinkel die Steigung des Verlaufes der bezogenen Störenergie nur unwesentlich. Dabei nimmt die bezogene Störenergie für ein definiertes Lastniveau bei ansteigendem Öffnungswinkel zu. Unterschiede in der bezogenen Störenergie sind für kleine Schlankheiten aber noch im Bereich des Nachbeulminimums erkennbar.

Die räumliche Verteilung der bezogenen Störenergie ist von den Verformungen infolge Störlast und somit auch vom Lastniveau abhängig. Für eine Kugelschale mit der Schlankheit $R / t=100$ und dem Öffnungswinkel $\phi=30^{\circ}$ sowie für zwei Lastniveaus 

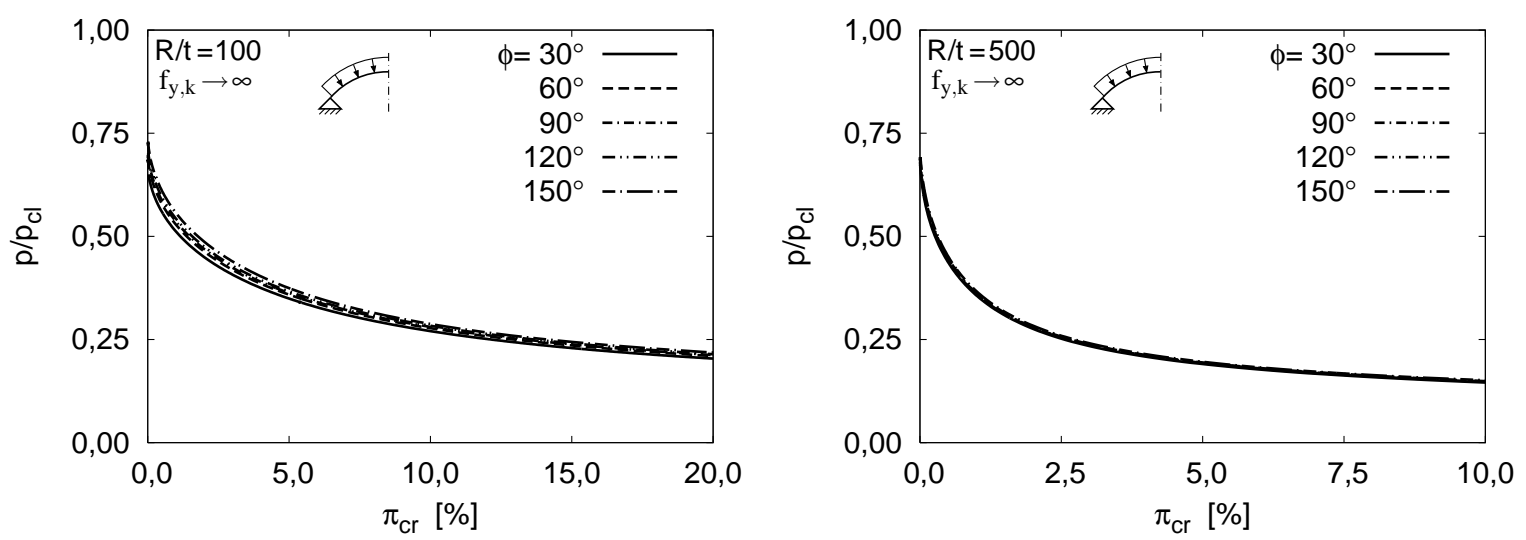

Bild 6.7: Bezogene Störenergie vs. meridionaler Öffnungswinkel

ist in Bild 6.8 neben den fünfzehnfach überhöhten Verformungen infolge Störlast die räumliche Verteilung der bezogenen Störenergie elementweise dargestellt.

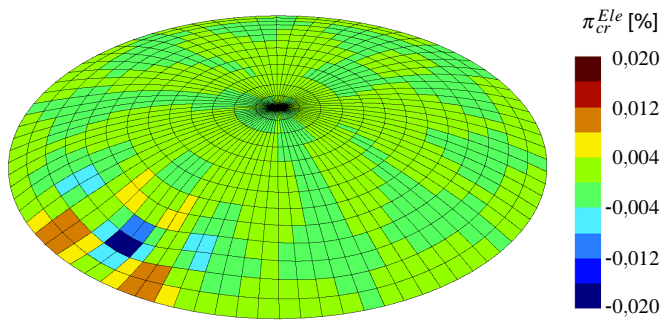

$p / p_{c l}=0,62, \pi_{c r}=0,1 \%$

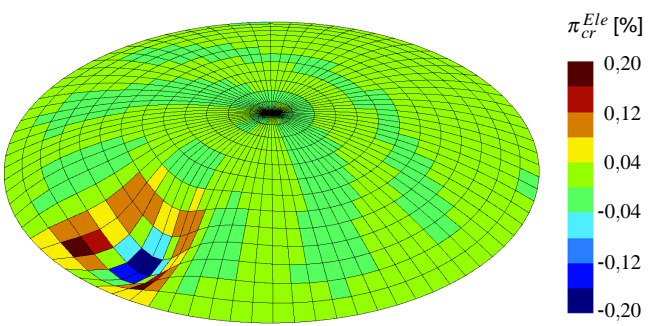

$p / p_{c l}=0,42, \pi_{c r}=2,6 \%$

Bild 6.8: Verteilung der bezogenen Störenergie vs. Lastniveau

Signifikante positive Anteile der bezogenen Störenergie sind für das Lastniveau $p / p_{c l}$ $=0,62$ am gelagerten Rand und für das Lastniveau $p / p_{c l}=0,42$ in geringem Abstand um den Bereich der Amplitude der Verformungen infolge Störlast erkennbar. Dagegen ist im Bereich der Amplitude der Verformungen die bezogene Störenergie unabhängig vom Lastniveau negativ, da hier primär die von den Zustandsgrößen kubisch abhängigen Terme des inkrementellen Potentials das Tragverhalten kennzeichnen.
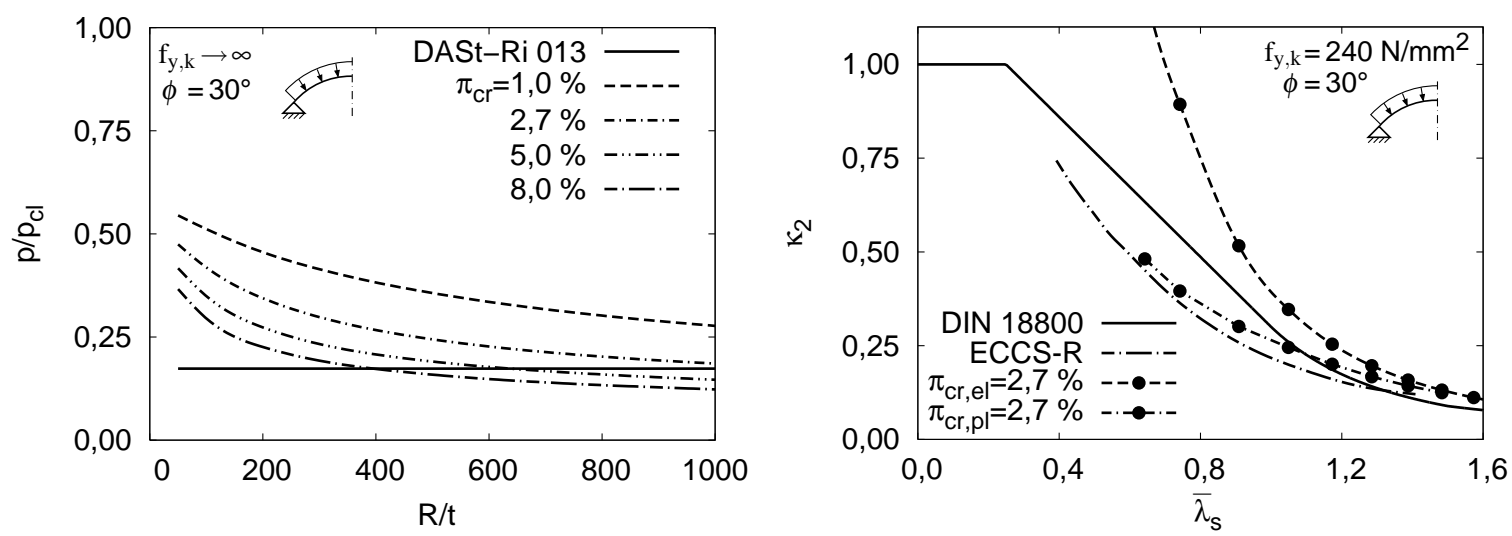

Bild 6.9: Grenzlasten unverschieblich gelenkig gelagerter Kugelschalen

Grenzlasten unverschieblich gelenkig gelagerter Kugelschalen sind in Bild 6.9 angegeben. Im Gegensatz zu den auf die klassische Beullast bezogenen Grenzlasten 
nach DASt-Richtlinie 013 sind die bezogenen Grenzlasten gemäß Referenzwert der bezogenen Störenergie bei elastischem Materialverhalten von der Schlankheit abhängig. Die Plastifizierung des Schalenkontinuums bewirkt für mittelgroße bezogene Schlankheitsgrade $\bar{\lambda}_{s}$ eine signifikante Verringerung der Grenzlasten, wobei diese tendenziell gut mit denen nach den ECCS-Recommendations übereinstimmen. Somit ist dieses Regelwerk auch zur Beschreibung der Grenzlasten eingespannter und unverschieblich gelenkig gelagerter Kugelschalen unter Radialdruck geeignet.
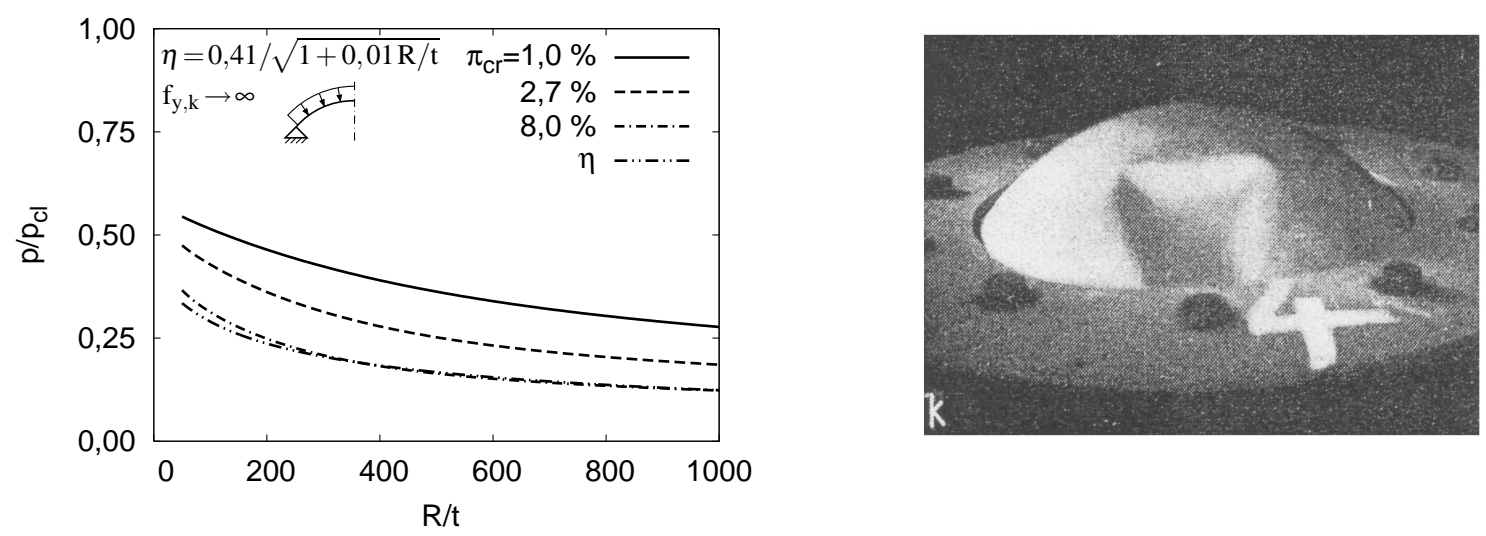

Bild 6.10: Experimentelle Erfahrungen vs. Störenergie-Konzept [122, 85]

Grenzlasten entsprechend der von Samuelson und Eggwertz [122] definierten $\eta$-Kurve sind in Bild 6.10 aufgeführt. Diese sind kleiner als die Grenzlasten gemäß Referenzwert der bezogenen Störenergie und zeigen in Abhängigkeit von der Schlankheit deutliche Unterschiede zu den Grenzlasten nach DASt-Richtlinie 013, vgl. Bild 6.9 links. Die Relevanz der lokalen Einzelbeule für die Stabilität von Kugelschalen unter Radialdruck bestätigen die Untersuchungen von Klöppel und Jungbluth [85].

\subsubsection{Radial verschieblich gelagerte Kugelschalen}

Die Ränder radial verschieblich gelagerter Kugelschalen können Verschiebungen in Richtung des Grundkreisradius oder des Kugelradius erfahren. Für den ersten Fall ist der Membranspannungszustand bei verschiedenen meridionalen Öffnungswinkeln bereits im Grundzustand signifikant gestört. Daher wird, wie Bild 6.11 für den Lastfall Radialdruck herausstellt, die Beullast mit abnehmendem Öffnungswinkel kleiner.

Die Steigung des Verlaufes der bezogenen Störenergie und die Grenzlast gemäß Referenzwert der bezogenen Störenergie verringern sich ebenfalls mit abnehmendem Öffnungswinkel. Für kleine Öffnungswinkel ist die bezogene Grenzlast nahezu linear von der Schlankheit abhängig. Für größere Öffnungswinkel ist diese Abhängigkeit deutlich nichtlinear. Die Verformungen infolge Störlast sind bei elastischem Materialverhalten für verschiedene Schlankheiten und Öffnungswinkel durch eine lokale Einzelbeule im Bereich des gelagerten Randes gekennzeichnet. Entsprechendes gilt für 

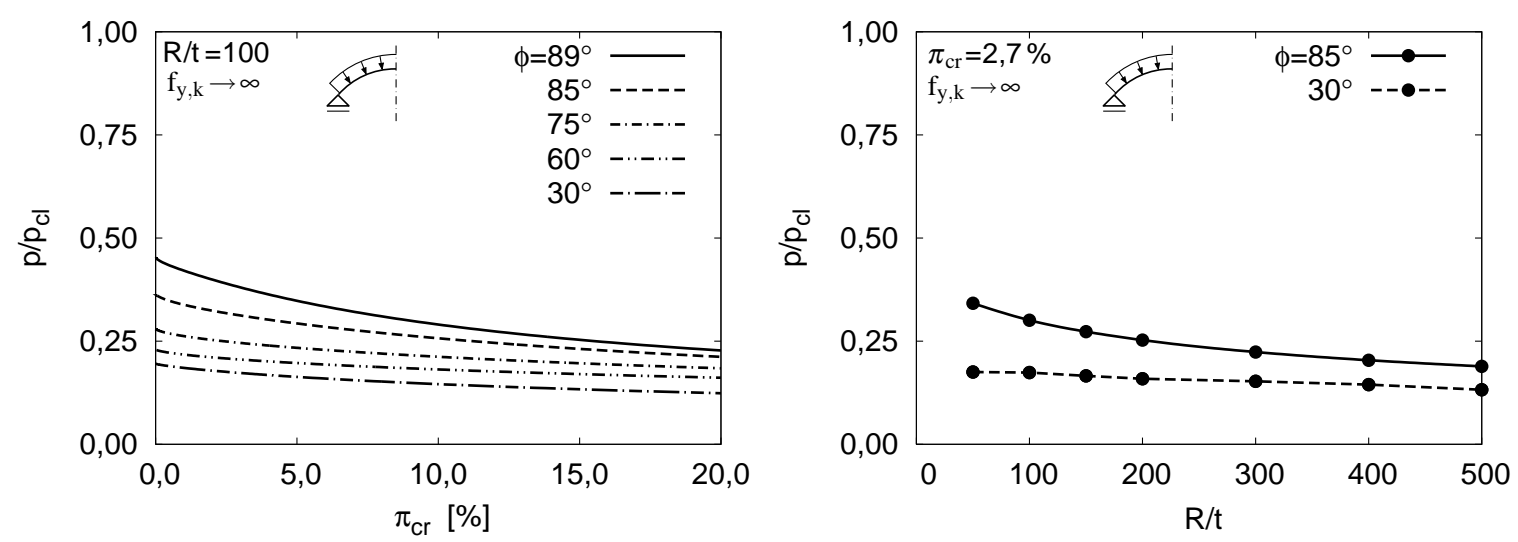

Bild 6.11: Zum Tragverhalten radial verschieblich gelagerter Kugelschalen

membrangerecht gelagerte Kugelschalen unter Radialdruck, gleichwohl diese nur bei kleinen Schlankheiten adäquat mit dem Störenergie-Konzept analysierbar sind.

\subsubsection{Kugelschalensegmente}

Kugelschalensegmente sind hinsichtlich der Ausdehnung in Meridian- und Breitenkreisrichtung sowie der Randbedingungen zu unterscheiden. Die Randbedingungen
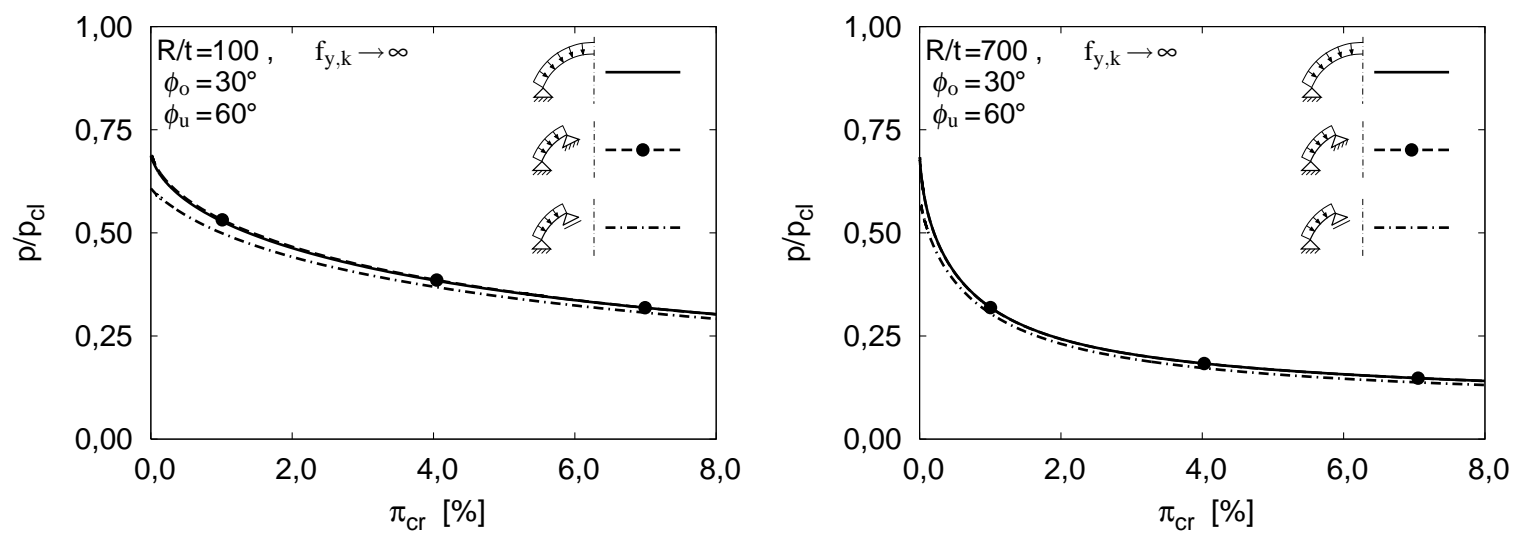

Bild 6.12: Imperfektionsempfindlichkeit ringförmiger Kugelschalensegmente

beeinflussen auch wesentlich den Membranspannungszustand der in Bild 6.12 dargestellten ringförmigen Kugelschalensegmente unter Radialdruck. Im Grundzustand ist für die am oberen Rand in Meridianrichtung verschieblich gelagerten Kugelschalensegmente die Membrandruckkraft in Breitenkreisrichtung betragsmäßig größer als in Meridianrichtung und größer als für die an beiden Rändern unverschieblich gelagerten Kugelschalensegmente. Der erste Beulfall weist die kleinere Beullast und die größere grundzustandspezifische Imperfektionsempfindlichkeit auf. Mit zunehmender Schlankheit werden die Unterschiede in der Imperfektionsempfindlichkeit geringer, die in den Verformungen infolge Störlast jedoch größer, vgl. Bild 6.13.

Für an beiden Rändern unverschieblich gelagerte Kugelschalensegmente sind dieVerformungen infolge Störlast unabhängig von der Schlankheit durch eine lokale Ein- 


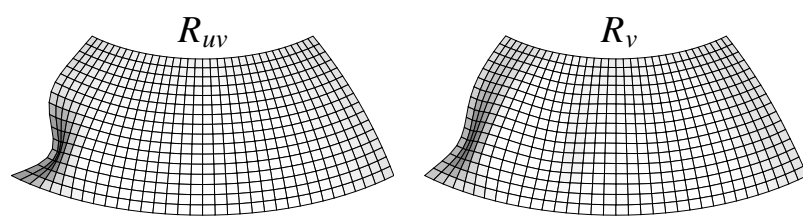

$R / t=100$
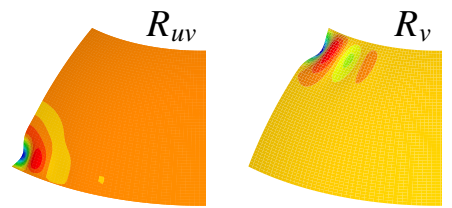

$R_{u v}$ 东

$R_{v} \gg$

Bild 6.13: Verformungen infolge Störlast vs. Randbedingungen und Schlankheit

zelbeule am unteren Rand charakterisiert. Für am oberen Rand in Meridianrichtung verschieblich gelagerte Kugelschalensegmente sind die Verformungen dagegen bei der Schlankheit $R / t=100$ am unteren Rand und bei $R / t=700$ am oberen Rand konzentriert. Dies ist mit der Verteilung der Membrandruckkräfte bzw. der Wölbfreiheit am oberen Rand zu begründen. Für beide Beulfälle sind die Grenzlasten gemäß Referenzwert der bezogenen Störenergie denen unverschieblich gelenkig gelagerter Kugelschalen mit unterschiedlichem Öffnungswinkel ähnlich, vgl. auch Bild 6.7.

Ist der obere Rand dagegen frei, sind bereits bei kleinen rotationssymmetrischen Öffnungen Beuleigenform und Verformungen infolge Störlast an diesem Rand erkennbar. Sowohl die Steigung des Verlaufes der bezogenen Störenergie als auch Beul- und Grenzlast nehmen entsprechend Bild 6.14 links mit größer werdender Öffnung ab.
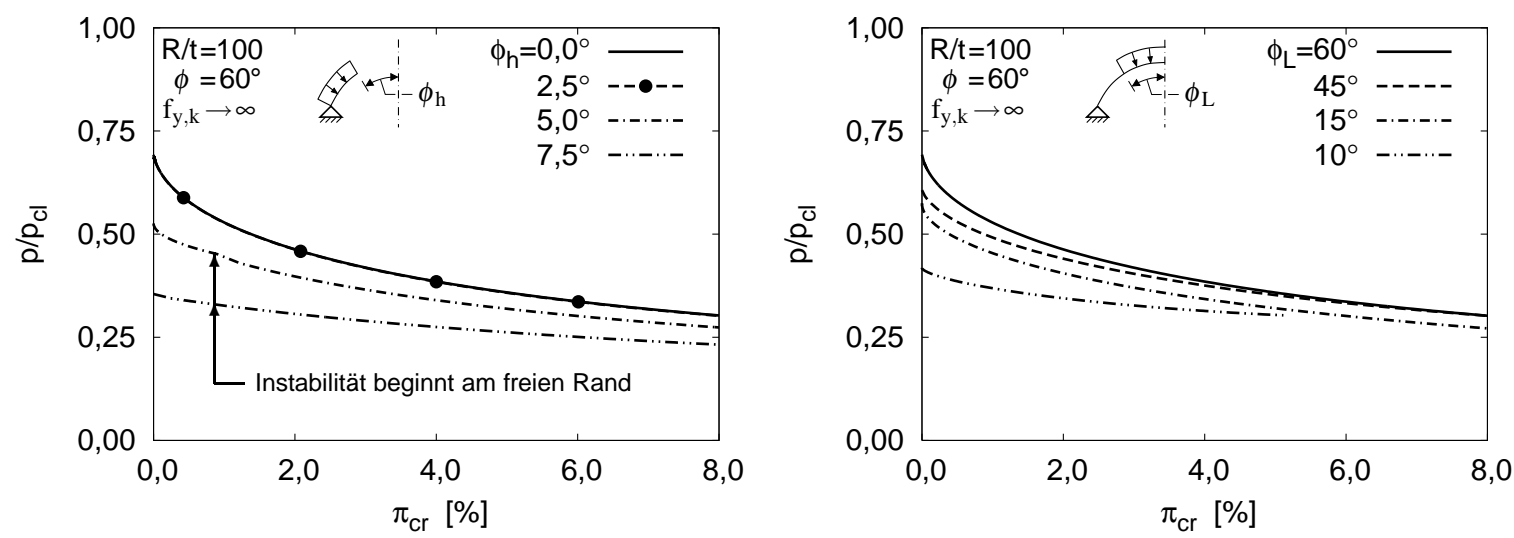

Bild 6.14: Imperfektionsempfindlichkeit verschiedener Beulfälle

Wirkt dagegen auf eine Kugelschale mit dem meridionalen Öffnungswinkel $\phi=60^{\circ}$ eine rotationssymmetrische Teilflächenbelastung ein, so verringern sich Beul- und Grenzlast mit abnehmender Belastungsfläche, vgl. Bild 6.14 rechts. Beuleigenform und Verformungen infolge Störlast sind für Öffnungswinkel $\phi_{\mathrm{L}}>10^{\circ}$ nicht rotationssymmetrisch und jeweils im Randbereich der Belastung konzentriert, da die dort für $\phi_{\mathrm{L}}<60^{\circ}$ auftretenden Störungen des Membranspannungszustandes gegenüber denen infolge der Randbedingungen überwiegen. Für den Öffnungswinkel $\phi_{L}=10^{\circ}$ sind beide Verformungsmoden rotationssymmetrisch und vornehmlich im Bereich des Scheitelpunktes der Kugelschale ausgeprägt, vgl. [48]. Bei kleineren Öffnungswinkeln sind kritische Zustände indes nur schwer zu identifizieren. 


\subsubsection{Vollkugelschalen}

Bei elastischem Materialverhalten sind die im Rahmen dieser Arbeit untersuchten Vollkugelschalen unter konstantem Radialdruck durch ein nahezu lineares Tragverhalten im Vorbeulbereich charakterisiert. In der Nähe der klassischen Beullast tritt eine Gruppierung singulärer Punkte auf. Eine in diesem Bereich ermittelte nicht rotationssymmetrische Beuleigenform ist in Bild 6.15 dargestellt.
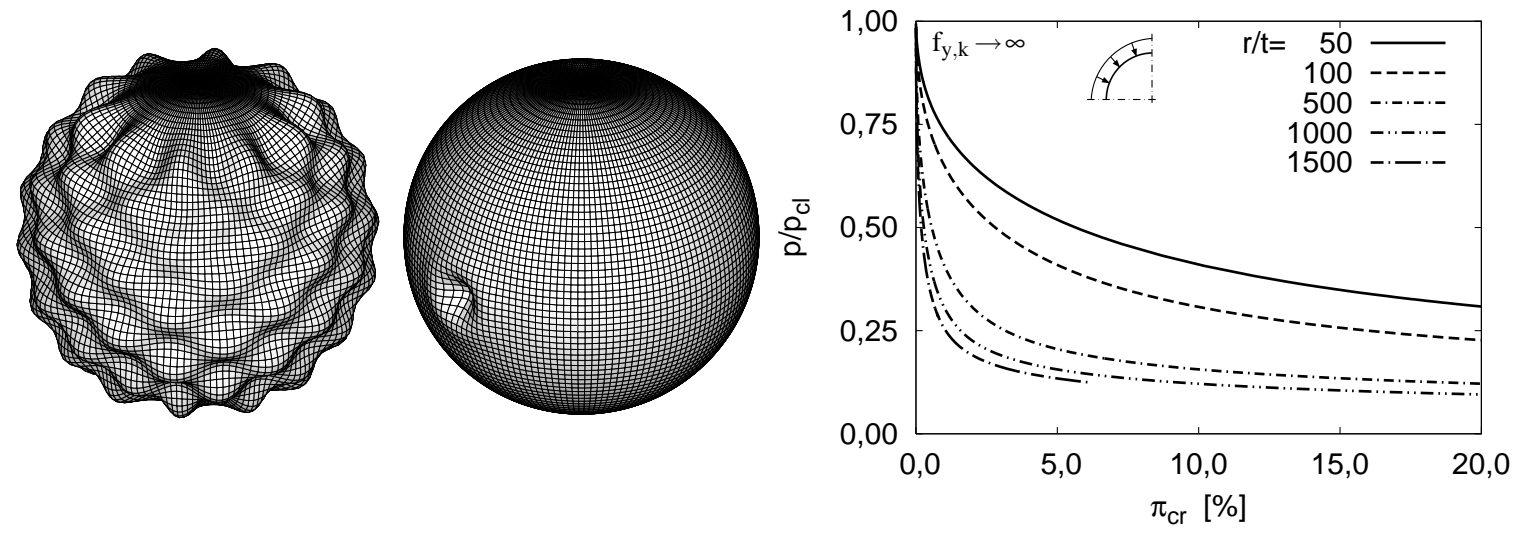

Bild 6.15: Beuleigenform und Verformungen infolge Störlast sowie bezogene Störenergie

Im Gegensatz zu unverschieblich gelagerten Kugelschalen erstreckt sich die Beuleigenform über nahezu die gesamte Fläche der Schale. Störlast und zugehörige Verformungen sind aber wiederum lokal konzentriert, wobei deren Position bei der numerischen Analyse durch die Symmetrierandbedingungen beeinflusst wird. Die Imperfektionsempfindlichkeit von Vollkugelschalen ist ebenfalls sehr groß und steigt für ein definiertes Lastniveau mit zunehmender Schlankheit an. Dabei nimmt die Differenz in der bezogenen Störenergie bei konstanter Änderung der Schlankheit ab.
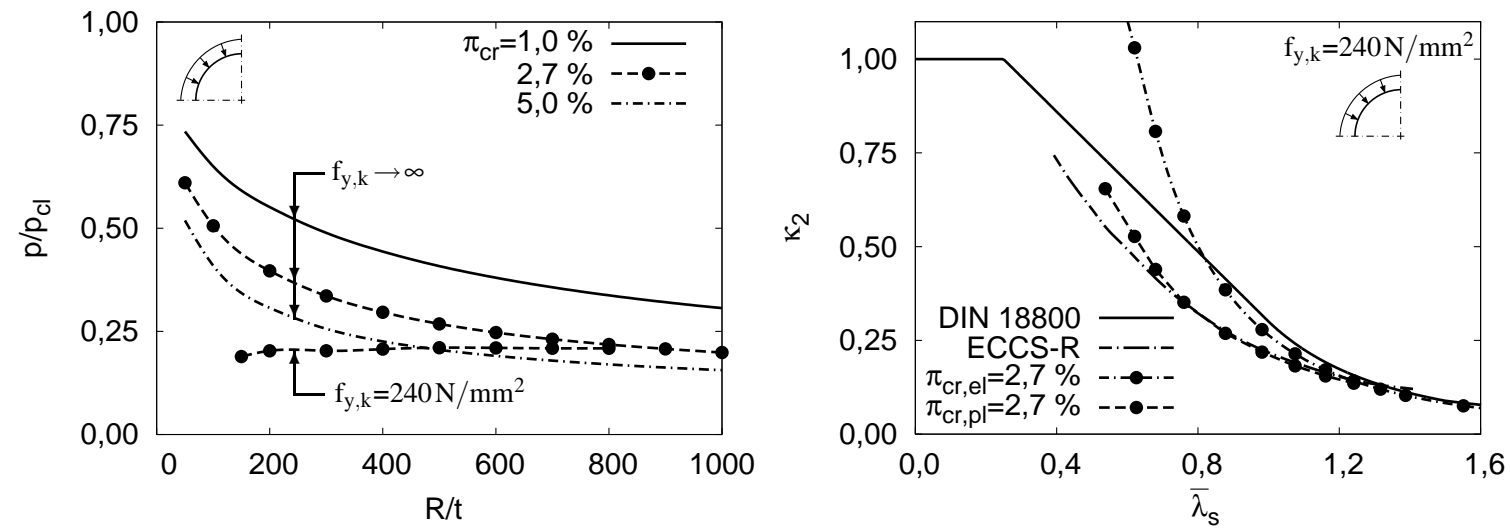

Bild 6.16: Grenzlasten von Vollkugelschalen unter Radialdruck

Während bei elastischem Materialverhalten die Schlankheit die bezogene Grenzlast signifikant beeinflusst, trifft dies entsprechend Bild 6.16 bei elastisch-plastischem Materialverhalten nicht zu. Für mittelgroße und große bezogene Schlankheitsgrade stimmen die Grenzlasten gemäß ECCS-Recommendations und Referenzwert der bezogenen Störenergie sehr gut überein, zeigen aber deutliche Unterschiede zu denen 
nach DIN 18800. Bemerkenswert ist die beim Vergleich der Bilder 6.16 und 6.9 nicht nur für elastisches Materialverhalten erkennbare Differenz zwischen den Grenzlasten nach DIN 18800 und denen gemäß Referenzwert der bezogenen Störenergie.

\subsection{Veränderliche Radialdruckbelastung}

Regelwerke konzentrieren sich auf wenige Beulfälle, z.B. auf Kugelschalen unter konstantem Radialdruck. Für Kugelschalen unter veränderlichem Radialdruck werden jedoch selten Hinweise gegeben. Das Störenergie-Konzept ermöglicht auch für derartige Beulfälle eine Bewertung der Imperfektionsempfindlichkeit und die Ermittlung von Grenzlasten mit einem energetisch äquivalenten Einfluss von Störungen.
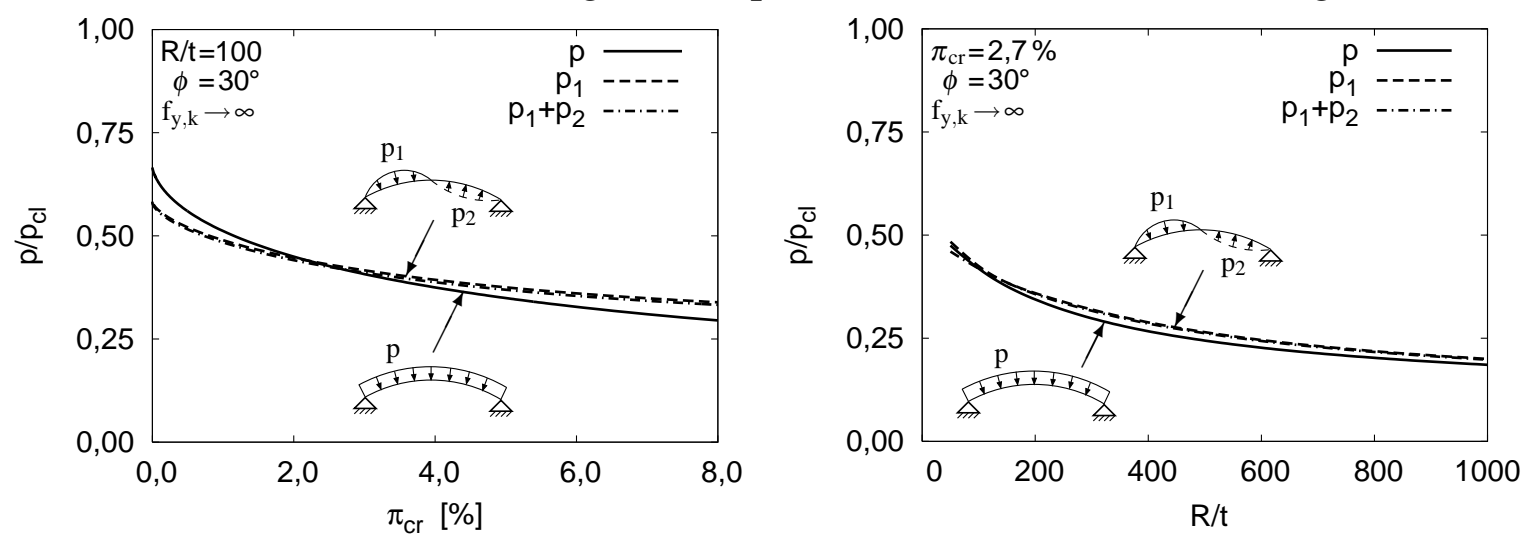

Bild 6.17: Imperfektionsempfindlichkeit und Grenzlasten vs. Grundbelastung

Nachfolgend werden unverschieblich gelenkig gelagerte Kugelschalen mit einem Öffnungswinkel $\phi=30^{\circ}$ untersucht, auf welche die Radialdrücke $p_{1}$ und $p_{2}$ einwirken, vgl. Bild 6.17. Beide Radialdrücke wirken halbseitig auf die Kugelschalen ein und sind in Breitenkreis- und Meridianrichtung mit einer Sinus-Halbwelle beschrieben. Zudem sind die Amplituden beider Radialdrücke betragsmäßig gleich und liegen der Darstellung der Ergebnisse zugrunde. Für die Kugelschalen unter veränderlichem Radialdruck sind Beullast und Steigung des Verlaufes der bezogenen Störenergie kleiner als für Kugelschalen unter konstantem Radialdruck. Das Verhältnis der bezogenen Störenergien ist indes vom Lastniveau abhängig. Der für die nicht rotationssymmetrischen Belastungen geringe Unterschied in Beullast und Imperfektionsempfindlichkeit zeigt, dass Radialdruck $p_{2}$ kaum destabilisierend wirkt. Die Grenzlast ist nur für sehr gedrungene Kugelschalen unter Radialdruck $p_{1}+p_{2}$ geringer als bei konstantem Radialdruck. Verformungen im kritischen Zustand $M$ sind in Bild 6.18 zehnfach überhöht dargestellt. Die Verformungen sind nicht rotationssymmetrisch und mit zunehmender Unsymmetrie der Belastung stärker ausgeprägt.

Im Gegensatz zu Kugelschalen unter konstantem Radialdruck sind bei veränderlichem Radialdruck die Imperfektionsempfindlichkeit und die Grenzlasten zumindest 


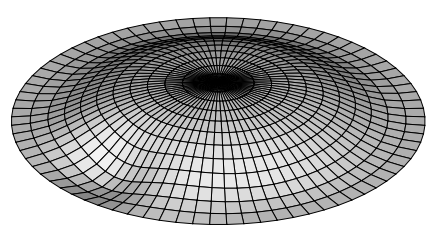

$p$

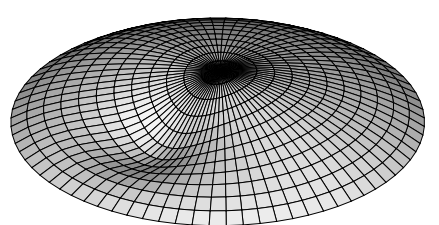

$p_{1}$

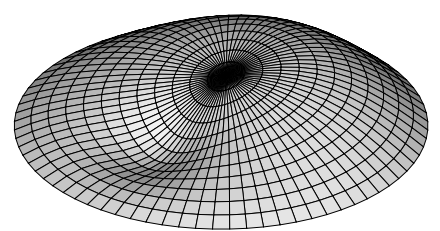

$p_{1}+p_{2}$

Bild 6.18: Verformungen im kritischen Zustand vs. Grundbelastung $\left(p / p_{c l}=0,56\right)$

für kleine Schlankheiten merklich vom Öffnungswinkel abhängig, vgl. Bild 6.19. Dabei sind mit zunehmendem Öffnungswinkel das Maximum der Membrandruckkräfte und die Verformungen infolge Störlast weiter vom gelagerten Rand entfernt. Für die Schlankheit $R / t=400$ ist der Einfluss des Öffnungswinkels auf die Beullast und den Verlauf der bezogenen Störenergie indes bereits sehr gering.
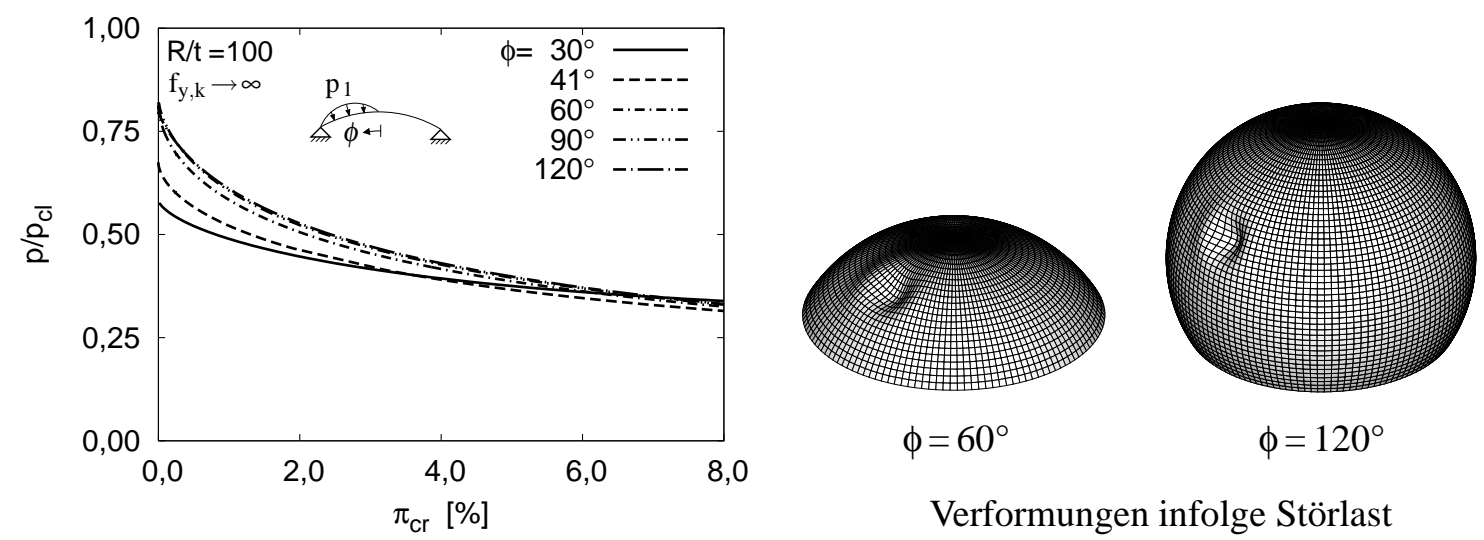

Verformungen infolge Störlast

Bild 6.19: Tragverhalten vs. meridionaler Öffnungswinkel

Einen weiteren interessanten Beulfall repräsentieren Kugelschalen unter eigengewichtsaffiner Belastung. Bei diesem Beulfall nimmt die in Meridianrichtung wirkende Membrandruckkraft mit ansteigendem Öffnungswinkel betragsmäßig zu und ist die in Breitenkreisrichtung wirkende Membrankraft für Öffnungswinkel $\phi>51,8^{\circ}$ bereichsweise positiv [60]. Beullast und bezogene Störenergie sind ebenfalls signifikant vom Öffnungswinkel abhängig. Für unverschieblich gelenkig gelagerte Kugelschalen mit elastischem Materialverhalten und dem Öffnungswinkel $\phi=30^{\circ}$ sind die Grenzlasten gemäß Referenzwert der bezogenen Störenergie bei eigengewichtsaffiner Belastung um etwa $10 \%$ größer als bei konstantem Radialdruck, vgl. Bild 6.9.

\subsection{Bewertung des Tragverhaltens von Kugel- und Kreiszylinderschalen}

Kugel- und Kreiszylinderschalen weisen für ausgewählte Belastungen und Randbedingungen ähnliches Tragverhalten auf. Dies verdeutlichen nicht nur die ggf. vorhandene Gleichheit der idealen Beulspannungen und Ähnlichkeit des Verzweigungsverhaltens [103], sondern hinsichtlich der Imperfektionsempfindlichkeit auch die in Bild 6.20 angegebenen Verläufe der bezogenen Störenergie. 

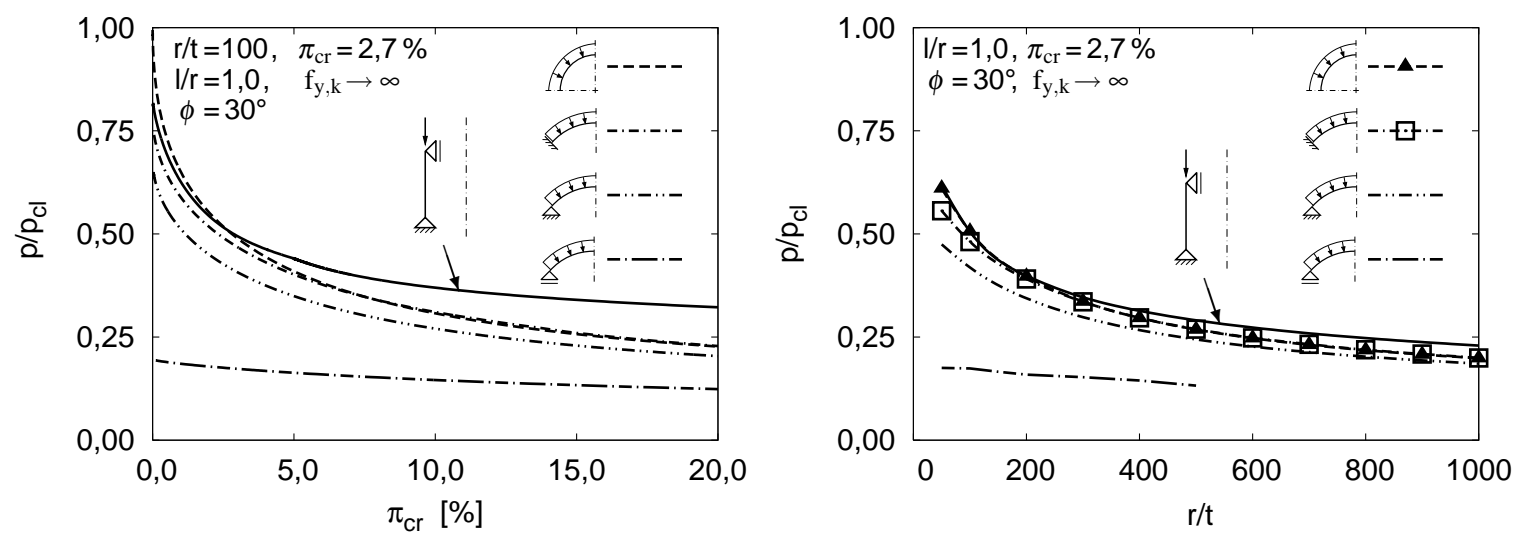

Bild 6.20: Imperfektionsempfindlichkeit und Grenzlasten verschiedener Beulfälle

Für höhere Lastniveaus besitzen Vollkugel- und unverschieblich gelagerte Kugelschalen unter Radialdruck eine annähernd gleiche Steigung des Verlaufes der bezogenen Störenergie wie axialgedrückte Kreiszylinderschalen. Für ein definiertes Lastniveau sind die Kugelschalen aber oftmals imperfektionsempfindlicher als die Kreiszylinderschalen. Die Unterschiede in der Imperfektionsempfindlichkeit verringern sich mit zunehmender Schlankheit nur geringfügig. Die auf die jeweilige klassische Beullast bezogene Beullast ist für die Vollkugelschalen, das bezogene Nachbeulminimum hingegen für die Kreiszylinderschalen maximal. Ein Vergleich mit Bild 5.1 zeigt die im Gegensatz zu am belasteten Rand freien Kreiszylinderschalen geringe Imperfektionsempfindlichkeit verschieblich gelagerter flacher Kugelschalen auf.

Bei elastischem Materialverhalten stimmen Bild 6.20 zufolge die mit dem Referenzwert der bezogenen Störenergie definierten Grenzlasten von Vollkugel- und eingespannten Kugelschalen gut überein. Mit zunehmender Schlankheit verringern sich die Unterschiede zwischen diesen Grenzlasten und denen unverschieblich gelenkig gelagerter Kugelschalen. Die größte bezogene Grenzlast besitzen nahezu unabhängig von der Schlankheit die Kreiszylinderschalen. Die Grenzlasten radial verschieblich gelagerter Kugelschalen sind deutlich geringer als die der oben diskutierten Beulfälle und darüber hinaus signifikant vom meridionalen Öffnungswinkel abhängig. Dies wird in den Regelwerken oft nicht hinreichend berücksichtigt. Entsprechendes gilt für die Regelungen der DASt-Richtlinie 013 zum Einfluss der Schlankheit auf die Grenzlasten von Kugelschalen mit elastischem Materialverhalten.

Für elastisch-plastisches Materialverhalten sind Grenzlasten eingespannter Kugelschalen unter Radialdruck und axialgedrückter Kreiszylinderschalen in Bild 6.21 links gegenübergestellt. Die Grenzlasten gemäß Referenzwert der bezogenen Störenergie liegen bei mittelgroßen bezogenen Schlankheitsgraden für Kugelschalen teilweise deutlich unterhalb und für Kreiszylinderschalen oberhalb der Grenzlasten nach DIN 18800 [87]. Die Unterschiede resultieren aus dem in DIN 18800 gewählten Ansatz, die Grenzlasten beider Beulfälle mit einer Funktion zu approximieren, verdeut- 
lichen aber die Schwierigkeiten bei der Identifikation der tatsächlichen Grenzlast eines Schalentragwerkes. Bessere Übereinstimmung zeigen die Grenzlasten gemäß Referenzwert der bezogenen Störenergie mit denen nach den ECCS-Recommendations.
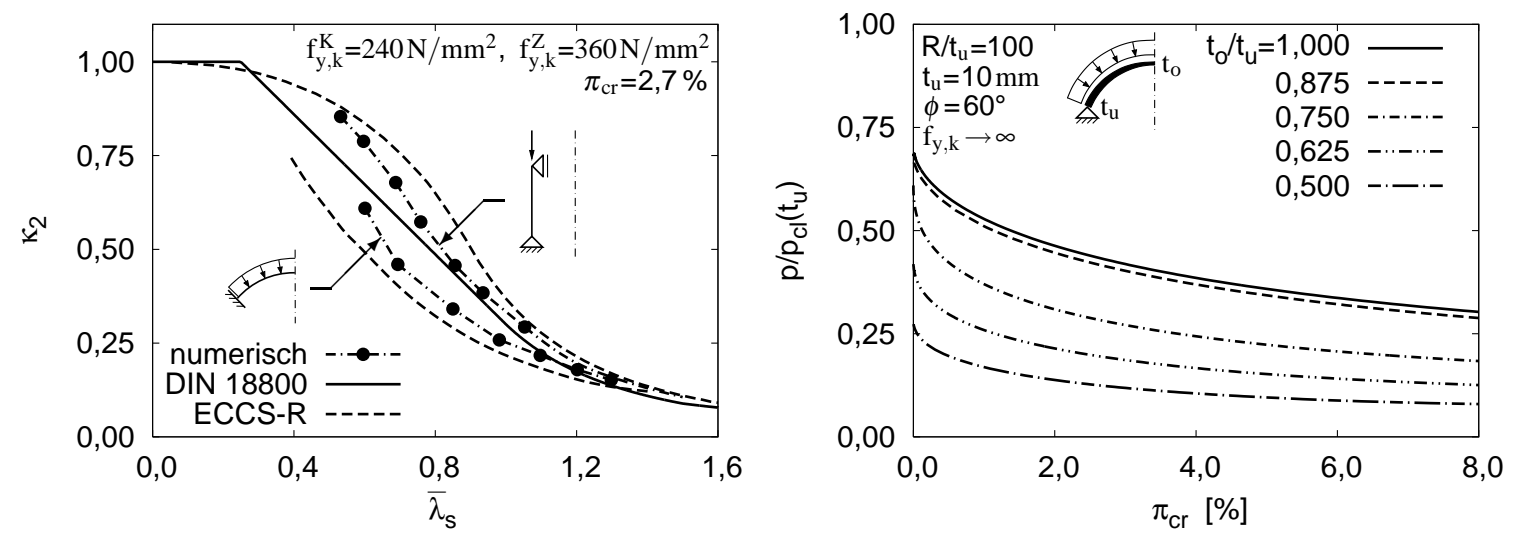

Bild 6.21: Grenzlasten und Imperfektionsempfindlichkeit vs. Beulfall

Die mittels des Referenzwertes der bezogenen Störenergie angegebenen realistischen Grenzlasten von Kugel- und Kreiszylinderschalen basieren auf der Ähnlichkeit der Störlasten und der praktischen Relevanz der lokalen Einzelbeule. Daher wäre in Anlehnung an Kegelschalen unter Meridianlast die Definition einer Ersatz-Kreiszylinderschale für Kugelschalen unter Radialdruck denkbar [87].

Abstufungen der Wanddicke können das Tragverhalten von Schalentragwerken signifikant beeinflussen. Dieser Aspekt wird für zweischüssige Kreiszylinderschalen unter Axiallast in [144] erörtert. Für Kugelschalen unter Radialdruck wird nachfolgend exemplarisch der Grenzfall einer in Meridianrichtung stetig veränderlichen Wanddicke betrachtet. Bild 6.21 rechts zeigt, dass für verschiedene Wanddickenverhältnisse $t_{o} / t_{u}$ der Verlauf der bezogenen Störenergie eine ähnlich große Steigung aufweist. Die Verformungen infolge Störlast sind im Gegensatz zu denen axialgedrückter Kreiszylinderschalen wesentlich von der Änderung der Wanddicke abhängig. Für $t_{o} / t_{u} \geq$ 0,875 sind die Verformungen nicht rotationssymmetrisch und am gelagerten Rand, andernfalls rotationssymmetrisch und im Bereich des Scheitelpunktes konzentriert. Die bei einer stetig veränderlichen Wanddicke erforderliche lokale Normierung der Störenergie verdeutlicht, dass globale Faktoren nur bedingt zur Bewertung des Einflusses ausgewählter Parameter auf die Imperfektionsempfindlichkeit geeignet sind. 


\section{Statische Grenzlasten ausgewählter Schalenbeulfälle}

Die Imperfektionsempfindlichkeit und die Grenzlasten von Schalentragwerken sind auch von der Gauß'schen Krümmung abhängig. Die Krümmung ist bei Einzelschalen konstant oder stetig veränderlich, kann aber bei zusammengesetzten Schalen diskontinuierliche Änderungen erfahren und das Tragverhalten entscheidend beeinflussen.

\subsection{Kegelschalen unter Meridianlast}

Für in Meridianrichtung druckbeanspruchte Kegelschalen mit konstanter Meridianlänge $l$ und konstantem Radius $r_{o}$ am oberen Rand verdeutlicht Bild 7.1 den Einfluss des Öffnungswinkels $\rho$ auf die Beullast.
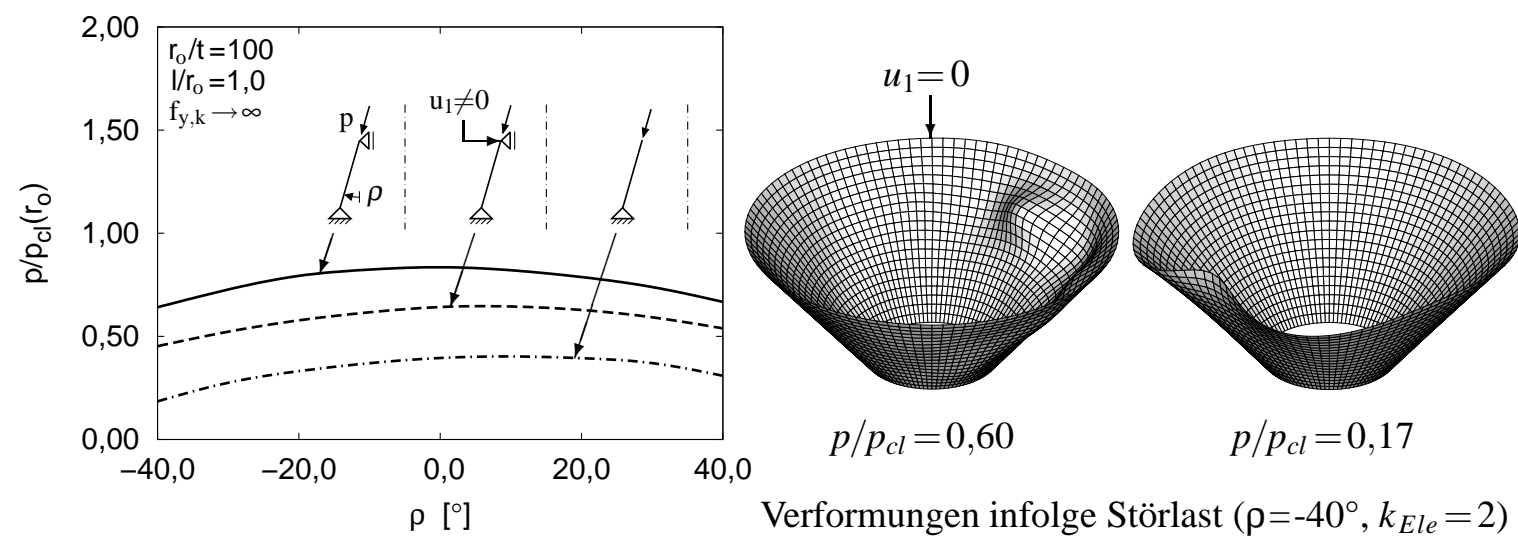

Bild 7.1: Tragverhalten vs. Randbedingungen ( $p_{c l}$ nach Gl. (5.1))

Sind beide Ränder in Radial- und Umfangsrichtung unverschieblich gelagert, so ist die Beullast mit dem Kosinus des Öffnungswinkels zu beschreiben. Werden am belasteten Rand Verschiebungen $u_{1}$ in Umfangsrichtung zugelassen, verringert sich die Beullast merklich. Gleichzeitig stellt sich die maximale Beullast wie bei am belasteten Rand freien Kegelschalen für einen kleinen positiven Öffnungswinkel ein. Beuleigenform und die vornehmlich nach innen gerichteten Verformungen infolge Störlast sind für die ersten beiden Beulfälle im Bereich des wölbfreien Randes und für den dritten Beulfall unmittelbar am freien Rand konzentriert.

Für den ersten und den dritten Beulfall sind in Bild 7.2 Verläufe der bezogenen Störenergie angegeben. Es ist ersichtlich, dass die Steigung der Verläufe für beide Beul- 

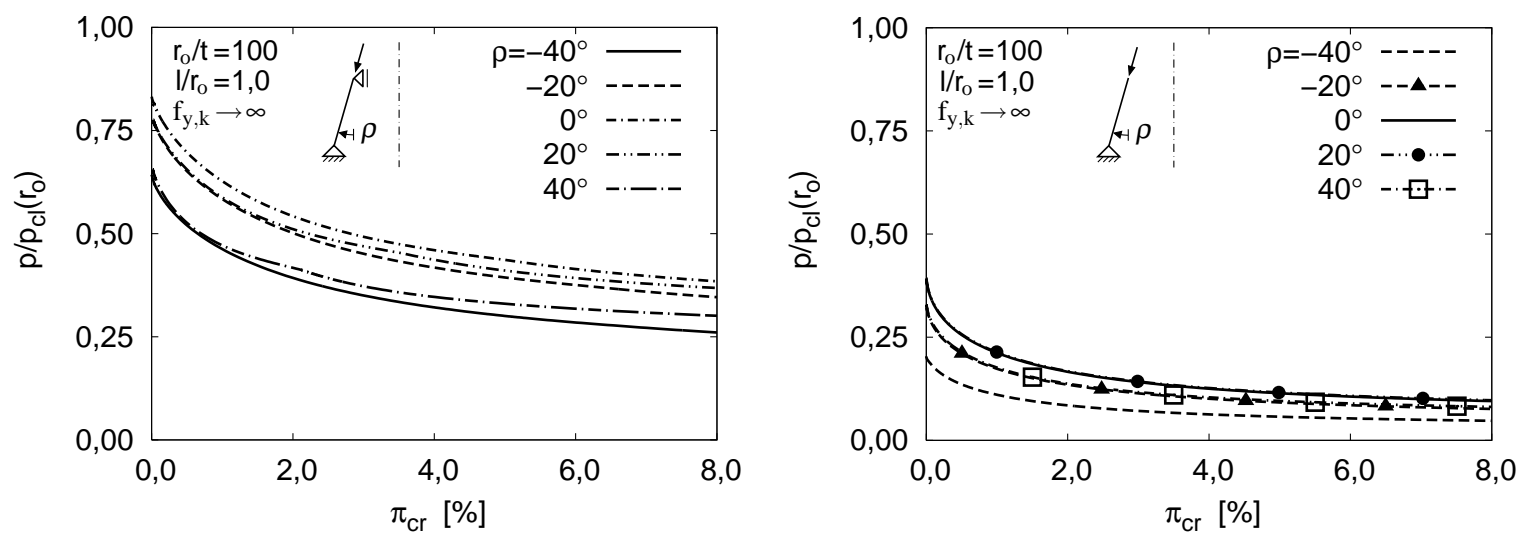

Bild 7.2: Imperfektionsempfindlichkeit vs. Öffnungswinkel

fälle nur unwesentlich vom Öffnungswinkel abhängig ist. Dennoch sind die jeweiligen Verläufe nicht mit dem Kosinus des Öffnungswinkels ineinander überführbar. Diskontinuitäten in der Steigung der Verläufe repräsentieren kleine Änderungen in Form und Position der Verformungen infolge Störlast. Für die am belasteten Rand in Radial- und Umfangsrichtung unverschieblich gelagerten Kegelschalen sind Unterschiede im Verlauf der bezogenen Störenergie für betragsmäßig gleiche, aber im Vorzeichen differierende Öffnungswinkel auch mit zunehmender Schlankheit noch erkennbar. Dabei liegen im Bereich des Referenzwertes der bezogenen Störenergie die Verläufe für positive Öffnungswinkel über denen für betragsmäßig gleiche, aber negative Öffnungswinkel. Ferner nimmt für ein definiertes Lastniveau die bezogene Störenergie mit ansteigendem Betrag des Öffnungswinkels oftmals ab.

Grenzlasten von Kegelschalen unter konstanter Meridianlast sind in Bild 7.3 aufgezeigt, wobei den Darstellungen der Radius $r_{o} / \cos \rho$ der Ersatz-Kreiszylinderschale zugrunde liegt. Dieser Radius beschreibt die Position der Verformungen infolge Störlast für verschiedene Randbedingungen zwar nicht exakt, erlaubt es aber, das Stabilitätsverhalten der untersuchten Kegelschalen hinreichend genau zu erfassen.
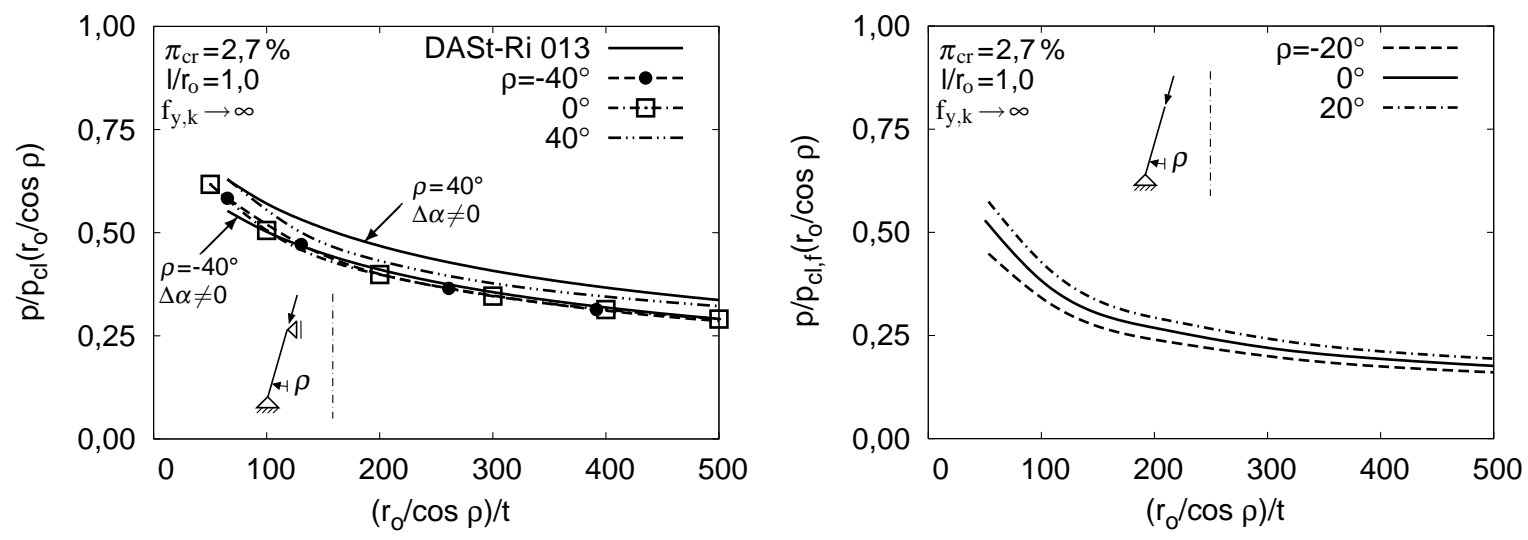

Bild 7.3: Grenzlasten vs. Öffnungswinkel und Schlankheit

Für am belasteten Rand in Radial- und Umfangsrichtung unverschieblich gelagerte Kegelschalen stimmen bei dem Öffnungswinkel $\rho=-40^{\circ}$ die Grenzlasten gemäß Re- 
ferenzwert der bezogenen Störenergie mit denen nach DASt-Richtlinie 013 gut überein [87]. Die angegebenen Grenzlasten des Regelwerkes berücksichtigen die zulässige Erhöhung des Abminderungsfaktors $\alpha$ um den Wert $\Delta \alpha$, aber nicht den zusätzlichen Abminderungsfaktor 0,75. Bei dem Öffnungswinkel $\rho=40^{\circ}$ sind die Grenzlasten gemäß Referenzwert der bezogenen Störenergie merklich kleiner als die nach DASt-Richtlinie 013. Für am belasteten Rand freie Kegelschalen sind die angegebenen und auf die klassische Beullast $p_{c l, f}$ gemäß Abschnitt 5.1 bezogenen Grenzlasten auch von dem Öffnungswinkel und der Schlankheit abhängig. Ein Vergleich dieser Grenzlasten mit denen nach einem Regelwerk ist nicht möglich, da sich die einschlägigen Regelwerke nur auf radial unverschieblich gelagerte Kegelschalen beziehen.

Bei in Umfangsrichtung veränderlicher Meridianlast $p(\varphi)=\widehat{p} \cos \varphi$ ist die Beullast von am belasteten Rand in Radial- und Umfangsrichtung unverschieblich gelagerten Kegelschalen wiederum mit dem Kosinus des Öffnungswinkels beschreibbar. Für die Schlankheit $r / t=100$ ist die Beullast um ca. $8 \%$ größer als bei gleichartigen Kegelschalen unter konstanter Meridianlast. Der Unterschied verringert sich jedoch mit zunehmender Schlankheit. Beuleigenform und Verformungen infolge Störlast sind im Bereich des gestauchten belasteten Randes konzentriert. Beide Aspekte folgen aus der raschen Änderung der Zustandsgrößen in Umfangsrichtung [122].
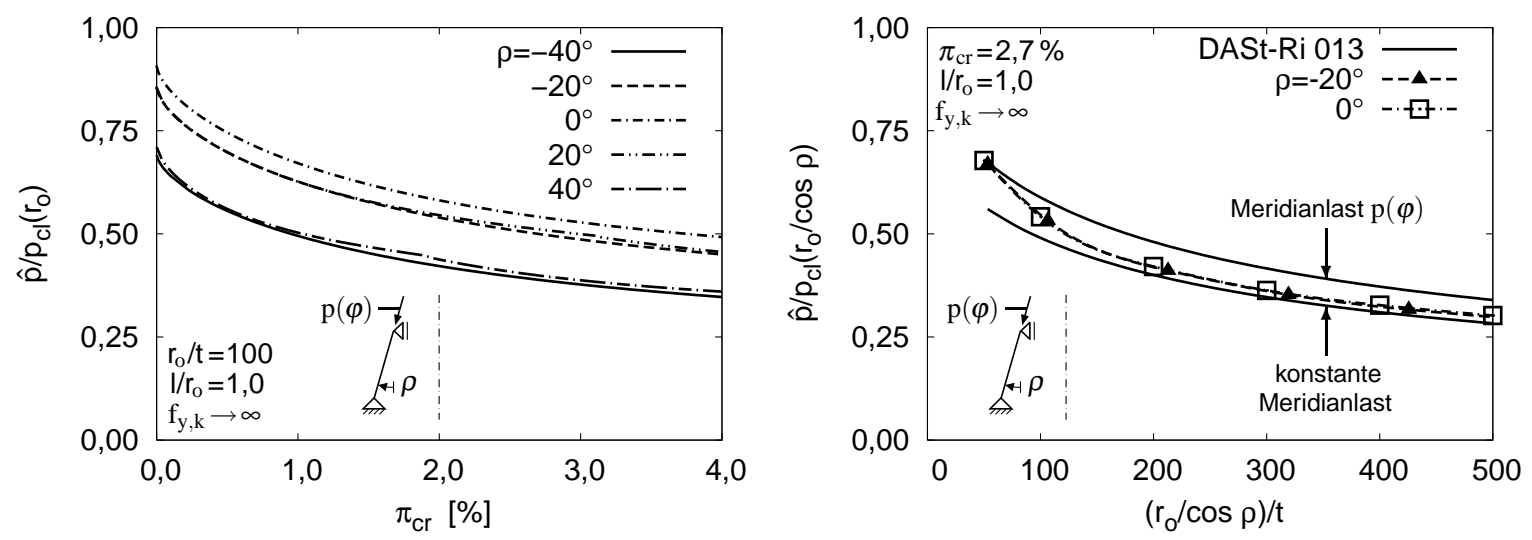

Bild 7.4: Tragverhalten vs. Öffnungswinkel und Schlankheit

Die Steigung des Verlaufes der bezogenen Störenergie ist, wie ein Vergleich der Bilder 7.2 und 7.4 zeigt, nur geringfügig von der Verteilung der Belastung in Umfangsrichtung abhängig. Die Grenzlasten gemäß Referenzwert der bezogenen Störenergie sind für die angegebenen Schlankheiten und Öffnungswinkel den Grenzlasten nach DASt-Richtlinie 013 für konstante Meridianlast ähnlich. Insofern ist die in diesem Regelwerk bei reiner Biegebeanspruchung von Kreiszylinder- und Kegelschalen zulässige Erhöhung der Grenzlasten um 20\% zumindest für schlanke Schalentragwerke mit elastischem Materialverhalten nicht zu rechtfertigen [103]. Kegelschalen unter Außendruck weisen eine geringe Imperfektionsempfindlichkeit auf und sind daher nur bedingt mit dem Störenergie-Konzept analysierbar. 


\subsection{Rotationsparaboloide unter verschiedenen Belastungen}

An Rotationsschalen, deren Erzeugende mit einer ganzrationalen Funktion zweiten Grades spezifiziert ist, wird der Einfluss der Gauß'schen Krümmung auf das Tragverhalten verdeutlicht. Bei in Radial- und Umfangsrichtung unverschieblich gelagerten Rändern und Axiallast bewirkt Bild 7.5 zufolge ein ansteigendes Radienverhältnis $r_{m} / r_{o}$ eine Zunahme der Beul- und der Grenzlast. Gleichzeitig verringern sich für höhere Lastniveaus die Imperfektionsempfindlichkeit und die Steigung des Verlaufes der bezogenen Störenergie merklich. Dies folgt aus den stabilisierend wirkenden Umfangsmembrankräften und den Randstörungen am belasteten Rand.
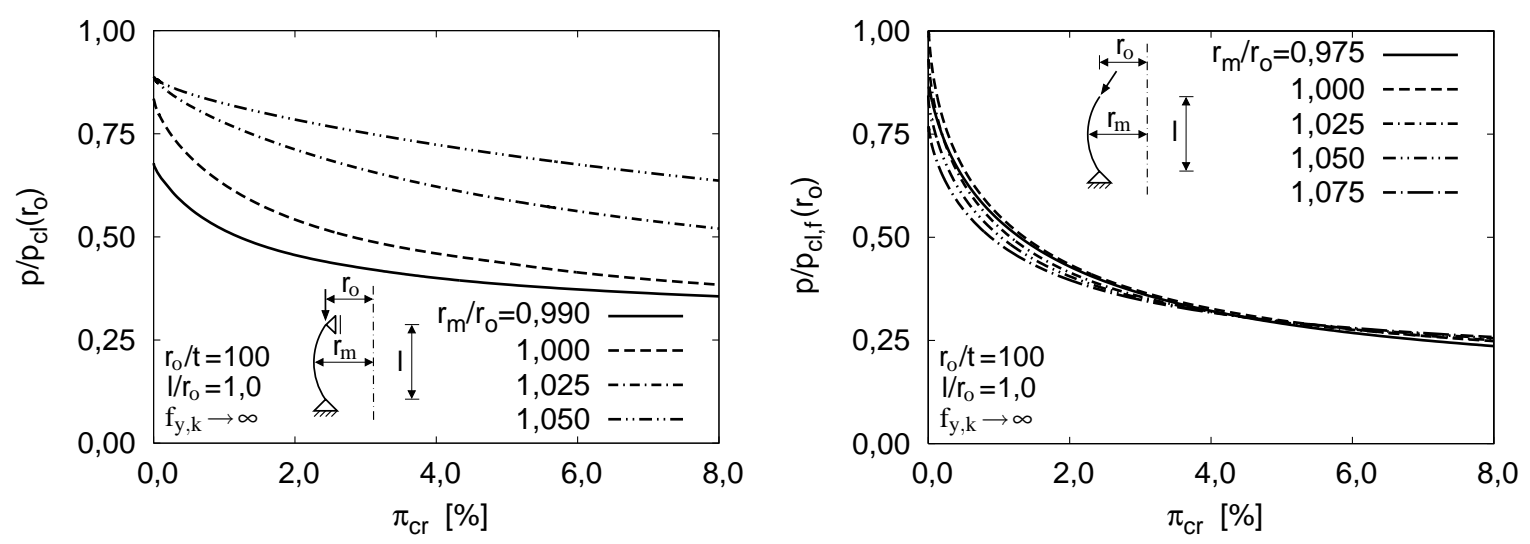

Bild 7.5: Imperfektionsempfindlichkeit vs. Gauß'sche Krümmung

Ist der belastete Rand frei und wirkt auf den Rotationsparaboloiden eine Meridianlast ein, so wird die Steigung des Verlaufes der bezogenen Störenergie nur geringfügig von den angegebenen Radienverhältnissen beeinflusst. Für das Lastniveau $p / p_{c l, f}=$ 0,31 , wobei $p_{c l, f}$ wiederum die klassische Beullast gemäß Abschnitt 5.1 repräsentiert, ist die Imperfektionsempfindlichkeit nahezu unabhängig vom Radienverhältnis.

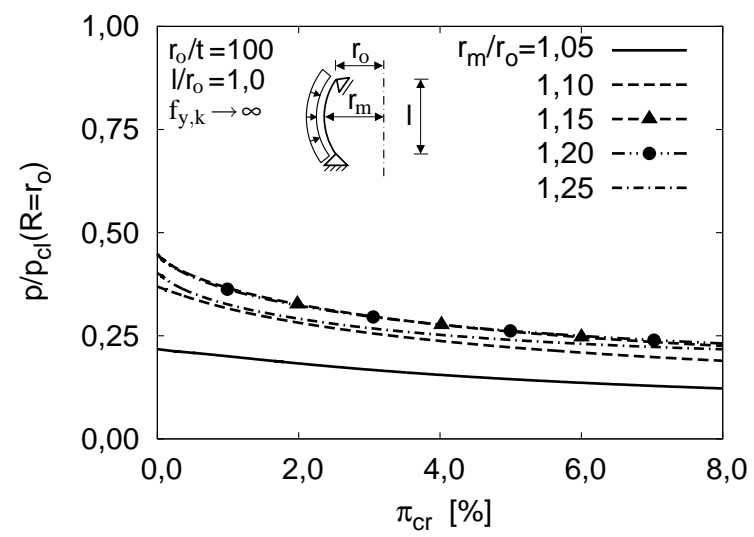

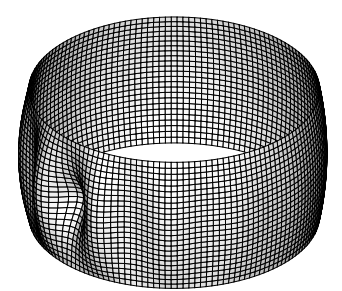

$r_{m} / r_{o}=1,05$

Verformungen infolge Störlast

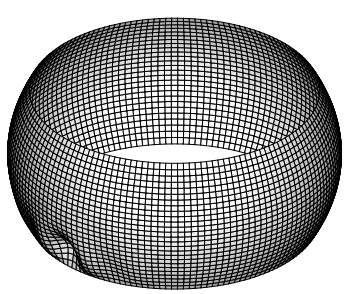

$r_{m} / r_{o}=1,15$

Bild 7.6: Tragverhalten vs. Gauß'sche Krümmung ( $p_{c l}$ nach Gl. (6.1))

Für den in Bild 7.6 dargestellten Beulfall erhöhen sich bei ansteigendem Radienverhältnis zunächst die Beullast und für ein definiertes Lastniveau die bezogene Störenergie. Ursache hierfür ist der zunehmend zweidimensionale Membranspannungszu- 
stand. Überschreitet das Radienverhältnis einen bestimmten Wert, erfolgt der Lastabtrag zunehmend über Biegung und die Grenzlast wird kleiner. Die Verformungen infolge Störlast sind je nach Radienverhältnis über die gesamte Meridianlänge ausgedehnt oder am unteren Rand konzentriert. In Umfangsrichtung klingen die Verformungen unabhängig vom Radienverhältnis rasch ab. Zusammenfassend wird der beulfallspezifische Einfluss der Gauß'schen Krümmung auf das Tragverhalten deutlich.

\subsection{Zusammengesetzte Schalen unter Radialdruck}

Die Imperfektionsempfindlichkeit und die Grenzlasten zusammengesetzter Schalen sind von der Geometrie und der Belastung der Teilschalen, aber auch von der konstruktiven Durchbildung des Überganges zwischen den Teilschalen abhängig. Für nicht ausgesteifte Zylinder-Kugelschalen-Konfigurationen mit einem meridionalen Öffnungswinkel von $\phi=45^{\circ}$ bzw. $90^{\circ}$ und einer Radialdruckbelastung, die nur auf die Kugelschale einwirkt, sind in Bild 7.7 links die Verformungen infolge Störlast dargestellt. Die Verformungen sind für beide Konfigurationen durch eine lokale Einzelbeule charakterisiert. Für den kleineren Öffnungswinkel ist die Einzelbeule im Bereich der Kugelschale konzentriert, wohingegen bei dem größeren Öffnungswinkel die Verformungen beide Teilschalen erfassen.

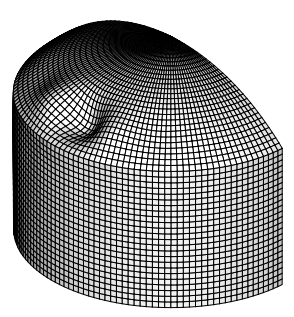

$p / p_{c l}=0,40$

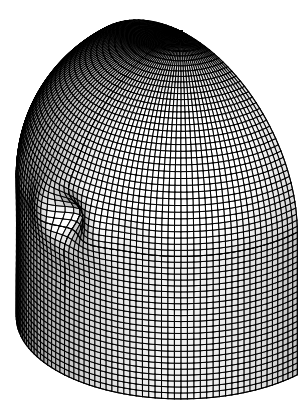

$p / p_{c l}=0,59$

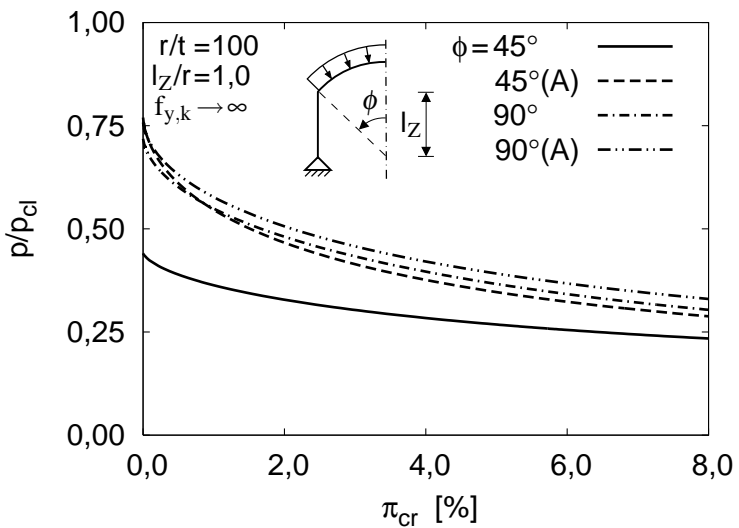

Bild 7.7: Verformungen infolge Störlast und Imperfektionsempfindlichkeit

Die Steigung des Verlaufes der bezogenen Störenergie und die Grenzlast sind, wie Bild 7.7 rechts bei Normierung der Belastung auf die klassische Beullast der jeweiligen Kugelschale aufzeigt, für die Konfiguration mit dem kleineren Öffnungswinkel deutlich kleiner als für die mit dem größeren Öffnungswinkel. Dies ist mit den unterschiedlichen Störungen des Membranspannungszustandes am Übergang zwischen den Teilschalen zu begründen. Von Interesse ist das Wissen um die stabilisierende Wirkung von Aussteifungen, die am Übergang zwischen den Teilschalen angeordnet sind. Der Grenzfall ringförmiger Aussteifungen (A) unendlich großer Steifigkeit, die mit Verschiebungsfesthaltungen in Radialrichtung modelliert sind, verdeutlicht, in- 
wiefern mit der Anordnung von Aussteifungen für beide Konfigurationen die Beulund Grenzlast sowie die Imperfektionsempfindlichkeit beeinflusst werden können.
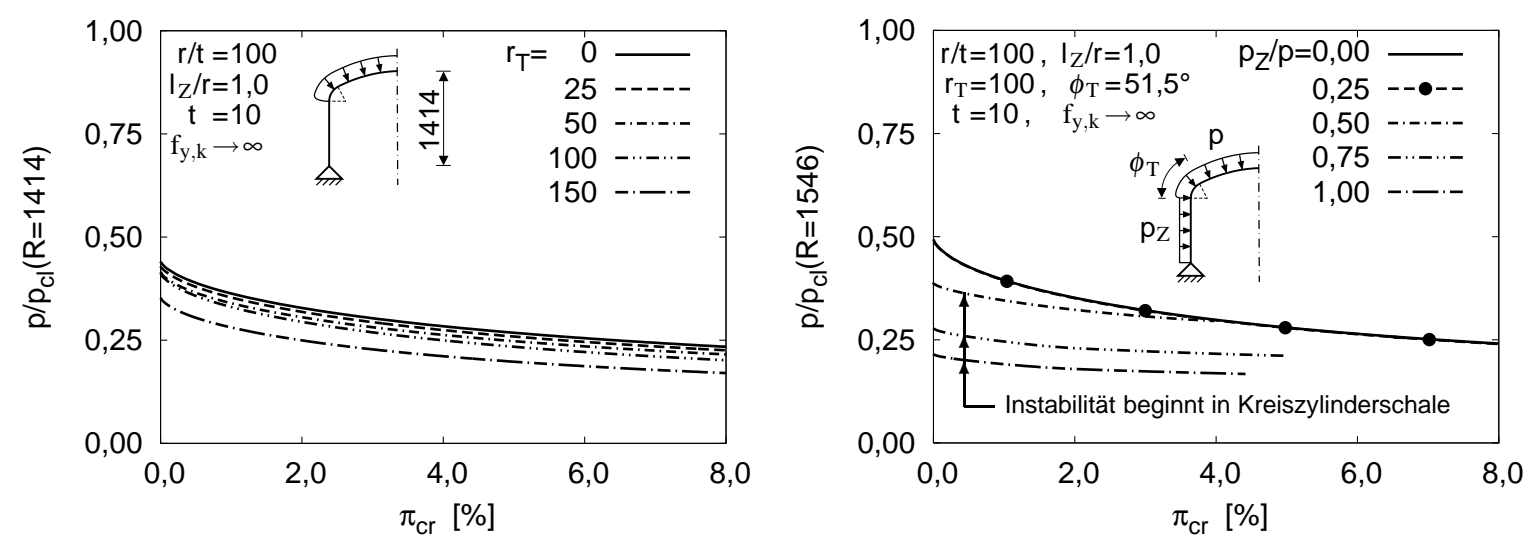

Bild 7.8: Imperfektionsempfindlichkeit vs. Beulfall

Wird dagegen der Übergang zwischen den Teilschalen torusförmig ausgerundet, so nimmt bei konstanter Höhe des Schalentragwerkes und ansteigendem Ausrundungsradius $r_{T}$ die Schlankheit der Kugelschale zu, vgl. Bild 7.8 links. Demzufolge verringern sich Beul- und Grenzlast. Die Steigung des Verlaufes der bezogenen Störenergie bleibt jedoch nahezu unverändert. Die Länge $l_{Z}$ der Kreiszylinderschale beeinflusst das Tragverhalten nur unwesentlich. Ist zusätzlich zur Kugelschale auch die Kreiszylinderschale durch Radialdruck belastet und somit deren Membranspannungszustand zweidimensional, kann, wie in Bild 7.8 rechts dargestellt, Instabilität in Abhängigkeit vom Verhältnis $p_{Z} / p$ zuerst in der Kreiszylinderschale eintreten [2]. Derartige Beulfälle weisen im Gegensatz zu axialgedrückten Kreiszylinderschalen und unverschieblich gelagerten Kugelschalen unter Radialdruck bereits bei elastischem Materialverhalten eine geringe Imperfektionsempfindlichkeit auf. Dabei ist die Änderung der Gauß'schen Krümmung am Übergang zwischen den Teilschalen zumindest für die analysierten Schalen von untergeordneter Bedeutung. 


\section{Kinetische Grenzlasten und Optimierung von Schalentragwerken}

Das Tragverhalten von Schalentragwerken unter kinetischen Belastungen ist durch verschiedene Phänomene charakterisiert. Mittels der Störenergie ist die Stabilität von Grundzuständen bei kurzzeitigen kinetischen Belastungen zu bewerten und können Schalentragwerke hinsichtlich der Imperfektionsempfindlichkeit optimiert werden.

\subsection{Kugelschale unter Radialdruck und Impulsbelastung}

Für eine stählerne Kugelschale unter statischem Radialdruck wird die Stabilität eines Gleichgewichtszustandes gegenüber einer kinetischen Störlast $p_{p}$ analysiert. Der Radialdruck bewirkt im Vorbeulbereich insbesondere für höhere Lastniveaus nichtlineares Tragverhalten, vgl. Bild 8.1. Für das Lastniveau $p / p_{c l}=0,67$ wird der Verzweigungspunkt $B$ ermittelt. Am Maximum der aufnehmbaren Belastung kann symmetrisches Durchschlagen einsetzen. Das Last-Verformungs-Diagramm verdeutlicht auch, dass die Imperfektionsempfindlichkeit eines Grundzustandes vom Lastniveau abhängig ist. Störlasten, die auf einen Grundzustand unterhalb des Verzweigungspunktes einwirken, können den Übergang des Systems in den Nachbeulbereich initiieren.
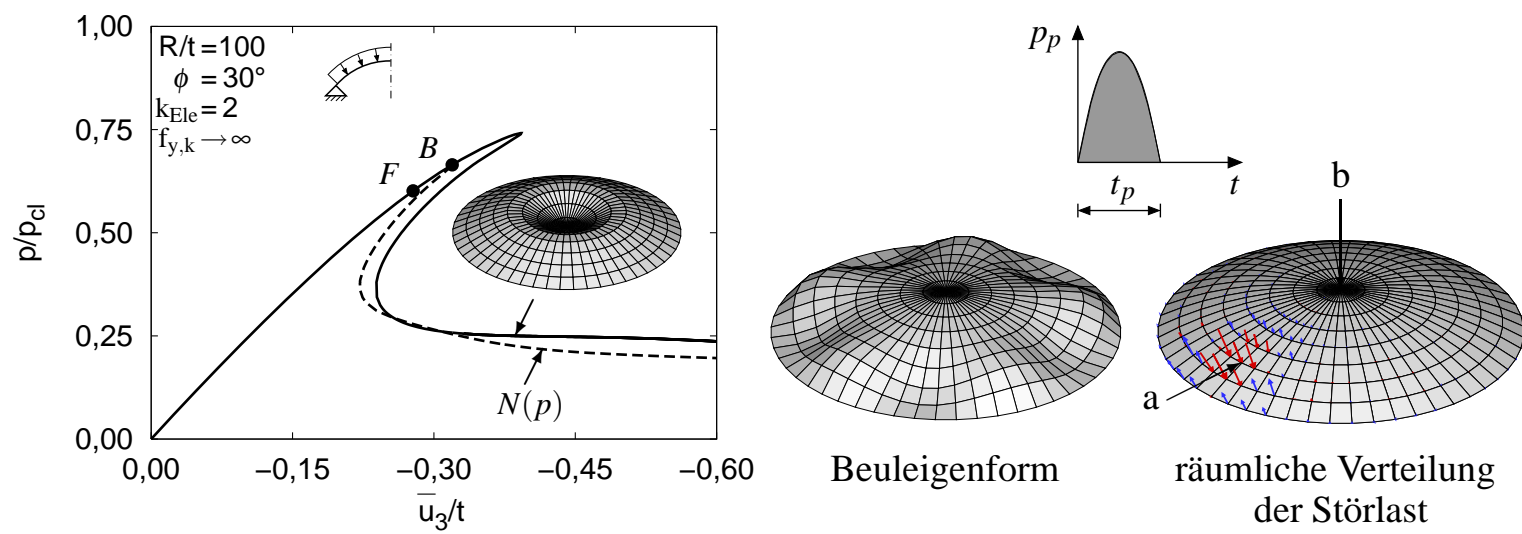

Bild 8.1: Last-Verformungs-Diagramm, Beuleigenform und Störlast

Zur Analyse der Stabilität des Grundzustandes $F$ auf dem Lastniveau $p / p_{c l}=0,60$ wird der kritische Zustand $N$ mittels Eigenwertproblem (4.8) und die räumliche Verteilung der Störlast gemäß Gl. (4.7) ermittelt. Die Störlast ist annähernd orthogonal zur Grundbelastung sowie lokal im Bereich des gelagerten Randes konzentriert und lässt eine im Vergleich zum Grundzustand und zur Beuleigenform unterschiedliche 
Systemantwort erwarten. Die zeitliche Verteilung der Störlast wird exemplarisch mit einer Sinus-Halbwelle der Dauer $t_{p}=1,2 \mathrm{~ms}$ beschrieben. Bild 8.2 zeigt die von Störlasten unterschiedlicher Intensität angeregten radialen Verschiebungen im Bereich der maximalen Störlastordinate und des Scheitelpunktes der Kugelschale.
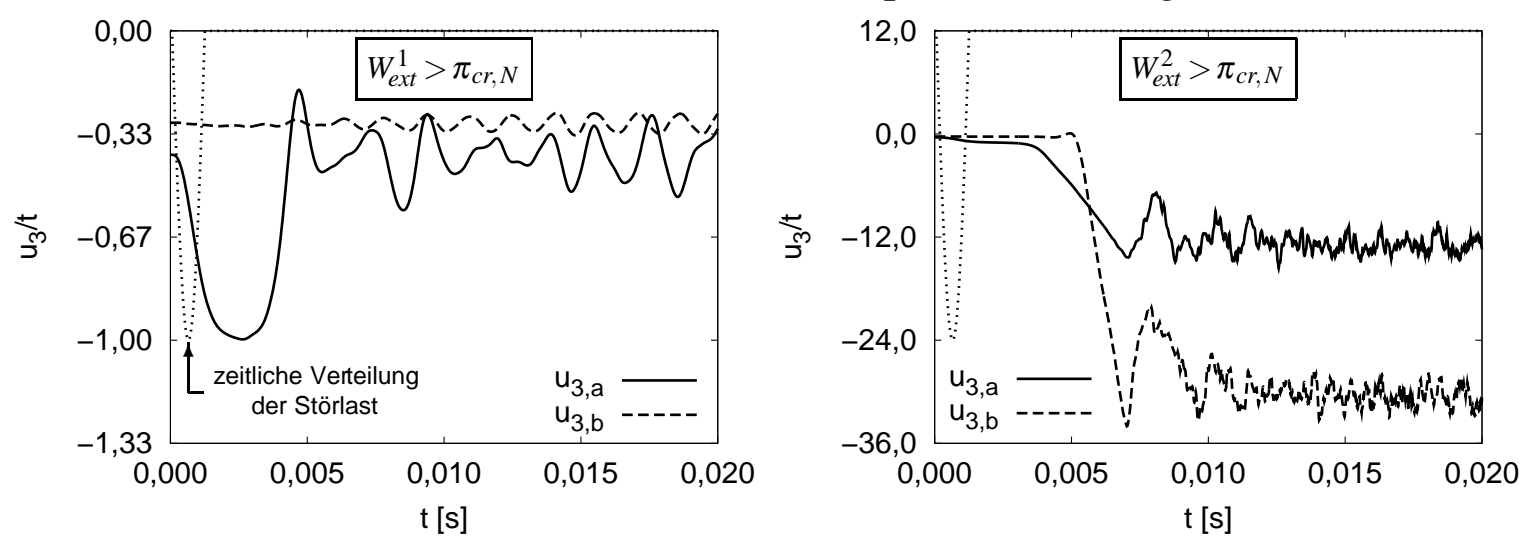

Bild 8.2: Verformungen infolge verschiedener Störlastintensitäten

Bei einer Störlast, welche die bezogene Energie $W_{e x t}^{1}=0,484 \%>0,443 \%=\pi_{c r, N}$ in das System einprägt, tritt die maximale Verschiebung im Bereich der Störlast und zeitlich geringfügig verzögert nach dem Einwirken der Störlast auf. Die Verschiebung ist etwa so groß wie die Wanddicke und nimmt aufgrund des Energieaustausches zwischen verschiedenen Schwingungsmoden schnell ab. Gleichzeitig breitet sich die Systembewegung in Breitenkreisrichtung aus. Der Grundzustand ist nicht empfindlich gegenüber der Störlast. Wird dagegen durch die Störlast die bezogene Energie $W_{\text {ext }}^{2}=0,485 \%$ in das System eingeprägt, vergrößert sich zunächst die Verschiebung im Bereich der Störlast und dann auch die Verschiebung des Scheitelpunktes deutlich. Die Systembewegung wird von einem Attraktor bzw. stabilen Gleichgewichtszustand im Nachbeulbereich angezogen. Die Kugelschale schlägt entsprechend experimenteller Erfahrungen [85] und Bild 8.3 innerhalb sehr kurzer Zeit unsymmetrisch durch.
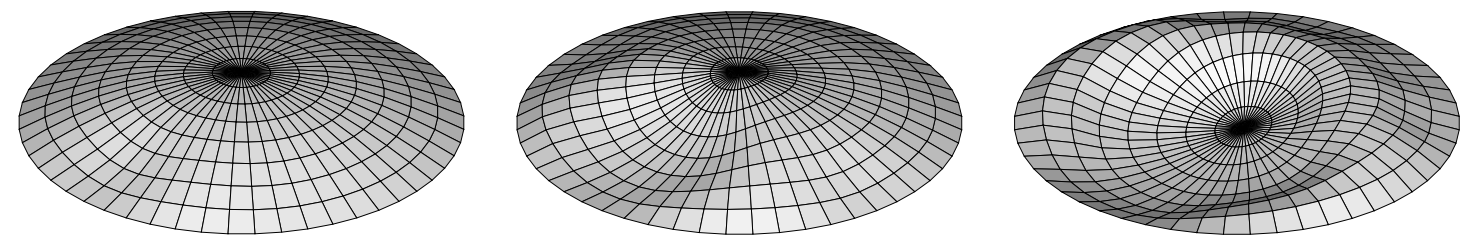

Bild 8.3: Kugelschale während des Beulvorganges ( $t=0,004 s, 0,005 s, 0,006 s)$

Da die Kugelschale bei der bezogenen eingeprägten Energie $W_{e x t}^{1}>\pi_{c r, N}$ nicht instabil wird, ist die Genauigkeit des Stabilitätsgrades (4.21) eingehender zu untersuchen.

\subsubsection{Genauigkeit des Stabilitätsgrades}

Die Definition des Stabilitätsgrades (4.21) lässt erwarten, dass dessen Genauigkeit von dem Lastniveau, den dynamischen Eigenschaften der Kugelschale und der Dis- 
kretisierung der Lagrange-Funktion (2.9) abhängig ist. Zur Bewertung des Einflusses des Lastniveaus werden mehrere Grundzustände einer kinetischen Störlast ausgesetzt. Die räumliche Verteilung der Störlast wird jeweils mittels Eigenwertproblem (4.8) und G1. (4.7) berechnet. Die zeitliche Verteilung der Störlast ist mit einer Sinus-Halbwelle beschrieben, deren Dauer jeweils der halben, zur ersten Schwingungseigenform gehörenden Periodendauer $T\left(\omega_{1}\right)$ entspricht.
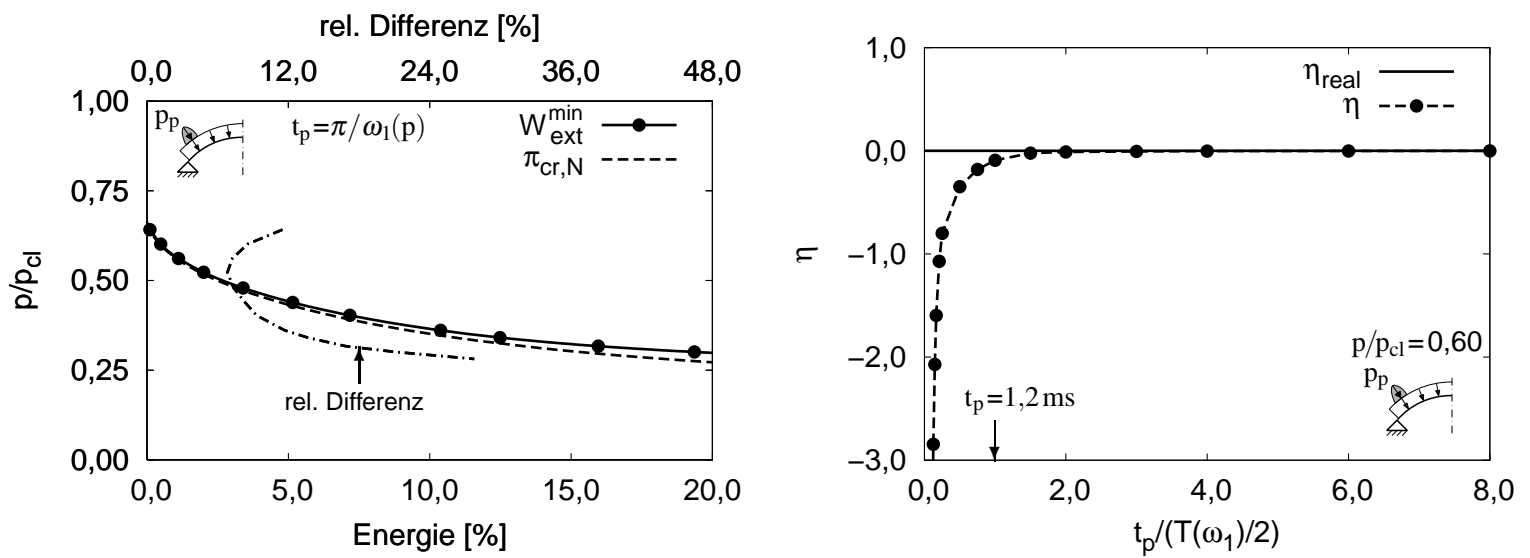

Bild 8.4: Genauigkeit des Stabilitätsgrades vs. Lastniveau und Dauer der Störung

Für verschiedene Lastniveaus zeigt Bild 8.4 links neben der bezogenen Störenergie die zum Initiieren von Instabilität einzuprägende Energie $W_{e x t}^{\min }$ auf. Diese Energie ist stets größer als die bezogene Störenergie. Somit beschreibt der Stabilitätsgrad $\eta$ unabhängig vom Lastniveau eine untere Grenze für die Stabilität eines Grundzustandes. Die relative Differenz zwischen bezogener Stör- und einzuprägender Energie beträgt für mittlere Lastniveaus etwa $10 \%$. Für Lastniveaus nahe dem Nachbeulminimum steigt die Differenz jedoch deutlich an. Die Differenz ist mit der Definition des Stabilitätsgrades zu begründen: Während der Ermittlung der Widerstandsfähigkeit eines Systems gegenüber Störungen nur ein Verformungsmode zugrunde liegt, werden von einer kinetischen Störlast sämtliche Schwingungsmoden des Systems, wenn auch mit unterschiedlicher Intensität, angeregt. Folglich ist zum Erreichen des kritischen Zustandes immer eine Energie in das System einzuprägen, die größer als die Störenergie ist. Die Differenz zwischen den Energien und somit die Genauigkeit des Stabilitätsgrades ist, wie Bild 8.4 rechts für einen Grundzustand verdeutlicht, auch von der Dauer der Störlast abhängig. Übersteigt die Dauer die halbe, zur ersten Schwingungseigenform gehörende Periodendauer, so repräsentiert der Stabilitätsgrad ein adäquates Kriterium zur Bewertung der Stabilität des Grundzustandes. Ansonsten wird dessen Stabilität, wie anhand des tatsächlichen Stabilitätsgrades $\eta_{\text {real }}$ eines indifferenten Systemzustandes erkennbar ist, teils sehr konservativ bewertet.

Die Diskretisierung der Lagrange-Funktion muss eine adäquate Beschreibung des Tragverhaltens ermöglichen und unter Berücksichtigung der räumlichen und zeitlichen Verteilung der Störlast erfolgen. Für die räumliche Diskretisierung sind die 
relevanten Beul- und Schwingungseigenformen maßgebend. Den Einfluss der räumlichen Diskretisierung auf verschiedene Systemkennwerte stellt Tabelle 8.1 heraus.

\begin{tabular}{|c|c|c|c|c|}
\hline \multicolumn{5}{|c|}{$R / t=100, t=10 \mathrm{~mm}, \rho=7.850 \mathrm{~kg} / \mathrm{m}^{3}, t_{p}=1,2 \mathrm{~ms}$} \\
\hline$k_{\text {Ele }}$ & $\begin{array}{c}\omega_{1} \\
{[\mathrm{rad} / \mathrm{s}]}\end{array}$ & $\begin{array}{c}\pi_{c r, N} \\
{[\%]}\end{array}$ & $\begin{array}{c}W_{\text {ext }}^{\text {min }} \\
{[\%]}\end{array}$ & $\begin{array}{c}\text { rel. Differenz } \\
{[\%]}\end{array}$ \\
\hline \hline 2 & $2.635,6$ & 0,443 & 0,485 & 8,660 \\
\hline 3 & $2.667,3$ & 0,413 & 0,449 & 8,018 \\
\hline 4 & $2.673,8$ & 0,412 & 0,447 & 7,830 \\
\hline
\end{tabular}

Tabelle 8.1: Systemkennwerte vs. Diskretisierung $\left(p / p_{c l}=0,60\right)$

Die Eigenkreisfrequenz $\omega_{1}$ ist nur geringfügig von den gewählten Elementierungsgraden abhängig. Dagegen konvergieren die bezogene Störenergie und die zum Initiieren von Instabilität einzuprägende Energie $W_{e x t}^{\min }$ etwas langsamer gegen den genauen Wert. Dies gilt auch für die relative Differenz zwischen beiden Energien und somit für die Genauigkeit des Stabilitätsgrades. Ferner erlaubt die im Fall statischer Störungen übliche Berechnung an Teilsystemen bei kinetischen Störlasten nur für ausgewählte Systeme eine adäquate Bewertung der Stabilität. Die zeitliche Diskretisierung erfolgt unter Berücksichtigung der Dauer der Störlast und der abzubildenden Schwingungsmoden mit einer Zeitschrittweite von $T\left(\omega_{1}\right) / 40$ bis $T\left(\omega_{1}\right) / 80$ [114]. Insofern beeinflusst die zeitliche Diskretisierung die Ergebnisse nicht wesentlich.

Zusammenfassend ist festzuhalten, dass die Genauigkeit des Stabilitätsgrades primär davon abhängig ist, wie stark die Störlast bis zum Erreichen des kritischen Zustandes Schwingungsmoden anregt, die sich von den Verformungen $\Delta \mathbf{u}_{c r, N}$ unterscheiden. Daher ist die Stabilität eines Grundzustandes insbesondere im Fall kinetischer Belastungen, deren Dauer nicht wesentlich kleiner ist als die halbe, zur ersten Schwingungseigenform gehörende Periodendauer, adäquat mit dem Stabilitätsgrad zu bewerten. Ferner ist zu erkennen, dass der Stabilitätsgrad zwei wesentliche Merkmale einer Bewegung, nämlich deren Energie und Stabilität, miteinander verknüpft.

\subsubsection{Räumliche und zeitliche Verteilung der Störlast}

Anhand des Stabilitätsgrades (4.21) wird zunächst der Einfluss der räumlichen Verteilung der Störlast auf die Stabilität des Grundzustandes $F$ aufgezeigt. Dabei werden drei zur bisherigen Störlast räumlich unterschiedlich verteilte Störlasten berücksichtigt. Deren zeitliche Verteilung folgt einer Sinus-Halbwelle der Dauer $t_{p}=1,2 \mathrm{~ms}$. Die Stabilität des Grundzustandes wird jeweils für eine Dauer von $32 t_{p}$ bewertet.

Die erste Störlast resultiert auch aus Eigenwertproblem (4.8), ist annähernd orthogo- 

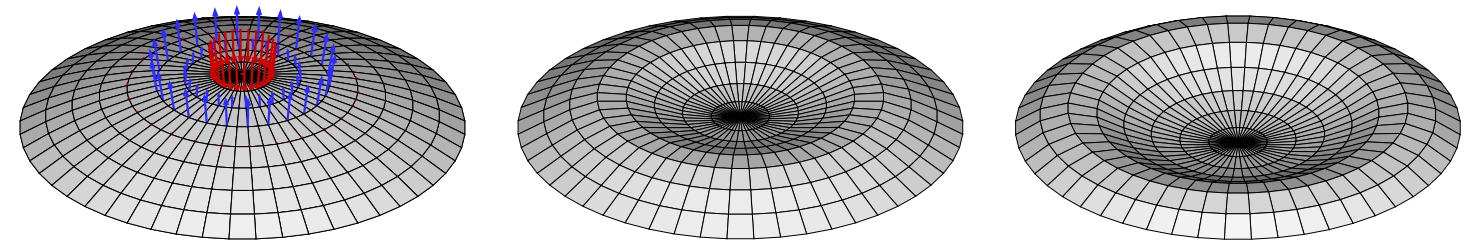

Bild 8.5: Symmetrische Störlast und Beulvorgang $(t=0,0080 s, 0,0085 s)$

nal zur Grundbelastung und, wie in Bild 8.5 angedeutet, rotationssymmetrisch sowie im Bereich des Scheitelpunktes der Kugelschale konzentriert. Der räumlichen Verteilung der Störlast ist ein kritischer Zustand $M$ zugehörig. Ausgehend vom Grundzustand wird in Richtung des Zustandes $M$ ein kritischer Zustand $N$ ermittelt, der durch die bezogene Störenergie $\pi_{c r, N}=3,157 \%$ charakterisiert ist. Die Störlast regt die Kugelschale zu rotationssymmetrischen Schwingungen an. Überschreitet die bezogene eingeprägte Energie 3,196\%, schlägt die Kugelschale rotationssymmetrisch durch. Die relative Differenz zwischen bezogener Stör- und eingeprägter Energie beträgt ca. 1,2\% und lässt erkennen, dass höherfrequente Schwingungsmoden in Meridianrichtung kaum auftreten. Für die Bewertung der Stabilität des Grundzustandes ist primär entscheidend, ob Änderungen der räumlichen Verteilung der Störlast auszuschließen sind. Ist dies nicht der Fall, so ist der zur unsymmetrischen Störlast zugehörige kritische Zustand $N$ der Bewertung der Stabilität des Grundzustandes zugrunde zu legen.

Die in Bild 8.6 dargestellte nicht rotationssymmetrische Störlast ist affin zur ersten Beuleigenform der Kugelschale und orthogonal zur Grundbelastung.
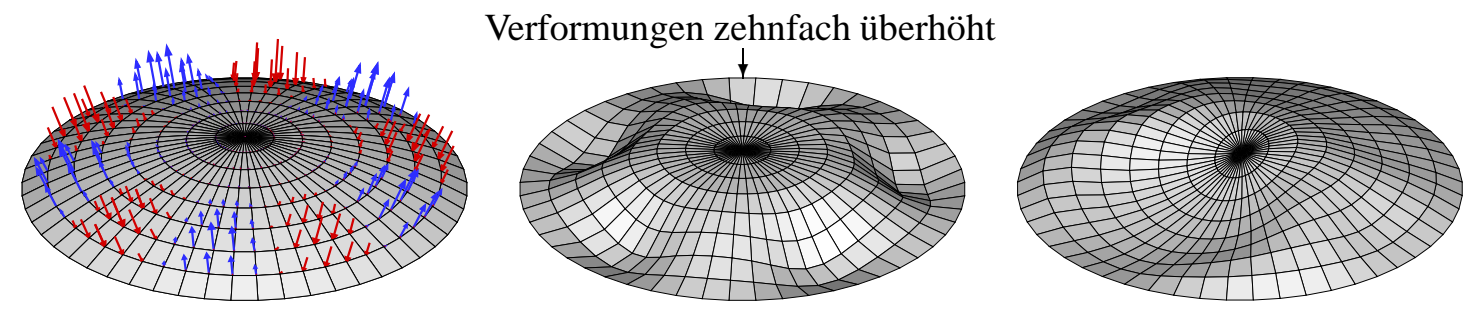

Bild 8.6: Beuleigenformaffine Störlast und Beulvorgang $(t=0,002 s, 0,014 s)$

Die Störlast regt für den Fall $W_{\text {ext }}<W_{\text {ext }}^{\text {min }}$ das System zu Schwingungen an, die der ersten Beuleigenform ähnlich sind. Infolge des Energieaustausches zwischen verschiedenen Schwingungsmoden nähert sich die Systembewegung einem kritischen $\mathrm{Zu}$ stand. Für die bezogene eingeprägte Energie $W_{e x t}^{\text {min }}=1,534 \%$ schlägt die Kugelschale wie bei der Störlast gemäß Bild 8.1 unsymmetrisch durch. Die Energie $W_{e x t}^{\text {min }}$ ändert sich bei einer zur zweiten Beuleigenform affinen Störlast nur geringfügig.

Eine zur Grundbelastung affine Störlast regt die Kugelschale zu rotationssymmetrischen Schwingungen an. Überschreitet die bezogene eingeprägte Energie 5,059\%, schlägt die Kugelschale rotationssymmetrisch durch. Diese relativ große Energie ist damit zu begründen, dass das Maximum des primären Last-Verformungs-Pfades einen 
kritischen Zustand repräsentiert, vgl. Bild 8.1. Der kritische Zustand resultiert nicht aus Eigenwertproblem(4.8) und ist nur dann der maßgebende kritische Zustand, wenn Änderungen der räumlichen Verteilung der Störlast und die Annäherung der Systembewegung an andere kritische Zustände auszuschließen sind. Für diesen Fall wird die für die Bewertung der Stabilität relevante Differenz an bezogener Formänderungsenergie mit dem zweiten und dritten Term der Gl. (4.14) zu 3,468\% ermittelt.

Für die vier berücksichtigten Störlasten ist in Bild 8.7 das Verhältnis der bezogenen eingeprägten Energie $W_{e x t}^{\text {min }}$ zur bezogenen Störenergie $\pi_{c r, N}=0,443 \%$ aufgezeigt.

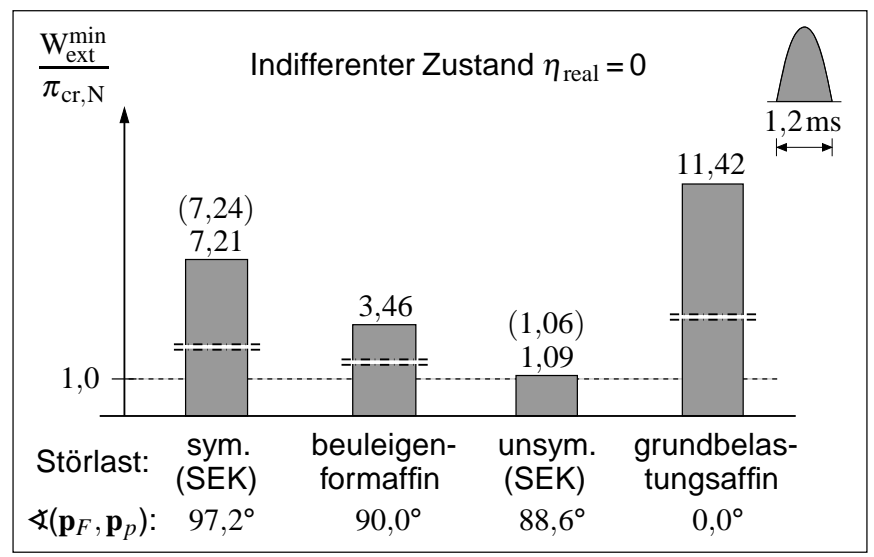

Bild 8.7: Verhältnis der Energien vs. Störlast $\left(p / p_{c l}=0,60\right)$

Bei den rotationssymmetrischen Störlasten ist am meisten Energie in das System einzuprägen, um Instabilität zu bewirken. Derartige Störlasten sind somit zur Bewertung der Stabilität eines Grundzustandes nur bedingt geeignet. Ähnliches gilt für die beuleigenformaffine Störlast. Dahingegen ermöglicht die mit dem Störenergie-Konzept (SEK) ermittelte unsymmetrische Störlast eine präzise Bewertung der Stabilität. Dies ist, wie in Bild 8.8 links für die Störlasten gemäß Störenergie-Konzept angedeutet, mit den unterschiedlichen kritischen Zuständen zu begründen. Werden die Störlasten gemäß Störenergie-Konzept durch eine im Scheitelpunkt der Kugelschale bzw. im Knoten $a$ angreifende Einzellast ersetzt, so ändert sich das Tragverhalten der Kugelschale nur unwesentlich. Dies bestätigen die Klammerwerte in Bild 8.7. Wird dagegen die grundbelastungsaffine Störlast um eine im Knoten $a$ angreifende Einzellast gleicher Lastordinate ergänzt, so nimmt die Energie $W_{e x t}^{\min }$ um 57,6\% ab. Ähnliches ist für die rotationssymmetrische Störlast gemäß Störenergie-Konzept zu erwarten. Insofern verdeutlichen die Ergebnisse die Bedeutung des Störenergie-Konzeptes sowohl für die Ermittlung ungünstiger Störlasten als auch für die Beschreibung der Widerstandsfähigkeit von Schalentragwerken gegenüber Störungen. Dies gilt auch für den Fall mehrerer nacheinander einwirkender Störlasten.

Die zeitliche Verteilung einer Störlast ist durch Verlaufsfunktion und Dauer charakterisiert. Den Einfluss der Verlaufsfunktion auf die zum Initiieren von Instabilität ein- 
zuprägende Energie stellt Tabelle 8.2 für die unsymmetrische Störlast gemäß Störenergie-Konzept heraus. Die Dauer der Störlast beträgt jeweils 1,2 ms.

\begin{tabular}{|c|c|c|c|c|c|}
\hline \multicolumn{5}{|c|}{$R / t=100, t=10 \mathrm{~mm}, k_{\text {Ele }}=2, \rho=7.850 \mathrm{~kg} / \mathrm{m}^{3}, \pi_{c r, N}=0,443 \%$} \\
\hline $\mathbf{p}_{p}(t)$ & $\begin{array}{c}W_{\text {ext }}^{\text {min }}[\%] \\
{[\%]}\end{array}$ & $\begin{array}{c}\text { rel. Differenz } \\
{[\%]}\end{array}$ & $\mathbf{p}_{p}(t)$ & $\begin{array}{c}W_{\text {ext }}^{\text {min }} \\
{[\%]}\end{array}$ & $\begin{array}{c}\text { rel. Differenz } \\
{[\%]}\end{array}$ \\
\hline \hline$\Lambda$ & 0,493 & 10,142 & $\square$ & 0,487 & 9,035 \\
\hline$\Lambda$ & 0,509 & 12,967 & $\cap$ & 0,485 & 8,660 \\
\hline
\end{tabular}

Tabelle 8.2: Einzuprägende Energie vs. Verlaufsfunktion der Störlast $\left(p / p_{c l}=0,60\right)$

Die einzuprägende Energie variiert für die verschiedenen Verlaufsfunktionen nur geringfügig. Für die sinusförmige Verlaufsfunktion ist die einzuprägende Energie am geringsten und folglich die Genauigkeit des Stabilitätsgrades am größten. Die Dauer der Störlast beeinflusst neben der Genauigkeit des Stabilitätsgrades auch den zeitlichen Verlauf der eingeprägten Energie, vgl. Bild 8.8 rechts. Die maximale Steigung
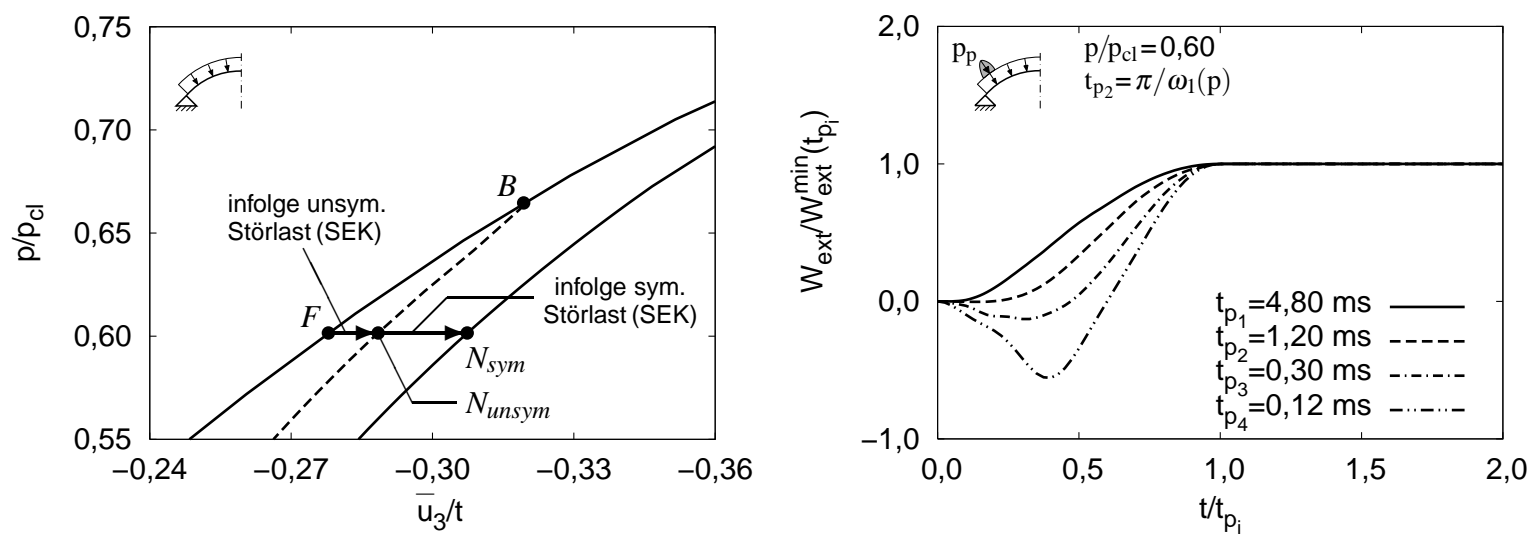

Bild 8.8: Detail des Last-Verformungs-Pfades und Verlauf der eingeprägten Energie

im Verlauf der eingeprägten Energie tritt, relativ zur jeweiligen Dauer der Störlast, mit abnehmender Dauer später auf. Nach dem Einwirken der Störlast besitzt das System mehr Energie. Ist die Dauer der Störlast kleiner als die halbe, zur ersten Schwingungseigenform gehörende Periodendauer, so weist die eingeprägte Energie temporär ein negatives Minimum auf. Das Minimum wird mit abnehmender Dauer der Störlast betragsmäßig größer und tritt, wie Bild 8.9 für den Knoten $a$ verdeutlicht, etwa zeitgleich mit dem Extremum der Massenbeschleunigung auf. Die Massenbeschleunigung wird ebenfalls mit abnehmender Dauer der Störlast betragsmäßig merklich größer. Gleichzeitig verringern sich während des Einwirkens der Störlast die Verformungen des Systems. Eine Bewegung des Systems entgegen den Lastkomponenten der Störlast ist aber nur für wenige Knoten und dann nur in sehr geringem Maß festzustellen. Somit verursachen die Lastkomponenten nicht das temporäre Minimum an eingeprägter Energie. Das Minimum ist, wie in Bild 8.10 für zwei Störlasten un- 

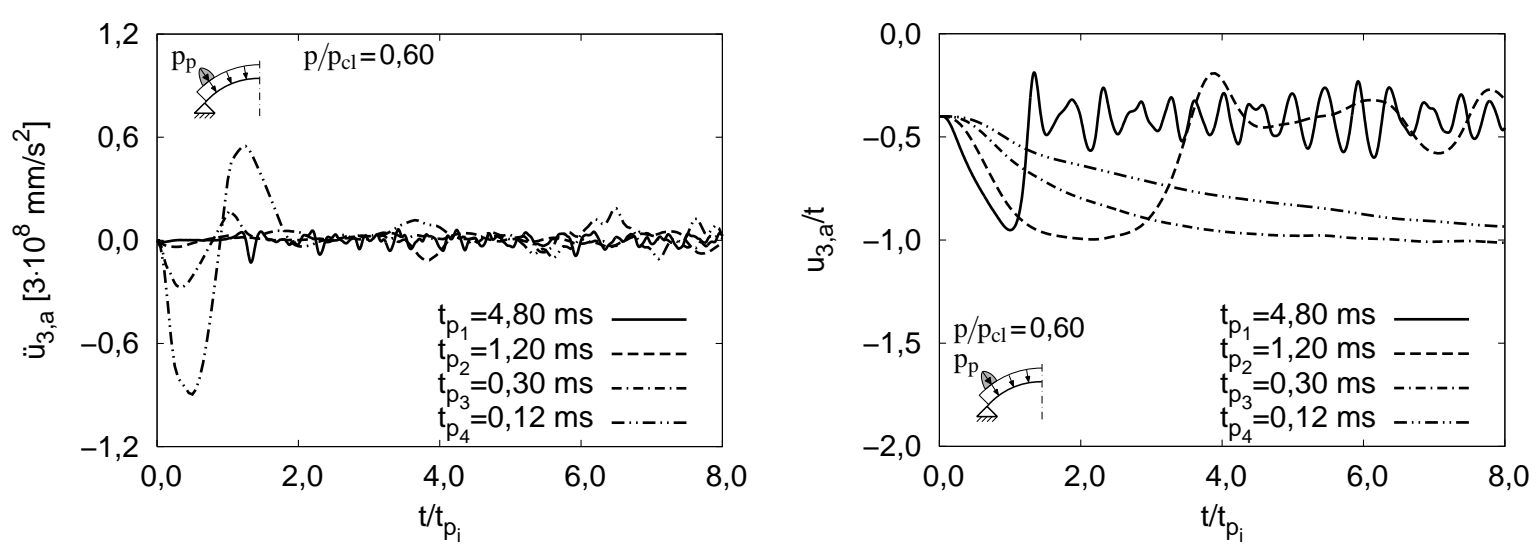

Bild 8.9: Beschleunigung und Verschiebung des Knotens $a$ vs. Dauer der Störlast

terschiedlicher Dauer aufgezeigt, mit den negativen und positiven Störverzerrungen $\alpha_{\alpha \beta, p}$ und der zeitlichen Änderung der Membrankräfte zu begründen.
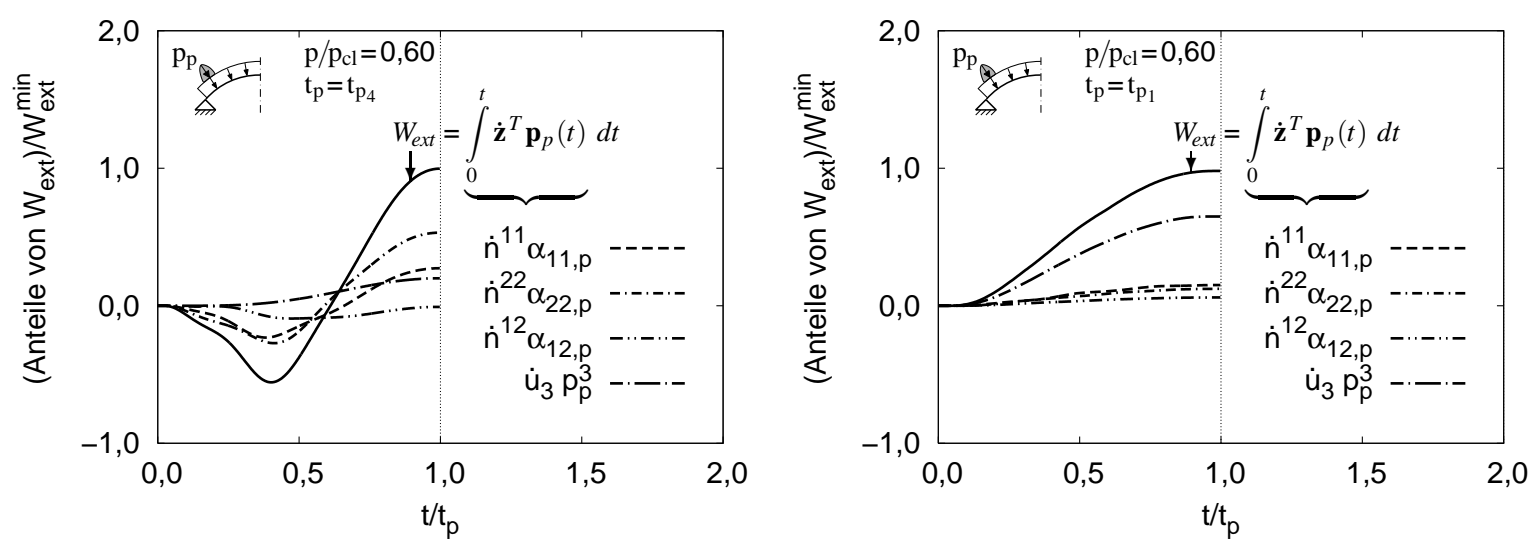

Bild 8.10: Anteile der eingeprägten Energie vs. Dauer der Störlast

Die Änderung der Membrankräfte ist räumlich begrenzt und primär von der Dauer der Störlast abhängig. So ist für die Dauer $t_{p}=t_{p_{1}}$ die Wirkung der Störverzerrungen im Vergleich zur Wirkung der Lastkomponenten der Störlast gering. Die von den Lastkomponenten $p_{p}^{1}$ und $p_{p}^{2}$ eingeprägte Energie ist indes sehr klein. Obwohl das Minimum an eingeprägter Energie im vorliegenden Fall aus den nur in der gemischten Formulierung vorhandenen Störverzerrungen resultiert, ist ein entsprechendes Minimum im Rahmen einer Weggrößenformulierung nicht auszuschließen.

Für einige Störlasten, wie der grundbelastungsaffinen Störlast, ist während deren Einwirken temporär auch ein Maximum an eingeprägter Energie festzustellen. Die nachfolgende Verringerung der Energie ist mit der Bewegung des Systems entgegen der Störlast zu begründen und somit von deren zeitlicher Verteilung abhängig. Folglich wirkt die Störlast teilweise sogar stabilisierend. Solange aber die Störlast auf das System einwirkt und ein kritischer Zustand noch nicht erreicht wurde, ist keine endgültige Aussage zur Stabilität des Systems möglich. Abschließend ist zu betonen, dass die Ergebnisse zur Genauigkeit des Stabilitätsgrades sowie zum Einfluss der räumli- 
chen und zeitlichen Verteilung der Störlast auf die Stabilität eines Systems aufzeigen, inwiefern sich die erste und die zweite Methode nach Ljapunow ergänzen.

Grenzlasten. Die kinetische Grenzlast eines Schalentragwerkes ist bezüglich eines definierten Grundzustandes zu ermitteln. Wird für die kinetische und die statische Grenzlast ein energetisch äquivalenter Einfluss der ungünstigsten Störung vorausgesetzt, so ist gemäß Bild 8.11 links die Belastung im Grundzustand, dem die kinetische Grenzlast zugehörig ist, größer als die statische Grenzlast, vgl. Bild 4.4.
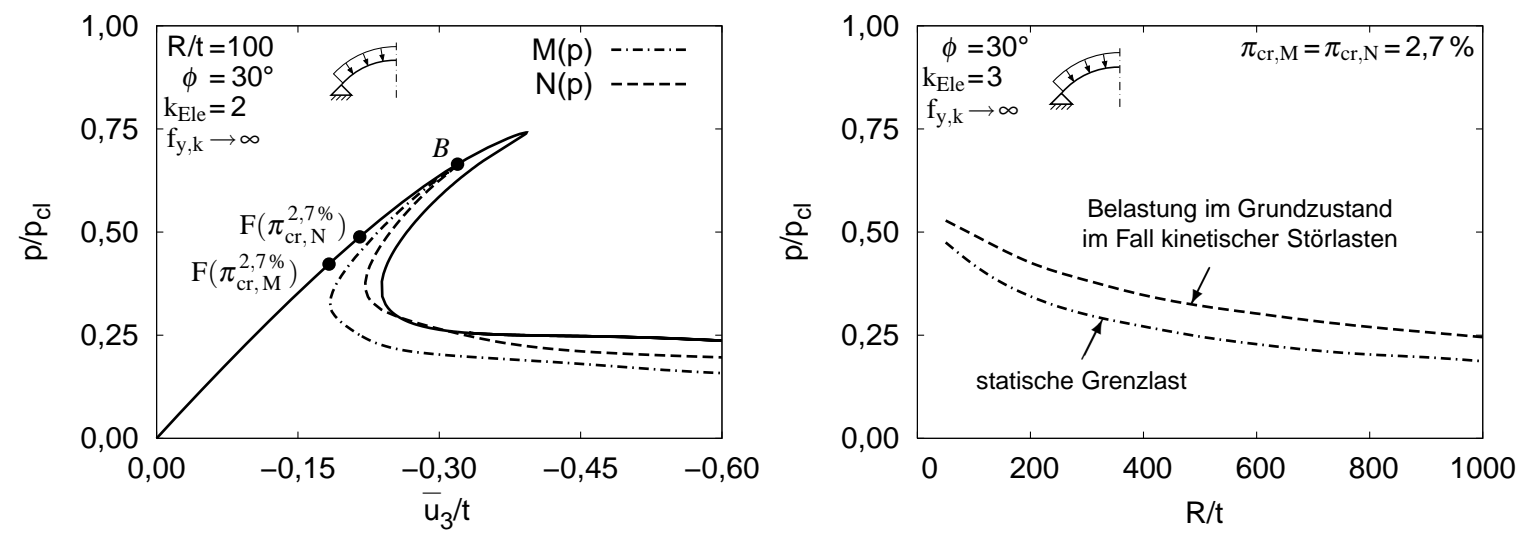

Bild 8.11: Statische und kinetische Grenzlasten gemäß Störenergie-Konzept

Die Differenz zwischen beiden Lastniveaus variiert, wie Bild 8.11 rechts aufzeigt, in Abhängigkeit von der Schlankheit um bis zu 33\%. Eine Beschreibung der Grundzustände, denen die kinetischen Grenzlasten zugehören, ist in Anlehnung an DAStRichtlinie 013, vgl. Bild 5.3, zwar denkbar, erscheint aber nicht sinnvoll, da die kinetische Grenzlast häufig bezüglich verschiedener Grundzustände zu ermitteln ist.

\subsubsection{Analyse eines Beulvorganges}

Der Beulvorgang eines Schalentragwerkes ist wie dessen Imperfektionsempfindlichkeit von mehreren Parametern abhängig und wird nachfolgend für eine Kugelschale unter Radialdruck und unsymmetrischer Störlast gemäß Gl. (4.7) bewertet. Dabei werden für eine Grundbelastung die der Systembewegung inhärenten Energien, der Zeitpunkt der Identifikation von Instabilität und die am Beulvorgang primär beteiligten Moden untersucht. Wesentliche Systemparameter sind Bild $8.1 \mathrm{zu}$ entnehmen.

Für zwei Störlastintensitäten ist in Bild 8.12 links neben dem zeitlichen Verlauf der eingeprägten Energie auch der zeitliche Verlauf der kinetischen Energie $T$ dargestellt, wobei der obere Index die Störlastintensität charakterisiert. Während des Einwirkens der Störlast erreicht die kinetische Energie ein Maximum. Unmittelbar nach dem Einwirken der Störlast ist primär Formänderungsenergie im System vorhanden. Die Systembewegung befindet sich in der Nähe des kritischen Zustandes. Je geringer dabei der Abstand zum kritischen Zustand, desto länger verbleibt die Systembewe- 

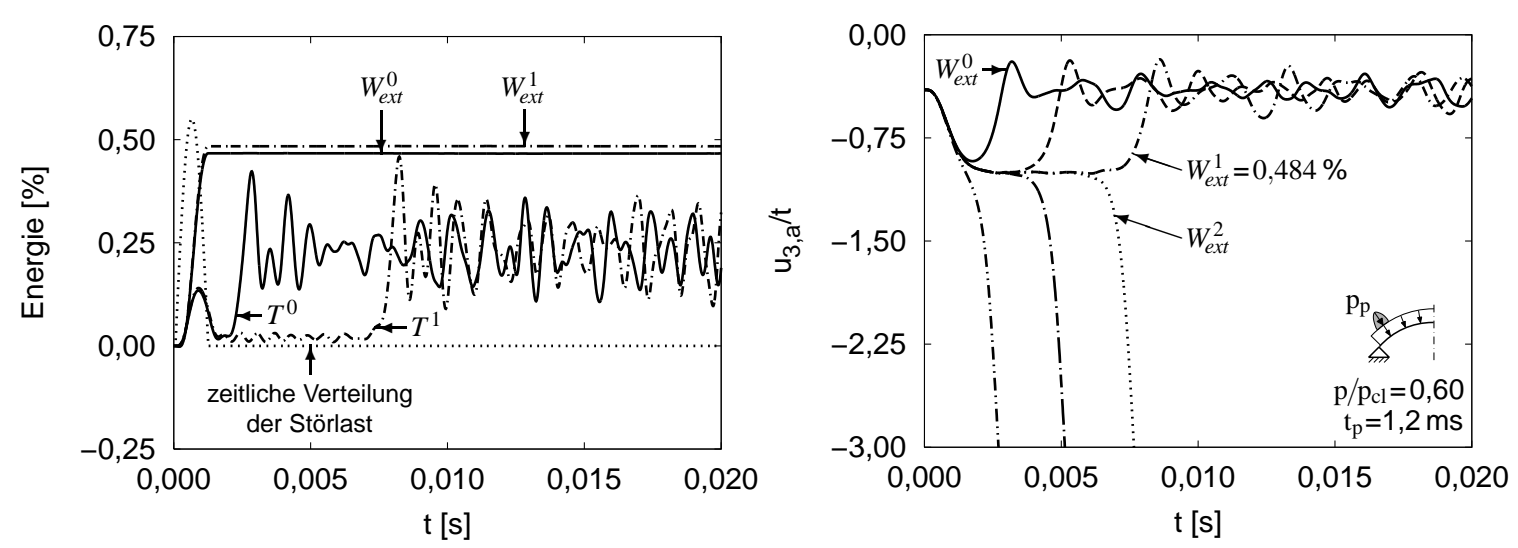

Bild 8.12: Zeitlicher Verlauf der Energien und einer charakteristischen Weggröße

gung in dessen Nähe. Anschließend findet ein ständiger Austausch von kinetischer und Formänderungsenergie statt. Infolge der Anregung verschiedener Schwingungsmoden kommt die stabile Systembewegung nicht mehr in die Nähe des kritischen Zustandes. Ferner verzögert ein fortdauernder Verbleib der Systembewegung in der Nähe des kritischen Zustandes die Identifikation von Instabilität mittels LjapunowExponenten oder einer charakteristischen Weggröße. Außerdem wird die Existenz einer Separatrix bzw. kritischen Trajektorie ersichtlich, was in Bild 8.12 rechts mit dem zeitlichen Verlauf der radialen Verschiebung des Knotens $a$, vgl. Bild 8.1, infolge der eingeprägten Energie $W_{e x t}^{2}$ verdeutlicht werden soll. Diese Energie ist um ca. $1,1 \cdot 10^{-5} \%$ größer als die Energie $W_{e x t}^{1}$, für welche die Systembewegung stabil ist.

Die Bewertung des Anteiles verschiedener Moden am Beulvorgang erfolgt anhand der Affinität $\alpha_{i}(t)$ zwischen den Verformungskomponenten des aktuellen Zustandsvektors $\Delta \mathbf{z}(\mathrm{t})=\mathbf{z}(\mathrm{t})-\mathbf{z}_{F}$ und denen der folgenden Zustandsvektoren:

- $\mathbf{z}_{1}: \quad$ inkrementelle Zustandsgrößen $\Delta \mathbf{z}_{c r, N_{\text {unsym }}}$ $\rightarrow \alpha_{1}(t)$

- $\mathbf{z}_{2}$ : inkrementelle Zustandsgrößen $\Delta \mathbf{z}_{c r, N_{s y m}}$ $\rightarrow \alpha_{2}(t)$

- $\mathbf{z}_{3}: \quad \mathbf{z}(t=6,990 \mathrm{~ms})$ der durchgeschlagenen Kugelschale $\rightarrow \alpha_{3}(t)$,

- $\mathbf{z}_{4}:$ erste Schwingungseigenform (fünfwellig, vgl. Bild 8.1) $\rightarrow \alpha_{4}(t)$.

Die Systemantwort auf die unsymmetrische Störlast ist zwar von deren Intensität abhängig, aber Bild 8.13 zufolge allgemein durch zwei Bereiche gekennzeichnet.

Innerhalb des ersten Bereiches nähert sich die Affinität $\alpha_{1}$ schnell dem Wert eins und bleibt auch einige Zeit nach dem Einwirken der Störlast nahezu unverändert. Diese Zeitspanne ist ein Indikator für die Annäherung der Systembewegung an den kritischen Zustand und der damit einhergehenden Verringerung der elastischen Rückstellkräfte des Systems. Im zweiten Bereich nimmt bei einer stabilen Systembewegung die Affinität $\alpha_{1} \mathrm{ab}$, die anderen Affinitäten hingegen nehmen zu. Die ungedämpfte Systembewegung breitet sich in Breitenkreisrichtung aus. Wird dagegen das System 

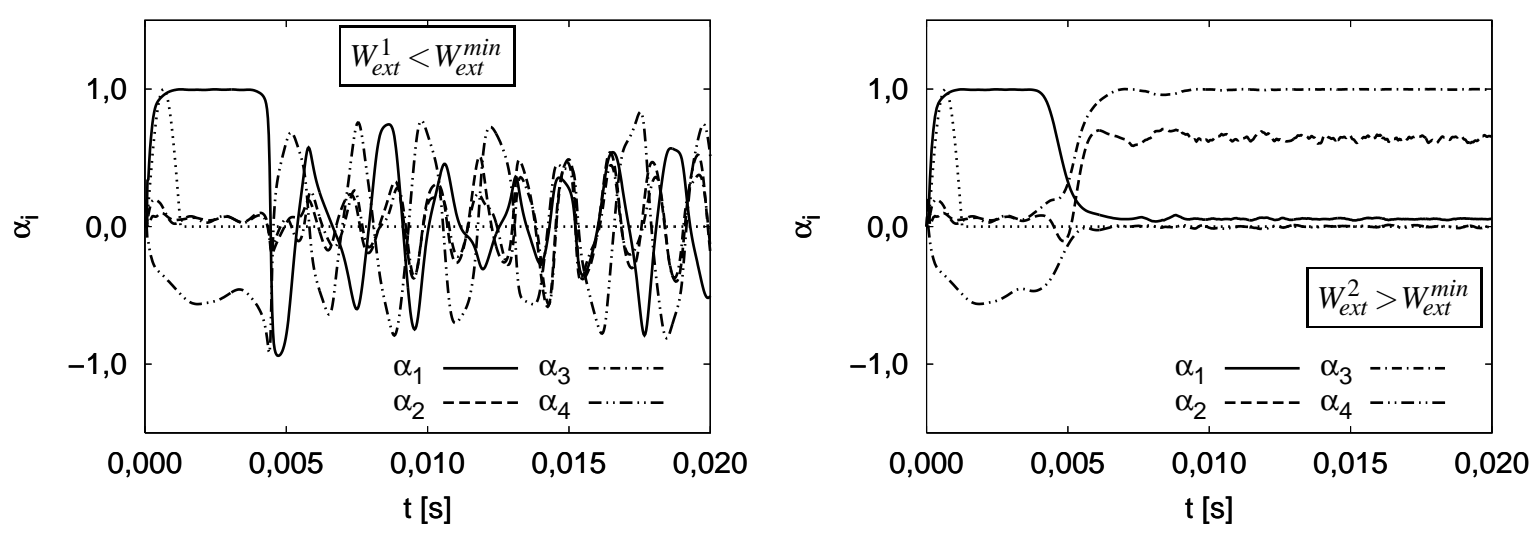

Bild 8.13: Verformungsanteile bei Systemstabilität und -instabilität

infolge der Störlast instabil, so dominieren im Nachbeulbereich primär rotationssymmetrische Zustände das Systemverhalten. Dabei bestätigen die geringen Affinitäten $\alpha_{1}$ und $\alpha_{4}$ die Existenz höherfrequenter Schwingungsmoden. Diese können physikalisch, aber auch numerisch bedingt sein. Letzteres wäre insbesondere auf das verwendete Zeitintegrationsverfahren [99], aber auch auf die Beschreibung der Kinematik des Schalenkontinuums im Sinne einer Theorie mäßiger Rotationen zurückzuführen.

\subsection{Kreiszylinderschale unter Axiallast und Impulsbelastung}

Für eine axialgedrückte Kreiszylinderschale mit der Schlankheit $r / t=100$ und dem Längen-Radius-Verhältnis $l / r=2$ wird die Stabilität des Grundzustandes auf dem Lastniveau $p / p_{c l}=0,75$ gegenüber verschiedenen kinetischen Störlasten untersucht. Elastizitätsmodul und Querdehnzahl entsprechen den in Abschnitt 8.1 verwendeten und in Abschnitt 5.1 angegebenen Werten. Die Dichte $\rho$ beträgt $7.850 \mathrm{~kg} / \mathrm{m}^{3}$. Die Kreiszylinderschale ist an beiden Rändern gelenkig gelagert, wobei Randverschiebungen nur am belasteten Rand und lediglich in Meridianrichtung möglich sind. Für den definierten Grundzustand und den Elementierungsgrad $k_{E l e}=1$ wird die bezogene Störenergie $\pi_{c r, N} \mathrm{zu} 0,514 \%$ ermittelt. Der zeitlichen Verteilung der Störlasten liegt eine Sinus-Halbwelle der Dauer $t_{p}=\pi / \omega_{1}=6,92 \mathrm{~ms}$ zugrunde.

Die räumliche Verteilung der ersten Störlast wird mittels Eigenwertproblem(4.8) und Gl. (4.7) ermittelt. Die Störlast ist, wie in Bild 8.14 angedeutet, im Bereich des belasteten Randes konzentriert und orthogonal zur Grundbelastung. Die Störlast initiiert eine Systembewegung im Bereich des belasteten Randes. Erreicht die Bewegung nicht unmittelbar nach dem Einwirken der Störlast den kritischen Zustand $N$, so breitet sich die Bewegung in Meridian- und Umfangsrichtung aus. Die zugehörigen Verformungen $\Delta \mathbf{z}(\mathrm{t})=\mathbf{z}(\mathrm{t})-\mathbf{z}_{F}$ sind hundertfach überhöht dargestellt. Wird jedoch von der Störlast die bezogene Energie $W_{e x t}^{1}=0,558 \%$ in das System eingeprägt, so tritt Instabilität lokal im Bereich des belasteten Randes ein, vgl. Bild 8.15 links. 

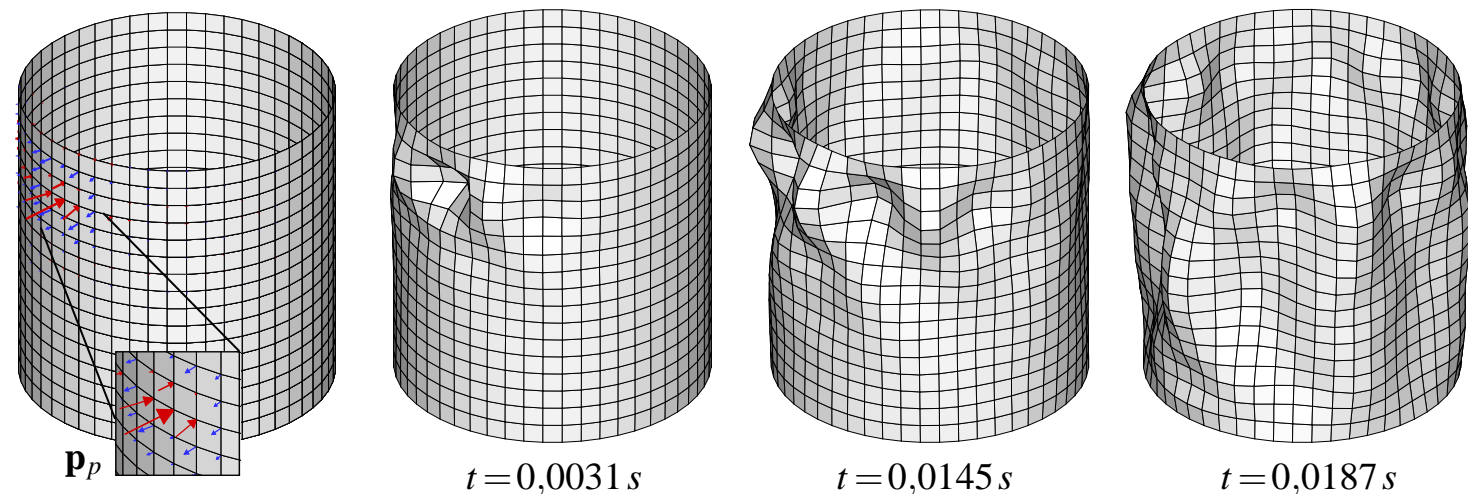

Bild 8.14: Störlast gemäß Störenergie-Konzept und stabile Systembewegung

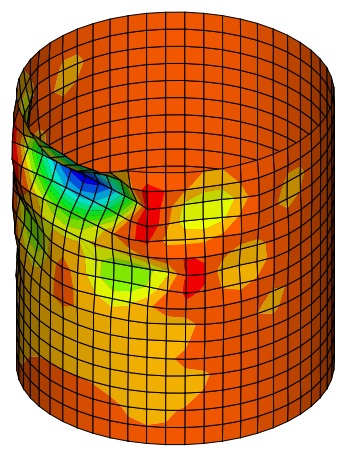

$t=0,0149 \mathrm{~s}$

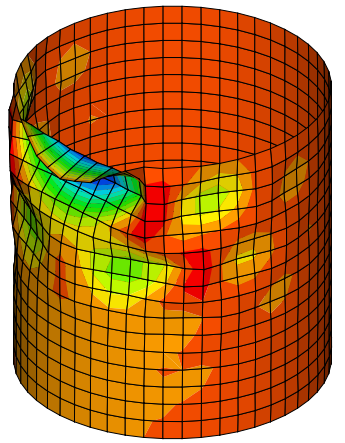

$t=0,0152 \mathrm{~s}$

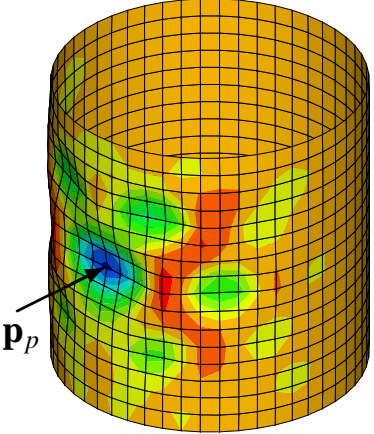

$t=0,0145 \mathrm{~s}$

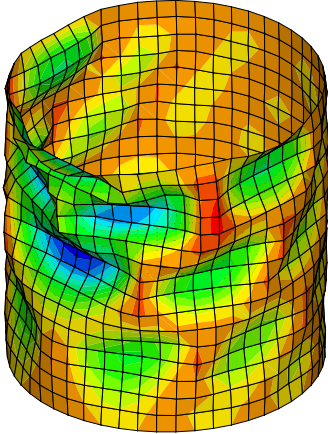

$t=0,0173 \mathrm{~s}$

Bild 8.15: Beulvorgang der Kreiszylinderschale vs. Störlast

Bei einer Störlast, die nur aus einer Einzellast besteht und auf halber Länge der Kreiszylinderschale radial nach innen wirkt, wird das System für die bezogene Energie $W_{e x t}^{2}=1,175 \%$ instabil. In diesem Fall beträgt die Amplitude der Störlast 0,041 \% der Grundbelastung. Der Beulvorgang ist im Gegensatz zu dem infolge der ersten Störlast, und wie in Bild 8.15 rechts dargestellt, durch eine größere Ausdehnung sowohl in Meridian- als auch in Umfangsrichtung gekennzeichnet. Dabei ist bereichsweise ein rautenförmiges Beulmuster erkennbar. Wird der Angriffspunkt der Störlast um ein Viertel der Länge der Kreiszylinderschale in Meridianrichtung nach unten verschoben, so ist die bezogene Energie $W_{e x t}^{3}=1,661 \%$ erforderlich, um Instabilität zu bewirken. Den zugehörigen Beulvorgang zeigt Bild 8.16 auf.

Für den Beulvorgang sind neben der Entwicklung des Beulmusters die Verwölbung der Kreiszylinderschale am belasteten Rand und deren Stauchung in Meridianrichtung charakteristisch. Mit einer feineren räumlichen Diskretisierung ist der Beulvorgang zwar genauer abzubilden, die Energie $W_{\text {ext }}^{\text {min }}$ wird sich dabei aber nur unwesentlich ändern. Zur Erfassung des gesamten Beulvorganges wäre der bei großen Deformationen mögliche Kontakt zwischen Wandabschnitten zu berücksichtigen [56].

Eine grundbelastungsaffine Störlast regt das System für $W_{e x t}<W_{e x t}^{\min }$ zu rotationssymmetrischen Schwingungen an. Übersteigt die bezogene eingeprägte Energie ca. 30,4\%, 

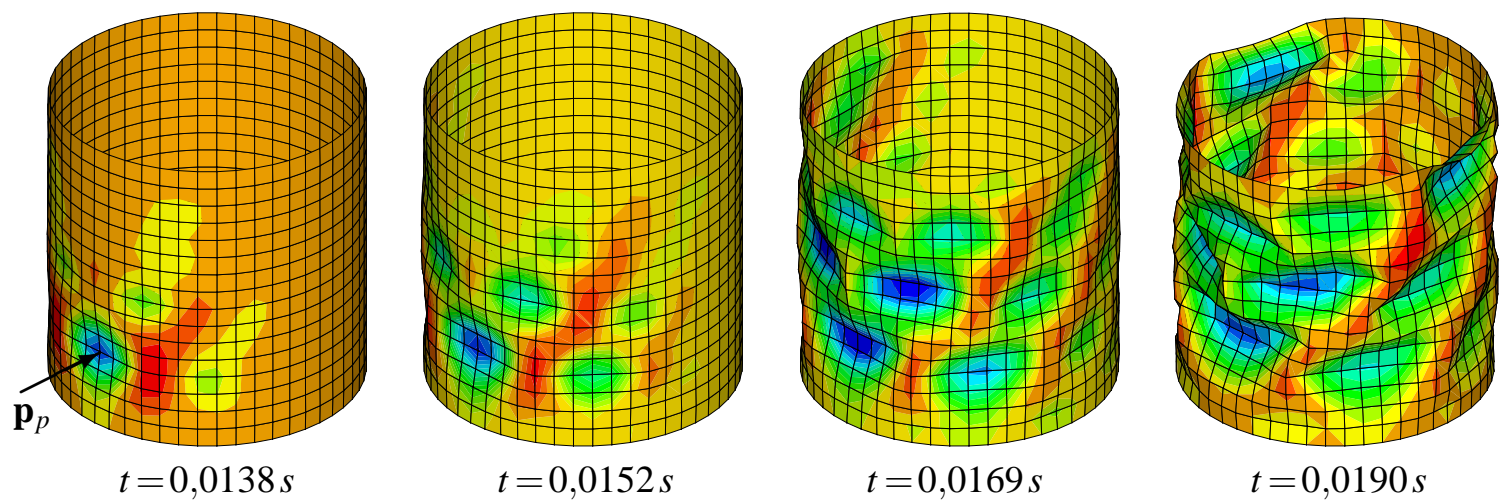

Bild 8.16: Störlast und Beulvorgang der Kreiszylinderschale

so beult die Kreiszylinderschale nicht rotationssymmetrisch, wobei signifikante nicht rotationssymmetrische Verformungen zuerst in der unteren Hälfte der Kreiszylinderschale festzustellen sind. Somit weisen die axialgedrückte Kreiszylinderschale und die in Abschnitt 8.1 analysierte Kugelschale unter Radialdruck bei einer grundbelastungsaffinen Störlast ein unterschiedliches Beulverhalten auf. Folgt die zeitliche Verteilung der Störlast einer Rampenfunktion, so ist die Stabilität des Systems bezüglich des dem neuen Lastniveau entsprechenden Grundzustandes zu bewerten. Dabei kann die Berechnung des Stabilitätsgrades mit der eingeprägten Energie $W_{0}$ nach Gl. (4.23) erfolgen, da der Bewegungszustand am Ende der Laststeigerung die Anfangsbedingungen der Systembewegung um den neuen Grundzustand beschreibt [32].

Die Untersuchung der Stabilität eines definierten Grundzustandes erlaubt folgenden Schluss: Die Störlasten, die nicht aus dem Störenergie-Konzept resultieren, müssen deutlich mehr Energie in das System einprägen, um Instabilität zu bewirken, als die Störlast gemäß Störenergie-Konzept. Insofern stellt diese Störlast die kinetische Grenzlast des Systems bezüglich des untersuchten Grundzustandes dar. Die Frage nach der Existenz einer realen Störlast, die der Störlast gemäß Störenergie-Konzept entspricht und somit auch Störverzerrungen aufweist, erscheint nicht berechtigt, da das Störenergie-Konzept Grenzbetrachtungen hinsichtlich der Stabilität eines Grundzustandes ermöglicht. Ferner kann die berechnete Störlast in ihrer Wirkung auf die Stabilität oftmals durch eine Einzellast ersetzt werden, da primär Angriffspunkt und Richtung der Störlast sowie die von dieser in das System eingeprägte Energie für die Bewertung der Stabilität relevant sind. Und gerade die räumliche Verteilung einer ungünstigen Störlast kann mit dem Störenergie-Konzept berechnet werden. Darüber hinaus ist aus den Bilder 8.1 und 8.14 eine Ähnlichkeit zwischen den Störlasten gemäß Störenergie-Konzept und einer Wirkung anfänglicher geometrischer Imperfektionen, nämlich die Entstehung auslenkender Radialkräfte [44], zu erkennen. 


\subsection{Zur Modifikation der Imperfektionsempfindlichkeit}

Sicherheitsbetrachtungen und die wirtschaftliche Bemessung von Schalentragwerken erfordern die Optimierung des Tragverhaltens hinsichtlich der Randstörungen, der dynamischen oder stabilitätstheoretischen Eigenschaften. Dabei können sich verschiedene Optimierungskriterien gegenseitig beeinflussen. So kann die Verringerung der Randstörungen eine Erhöhung der Imperfektionsempfindlichkeit bewirken [118].

Eine Möglichkeit zur Optimierung von Schalentragwerken resultiert nach Bewertung des Tragverhaltens mittels des Störenergie-Konzeptes aus dem Gedanken, die Imperfektionsempfindlichkeit für ein definiertes Lastniveau gezielt zu beeinflussen. Die Zielfunktion dieser lastniveauspezifischen Modifikation,

$$
f(x)=f\left(x, \mathbf{z}_{F}(x), \Delta \mathbf{z}(x)\right)=\Pi_{c r}(x)-\Pi_{c r}^{r e q}=0,
$$

fordert die Identifikation des kritischen Zustandes in Abhängigkeit von dem Entwurfsparameter $x$ und der vorgegebenen Störenergie $\Pi_{c r}^{r e q}$. Mittels Taylor-Entwicklung der Zielfunktion bezüglich des aktuellen Grundzustandes,

$$
f\left(x_{0}+\Delta x\right)=\Pi_{c r}\left(x_{0}\right)+\frac{d \Pi_{c r}\left(x_{0}\right)}{d x} \Delta x+\ldots-\Pi_{c r}^{r e q}=0,
$$

ist der erforderliche Zuwachs des Entwurfsparameters anzunähern. Die Ableitung der Störenergie nach dem Entwurfsparameter,

$$
\frac{d \Pi_{c r}\left(x_{0}\right)}{d x}=\frac{\partial \Pi_{c r}\left(x_{0}\right)}{\partial x}+\frac{\partial \Pi_{c r}\left(x_{0}\right)}{\partial \mathbf{z}_{F}} \frac{\partial \mathbf{z}_{F}}{\partial x}+\frac{\partial \Pi_{c r}\left(x_{0}\right)}{\partial \Delta \mathbf{z}} \frac{\partial \Delta \mathbf{z}}{\partial x},
$$

erfasst sowohl die Änderung des Grundzustandes als auch die der Richtung und des Abstandes zwischen Grundzustand und kritischem Zustand. Die Berechnung der Ableitung kann für den ersten Term analytisch erfolgen, erfordert aber für die anderen Terme eine numerische Differentiation. Zur Verringerung des Berechnungsaufwandes wird die Ableitung mit

$$
\frac{d \Pi_{c r}\left(x_{0}\right)}{d x} \approx \frac{\Pi_{c r}\left(x_{0}+\Delta \bar{x}\right)-\Pi_{c r}\left(x_{0}\right)}{\Delta \bar{x}}
$$

approximiert. Die Schrittweite $\Delta \bar{x}$ beeinflusst die Genauigkeit der ermittelten Ableitung und somit die Konvergenz der iterativ zu berechnenden Lösung. Für den Fall, dass die Zielfunktion hinsichtlich mehrerer Entwurfsparameter zu erfüllen ist, sind weitere Bedingungsgleichungen zur Ermittlung einer eindeutigen Lösung erforderlich. Die lastniveauspezifische Modifikation setzt für das jeweilige Lastniveau die Existenz eines kritischen Zustandes voraus. Richtung und Abstand zwischen Grundzustand und kritischem Zustand sollten während der Modifikation keine zu großen Änderungen erfahren. Je nach Entwurfsparameter ist die lastniveauspezifische Modifikation als Querschnitts- oder Faseroptimierung zu interpretieren [27]. Die mit der 
Modifikation einhergehende Änderung der Topologie der Energiefläche eines Systems ist indirekt an der Änderung des Last-Verformungs-Pfades sowie an der Entwicklung des Verlaufes der Grundenergie und der Störenergie erkennbar.

Für das Schalentragwerk nach Abschnitt 4.6.3 wird die lastniveauspezifische Modifikation aufgezeigt. Dabei soll die Empfindlichkeit des Systems gegenüber kinetischen Störlasten auf dem Lastniveau $P=250 \mathrm{~N}$ halbiert werden. Entwurfsparameter ist die Wanddicke $t$. Das Tragverhalten des Systems stellt Bild 8.17 heraus.
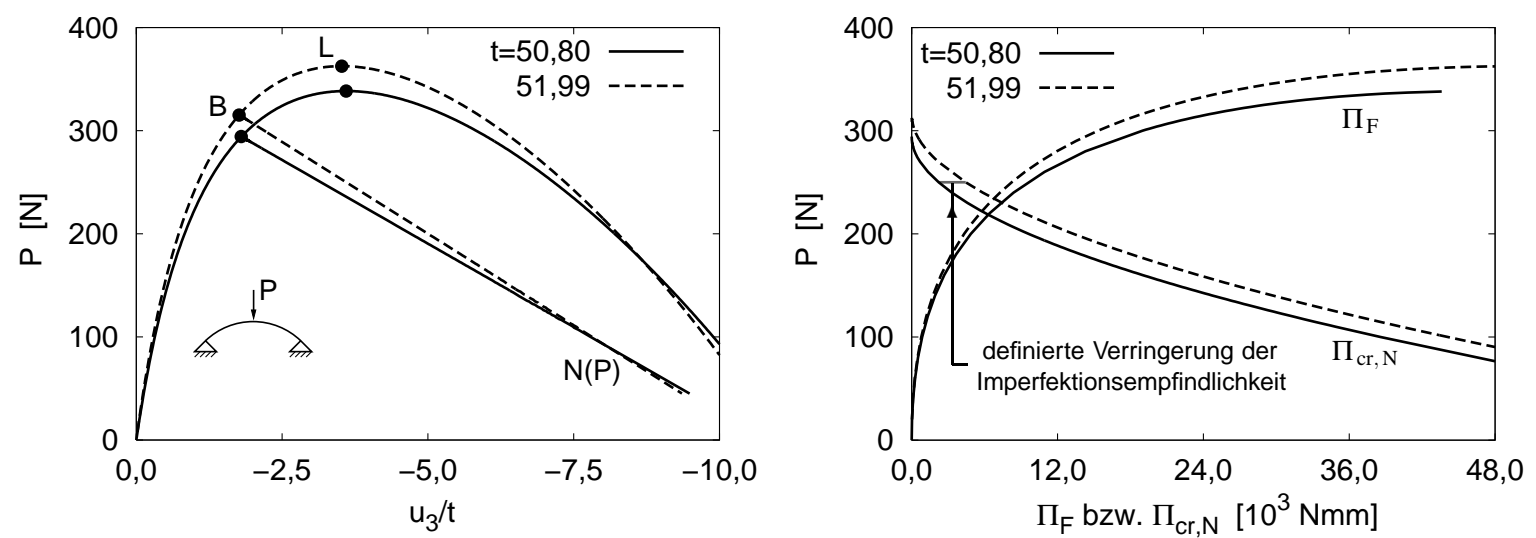

Bild 8.17: Last-Verformungs-Verhalten und Imperfektionsempfindlichkeit

Im Ausgangssystem beträgt die Wanddicke $50,8 \mathrm{~mm}$ und die Störenergie $\Pi_{c r, N}$ für das definierte Lastniveau $2,2 \cdot 10^{3} \mathrm{Nmm}$. Nach wenigen Iterationen entspricht die Störenergie dem geforderten Wert. Dabei erhöht sich die Wanddicke um 2,3\%. Diese geringfügige Erhöhung bewirkt die geforderte Halbierung der Imperfektionsempfindlichkeit. Die Steigung des Verlaufes der Störenergie wird nur unwesentlich von der Änderung der Wanddicke beeinflusst. Somit nimmt die erzielte prozentuale Verringerung der Imperfektionsempfindlichkeit von Grundzuständen unterhalb des definierten Lastniveaus ab. Die mit der Erhöhung der Wanddicke einhergehende Änderung des Abstandes zwischen Grundzustand und kritischem Zustand ist vom Lastniveau abhängig. Dennoch charakterisiert weiterhin ein unsymmetrischer Verformungsmode die Verformungen infolge Störlast. Ferner erhöht sich das Lastniveau des ersten singulären Punktes im primären Last-Verformungs-Pfad um 7,2\%. Die Grundenergie $\Pi_{F}$ wird indes unabhängig vom Lastniveau kleiner.

Die Ergebnisse können alternativ auch anhand einer systematischen Änderung der Wanddicke ermittelt werden. Der Vorteil der aufgezeigten Vorgehensweise zur lastniveauspezifischen Modifikation der Imperfektionsempfindlichkeit wird aber bei mehreren Entwurfsparametern und Systemen großer Dimension deutlich. 



\section{Zusammenfassung und Ausblick}

Das Störenergie-Konzept ermöglicht die Bewertung der Imperfektionsempfindlichkeit und der Grenzlasten von Schalentragwerken mit einer energetischen Größe, der Störenergie. Grundgedanke des Konzeptes ist die Identifikation des einem Grundzustand zugehörigen kritischen Zustandes. Die Differenz an Formänderungsenergie zwischen beiden Zuständen repräsentiert die Störenergie. Die Identifikation des kritischen Zustandes, der für die Stabilität des Grundzustandes maßgebend ist, lässt sich als Optimierungsproblem interpretieren. Dabei können anfängliche und nicht anfängliche Störungen berücksichtigt werden. Die Wirkung anfänglicher Störungen ist mit der störungsbedingten Änderung der Störenergie zu erfassen. Nicht anfängliche Störungen der Grundbelastung sind zum Erreichen eines kritischen Zustandes erforderlich. Entsprechende Störungen anderer Parameter der Modellgleichungen, wie dem Bettungsmodul und der Wanddicke, verdeutlichen die Ähnlichkeit des StörenergieKonzeptes mit der Störungsrechnung. Statische Grenzlasten sind mit dem Referenzwert der bezogenen Störenergie, kinetische Grenzlasten mit dem Stabilitätsgrad realistisch zu beschreiben. Eine Verringerung der Imperfektionsempfindlichkeit ist mittels der vorgestellten lastniveauspezifischen Modifikation möglich.

Die numerische Umsetzung des Störenergie-Konzeptes basiert auf dem inkrementellen elastischen Potential und der Methode der finiten Elemente. In der gemischten Formulierung werden die konstitutiven Gleichungen zur Berücksichtigung elastischplastischen Materialverhaltens und zur Beschreibung von Faserverbundwerkstoffen modifiziert. Das nichtlineare Eigenwertproblem, mit dem ein kritischer Zustand identifiziert wird, ist in der gemischten Formulierung und der Weggrößenformulierung mit der inversen Vektoriteration lösbar. Zur Integration der semidiskreten Bewegungsgleichung wird das Newmark-Verfahren verwendet.

Für Kreiszylinderschalen und Kugelschalen mit unterschiedlichen Belastungen, Geometrien, Randbedingungen und Materialparametern werden die Imperfektionsempfindlichkeit und die statische Grenzlast untersucht. Die Ergebnisse verdeutlichen, dass axialgedrückte Kreiszylinderschalen und Kugelschalen unter Radialdruck eine ähnlich große Imperfektionsempfindlichkeit aufweisen. Für diese Beulfälle zeigen die Grenzlasten gemäß Referenzwert der bezogenen Störenergie gute Übereinstimmung mit den Grenzlasten gemäß den ECCS-Recommendations, bei elastisch-plastischem 
Materialverhalten aber deutliche Differenzen zu denen nach DIN 18800. Die ermittelten Grenzlasten von Kegelschalen unter Meridianlast sind für verschiedene Öffnungswinkel den Grenzlasten nach DASt-Richtlinie 013 ähnlich. Ferner werden in den Regelwerken weniger intensiv diskutierte Beulfälle analysiert. Es ist aber nicht möglich, die Grenzlasten sämtlicher Beulfälle realistisch mit dem Referenzwert der bezogenen Störenergie zu beschreiben. Grund hierfür ist das Fehlen einer problemspezifische Normierung der Störenergie. Für Faserverbundwerkstoffe, die aus UDSchichten mit gleichen Materialparametern bestehen, wird eine geeignete Normierung angegeben. Die mit dieser Normierung ermittelten Grenzlasten sind indes noch anhand von Regelwerken und experimentellen Untersuchungen zu verifizieren.

Die Stabilität eines Schalentragwerkes gegenüber einer kinetischen Störlast ist von der Energie, die von der Störlast in das System eingeprägt wird, abhängig. Für eine Kugel- und eine Kreiszylinderschale wird der Einfluss der räumlichen und zeitlichen Verteilung der Störlast auf die Stabilität untersucht. Die Ergebnisse stellen die Bedeutung des Störenergie-Konzeptes sowohl für die Ermittlung ungünstiger Störlasten als auch für die Beschreibung der Widerstandsfähigkeit von Schalentragwerken heraus. Die Verringerung der Imperfektionsempfindlichkeit eines Grundzustandes um einen definierten Betrag wird für ein Schalentragwerk aufgezeigt.

Zukünftige Entwicklungen der Regelwerke sollten folgende Aspekte beachten: Die Verformungen infolge Störlast repräsentieren zumindest hinsichtlich Form und Position eine ungünstige anfängliche Imperfektion und sollten daher im Beulsicherheitsnachweis berücksichtigt werden. Der Grenzwert für die Tiefe von Vorbeulen könnte detaillierter in Abhängigkeit vom Beulfall festgelegt werden. Ferner wären Hinweise zur statischen Grenzlast axialgedrückter Kreiszylinderschalen, die am belasteten Rand frei sind, und zur Stabilität bei kurzzeitigen kinetischen Belastungen wünschenswert. 


\section{Summary and Outlook}

The perturbation energy concept enables to evaluate the imperfection sensitivity and the limit loads of shell structures by one energy value, the perturbation energy. Basic idea of the concept is the identification of the critical state belonging to a fundamental state. The difference of strain energy between both states represents the perturbation energy. The identification of the critical state, which is relevant for the stability of the fundamental state, is interpreted as an optimisation problem, whereas initial and noninitial perturbations may be considered. The effect of initial perturbations is measured by the associated change of the perturbation energy. Non-initial load perturbations are necessary to reach a critical state. Non-initial perturbations concerning other parameters of the governing equations, such as the bedding modulus and the wall thickness, emphasise the similarity between the perturbation energy concept and the perturbation theory. Static limit loads are described by the reference value of the normalised perturbation energy, kinetic limit loads by the degree of stability. The presented load-level-specific modification allows to reduce the imperfection sensitivity.

The numerical implementation of the perturbation energy concept is based on the incremental elastic potential and the finite element method. In the mixed formulation, the constitutive equations are modified to consider elasto-plastic material behaviour and to describe fibre-reinforced composites. The nonlinear eigenvalue problem which enables the identification of a critical state may be solved for the mixed formulation and the displacement formulation by the inverse vector iteration. The semi-discrete equation of motion is integrated applying the Newmark method.

For cylindrical and spherical shells, the imperfection sensitivity and the limit loads are analysed including different loadings, geometries, boundary conditions and material parameters. The results indicate that spherical shells under radial pressure are nearly as imperfection-sensitive as cylindrical shells subjected to axial load. For these buckling cases, limit loads according to the reference value of the normalised perturbation energy are in good agreement with those corresponding to the ECCS-Recommendations, but differ for elasto-plastic material behaviour significantly to those according to DIN 18800. The limit loads calculated for conical shells under meridional load are for different meridional angles similar to those specified by DASt-Richtlinie 013 . In addition, buckling cases less intensely discussed in the design rules are 
analysed. But it is not possible to specify realistic limit loads of all these buckling cases by the reference value of the normalised perturbation energy. This is to explain with the absence of a problem-specific scaling of the perturbation energy. For fibrereinforced composites consisting of unidirectional layers with equal material parameters, an appropriate scaling is deduced. The limit loads determined by this scaling are still to verify by design rules and data based on experimental investigations.

The stability of a shell structure against a kinetic perturbation load depends on the energy, which is induced into the system by this load. Therefore, the influence of the spatial and time distribution of the perturbation load on the stability of a spherical and a cylindrical shell is investigated. The results highlight the importance of the perturbation energy concept not only for the determination of unfavourable perturbation loads but also for the evaluation of the buckling resistance of shell structures. Finally, the reduction of the imperfection sensitivity of a fundamental state by a defined amount is exemplary performed for a shell structure.

Future developments of the design rules should consider the following aspects: The deformations due to the perturbation load represent at least an unfavourable initial imperfection with respect to shape and position. Thus, a verification of the buckling resistance should include such an imperfection. The tolerance value for the depth of initial dimples may be more accurately specified with regard to the buckling case. Furthermore, references for the evaluation of the static limit load of axially compressed cylindrical shells, which are free at the loaded boundary, and for the stability of shell structures in case of short-time kinetic loads would be appreciated. 


\section{Literatur}

[1] N. Akkas: Bifurcation and Snap-Through Phenomena in Asymmetric Dynamic Analysis of Shallow Spherical Shells. Comp. \& Struct. 6, 1976, 241-251.

[2] U. Albertin: Bemessungskonzepte für Stabilitätsfälle imperfektionssensitiver Schalenstrukturen. Dissertation, TU München, 2000.

[3] H. Altenbach, J. Altenbach \& R. Rikards: Einführung in die Mechanik der Laminatund Sandwichtragwerke. Deutscher Verlag der Grundstoffindustrie, Stuttgart, 1996.

[4] J. Altenbach \& H. Altenbach: Einführung in die Kontinuumsmechanik. Teubner, Stuttgart, 1994.

[5] U. Andelfinger: Untersuchungen zur Zuverlässigkeit hybrid-gemischter Finiter Elemente für Flächentragwerke. Dissertation, Universität Stuttgart, 1991.

[6] E. Anderson, Z. Bai, C. Bischof, S. Blackford, J. Demmel, J. Dongarra, J. Du Croz, A. Greenbaum, S. Hammarling, A. McKenney \& D. Sorensen: LAPACK Users' Guide. SIAM, Philadelphia, 1999.

[7] J. Arbocz \& H. Abramovich: The Initial Imperfection Data Bank at Delft University of Technology. Report LR-290, Delft University of Technology, 1979.

[8] Y. Başar \& W. B. Krätzig: Mechanik der Flächentragwerke. Vieweg, Braunschweig, 1985.

[9] Y. Başar \& D. Weichert: Nonlinear Continuum Mechanics of Solids. Springer, Berlin, 2000.

[10] Z. P. Bažant \& L. Cedolin: Stability of Structures. Oxford University Press, New York, 1991.

[11] P. G. Bergan, G. Horrigmoe, B. Krakeland \& T. H. Søreide: Solution Techniques for Non-Linear Finite Element Problems. Int. J. Num. Meth. Eng. 12, 1978, 1677-1696.

[12] P. Bernbeck: Die Anwendung direkter Zeitintegrationsverfahren auf Probleme der Strukturdynamik. Dissertation, TH Darmstadt, 1996.

[13] M. Bischoff: Theorie und Numerik einer dreidimensionalen Schalentheorie. Dissertation, Universität Stuttgart, 1999.

[14] J. Blachut \& G. D. Galletly: Buckling Strength of Imperfect Steel Hemispheres. ThinWalled Structures 23, 1995, 1-20. 
[15] K.-U. Bletzinger, M. Bischoff \& E. Ramm: A Unified Approach for Shear-LockingFree Triangular and Rectangular Shell Finite Elements. Comp. \& Struct. 75, 2000, 321-334.

[16] P. Bochev: A Discourse on Saddle-Point Problems, their Regularization, Stabilization and Finite Element Solution. Präsentation, Sandia National Laboratories, 2003.

[17] W. W. Bolotin: Kinetische Stabilität elastischer Systeme. VEB Deutscher Verlag der Wissenschaften, Berlin, 1961.

[18] F. W. Bornscheuer, L. Häfner \& E. Ramm: Zur Stabilität eines Kreiszylinders mit einer Rundschweißnaht unter Axialbelastung. Der Stahlbau 52, 1983, 313-318.

[19] D. Braess: Finite Elemente. Springer, Berlin, 2003.

[20] B. Brendel: Zur geometrisch nichtlinearen Elastostabilität. Dissertation, Universität Stuttgart, 1979.

[21] F. Brezzi \& M. Fortin: Mixed and Hybrid Finite Element Methods. Springer, New York, 1991.

[22] N. Büchter: Zusammenführung von Degenerationskonzept und Schalentheorie bei endlichen Rotationen. Dissertation, Universität Stuttgart, 1992.

[23] B. Budiansky: Theory of Buckling and Post-Buckling of Elastic Structures. Adv. Appl. Mech. 14, 1974, 2-63.

[24] B. Budiansky \& R. S. Roth: Axisymmetric Dynamic Buckling of Clamped Shallow Spherical Shells. NASA TND-1510 1962, 597-609.

[25] A. Burmeister: Dynamische Stabilität nach der Methode der finiten Elemente mit Anwendungen auf Kugelschalen. Dissertation, Universität Stuttgart, 1987.

[26] J. G. A. Croll \& R. C. Batista: Explicit Lower Bounds for the Buckling of Axially Loaded Cylinders. Int. J. Mech. Sci. 23, 1981, 331-343.

[27] F. Daoud: Formoptimierung von Freiformschalen - Mathematische Algorithmen und Filtertechniken. Dissertation, TU München, 2005.

[28] DASt-Richtlinie 013: Beulsicherheitsnachweise für Schalen. Deutscher Ausschuss für Stahlbau, Köln, 1980 (zurückgezogen 1992).

[29] DASt-Richtlinie 017: Beulsicherheitsnachweise für Schalen - spezielle Fälle. Deutscher Ausschuss für Stahlbau, Köln, 1992.

[30] M. Deml: Ein Finite-Element-Konzept zur Traglastanalyse imperfektionssensitiver Schalenstrukturen. Dissertation, TU München, 1997.

[31] DIN 18800-4: Stahlbauten, Stabilitätsfälle, Schalenbeulen. Deutsches Institut für Normung, Berlin, 1990.

[32] D. Dinkler: Eine Methode zum Nachweis der Beulsicherheit dünner Flächentragwerke bei zeitabhängigen Einwirkungen. ISD-Bericht Nr. 89/2, Universität Stuttgart, 1989. 
[33] D. Dinkler \& B. Kröplin: Stability of Dynamically Loaded Structures. In: W. B. Krätzig \& E. Oñate, Herausgeber, Computational Mechanics of Nonlinear Response of Shells. Springer, Berlin, 1990, 183-192.

[34] D. Dinkler \& U. Schäfer: Limit Loads for Cylindrical Shells under Combined Actions. In: Proc. Int. Conference on Carrying Capacity of Steel Shell Structures, Brno, 1997, 112-118.

[35] L. H. Donnell: A New Theory for the Buckling of Thin Cylinders under Axial Compression and Bending. ASME 56, 1934, 795-806.

[36] H. Duddeck, B. Kröplin, D. Dinkler, J. Hillmann \& W. Wagenhuber: Berechnung des nichtlinearen Tragverhaltens dünner Schalen im Vor- und Nachbeulbereich. In: E. Stein, Herausgeber, Nichtlineare Berechnungen im Konstruktiven Ingenieurbau. Springer, Berlin, 1989, 232-255.

[37] H. E. Düsing: Stabilität längsversteifter stählerner Kreiszylinderschalen unter zentrischem Axialdruck. Dissertation, Universität GH Essen, 1994.

[38] ECCS: European Recommendations - Buckling of Steel Shells. Brüssel, 1980.

[39] U. Eckstein, W. B. Krätzig \& U. Wittek: Wirklichkeitsnahe Grenzbeullasten von Rotationsschalen unterschiedlicher Gauss'scher Flächenkrümmung. Mitteilung Nr. 79-5, Institut für Konstruktiven Ingenieurbau, Ruhr-Universität Bochum, 1979.

[40] H. Eggers \& B. Kröplin: Yielding of Plates with Hardening and Large Deformations. Int. J. Num. Meth. Eng. 12, 1978, 739-750.

[41] I. Elishakoff: How to Introduce the Imperfection-Sensitivity Concept into Design. In: J. M. T. Thompson \& G. W. Hunt, Herausgeber, Collapse: The Buckling of Structures in Theory and Practice. Cambridge University Press, Cambridge, 1983, 345-357.

[42] C. Eller \& W. B. Krätzig: Numerische Stabilitätsanalyse linear und nichtlinear, deformierbarer, parametererregter Schalentragwerke. Ing.-Archiv 59, 1989, 345-356.

[43] R. Ender: Das Stabilitätsverhalten der axial gedrückten Kegelstumpfschale. Dissertation, Universität Hannover, 1981.

[44] M. Eßlinger: Eine Erklärung des Beulmechanismus von dünnwandigen Kreiszylinderschalen. Der Stahlbau 36, 1967, 366-371.

[45] M. Eßlinger \& B. Geier: Gerechnete Nachbeullasten als untere Grenze der experimentellen axialen Beullasten von Kreiszylindern. Der Stahlbau 41, 1972, 353-360.

[46] Eurocode 3: Design of Steel Structures - Part 1-6: Strength and Stability of Shell Structures - Draft Stage 49. CEN, Brüssel, 2005.

[47] E. Ewert, K. Schweizerhof \& P. Vielsack: Measures to Judge the Sensitivity of ThinWalled Shells Concerning Stability under Different Loading Conditions. Comp. Mech. 37, 2006, 507-522.

[48] J. R. Fitch \& B. Budiansky: Buckling and Postbuckling Behavior of Spherical Caps under Axisymmetric Load. AIAA J. 8, 1970, 686-693. 
[49] D. P. Flanagan \& T. Belytschko: A Uniform Strain Hexahedron and Quadrilateral with Orthogonal Hourglass Control. Int. J. Num. Meth. Eng. 17, 1981, 679-706.

[50] W. Flügge: Die Stabilität der Kreiszylinderschale. Ing.-Archiv 3, 1932, 463-506.

[51] H. Fritz \& U. Wittek: Zur Stabilität von Flächentragwerken. Mitteilung Nr. 74-6, Institut für Konstruktiven Ingenieurbau, Ruhr-Universität Bochum, 1974.

[52] R. E. Fulton \& F. W. Barton: Dynamic Buckling of Shallow Arches. J. Eng. Mech. Div. EM3, 1971, 865-877.

[53] G. D. Galletly: Torispherical Shells - A Caution to Designers. J. Eng. Industry 81, $1959,51-66$.

[54] G. D. Galletly, J. Kruzelecki, D. G. Moffat \& B. Warrington: Buckling of Shallow Torispherical Domes Subjected to External Pressure - A Comparison of Experiment, Theory, and Design Codes. J. Strain Analysis 22, 1987, 163-175.

[55] N. Gebbeken: Zur Untersuchung des linearen Tragverhaltens von Faserverbundkonstruktionen mittels numerischer Methoden. Habilitationsschrift, Universität der Bundeswehr München, 1996.

[56] M. Gee: Effiziente Lösungsstrategien in der nichtlinearen Schalenmechanik. Dissertation, Universität Stuttgart, 2004.

[57] B. Geier, H. Klein \& R. Zimmermann: Buckling Tests with Axially Compressed Unstiffened Cylindrical Shells Made from CFRP. In: J. F. Jullien, Herausgeber, Buckling of Shell Structures, on Land, in the Sea and in the Air. Elsevier, Essex, 1991, 498-507.

[58] D. Gerdes: Strukturoptimierung unter Anwendung der Optimalitätskriterien auf diskretisierte Tragwerke bei besonderer Berücksichtigung der Stabilität. Dissertation, Universität GH Essen, 1995.

[59] K. Giesen: Naturzug-Kühltürme. Vortragsveröffentlichungen, Haus der Technik e.V., Essen, 1968.

[60] K. Girkmann: Flächentragwerke. Springer, Wien, 1959.

[61] Y. Goldfeld, K. Vervenne, J. Arbocz \& F. van Keulen: Multi-Fidelity Optimization of Laminated Conical Shells for Buckling. Struct. Multidisc. Optim. 30, 2005, 128-141.

[62] W. Guggenberger: Buckling of Cylindrical Shells under Local Axial Loads. In: J. F. Jullien, Herausgeber, Buckling of Shell Structures, on Land, in the Sea and in the Air. Elsevier, Essex, 1991, 323-333.

[63] W. Hackbusch: Theorie und Numerik elliptischer Differentialgleichungen. Teubner, Stuttgart, 1996.

[64] R. Harbord: Berechnung von Schalen mit endlichen Verschiebungen - Gemischte finite Elemente. Dissertation, TU Braunschweig, 1972.

[65] R. Harbord \& A. Brenner: Schalenelemente in gemischt-hybrider Darstellung. In: W. Wunderlich, Herausgeber, Gemischte Finite Elemente. Institut für Konstruktiven Ingenieurbau, Ruhr-Universität Bochum, 1985. 
[66] R. Harbord, B. Kröplin \& R. Schröder: Schalenelemente in gemischter Darstellung: Theorie - Kritik - Beispiele. Ing.-Archiv 47, 1978, 207-222.

[67] F. Hartmann: The Discrete Babuška-Brezzi Condition. Ing.-Archiv 56, 1986, 221-228.

[68] C. Haußer: Effiziente Dreieckselemente für Flächentragwerke. Dissertation, Universität Stuttgart, 1996.

[69] A. Heinen, H.-M. Hennenberg \& O. Fischer: Kinetische Stabilität elastischer Schalentragwerke. Archive Appl. Mech. 64, 1994, 457-471.

[70] E. Hellinger: Die allgemeinen Ansätze der Mechanik der Kontinua. In: F. Klein \& C. Müller, Herausgeber, Enzyklopädie der Mathematischen Wissenschaften, Band 44. Teubner, Leipzig, 1914, 601-694.

[71] P. Helnwein: Zur initialen Abschätzbarkeit von Stabilitätsgrenzen auf nichtlinearen Last-Verschiebungspfaden elastischer Strukturen mittels der Methode der Finiten Elemente. Dissertation, TU Wien, 1996.

[72] K.-H. Herber: Vorschlag von Berechnungsgrundlagen für Beul- und Traglasten von Schalen. Der Stahlbau 35, 1966, 142-151.

[73] J. Hillmann: Grenzlasten und Tragverhalten axial gestauchter Kreiszylinderschalen im Vor- und Nachbeulbereich. Dissertation, TU Braunschweig, 1985.

[74] N. J. Hoff \& V. G. Bruce: Dynamic Analysis of the Buckling of Laterally Loaded Flat Arches. J. Math. Phys. 32, 1954, 276-288.

[75] H.-C. Hu: On Some Variational Methods on the Theory of Elasticity and the Theory of Plasticity. Scientia Sinica 4, 1955, 33-54.

[76] N. C. Huang: Unsymmetrical Buckling of Thin Shallow Spherical Shells. J. Appl. Mech. 31, 1964, 447-457.

[77] T. J. R. Hughes: The Finite Element Method. Prentice-Hall, Englewood Cliffs, 1987.

[78] T. J. R. Hughes \& T. E. Tezduyar: Finite Elements Based upon Mindlin Plate Theory with Particular Reference to the Four-Node Isoparametric Element. J. Appl. Mech. 48, 1981, 587-596.

[79] C. Hühne: Robuster Entwurf beulgefährdeter, unversteifter Kreiszylinderschalen aus Faserverbundwerkstoff. Dissertation, TU Braunschweig, 2006.

[80] C. Hühne, R. Rolfes \& J. Teßmer: Robust Design of Composite Shells: Simulation and Validation. In: B. H. V. Topping, G. Montero \& R. Montenegro, Herausgeber, Innovation in Engineering Computational Technology. Saxe Coburg Publications, Stirlingshire, 2006, 425-444.

[81] J. W. Hutchinson, R. C. Tennyson \& D. B. Muggeridge: Effect of a Local Axisymmetric Imperfection on the Buckling Behavior of a Circular Cylindrical Shell under Axial Compression. AIAA J. 9, 1971, 48-52.

[82] R. K. Jürke, W. B. Krätzig \& U. Wittek: Stabilitätsverhalten axialbelasteter Kreiszylinderschalen mit Regelimperfektionen nach DASt 013. Mitteilung Nr. 83-4, Institut für Konstruktiven Ingenieurbau, Ruhr-Universität Bochum, 1983. 
[83] R. Kao \& N. Perrone: Dynamic Buckling of Axisymmetric Spherical Caps with Initial Imperfections. Comp. \& Struct. 9, 1978, 463-473.

[84] M. Kleiber, H. Antúnez, T. D. Hien \& P. Kowalczyk: Parameter Sensitivity in Nonlinear Mechanics. Wiley \& Sons, Chichester, 1997.

[85] K. Klöppel \& O. Jungbluth: Beitrag zum Durchschlagproblem dünnwandiger Kugelschalen. Der Stahlbau 22, 1953, 121-130.

[86] K. Knebel: Stabilität von Stahlzylindern mit unilateralen Randbedingungen bei statischen und dynamischen Beanspruchungen. Dissertation, Universität Karlsruhe, 1997.

[87] O. Knoke: Beulwiderstände zusammengesetzter Zylinder-Kegel-Schalen. Dissertation, TU Braunschweig, 2003.

[88] W. T. Koiter: On the Stability of Elastic Equilibrium, Übersetzung von „Over de Stabiliteit van het elastisch Evenwicht". Dissertation, Delft, 1945.

[89] W. T. Koiter: On the Thermodynamic Background of Elastic Stability Theory. TU Delft, 1968.

[90] L. Kollár: Buckling of Complete Spherical Shells and Spherical Caps. In: E. Ramm, Herausgeber, Buckling of Shells. Springer, Berlin, 1982, 401-425.

[91] F. Koschnick, M. Bischoff \& K.-U. Bletzinger: Avoiding Membrane Locking with the DSG Method. In: Proc. of WCCM V, Wien, 2002.

[92] W. B. Krätzig, Y. Başar \& P. Nawrotzki: Dynamic Structural Instabilities. In: W. B. Krätzig \& H.-J. Niemann, Herausgeber, Dynamics of Civil Engineering Structures. Balkema, Rotterdam, 1996, 377-449.

[93] H. Krause: Flattern flacher Schalen bei Überschallanströmung. Dissertation, Universität Stuttgart, 1998.

[94] E. Kreuzer: Numerische Untersuchung nichtlinearer dynamischer Systeme. Springer, Berlin, 1987.

[95] B. Kröplin: Quasi viskose Berechnung von nichtlinearen Stabilitätsproblemen. Habilitationsschrift, TU Braunschweig, 1982.

[96] B. Kröplin, D. Dinkler \& J. Hillmann: An Energy Perturbation Applied to Nonlinear Structural Analysis. Comp. Meth. Appl. Mech. Eng. 52, 1985, 885-897.

[97] R. Krysik: Stabilität stählerner Kegelstumpf- und Kreiszylinderschalen unter Axialund Innendruck. Dissertation, Universität GH Essen, 1994.

[98] I. Kubor: Nonlinear Deformation and Buckling of Shells Weakened by Holes of Complex Curvilinear Configurations. In: J. M. T. Thompson \& G. W. Hunt, Herausgeber, Collapse: The Buckling of Structures in Theory and Practice. Cambridge University Press, Cambridge, 1983, 395-408.

[99] D. Kuhl: Stabile Zeitintegrationsalgorithmen in der nichtlinearen Elastodynamik dünnwandiger Tragwerke. Dissertation, Universität Stuttgart, 1996. 
[100] C. Lanczos: The Variational Principles of Mechanics. University of Toronto Press, Toronto, 1949.

[101] H. Leipholz: Stabilitätstheorie. Teubner, Stuttgart, 1968.

[102] H. Leipholz: Stabilität elastischer Systeme. Braun, Karlsruhe, 1980.

[103] J. Lindner, J. Scheer \& H. Schmidt: Stahlbauten - Erläuterungen zu DIN 18800 Teil 1 bis Teil 4. Beuth und Ernst \& Sohn, 1993.

[104] J. G. Malkin: Theorie der Stabilität einer Bewegung. Oldenbourg, München, 1959.

[105] H. A. Mang, C. Schranz \& P. Mackenzie-Helnwein: Conversion from ImperfectionSensitive into Imperfection-Insensitive Elastic Structures I: Theory. Comp. Meth. Appl. Mech. Eng. 195, 2005, 1422-1457.

[106] J. Marcinowski: Stability of a Spherical Cap Loaded by External Pressure. In: Proc. 8th SSTA Conference, Jurata, 2005, 241-244.

[107] G. Markus: Theorie und Berechnung rotationssymmetrischer Bauwerke. Werner, Düsseldorf, 1978.

[108] K. M. Mushtari \& A. V. Sachenkov: Stability of Cylindrical and Conical Shells of Circular Cross Section, with Simultaneous Action of Axial Compression and External Normal Pressure. NACA TM 1433, 1965.

[109] NASA SP-8007: Buckling of Thin-Walled Circular Cylinders. Washington, 1968.

[110] NASA SP-8019: Buckling of Thin-Walled Truncated Cones. Washington, 1968.

[111] P. Nawrotzki: Ein Beitrag zur numerischen Stabilitätsanalyse stationärer und instationärer Strukturantworten. Dissertation, Ruhr-Universität Bochum, 1994.

[112] P. Nawrotzki, W. B. Krätzig \& U. Montag: Dynamic Instability Analysis of Elastic and Inelastic Shells. Comp. Mech. 21, 1998, 48-59.

[113] H. Obrecht, B. Rosenthal, P. Fuchs, S. Lange \& C. Marusczyk: Buckling, Postbuckling and Imperfection-Sensitivity: Old Questions and some New Answers. Comp. Mech. 37, 2006, 498-506.

[114] C. Petersen: Dynamik der Baukonstruktionen. Vieweg, Braunschweig, 1996.

[115] A. Pflüger: Stabilität dünner Kegelschalen. Ing.-Archiv VIII, 1937, 151-172.

[116] A. Pflüger: Stabilitätsprobleme der Elastostatik. Springer, Berlin, 1964.

[117] E. Reissner: On a Variational Theorem in Elasticity. J. Math. and Physics 29, 1950, 90-95.

[118] R. Reitinger: Stabilität und Optimierung imperfektionsempfindlicher Tragwerke. Dissertation, Universität Stuttgart, 1994.

[119] H. J. Rensch: Elastoplastisches Beulen und Imperfektionsempfindlichkeit torisphärischer Schalen. Dissertation, Ruhr-Universität Bochum, 1982. 
[120] E. Riks: The Application of Newton's Method to the Problem of Elastic Stability. J. Appl. Mech. 39, 1972, 1060-1066.

[121] T. Rottner: Sensitivitätsanalyse stabiler Gleichgewichtslagen dünnwandiger Strukturen unter Verwendung von Lösungsverfahren für Parallelrechner. Dissertation, Universität Karlsruhe, 2000.

[122] L. Å. Samuelson \& S. Eggwertz: Shell Stability Handbook. Elsevier, Essex, 1992.

[123] P. T. Saunders: Katastrophentheorie - Eine Einführung für Naturwissenschaftler. Vieweg, Braunschweig, 1986.

[124] U. Schäfer: Grenzlasten von Kreiszylinderschalen bei elastisch-plastischem Materialverhalten. Dissertation, Universität Stuttgart, 1997.

[125] K. Schiffner: Untersuchung des Stabilitätsverhaltens dünnwandiger Kegelschalen unter axialsymmetrischer Belastung mittels einer nichtlinearen Schalentheorie. WGLRJahrbuch, 1965, 448-453.

[126] R. Schlebusch: Theorie und Numerik einer oberflächenorientierten Schalenformulierung. Dissertation, TU Dresden, 2005.

[127] W. Schneider: Stimulating Equivalent Geometric Imperfections for the Numerical Buckling Strength Verification of Axially Compressed Cylindrical Steel Shells. Comp. Mech. 37, 2006, 530-536.

[128] W. Schneider \& M. Gettel: Equivalent Geometric Imperfections for Steel Shell Structures Subject to Combined Loading. In: Proc. III ECCM Conference, Lissabon, 2006.

[129] W. Schnell: Die dünnwandige Kegelschale unter Axial- und Innendruck. Zeitschrift für Flugwissenschaften 10, 1962, 154-160 und 314-321.

[130] H. R. Schwarz: Methode der finiten Elemente. Teubner, Stuttgart, 1984.

[131] K. Schweizerhof: Nichtlineare Berechnung von Tragwerken unter verformungsabhängiger Belastung mit finiten Elementen. Dissertation, Universität Stuttgart, 1982.

[132] P. Seide: Axisymmetrical Buckling of Circular Cones under Axial Compression. J. Appl. Mech. 23, 1956, 625-628.

[133] SFB 151: Tragverhalten und Tragfähigkeit von Baukonstruktionen unter dynamischen Einwirkungen. Sonderforschungsbereich 151, Ruhr-Universität Bochum, 1982-1994.

[134] R. T. Shield \& A. E. Green: On Certain Methods in the Stability Theory of Continuous Systems. Archive Rat. Mech. Anal. 12, 1963, 354-360.

[135] G. J. Simitses: Dynamic Stability of Suddenly Loaded Structures. Springer, New York, 1990.

[136] J. C. Simo \& M. S. Rifai: A Class of Mixed Assumed Strain Methods and the Method of Incompatible Modes. Int. J. Num. Meth. Eng. 29, 1990, 1595-1638.

[137] J. C. Simo \& N. Tarnow: A New Energy and Momentum Conserving Algorithm for the Non-Linear Dynamics of Shells. Int. J. Num. Meth. Eng. 37, 1994, 2527-2549. 
[138] G. L. G. Sleijpen, A. G. L. Booten, D. R. Fokkema \& H. A. van der Vorst: JacobiDavidson Type Methods for Generalized Eigenproblems and Polynomial Eigenproblems. BIT 36, 1996, 595-633.

[139] I. Spohr: Störenergie-Konzept für den elasto-plastischen Beulsicherheitsnachweis beliebig belasteter Zylinderschalen. Dissertation, TU Braunschweig, 1998.

[140] O. Steinhardt \& U. Schulz: Zum Beulverhalten von Kreiszylinderschalen. Schweizerische Bauzeitung 89, 1971, 1-14.

[141] P. Swadlo: Stabilität zusammengesetzter stählerner Kreiszylinder-Kegel-Schalen unter Axialdruck und Außendruck. Dissertation, Universität GH Essen, 2001.

[142] G. A. Thurston \& A. A. Holston: Buckling of Cylindrical Shell End Closures by Internal Pressure. NASA CR-540, 1966.

[143] S. Timoshenko: Einige Stabilitätsprobleme der Elastizitätstheorie. Z. Math. Physik $58,1910,337-385$.

[144] G. Tranel: Stabilitätsnachweis beliebiger Schalen mit dem Konzept der Störenergie. Dissertation, TU Braunschweig, 1994.

[145] T. v. Kármán \& H. S. Tsien: The Buckling of Spherical Shells by External Pressure. J. Aero. Sci. 7, 1939, 43-50.

[146] T. v. Kármán \& H. S. Tsien: The Buckling of Thin Cylindrical Shells under Axial Compression. J. Aero. Sci. 8, 1941, 303-312.

[147] A. van der Neut: De elastische Stabiliteit van den dunwandigen Bol. Dissertation, Delft, 1932.

[148] D. Vandepitte, J. Rathe, B. Verhegghe, R. Paridaens \& C. Verschaeve: Experimental Investigation of Buckling of Hydrostatically Loaded, Conical Shells and Practical Evaluation of the Buckling Load. In: E. Ramm, Herausgeber, Buckling of Shells. Springer, Berlin, 1982, 375-399.

[149] P. Vielsack, W. Sprenger \& T. Hesse: Sensitivität stabiler Gleichgewichtszustände elastischer Strukturen. Stahlbau 64, 1995, 321-325.

[150] H. Voss: An Arnoldi Method for Nonlinear Eigenvalue Problems. BIT 44, 2004, 387401.

[151] W. Wagenhuber: Imperfektionssensitivität und rechnerischer Nachweis der Beulsicherheit dünner Schalen. Dissertation, TU Braunschweig, 1989.

[152] W. Wagner: Zur Behandlung von Stabilitätsproblemen der Elastostatik mit der Methode der Finiten Elemente. Habilitationsschrift, Universität Hannover, 1991.

[153] L. R.-L. Wang: Discrepancy of Experimental Buckling Pressures of Spherical Shells. AIAA J. 5, 1967, 357-359.

[154] K. Washizu: On the Variational Principles of Elasticity and Plasticity. Technical Report 25-18, MIT Cambridge, 1955. 
[155] H. J. Weinitschke: On Asymmetric Buckling of Shallow Spherical Shells. J. Math. and Physics 44, 1965, 141-163.

[156] F. A. Weise: Untersuchungen über das Beul- und Nachbeulverhalten dünnwandiger rotationssymmetrischer Schalentragwerke unter Randlast mittels finiter Ringelemente. Dissertation, RWTH Aachen, 1975.

[157] G. A. Wempner: Discrete Approximations Related to Nonlinear Theories of Solids. Int. J. Solids Struct. 7, 1971, 1581-1599.

[158] M. Wessels: Das statische und dynamische Durchschlagproblem der imperfekten flachen Kugelschale bei elastischer rotationssymmetrischer Verformung. Dissertation, TU Hannover, 1977.

[159] K. Willner: Kontinuums- und Kontaktmechanik - Synthetische und analytische Darstellung. Springer, Berlin, 2003.

[160] T. A. Winterstetter \& H. Schmidt: Stability of Circular Cylindrical Shells under Combined Loading. Thin-Walled Struct. 40, 2002, 893-909.

[161] U. Wittek \& K. Grote: Abschätzungskonzepte zur Schalenbeulung. In: E. Stein, Herausgeber, Nichtlineare Berechnungen im Konstruktiven Ingenieurbau. Springer, Berlin, 1989, 276-299.

[162] W. Wunderlich, H. Obrecht \& F. Schnabel: Beulverhalten von Kugelschalen unter stetig veränderlichen Flächenlasten. Schlussbericht zum Forschungsvorhaben Nr. 16.65, Institut für Bautechnik, Berlin, Lehrstuhl für Statik, TU München, Institut für Konstruktiven Ingenieurbau, Ruhr-Universität Bochum, 1989.

[163] W. Wunderlich, F. Schnabel \& H. Obrecht: Tragfähigkeit zusammengesetzter Rotationsschalen im geometrisch und physikalisch nichtlinearen Bereich. Mitteilung Nr. 86-3, Institut für Konstruktiven Ingenieurbau, Ruhr-Universität Bochum, 1986.

[164] W. Zerna, Y. Başar, I. Mungan \& W. Tiemann: Beuluntersuchungen an hyperbolischen Rotationsschalen. Forschungsbericht Nr. 2439, Institut für Konstruktiven Ingenieurbau, Ruhr-Universität Bochum, 1974.

[165] W. Zerna \& I. Mungan: Buckling Stresses of Shells Having Negative Gaussian Curvature. In: E. Ramm, Herausgeber, Buckling of Shells. Springer, Berlin, 1982, 467-485.

[166] H. Ziegler: Die Stabilitätskriterien der Elastomechanik. Ing.-Archiv 20, 1952, 49-56.

[167] G. Zielke: Numerische Berechnung von benachbarten inversen Matrizen und linearen Gleichungssystemen. In: P. Schmitz \& C. Heinrich, Herausgeber, Schriften zur Datenverarbeitung, Band 2. Vieweg, Braunschweig, 1970.

[168] O. C. Zienkiewicz, R. L. Taylor \& J. M. Too: Reduced Integration Techniques in General Analysis of Plates and Shells. Int. J. Num. Meth. Eng. 3, 1971, 275-290.

[169] R. Zoelly: Über ein Knickungsproblem an der Kugelschale. Dissertation, TH Zürich, 1915. 


\section{Berichte aus dem Institut für Statik ab 1994}

$\begin{array}{lrl}\text { Nr. } & 94-78 & \begin{array}{l}\text { U. KOWALSKY: Mikrophysikalisch begründetes Werkstoffmodell zur } \\ \text { Berechnung thermomechanisch beanspruchter Konstruktionen (1994). }\end{array} \\ \text { Nr. } & 95-79 & \begin{array}{l}\text { N. OBERBECK: Instationärer Wärme-Feuchte-Schadstoff - Transport } \\ \text { in Beton, Theorie und Berechnung (1995). }\end{array} \\ \text { Nr. } & 95-80 & \begin{array}{l}\text { N. MELEKA: Nonlinear Analysis of Flat Slab Floors (1995). } \\ \text { Nr. }\end{array} \\ 97-81 & \begin{array}{l}\text { H. FLOREN: Strukturanalysen unterirdischer Hohlräume im Salzgebir- } \\ \text { ge mit hybriden Randelement-/Finite-Element-Methoden (1997). }\end{array}\end{array}$

Nr. 96-82 T. GARTUNG: Berechnung von Asphaltstraßen mit einem einheitlichen rheologischen Konzept einschließlich Schädigung (1996).

Nr. $\quad 97-83 \quad$ T. STREILEIN: Erfassung formativer Verfestigung in viskoplastischen Stoffmodellen (1997).

Nr. 97-84 B. GUERICKE: Geomechanische Untersuchungen zur Sicherheitsanalyse von Deponien im Salzgestein (1997).

Nr. $\quad$ 97-85 U. GLABISCH: Stoffmodell für Grenzzustände im Salzgestein zur Berechnung von Gebirgshohlräumen (1997).

Nr. $\quad 97-86$ E. ELARABI: Numerical Analysis of the Swelling Soil Effects on Structures (1997).

Nr. $\quad 98-87 \quad$ I. SPOHR: Störenergie-Konzept für den elasto-plastischen Beulsicherheitsnachweis beliebig belasteter Zylinderschalen (1998).

Nr. $\quad 99-88 \quad$ C.-X. HUANG: Berechnung von Temperaturspannungen und Rißbildungen infolge Hydratation in 3D-Betonkonstruktionen (1999).

Nr. 2000-89 H. PENSKY: Beschreibung von streuendem Materialverhalten und von Schädigung bei inelastischen Werkstoffen (2000).

Nr. 2000-90 A. STEFFENS: Modellierung von Karbonatisierung und Chloridbindung zur numerischen Analyse der Korrosionsgefährdung der Betonbewehrung (2000).

Nr. 2001-91 J.EBERS-ERNST: Modellierung des inelastischen Verformungsverhaltens von Siedlungsabfalldeponien (2001).

Nr. 2001-92 J. HANEL: Modell zur Analyse von gekoppelten Transport- und Stoffabbauprozessen in Deponien (2001).

Nr. 2002-93 B. WIEDEMANN: Modelle für vorgespannte Membranen mit Faltenbildung (2002).

Nr. 2002-94 R. TACKE: Feuchte- und Festigkeitsentwicklung hydratisierenden Betons - Modellierung und numerische Analyse (2002). 
Nr. 2002-95 E. WALHORN: Ein simultanes Berechnungsverfahren für Fluid-Struktur-Wechselwirkungen mit finiten Raum-Zeit-Elementen (2002).

Nr. 2003-96 $\quad$ B. HÜBNER: Simultane Analyse von Bauwerk-Wind-Wechselwirkungen (2003).

Nr. 2003-97 J. GEISTEFELDT: Stochastische Finite-Element-Methoden mit Anwendung auf aeroelastische Tragsysteme (2003).

Nr. 2003-98 O. KNOKE: Beulwiderstände zusammengesetzter Zylinder-KegelSchalen (2003).

Nr. 2005-99 A. KÖLKE: Modellierung und Diskretisierung bewegter Diskontinuitäten in randgekoppelten Mehrfeldsystemen (2005).

Nr. 2006-100 D. DINKLER (HERAUSGEBER): Institut für Statik - Lehre und Forschung, 1996 - 2006 (2006).

Nr. 2006-101 A. VEHRE: Ein Reduktionsverfahren für Fluid-Struktur-Wechselwirkungen mit finiten Raum-Zeit-Elementen (2006).

Nr. 2006-102 M. LÖHR: Analyse aeroelastischer Systeme mit Spektralen Stochastischen Finite-Element-Methoden (2006).

Nr. 2006-103 L. ASCHENBRENNER: Mehrkomponenten-Modell zur Beschreibung des Deformationsverhaltens von Asphalt (2006).

Nr. 2006-104 T. ZÜMENDORF: Ein gradientenabhängiges Modell für Schädigung bei viskoplastischem Materialverhalten (2006).

Nr. 2007-105 C. LEPPERT: Mehrphasenmodell für granulare Medien zur numerischen Untersuchung des Phasenübergangs bei der Entleerung von Silos (2007).

Nr. 2008-106 V. KRASE: Stability of Municipal Solid Waste Landfills (2008).

\section{Sonderdrucke}

Phänomenologische Modelle für Werkstoffe des Bauwesens.

Hermann Ahrens zum 60. Geburtstag (1998).

Herausgeber: D. Dinkler, U. Kowalsky.

Baustatik-Baupraxis 8.

Berichte der Fachtagung am 21. und 22. März 2002 in Braunschweig.

Herausgeber: D. Dinkler.

Institut für Statik, Technische Universität Braunschweig

Beethovenstraße 51, 38106 Braunschweig, Deutschland

Telefon: +49 (0)531/391-3668, Telefax: +49 (0)531/391-8116

E-Mail: statik@tu-bs.de, Homepage: http://www.statik.tu-braunschweig.de 